Heat Transfer Enhancement of Vapor Condensation Heat Exchanger

A Dissertation

Presented to the

Faculty of the Graduate School

at the University of Missouri-Columbia

In Partial Fulfillment

of the Requirements for the Degree

Doctor of Philosophy

by

Husam Rajab

Dr. Hongbin Ma, Dissertation Supervisor

May 2017 
The undersigned, appointed by the dean of the Graduate School, have examined the dissertation entitled

\section{Heat Transfer Enhancement of Vapor Condensation Heat Exchanger}

presented by Husam Rajab,

a candidate for the degree of Doctor of Philosophy in Mechanical Engineering, and hereby certify that, in their opinion, it is worthy of acceptance:

Professor Hongbin Ma

Professor Gary Solbrekken

Professor Yuwen Zhang

Professor Matt Maschmann

Professor Shubhra Gangopadhyay 


\section{ACKNOWLEGEMENT}

I would like to thank my Ph.D supervisor Dr. Hongbin Ma for his patient guidance and solid support of my research project and my degree program. Without his academic insight and encouragement, this dissertation would not have been possible. I would like to thank my dissertation committee Dr. Gary Solbrekken, Dr. Yuwen Zhang, Dr. Matt Maschmann and Dr. Shubhra Gangopadhyay for their time and efforts. Their broad knowledge and many stimulating discussions are invaluable and greatly appreciated. I would also like to thank all the students, professors and staff of Mechanical and Aerospace Engineering department for their support and help driving me to complete my Ph.D degree successfully.

Finally I would like to thank my family and my friends for their continuous support and encouragement to complete my research and final dissertation.

The supports of the MU Innovations Fund (IF) Center and the MU College of Engineering CNC Milling Machine Laboratory are gratefully acknowledged. 


\section{TABLE OF CONTENTS}

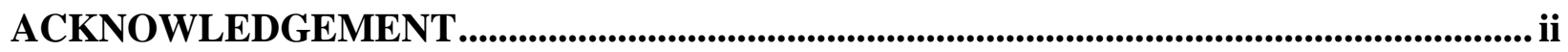

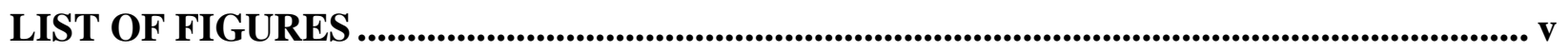

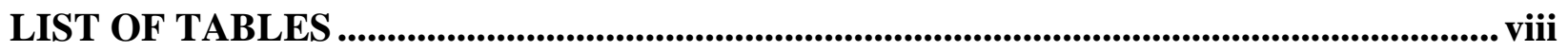

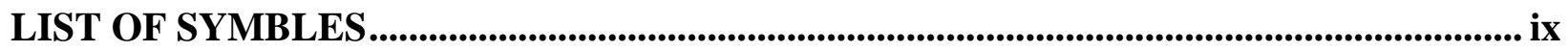

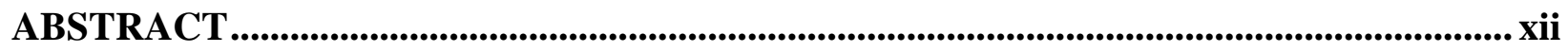

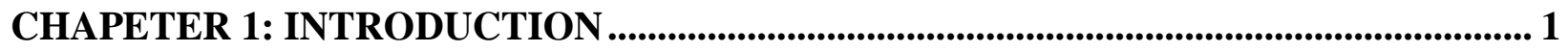

1.1. PRINCIPLES OF CONDENSATON AND EVAPORATION ............................... 1

1.2. COOLING TOWER MATERIALS SELECTION ............................................ 2

1.3. IDENTIFYING STEAM POWER SYSTEMS .................................................. 3

1.4. OBJECTIVE \& RESEARCH APPROACH ..................................................... 4

CHAPETER 2: HEAT TRANSFER ENHANCEMENT USING MINI/MICRO

ELLIPTICAL PIN-FIN HEAT SINKS WITH NANOFLUIDS....................................... 6

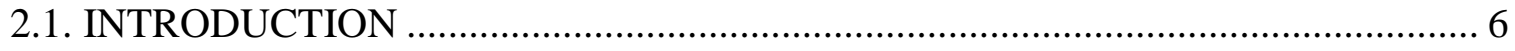

2.2. MATHEMATICAL MODEL AND GOVERNING EQUATIONS......................... 7

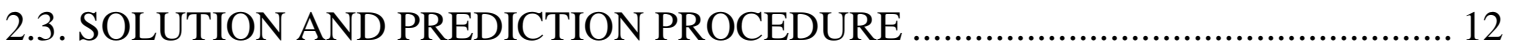

2.4. GRID INDEPENDENCY AND CODE VALIDATION ...................................... 13

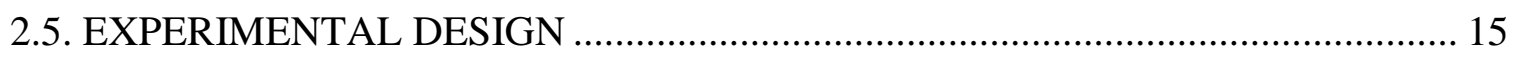

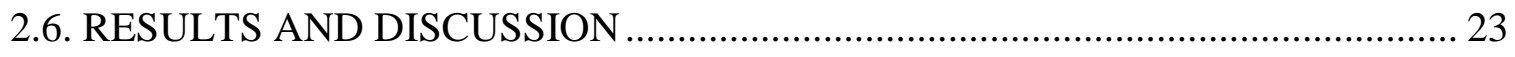

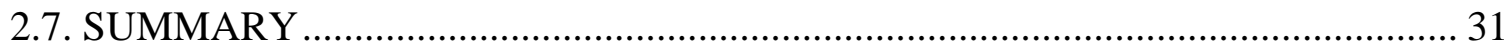

\section{CHAPTER 3:UTILIZATION OF THIN FILM EVAPORATION IN A TWO-PHASE}

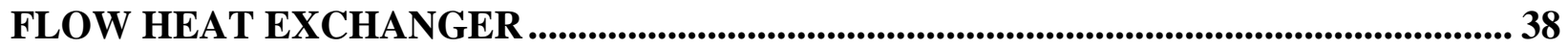

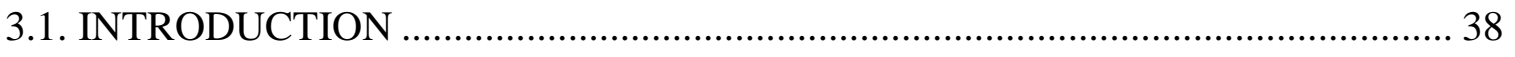

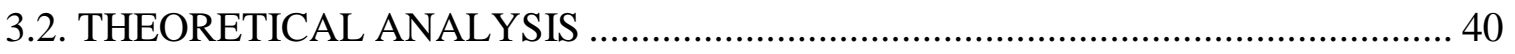




\section{CHAPTER 4: NON-DIMENSIONAL ANALYSIS OF TWO-PHASE FLOW AND} CONDENSATION HEAT TRANSFER IN POROUS MEDIUM ...................................... 56

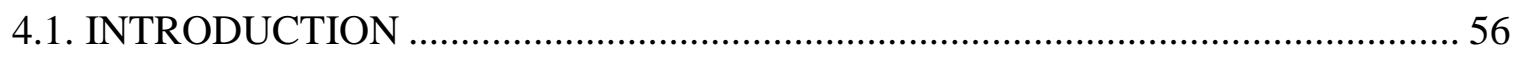

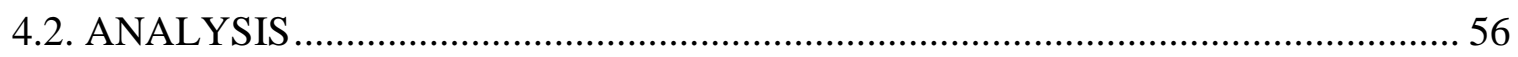

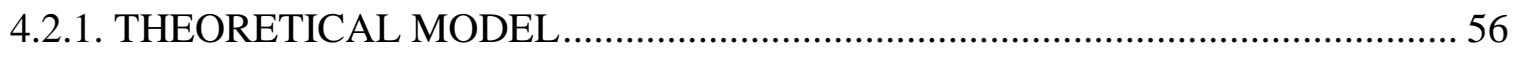

4.2.2. SOLUTION AND PREDICTION PROCEDURE ........................................ 72

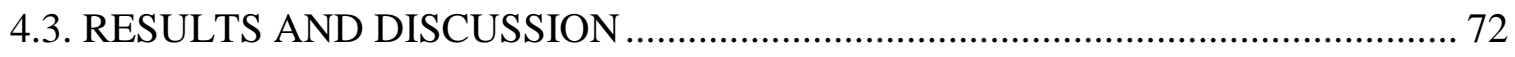

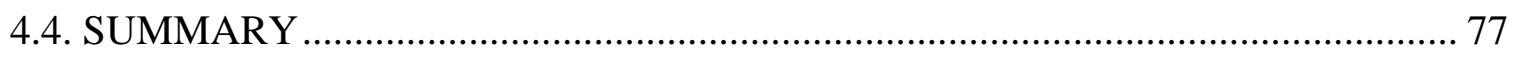

CHAPTER 5: VAPOR CONDENSATION HEAT TRANSFER IN POROUS MEDUM... 78

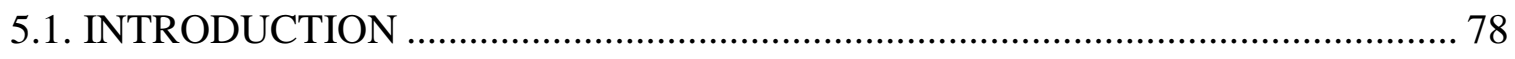

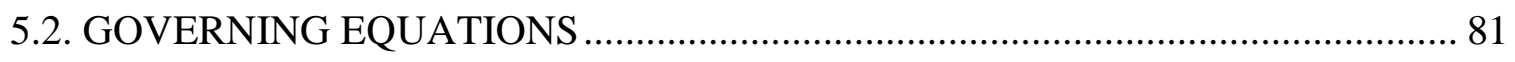

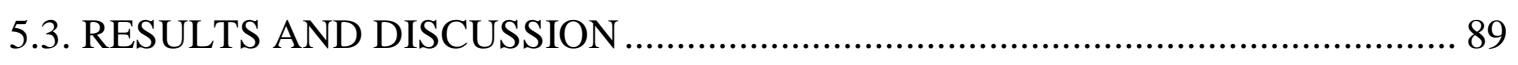

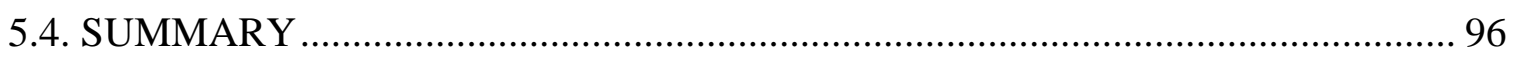

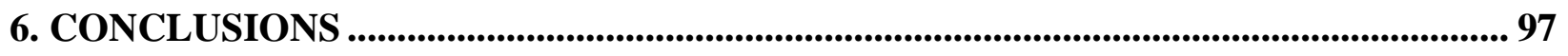

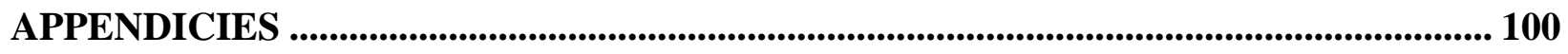

APPENDIX A: POROUS MEDIUM MATHEMATICAL DERIVATIONS ................. 99

APPENDIX B: DESIGN AND MANUFACTURING OF MPFHS …...................... 105

APPENDIX C: C1. HEAT SINK PROGRAMMING CODES ................................. 106

APPENDIX C: C2. POROUS MEDIUM PROGRAMMING CODES ….................... 107

APPENDIX C: C3. HEAT SINK EXPERIMENTAL DESIGN CALCULATIONS .... 117

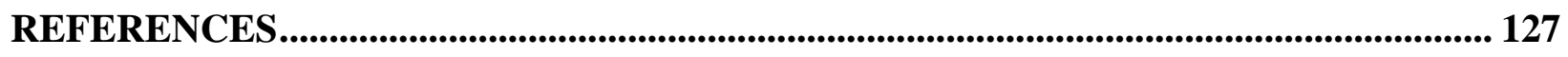

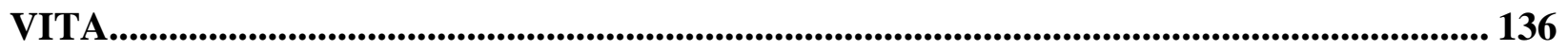




\section{LIST OA FIGURES}

FIG.1.1. VAPOR-TO-CONDENSATE HEAT EXCHANGER PROCESS................................... 1

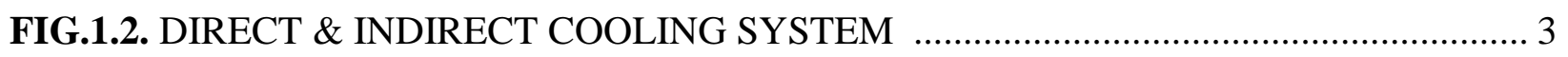

FIG.1.3. WATER FLOW IN ONCE-THROUGH COOLING ..................................................... 4

FIG.1.4. TYPICAL VAPOR CONDENSATION SYSTEM ………………………………….... 5

FIG.2.1. SCHEMATIC OF THE ELLIPTICAL PIN-FIN HEAT SINK. (LEFT: PINS

ARRANGED AT THE SAME ANGLE; RIGHT: PINS ARRANGED AT DIFFERENT

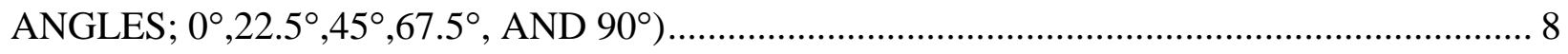

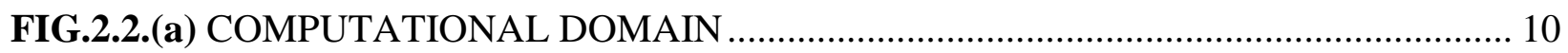

FIG.2.2.(b) NONUNIFORM COMPUTATIONAL GRID: (TOP): PINS HAVE SAME ORIENTATION ANGLES, AND (BOTTOM): PINS HAVE DIFFERENT ORIENTATION

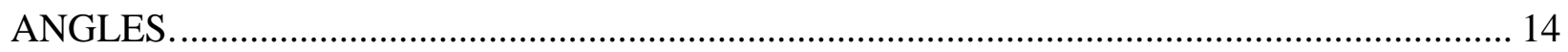

FIG.2.3. SCHEMATIC OF EXPERIMENTAL SETUP ……………………………............ 15

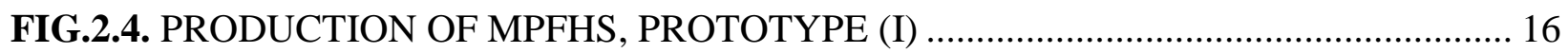

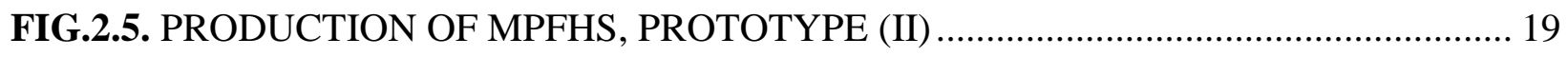

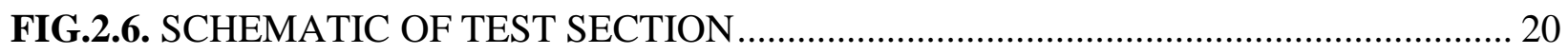

FIG.2.7.(a) VISUALIZATION OF THE FLOW PATTERN: TOP: CURRENT RESULTS,

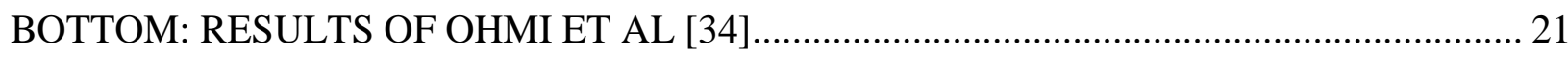

FIG.2.7.(b) A PHOTOGRAPH OF THE EXPERIMENT .............................................................. 22

FIG.2.8. FLUID AND SURFACE AXIAL TEMPERATURE VARIATIONS ........................... 22

FIG.2.9. HEAT TRANSFER COEFFICIENT ALONG THE LENGTH OF THE PIN FIN HEAT

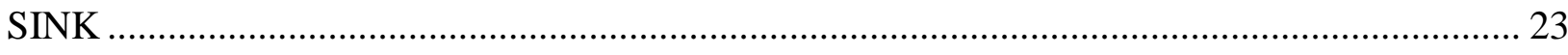

FIG.2.10. TEMPERATURE \& VELOCITY DISTRIBUTIONS AROUND TWO DIFFERENT CONFIGURATIONS OF ELLIPTICAL PIN FINS AT THREE DIFFERENT REYNOLDS NUMBERS WITH NANOFLUID AS COOLANT.

FIG.2.11. (a-f). EFFECT OF PIN ORIENTATION AND NANOFLUID VOLUME FRACTION ON NUSSELT NUMBER AT VARIOUS REYNOLDS NUMBERS, (a) NUSSELT NUMBER 
OF FIRST PIN, (b) NUSSELT NUMBER OF SECOND PIN, (c) NUSSELT NUMBER OF THIRD PIN, (d) NUSSELT NUMBER OF FORTH PIN, (e) NUSSELT NUMBER OF FIFTH PIN, (ef) OVERALL NUSSELT NUMBER OF HEAT SINKOF PIN ORIENTATION AND NANOFLUID VOLUME FRACTION ON NUSSELT NUMBER AT VARIOUS REYNOLDS NUMBERS 35

FIG.2.12. EFFECT OF PIN ORIENTATION AND NANOFLUID VOLUME FRACTION ON EULER NUMBER 37

FIG.3.1. SCHEMATIC OF THIN FILM EVAPORATION 39

FIG.3.2. DIMENSIONLESS MICROLAYER PROFILE \& FILM THICKNESS AT VARIOUS SUPERHEATS 49

FIG.3.3. DIMENSIONLESS MICROLAYER PROFILE \& HEAT FLUX AT VARIOUS SUPERHEATS 49

FIG.3.4. TEMPERATURE PROFILES AND PATHS FOR EVAPORATION .......................... 51

FIG.3.5. SUPERHEAT EFFECTS ON THIN FILM PROFILES ………………….................. 52

FIG.3.6. DIMENSIONLESS FILM THICKNESS, LIQUID, VAPOR, CAPILLARY AND

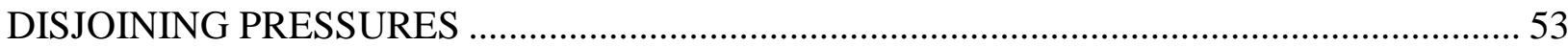

FIG.3.7. LIQUID PRESSURE DIFFERENCE AT VARIOUS SUPERHEATS ......................... 54

FIG.3.8. INTERFACE TEMPERATURE OF THE THIN FILM AT VARIOUS SUPERHEATS 54

FIG.3.9. CURVATURE PROFILES AT VARIOUS SUPERHEATS ......................................... 55

FIG.4.1. VAPOR CONDENSATION HEAT EXCHANGER .................................................. 56

FIG.4.2. VARIATIONS OF $u_{F}^{*}$ WITH DIMENSIONLESS FRONT LOCATION ................... 73

FIG.4.3. VARIATIONS OF $u_{F}^{*}$ WITH DIMENSIONLESS LOCATION ................................ 73

FIG.4.4. VARIATIONS OF $\delta_{F}^{*}$ WITH DIMENSIONLESS LOCATION …….......................... 74

FIG.4.5. VARIATIONS OF $u_{l}^{*}$ WITH DIMENSIONLESS LOCATION ................................... 75 
FIG.4.6. VARIATIONS OF $p_{v}^{*}$ WITH DIMENSIONLESS LOCATION

FIG.4.7. VARIATIONS OF $p_{v}^{*}$ WITH DIMENSIONLESS FRONT LOCATION 75

FIG.4.8. VARIATIONS OF $\theta_{T}$ WITH DIMENSIONLESS FRONT LOCATION 76

FIG.4.9. PROILES FOR FLUID TEMPERATURE, PRESSURE, SATURATION, AND THE VELOCITY OF THE VAPOR PHASE 77

FIG.5.1. CONDENSATION FLOW LENGTH IN TERMS OF TIME 93

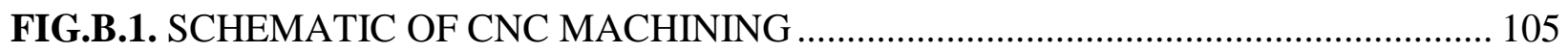

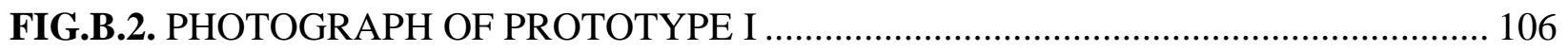

FIG.C.1. PHOTOGRAPH OF EXPERIMENTAL PROTOTYPE............................................. 107

FIG.C.2. PHOTOGRAPH OF CNC MACHINE PROTOTYPE............................................... 110

FIG.C.3. CONTOUR OF VELOCITY DISTRIBUTION ...................................................... 114

FIG.C.4. FOUR BOLTS TO TIGHTEN LEXAN SHEETS ………………………............ 117

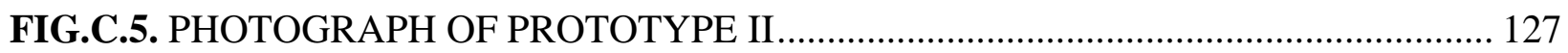




\section{LIST OA TABLES}

TABLE 2.1. EXPERIMENTAL DESGIN CACULATIONS ............................................... 20

TABLE 2.2. COMPARISON BETWEEN DATA OF KOSAR AND PELES [33] AND

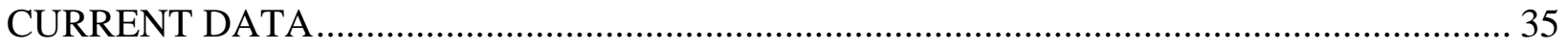

TABLE 2.3. COMPARISON BETWEEN RESULTS OF YANG ET AL [32] AND CURRENT

RESULTS 36

TABLE 3.1. PROPERTIES OF THE WORKING FLUID FC-72 ....................................... 47

TABLE 3.2. DIMENSIONAL PARAMETERS \& CALCULATED VALUES ........................ 49

TABLE 3.3. COMPARISON OF THE CALCULATED RESULTS WITH PREVIOUS

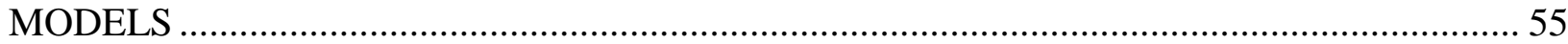

TABLE 5.1. PROPERTIES OF DIFFERENT POROUS MEDIUMS AND TWO WORKING

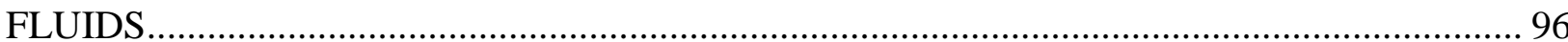




\section{LIST OF SYMBLES}

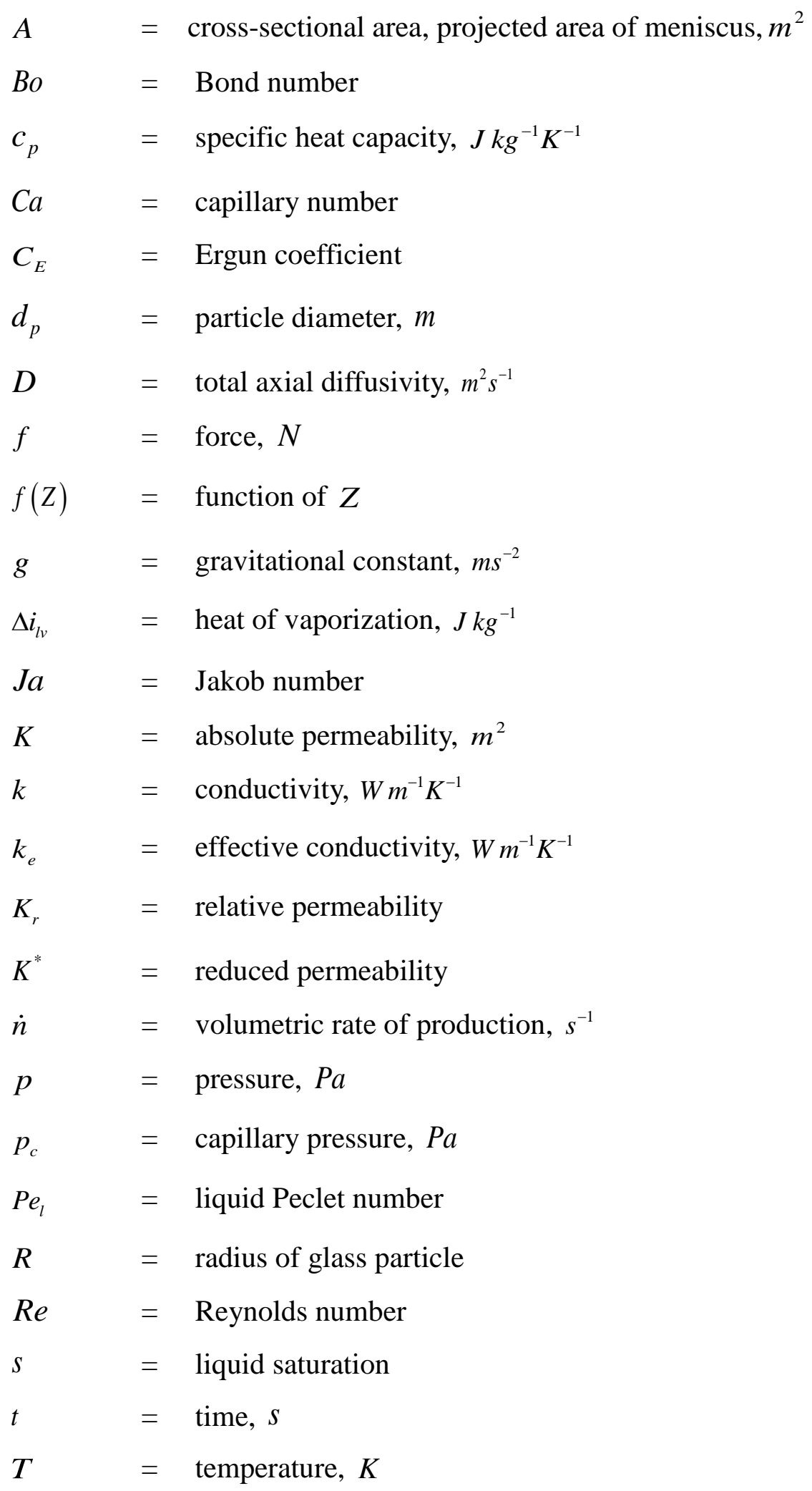




$$
\begin{array}{ll}
u & =\text { superficial velocity, } m^{-1} \\
u_{F} & =\text { condensation forepart velocity } \\
x & =\text { axial location, } m \\
r_{p} & =\text { pore radius, } m \\
r_{e f f} & =\text { effective pore radius, } m \\
R & =\text { radius of sphere at the contact line, } m \\
R e & =\text { Reynolds number, } \frac{\rho_{e f f} u D_{h}}{\mu_{e f f}} \\
s & =\text { saturation } \\
t & =\text { time, } s \\
T & =\text { temperature, } K \\
u & =\text { pore velocity, } m / s \\
Z & =\text { Darcy velocity, } u_{D}=u \varepsilon, m / s \\
u_{D} & =\text { volume, } m^{3} \\
V & =\text { length } \\
z & =\text { difference } \\
& =
\end{array}
$$

\section{Greek symbols}

$$
\begin{array}{ll}
\alpha_{l} & =\text { liquid diffusivity, } \mathrm{m}^{2} \mathrm{~s}^{-1} \\
\delta_{l} & =\text { thickness of liquid region, } \mathrm{m} \\
\delta_{l v} & =\text { thickness of two phase region, } \mathrm{m} \\
\varepsilon & =\text { porosity } \\
\mu & =\text { viscosity, } \mathrm{kg} \mathrm{m}^{-1} \mathrm{~s}^{-1} \\
\rho & =\text { density, } \mathrm{kg} \mathrm{m}^{-3} \\
\sigma & =\text { surface tension, } \mathrm{Nm}^{-1}
\end{array}
$$

\section{Subscripts}

$$
\begin{array}{ll}
\text { eff } & =\text { effective } \\
e & =\text { exit }
\end{array}
$$




$$
\begin{array}{ll}
F & =\text { forepart } \\
g & =\text { gas } \\
i & =\text { initial } \\
l & =\text { liquid } \\
m & =\text { modified } \\
s & =\text { solid } \\
s a t & =\text { thermodynamic saturation state } \\
t r & =\text { transition } \\
0 & =\text { inlet } \\
1 & =\text { first } \\
2 & =\text { second }
\end{array}
$$

\section{Superscript}

* $\quad=$ dimensionless




\title{
HEAT TRANSFER ENHANCEMENT OF VAPOR CONDENSATION \\ HEAT EXCHANGER
}

Husam H. Rajab

\section{Dr. Hongbin Ma, Dissertation Supervisor}

\begin{abstract}
A typical vapor condensation condenser consists of two major heat transfer processes, i.e., vapor condensation and forced convection. In order to enhance heat transfer, the condensation heat transfer utilizing the porous medium is investigated, and at the same time, the elliptical pin fin effect on the forced convection of nanofluid studied. The forced convective heat transfer on nanofluids in an elliptical pin-fin heat sink of two different pin orientations is numerically studied by using a finite volume method.
\end{abstract}

With increasing Reynolds number, the recirculation zones behind the pins increased. There were more recirculation zones for the pins with different angular orientations than for pins with the same angular orientation. It is observed that the Nusselt number for the pins with different angular orientations was higher than that for pins with the same angular orientation. The results show that with increasing volume fraction of nanoparticles and angular orientation of pins for a given Reynolds number, Euler and Nusselt numbers as well as overall heat transfer efficiency increase.

The non dimensional mass, momentum and energy equations based on non dimensional pressure, temperature, heat capacity, capillary, and bond numbers are developed for the vapor condensation occurring in the porous medium. The volumetric viscous force for the flow is described by Darcy's law. For the microscopic interfacial shear stress, a permeability term that relates flow rate and fluid physical properties (i.e. viscosity) to pressure gradient 
$K_{l}=\mu_{l}\left\langle u_{l}\right\rangle / \nabla\langle p\rangle^{l}$ is implemented. The effects of permeability, porosity, and effective pore radius parameters on porous medium performance are introduced.

The occurrence of thin liquid film inside pores is addressed and the Laplace-Young equation is depicted. In calculations the Darcy-Ergun momentum relation is implemented which provide accurate means to determine the capillary performance parameters of porous medium. The dimensionless thicknesses of the two-phase and liquid regions and embodiment of unique characteristics based on the total thermal diffusivity and absolute permeability are depicted. Therefore, analyses of the phase change and two-phase flow are made by defining regions, over which appropriate approximations are made. 


\section{CHAPTER ONE: INTRODUCTION}

\subsection{PRINCIPLES OF CONDENSATION AND EVAPORATION}

As shown in figure 1.1, in a vapor-to-condensate heat exchanger process, two streams of water- primary and steam-secondary are used. The primary cold water becomes hot by coming in direct contact with the heated from bottom surface (o-o'), while the secondary steam which is used as supply condensed water to the cooling tower, decreases its temperature by exchanging only sensible heat with the cooled and water stream (o-s). Thus vapor content of the supply steam remains constant in an indirect cooling system (Figure 1.2), while its temperature drops. Obviously, everything else remaining constant, the temperature drop obtained in a direct cooling system is larger compared to that obtained in an indirect system, in addition the direct cooling system is also simpler and hence, relatively inexpensive.

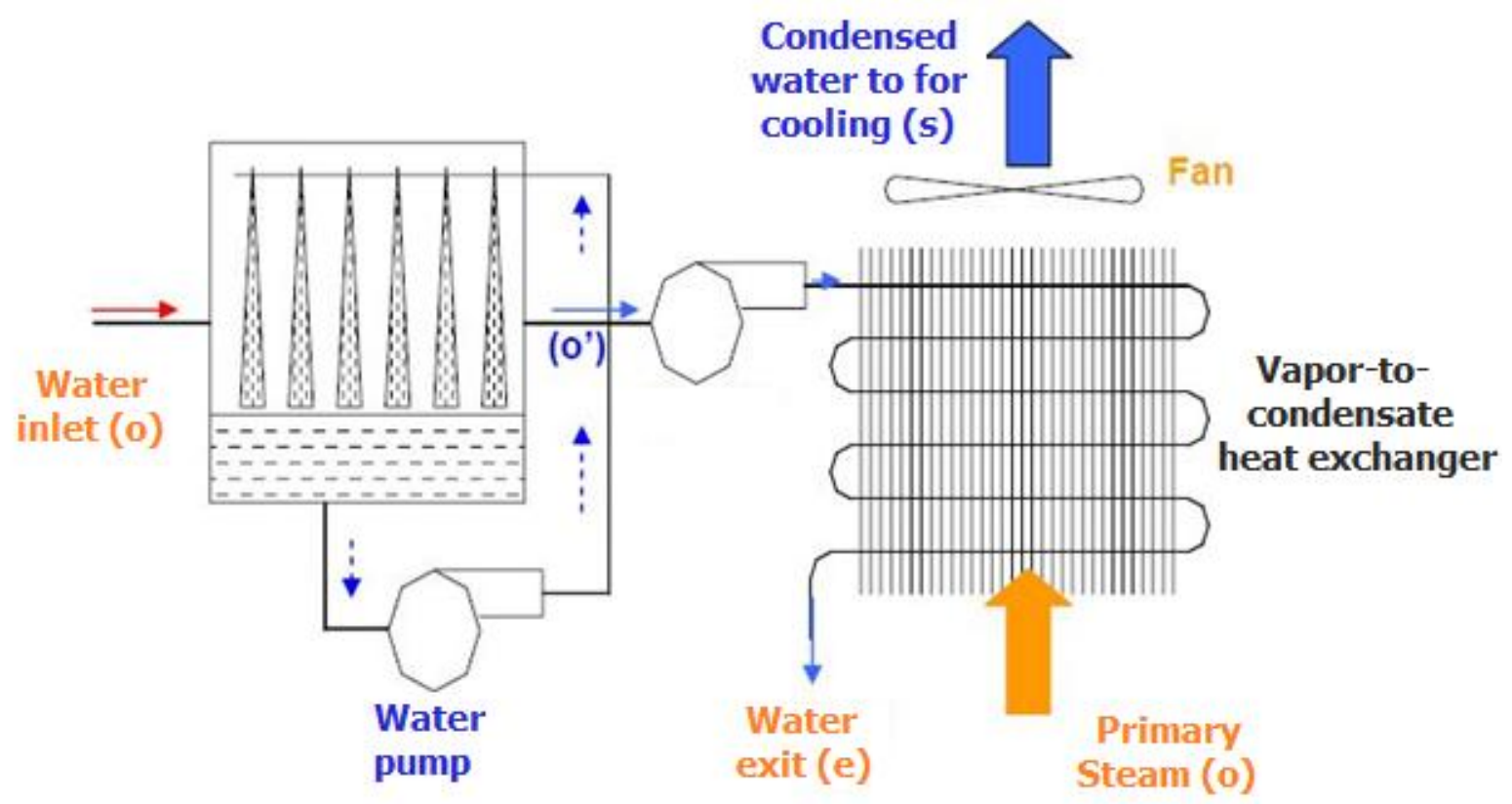

Fig 1.1. Vapor-to-condensate heat exchanger process 
However, since the steam content remains constant in an indirect process, this may provide greater degree of comfort in regions with higher vapor-to-condensate ratio. In modern day indirect coolers, the conditioned steam flows through tubes or plates made of non-corroding plastic materials such as polystyrene (PS) or polyvinyl chloride (PVC). On the outside of the plastic tubes or plates thin film of water is maintained. Water from the liquid film on the outside of the tubes or plates evaporates and cools through the tubes or plates sensibly. Even though the plastic materials used in these coolers have low thermal conductivity, the high external heat transfer coefficient due to evaporation of water makes up for this. The commercially available indirect coolers have saturation efficiency as high as $80 \%$.

\subsection{COOLING TOWER MATERIALS SELECTION}

If pre-cooling the dry steam is the objective then a wet media pad should be used to cool the dry steam before it reaches the condenser coils. In this case the type of media used is of greatest importance. The important characteristics when choosing a material are the pressure

drop through it, how well it condenses the steam or cooling efficiency, and how it holds up to water damage. Water damage will deteriorate the material's performance because of salts deposits and mold formation. This will lower the cooling efficiency and increase the pressure drop. Another consideration that should not be neglected when selecting the material is the cost. This is very important when analyzing a system's economic advantage. 


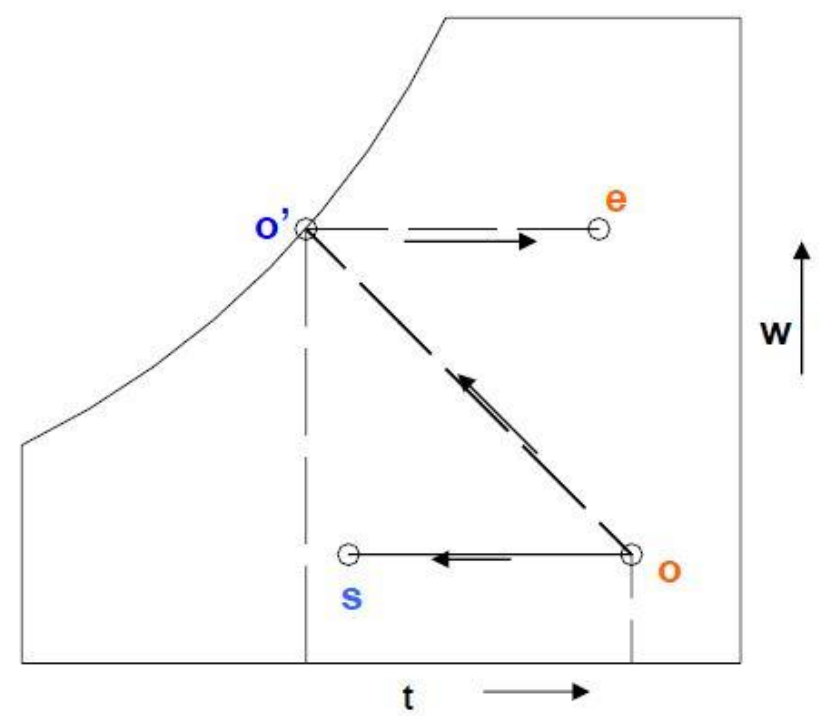

Fig 1.2. Direct \& Indirect Cooling System

\subsection{IDENTIFYING STEAM POWER SYSTEMS}

Currently nine out of ten power plants in the United States that generate electricity from steam power (Fig. 1.3) require condensate cooling. These systems are categorized as either oncethrough or wet-recirculation. Once-through cooling systems discharge water directly after it has absorbed system heat. Wet-recirculating systems (wet-cooling) operate in a closed loop where a considerable amount of water is lost in the cooling towers through evaporation cooling. The remaining power plants use air for heat removal in a process called dry-cooling. This process reduces water consumption by more than $90 \%$.

However, air as a lower heat capacity than water making this design less efficient resulting in significant increases in size and cost. In summer, when electricity demand peaks, ambient temperature increases, this significantly decreases the temperature difference between steam and ambient air resulting in a decrease of cooling capacity. In order to significantly reduce or eliminate the use of water for cooling power plants, a highly efficient heat exchanger for the vapor condensation is needed. 


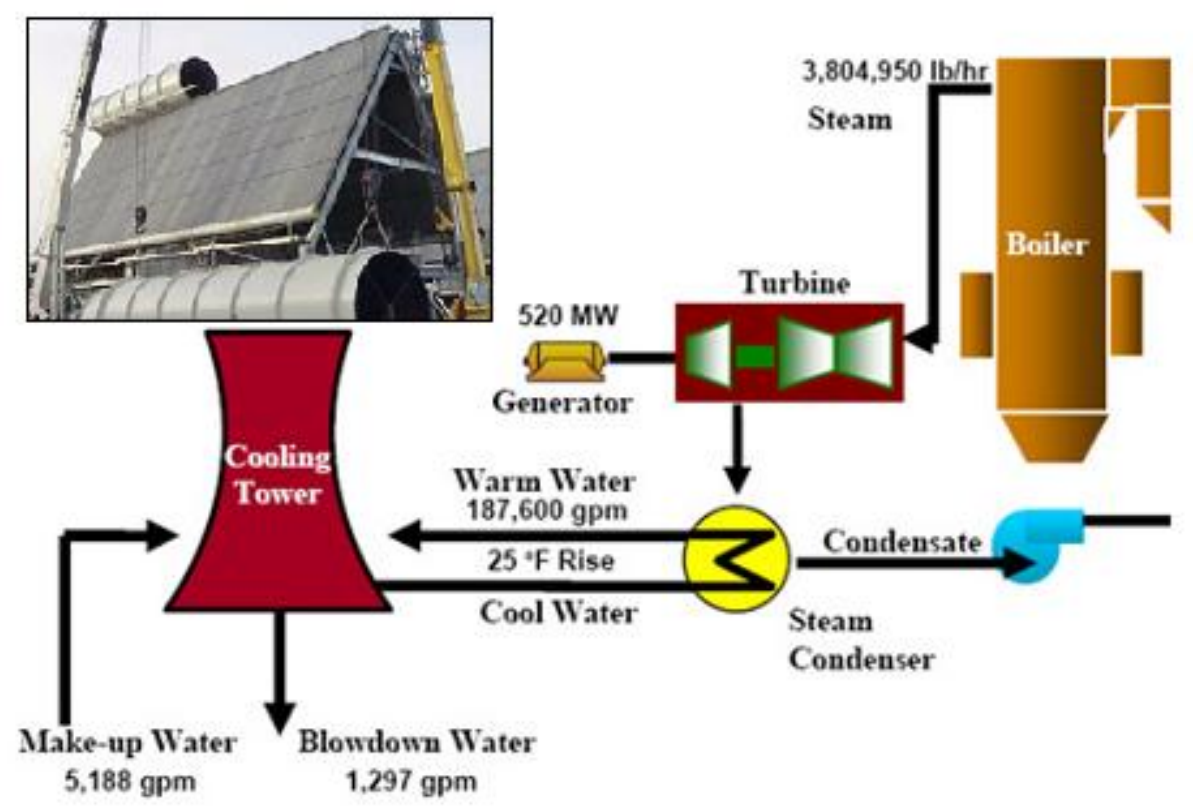

Figure 1.3. Water flow in once-through cooling

\subsection{OBJECTIVES \& RESEARCH APPROACH}

For a typical vapor condensation heat exchanger, as shown in Fig. 1.4, the steam from the power plant is condensed inside the heat exchanger. The heat released from the condensation is transferred through the exchanger wall and removed by the forced convection. In order to enhance heat transfer of the condensation heat exchanger, the condensation heat transfer occurring inside the heat exchanger is needed to be increased and the forced convection heat transfer is needed to be enhanced as well. In the current investigation, the heat transfer enhancement of forced convection using elliptical pin fins is investigated. At the same time, the vapor condensation occurring in the porous medium addressed. 


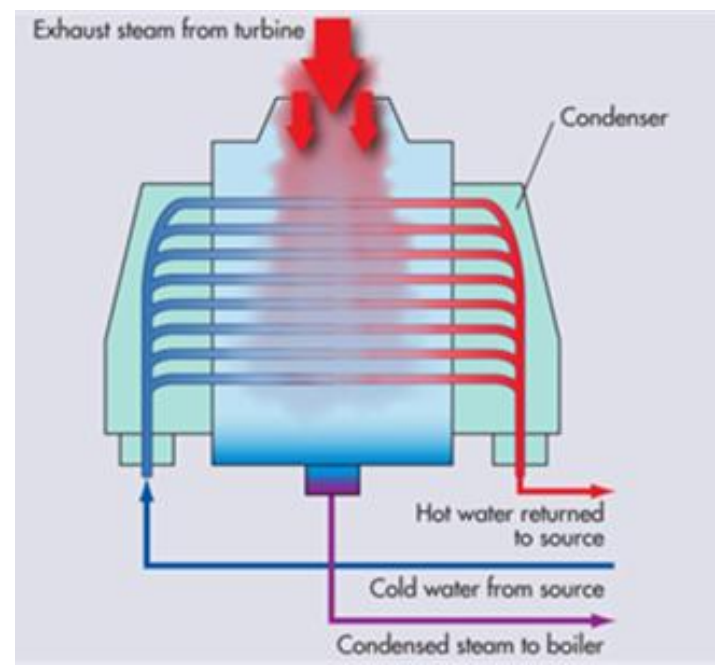

Figure 1.4. Typical Vapor Condensation System

In order to increase the heat transfer rate of the vapor condensation heat exchanger, the current investigation will focus on the condensation heat transfer and forced convection. For the vapor condensation, in order to increase the condensation heat transfer rate, porous medium is utilized to increase the condensation area, and at the same time, the condensate can be effectively removed by the capillary force. The heat released from the condensation must be efficiently removed for the forced convection. In order to increase the heat transfer coefficient of forced convection, the elliptical pin fins with nanofluid is investigated. 


\section{CHAPTER TWO: HEATTRANSFER ENHANCEMENT USING MINI/MICRO ELLIPTICAL PIN-FIN HEAT SINKS WITH NANOFLUIDS}

\subsection{INTRODUCTION}

In recent years, nanofluids (NFs) have proven to have a great potential for enhancing the heat transport capability of heat transfer devices [1-8]. Therefore, using nanofluids as a working fluid is well suited for use in high performance compact heat exchangers and heat sinks used in electronic equipment. One of the important characteristics of nanofluids is represented by their higher thermal conductivities with respect to conventional coolants. The enhancement of thermal conductivity of NFs depends on particle diameter and volume fraction, thermal conductivities of base fluid and nanoparticles as well as Brownian motion of nanoparticles, which is a key mechanism in thermal conductivity enhancement. Several correlations have been developed regarding the thermal conductivity of copper oxide and water nanofluids [9-18]. Wang and Mujumdar [9] gave a quality review of such correlations. The Hamilton-Crosser (HC) model [10] and Maxwell model [11] include the effects of distribution and the interfacial layer at the particle/liquid interface along with the Brownian motion of nanoparticles, which are some key mechanisms of thermal conductivity enhancement. Koo and Kleinstreuer [12] proposed a thermal conductivity model that takes into account the effect of temperature, particle volumetric concentration, and properties of base fluid as well as nanoparticles subjected to Brownian motion. Comparisons between models $[13,14]$ show that Koo and Kleinstreuer's model better captured the results of an experimental study by Namburu et al. [15], and it predicted thermal conductivity of nanofluids better than other available models [16-18].

Many experimental and numerical studies in the literature focus on heat transfer enhancement of pin shapes and their angular orientation in pin-fin heat sinks and pin fin arrays 
[19-21]. Sparrow and Vemuri [19] have shown that fin array and its orientation have a positive influence in heat transfer. Rubio-Jimenez et al. [20] analyzed the effect of shape of pin on temperature distribution and pressure drop in micro pin-fin heat sinks. The results showed that the fin shape plays an important role in the pressure drop rather than heat dissipation. They showed that the best performance can be obtained with flat-shaped fins. Huang and Sheu [21] studied fluid flow in micro heat sink so as to obtain temperature field and distribution of Nusselt number on the square micro-pin-fin heat sink for the steady incompressible flow of Newtonian fluids. They found that the averaged value of Nusselt number increases with increasing Prandtl and Reynolds numbers.

Investigations into the optimization of geometrical structures of micro/mini heat sinks, and the use of nanofluids in cooling devices for cooling electronic equipment are still embryonic; much more study is required in order to better understand the thermal and fluid dynamic characteristics of these devices with this very promising new family of coolants and different geometries. Therefore, in the current investigation, an analysis was conducted to determine the effect of nanofluid on the heat transfer performance in an elliptical mini pin-fin heat sink including the influence of pin orientation. An effective thermal conductivity model, which takes into account the mean diameter of nanoparticles and Brownian motion,was used in the calculations. In order to compare the results, one heat sink has pins with a constant orientation angle, and the other heat sinkhas pins with varied orientation angles from 0 degree for the first pin to 90 degrees for the last pin.

\subsection{MATHEMATICAL MODEL AND GOVERNING EQUATIONS}

Conjugated heat transfer in a nanofluid-cooled pin-fin heat sink was a major focus in this study including the effect of heat transfer enhancement of suspensions containing nanoparticles. 
Figure 2.1 shows the schematic diagram of a pin-fin heat sink with variable orientation angles. The nanofluids studied here consisted of water and $\mathrm{CuO}$ nanoparticles with three different volume fractions. In order to be able to use the single phase approach, the diameters of nanoparticles were assumed to be less than $100 \mathrm{~nm}$ (ultrafine solid particle). For Reynolds numbers of less than 1000, Zukauskas [22] observed that fluid flow around a tube bank can be considered to be dominantly laminar. The flow was assumed to be incompressible; hence, radiation and compressibility effects were neglected in this study.
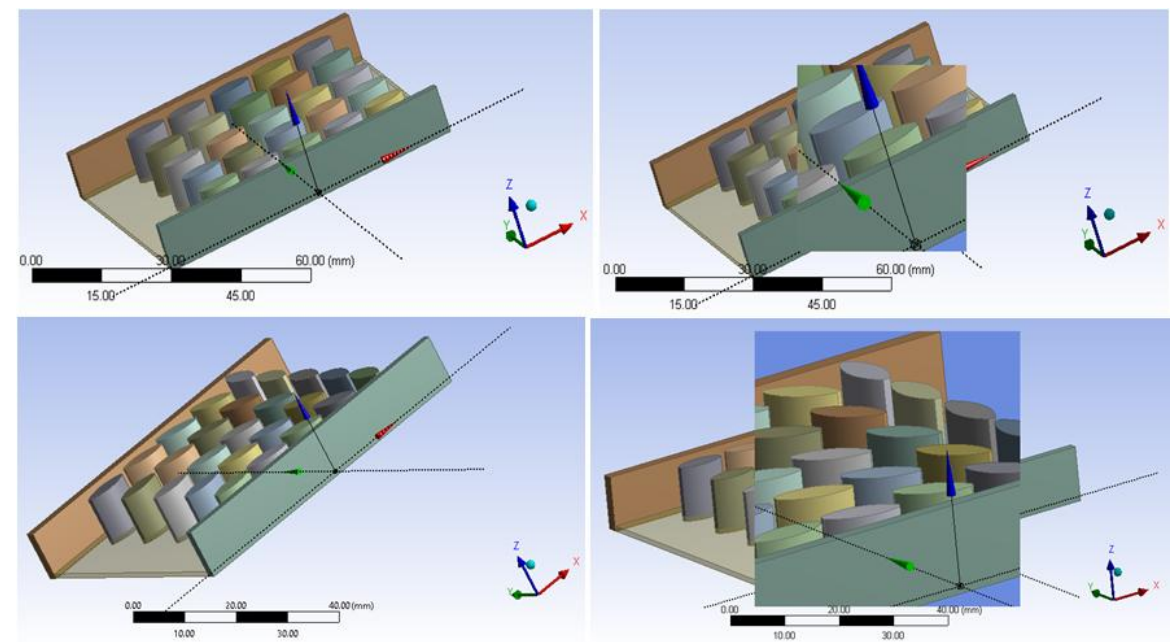

Figure 2.1. Schematic of the elliptical pin-fin heat sink. (Left: pins arranged at the same angle; Right: pins arranged at different angles; $0^{\circ}, 22.5^{\circ}, 45^{\circ}, 67.5^{\circ}$, and $90^{\circ}$ )

The governing equations for an incompressible Newtonian liquid in the laminar regime and in steady state conditions are given by the following.

continuity equation:

$$
\frac{\partial u}{\partial x}+\frac{\partial v}{\partial y}+\frac{\partial w}{\partial z}=0
$$

X-momentum equation:

$$
\rho_{\text {eff }}\left(u \frac{\partial u}{\partial x}+v \frac{\partial u}{\partial y}+w \frac{\partial u}{\partial z}\right)=-\frac{\partial p}{\partial x}+\frac{\partial}{\partial x}\left(\mu_{\text {eff }} \frac{\partial u}{\partial x}\right)+\frac{\partial}{\partial y}\left(\mu_{\text {eff }} \frac{\partial u}{\partial y}\right)+\frac{\partial}{\partial z}\left(\mu_{\text {eff }} \frac{\partial u}{\partial z}\right)
$$


y-momentum equation:

$$
\rho_{\text {eff }}\left(u \frac{\partial v}{\partial x}+v \frac{\partial v}{\partial y}+w \frac{\partial v}{\partial z}\right)=-\frac{\partial p}{\partial y}+\frac{\partial}{\partial x}\left(\mu_{e f f} \frac{\partial v}{\partial x}\right)+\frac{\partial}{\partial y}\left(\mu_{e f f} \frac{\partial v}{\partial y}\right)+\frac{\partial}{\partial z}\left(\mu_{e f f} \frac{\partial v}{\partial z}\right)
$$

z-momentum equation:

$$
\rho_{\text {eff }}\left(u \frac{\partial w}{\partial x}+v \frac{\partial w}{\partial y}+w \frac{\partial w}{\partial z}\right)=-\frac{\partial p}{\partial z}+\frac{\partial}{\partial x}\left(\mu_{e f f} \frac{\partial w}{\partial x}\right)+\frac{\partial}{\partial y}\left(\mu_{e f f} \frac{\partial w}{\partial y}\right)+\frac{\partial}{\partial z}\left(\mu_{e f f} \frac{\partial w}{\partial z}\right)
$$

energy:

$$
\left(\rho C_{p}\right)_{\text {eff }}\left(u \frac{\partial T}{\partial x}+v \frac{\partial T}{\partial y}+w \frac{\partial T}{\partial z}\right)=\frac{\partial}{\partial x}\left(k_{\text {eff }} \frac{\partial T}{\partial x}\right)+\frac{\partial}{\partial y}\left(k_{\text {eff }} \frac{\partial T}{\partial y}\right)+\frac{\partial}{\partial z}\left(k_{\text {eff }} \frac{\partial T}{\partial z}\right)+\psi^{2}
$$

where, $u, v, w$ are velocity components in $x, y$ and $z$ directions, respectively. $T$ and $P$ are temperature and pressure, respectively. $\rho_{\text {eff }}$ and $C_{p, e f f}$ are effective density and specific heat of nanofluid. $\psi^{2}$ is the viscous dissipation term, and it represents the time rate at which energy is being dissipated per unit volume through the action of viscosity. For an incompressible flow, it is written as follows:

$$
\psi=\sqrt{\left\{\begin{array}{c}
2\left[\left(\mu_{\text {eff }} \frac{\partial u}{\partial x}\right)^{2}+\left(\mu_{\text {eff }} \frac{\partial v}{\partial y}\right)^{2}+\left(\mu_{\text {eff }} \frac{\partial w}{\partial z}\right)^{2}\right]+\left(\mu_{\text {eff }} \frac{\partial u}{\partial y}+\mu_{\text {eff }} \frac{\partial v}{\partial x}\right)^{2} \\
+\left(\mu_{\text {eff }} \frac{\partial v}{\partial z}+\mu_{\text {eff }} \frac{\partial w}{\partial y}\right)^{2}+\left(\mu_{\text {eff }} \frac{\partial w}{\partial x}+\mu_{\text {eff }} \frac{\partial u}{\partial z}\right)^{2}
\end{array}\right\}}
$$

The effective density and heat capacity of nanofluid can be expressed by the classical model $[23,24]$ as:

$$
\begin{gathered}
\rho_{e f f}=(1-\alpha) \rho_{f}+\alpha \rho_{p} \\
\left(\rho C_{p}\right)_{e f f}=(1-\alpha)\left(\rho C_{p}\right)_{f}+\alpha\left(\rho C_{p}\right)_{p}
\end{gathered}
$$


Where $\alpha$ and $C_{p}$ and $\rho$ are volume fraction, heat capacity and density, respectively. Indexes $f$ and $p$ refer to fluid and solid, respectively.
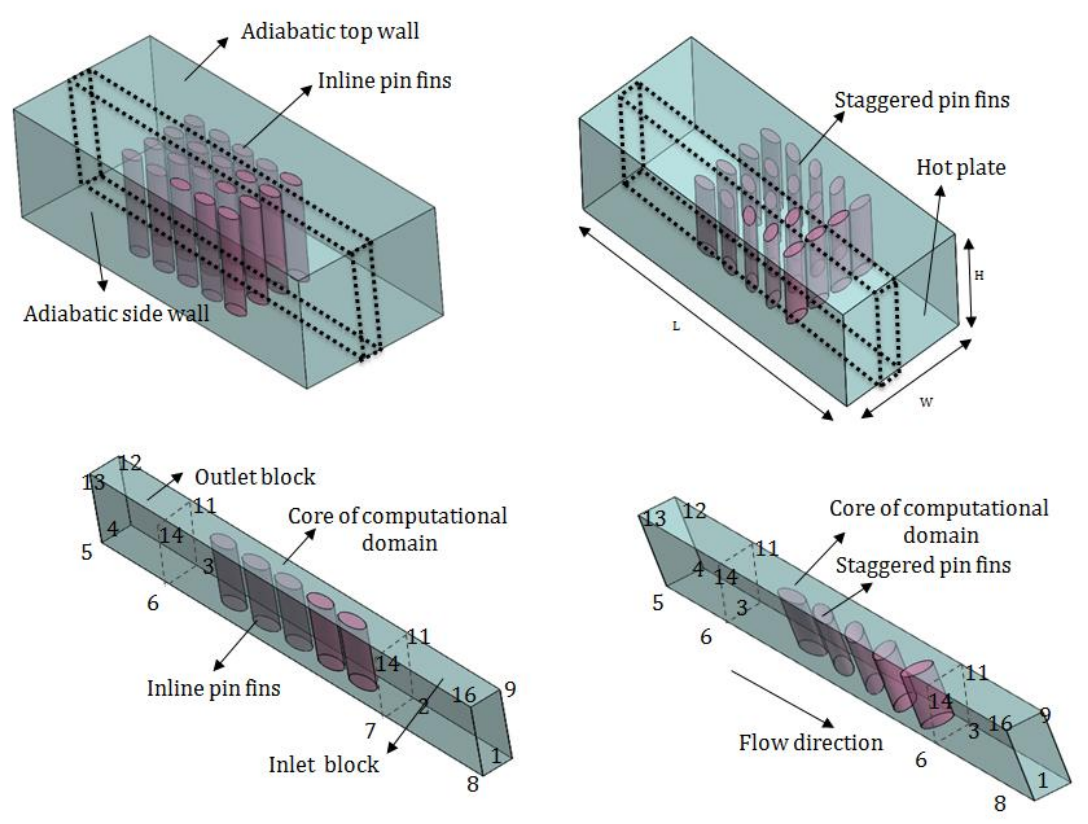

Figure 2.2 (a) Computational Domain

Using the correlation given by Koo and Kleinstreuer [25, 26]. This model takes into account the effect of Brownian motion, temperature, mean diameter and volume fraction of nanoparticle, and nanoparticle on nanofluid thermal performance. Furthermore, Li and Kleinstreuer [28] compared this model with the MSBM model by Prasher et al. [13] for two different nanofluids, $\mathrm{CuO}$-water and $\mathrm{Al}_{2} \mathrm{O}_{3}$-water and found that it can predict thermal conductivity of nanofluid accurately up to a volume fraction of $4 \%$.

Based on KKL model $[25,26]$ the effective thermal conductivity of nanofluid is in the following form:

$$
k_{\text {eff }}=k_{\text {static }}+k_{\text {brownian }}
$$


The first term is the conventional static part which is the well-known Hamilton Crosser's equation [10] and can be defined as:

$$
k_{\text {static }}=k_{f}\left[\frac{\left(k_{p}+2 k_{f}\right)-2 \alpha\left(k_{f}-k_{p}\right)}{\left(k_{p}+2 k_{f}\right)+\alpha\left(k_{f}-k_{p}\right)}\right]
$$

Major enhancement in thermal performance of nanofluid is due to Brownian motion associated with nanoparticles. The second term in an effective thermal conductivity model is the dynamic part which originates from the particle Brownian motion and can be expressed as:

$$
k_{\text {brownian }}=5 \times 10^{4} \beta \alpha \rho_{f} C_{p, f} \sqrt{\kappa_{b} T / d_{p} \rho_{p}} f(T, \alpha)
$$

where $\alpha, d_{p}$ and $\rho_{p}$ are nanoparticle volume fraction, nanoparticle mean diameter, and density of nanoparticle, respectively. $\kappa_{b}$ is Boltzmann constant, $\left(1.3807 \times 10^{-23} j / K\right)$. The function $f$ and $\beta$ , are to be determined semi-empirically. The model parameter $f$ considers the augmented temperature dependent is from Hamilton Crosser's equation [10] and it is due to particle interactions, and function $\beta$ represents the fraction of the liquid volume which travels with particles and decreases with the particles' volumetric concentration due to the viscous effect of moving particles. The functions $f$ and $\beta[26,27]$ can be combined to a new g-function which considers the influence of multi-particle interaction which depends on volume fraction, temperature and particle diameter [29],

$$
g=\left(\begin{array}{l}
a+b \ln \left(d_{p}\right)+c \ln (\alpha) \\
+d \ln (\alpha) \ln \left(d_{p}\right)+e \ln \left(d_{p}\right)^{2}
\end{array}\right) \ln (T)+\left(\begin{array}{l}
m+h \ln \left(d_{p}\right)+i \ln (\alpha) \\
+j \ln (\alpha) \ln \left(d_{p}\right)+k \ln \left(d_{p}\right)^{2}
\end{array}\right)
$$

where $a, b, c, d, e, g, h, i, j, k$ are constants which are dependent of type of nanoparticles and base fluid. 
In order to take into account the thermal interfacial resistance $\left(\boldsymbol{R}_{b}\right)$ or Kapitza resistance [29] in the static part of the effective thermal model,

$$
R_{b}+\frac{d_{p}}{k_{p}}=\frac{d_{p}}{k_{p, e f f}}
$$

In the present study, we chose the value of $R_{b}=4 \times 10^{-8} \frac{\mathrm{km}^{2}}{\mathrm{~W}}$ according to Li \& Kleinstreuer [28]. The temperature dependent viscosity and thermal conductivity of pure water are as follows [30]:

$$
\begin{aligned}
& \mu_{f}=2.761 \times 10^{-6} \exp \left(\frac{1713}{T}\right) \\
& k_{f}=0.6\left(1+4.167 \times 10^{-5} T\right)
\end{aligned}
$$

In this study a combination of wall, inlet, outlet and symmetry boundary conditions were applied to the computational domain. All walls in contact with liquid flow were treated as no-slip boundary conditions. A constant and uniform velocity and temperature distribution were applied to the inlets of hot and cold channels. At outlets, the static pressure was fixed, and the remaining flow variables were extrapolated from the interior of the computational domain. At solid-liquid interface, the temperature continuity must be satisfied so the heat fluxes at interfaces are used to relate the temperatures to each other.

\subsection{SOLUTION AND PREDICTION PROCEDURE}

Equations (1-5) are solved separately using a finite volume code based on a collocated grid system. The conservation equations are discretized by means of the finite volume method in a collocated grid based on SIMPLE algorithm [31]. The diffusive and convective terms are discretized using second order centered and QUICK schemes [32], respectively. In order to avoid velocity-pressure decoupling problems, the velocity components in the discretized continuity 
equation are calculated using an interpolation technique. The convergence of code was declared when the residual of each component of velocity vector, pressure and temperature become $10^{-7}$, $10^{-5}$, and $10^{-11}$, respectively.

After solving the governing equations for $u, v, w \& \mathrm{~T}_{f}$, other useful quantities such as Euler and Nusselt numbers were determined. The dimensionless pressure drop is presented by the Euler number [21], i.e. $E u=\frac{2 \Delta p}{\rho_{f m} U_{m}{ }^{2} N}$, where $\rho_{f m}$ is mean fluid density, $N$ is number of pin rows and $U_{m}$ is mean velocity in the minimum cross-section. The heat transfer rate from the hot wall can be calculated by $q=\left(\rho c_{p}\right)_{n f} u_{\text {in }} A_{i n} .\left(T_{\text {out }}-T_{i n}\right) / A_{h}$ where $A_{\text {in }}$ and $A_{h}$ denote the area of inlet and the base area of hot wall, respectively. $u_{i n}$ is the inlet velocity, and $T_{\text {out }}$ and $T_{\text {in }}$ are outlet and inlet bulk fluid temperatures, respectively. The convective heat transfer coefficient is defined as $h=q / \Delta T_{l m}$, where $\Delta T_{l m}$ is the $\log$ mean temperature difference , i.e., $\Delta T_{l m}=\left(T_{s}-T_{\text {in }}\right)-\left(T_{s}-T_{\text {out }}\right) / \ln \left(\frac{T_{s}-T_{\text {in }}}{T_{s}-T_{\text {out }}}\right)$ where $T_{s}$ is surface temperature.

Finally, the overall Nusselt number of the pin-fin heat sink is defined as follows, $N u=h D_{h} / k_{f}$. For performance assessment of the investigated pin-fin heat sink, the overall heat transfer efficiency is represented by the ratio of Nusselt number to the dimensionless pressure drop, i.e., $\gamma=N u / E u$. This definition relates the hydrodynamic and thermal performance, which allows us to obtain an indication about the overall pin-fin heat sink performance. $\gamma$ is also reasonable to evaluate performance of pin-fin heat sink with nanofluid, since nanofluid usually increases both pressure drop and total heat transfer.

\subsection{GRID INDEPENDENCY AND CODE VALIDATION}


The computational domain (Fig 2.2.a) was spatially discretized using two structure grids for inlet and outlet blocks and an unstructured grid of tetrahedral volume elements for the central region, which contained pins (Fig 2.2.b). A fine grid was used in regions with steep velocity and temperature gradient. Four grids with different sizes of 12,400 (coarse), and 154,000 (intermediate), and 1,536,000 (fine), and 2,890,000 (very fine) were used for the study of grid independency. The calculation results show a maximum difference of less than $0.025 \%$ in the computed results between the fine and very fine grids; hence, the fine grid was selected to conduct the calculation.

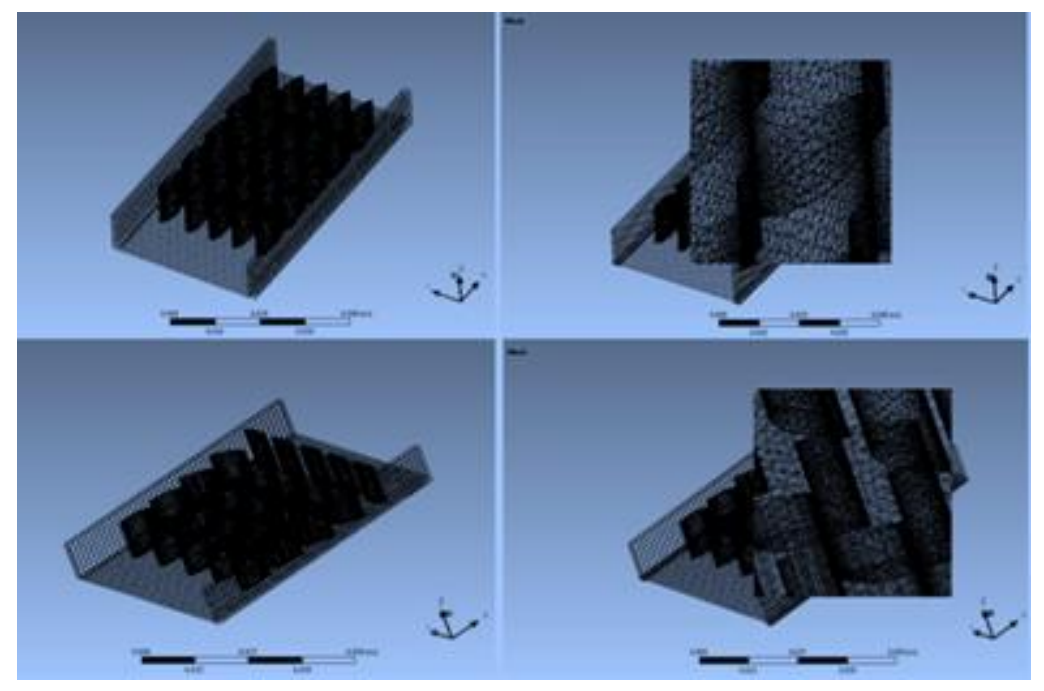

Figure 2.2.(b). Nonuniform computational grid: (Top): pins have same orientation angles, and (Bottom): pins have different orientation angles.

A number of researchers [32-33] used the same properties models and approach we used for nanofluid modeling and they found excellent agreement with experimental data. However, for further validation, we compared our results with experimental results by Yang et al [32] and validation of the code was also performed with respect to the experimental results presented by Kosar and Peles [33] for circular micro pin-fin heat sink with water as coolant. For each experiment, we have simulated only one symmetrical part of pin-fin heat sink, which was used in 
the experimental work of Kosar and Peles [33], and we also used an elliptical pin-fin heat sink, which was used in another experimental study by Yang et al [32] with the same boundary conditions in the experiments. The hydrodynamic and thermal boundary conditions used in experiments are constant velocity at the inlet of heat sinks, which is obtained from the inlet Reynolds number, and uniform and constant heat flux subjected to the bottom wall of heat sink. Furthermore, the channel and circular/elliptical pin fin surfaces are treated as no-slip boundary conditions, and at the channel outlet, the static pressure is fixed to atmospheric pressure, and the remaining flow variables are extrapolated from the interior of the computational domain. Tables 2.2 and 2.3 are to compare results from literature with present results. As seen, there is excellent agreement between the results of the calculations and previous studies in the literature.

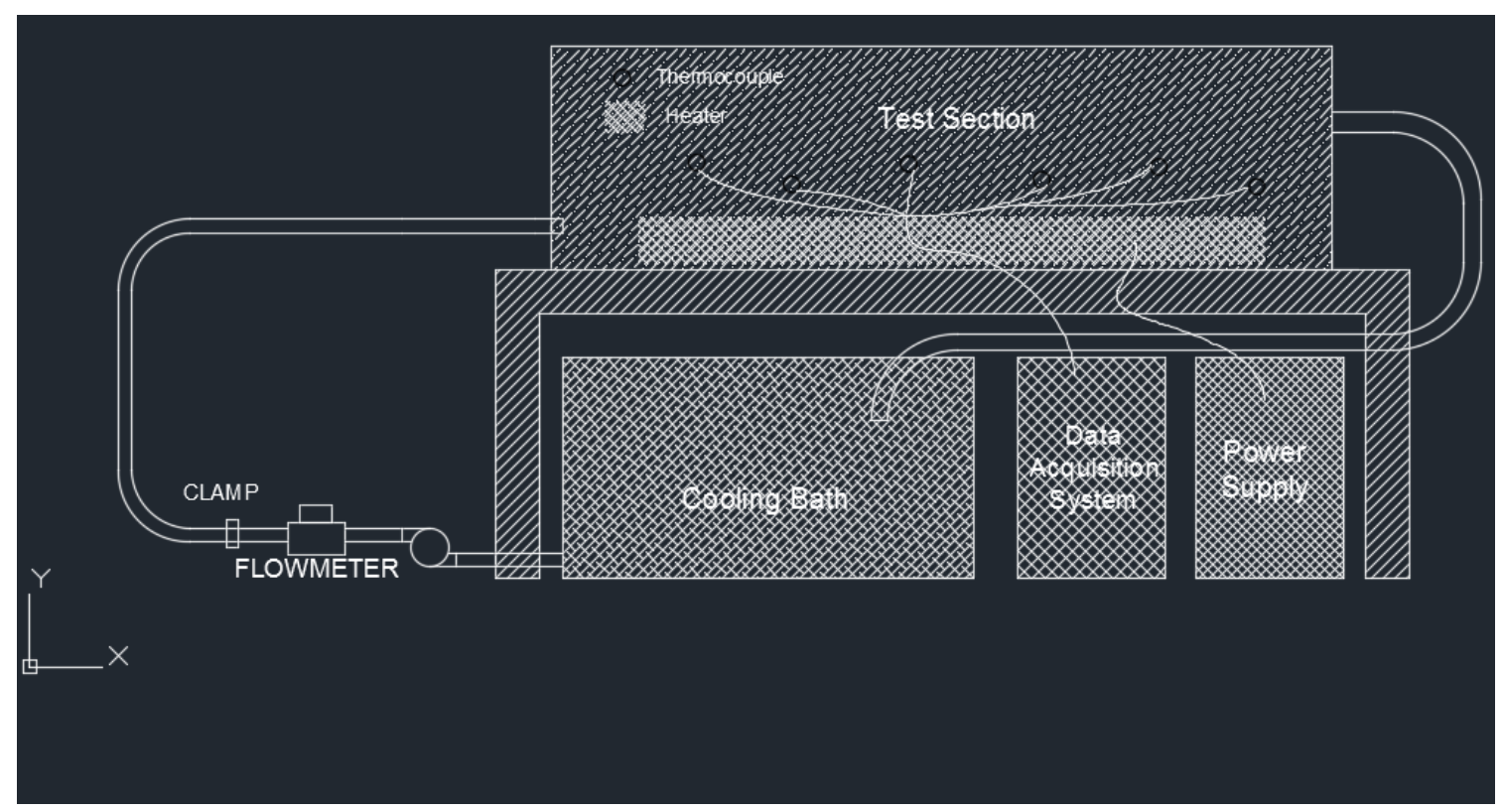

Figure 2.3. Schematic of experimental setup

\subsection{EXPERIMENTAL DESIGN}

A schematic of the experimental setup is shown in fig. 2.3. It mainly consists of the test section, the power supply, thermocouples for temperature measurement, a flow meter, a cooling bath, and the data acquisition system. The inlet velocities range from $0.02 \mathrm{~m} / \mathrm{s}$ up to $5.00 \mathrm{~m} / \mathrm{s}$ 
. The creation of the three dimensional pin fin heat sinks is achieved using additive processes. Hence, additive manufacturing of making three dimensional pin fin heat sinks prototype is utilized. In this additive manufacturing process, pin fin heat sink prototype (I) (Fig 2.4) is created by laying down successive layers of material until the heat sink is created. Each of these layers can be seen as a thinly sliced horizontal cross-section of the eventual pin fin heat sink. Two materials of pin fin heat sinks were tested; namely, PLA (Polylactic Acid) and ABS (Acrylonitrile Butadiene Styrene). Both materials showed good performance. However, ABS outperformed PLA in both stability and toughness. ABS material outweighed PLA in that ABS was less brittle. ABS was post-processed with acetone to provide a glossy finish.

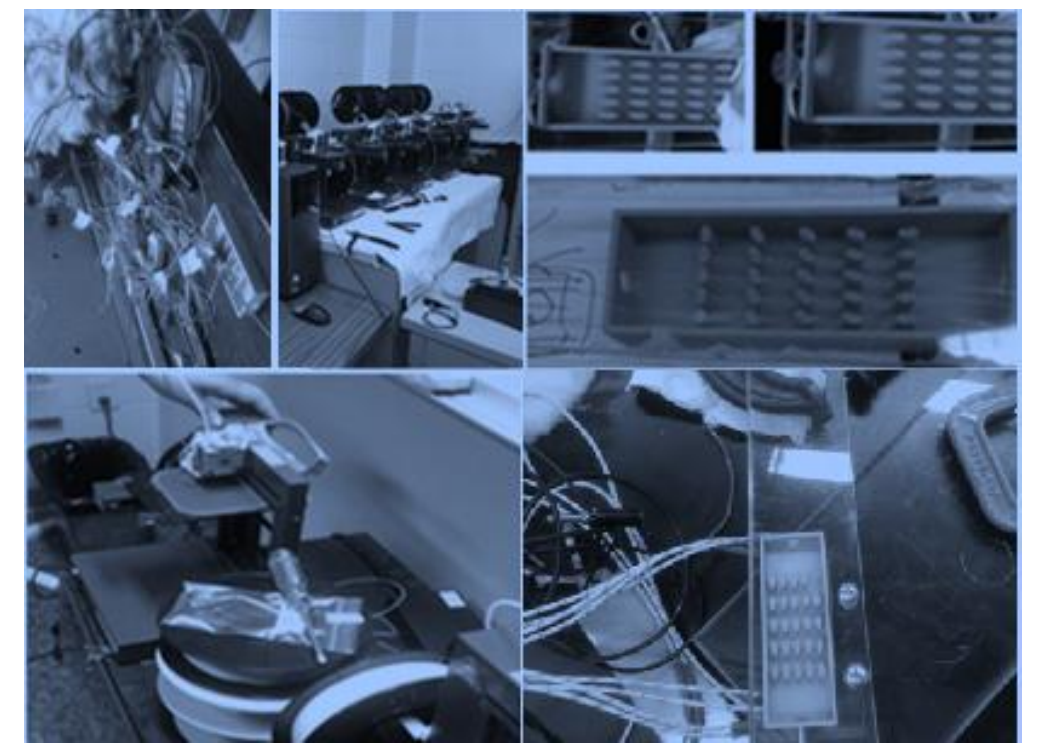

Figure 2.4. Production of MPFHS, Prototype (I)

Water flow with a uniform velocity, $v_{D}$ and with a bulk temperature. The test section containing the fin assembly is wrapped with wool sheet insulation so that the rate of heat loss to the surroundings is so small and can be ignored. The rate of heat dissipation from the fins and its surfaces to the flowing water is mainly convection. The total rate of heat transfer by convection from the fins and its surfaces may be expressed as 


$$
q_{t}=N \eta_{f} h A_{f} \theta_{b}+h A_{b} \theta_{b}
$$

where $h$ is the convection coefficient for the fins and its surfaces and $\eta_{f}$ is the efficiency of a single fin. Hence,

$$
\eta_{f}=\frac{\mathrm{M} \tanh m L}{h P L \theta_{b}}
$$

Eq. (16) can also be expressed as

$$
q_{t}=h\left[N \eta_{f} A_{f}+\left(A_{t}-N A_{f}\right)\right] \theta_{b}
$$

Rearranging yields,

$$
q_{t}=h A_{t}\left[1-\frac{N A_{f}}{A_{t}}\left(1-\eta_{f}\right)\right] \theta_{b}
$$

where $\theta_{b}$ is temperature difference $\left(\theta_{b}=T_{b}-T_{\infty}\right)$, the fin heat rate is expressed as

$$
q_{f}=M \tanh \left(m^{*} L\right)
$$

where

$$
M=\theta_{b}\left(h P k_{C u} A_{c}\right)^{1 / 2}
$$

and

$$
m=\left(h P / k_{C u} A_{c}\right)^{1 / 2}
$$

$A_{c}$ is the fin cross sectional area $A_{c}=\pi D^{2} / 4$. The thermal conductivity of fin material is evaluated at the average temperature of the fin surface and that for the water it is evaluated at the average temperature of entering and leaving water to/from the fins. The logarithmic mean temperature difference is expressed as, 


$$
\Delta T_{l m}=\frac{\left(T_{s}-T_{m, e}\right)-\left(T_{s}-T_{m, i}\right)}{\ln \frac{T_{s}-T_{m, e}}{T_{s}-T_{m, i}}}
$$

The overall Nusselt number of the pin fin heat sink is defined as,

$$
N u_{D}=0.3+\frac{0.62\left(\operatorname{Re} e_{D}\right)^{1 / 2}(\operatorname{Pr})^{1 / 3}}{\left(\left(1+\frac{0.4}{P r}\right)^{2 / 3}\right)^{1 / 4}}\left(1+\frac{\operatorname{Re}_{D}}{282000}\right)^{5 / 8}
$$

And the convective heat transfer is as expressed as,

$$
q_{\text {conv }}=\dot{m} C_{p}\left(T_{m, e}-T_{m, i}\right)
$$

The flow in the heat sink is completely enclosed, and the goal is to determine how the log mean temperature difference $\Delta T_{l m}$ varies with position along the heat sink and how the total convection heat transfer $q_{\text {conv }}$ is related to the difference in temperatures at the heat sink inlet and outlet $\left(T_{m, e}-T_{m, i}\right)$.

ABS provided a good mechanical toughness, and ease of fabrication, but not withstand high heat flux temperatures. Hence, a new pin heat sink prototyping was created (Fig 2.5) by cnc metal injection molding (CMIM). CMIM provided a balanced combination of wide temperature range, good dimensional stability, heat and pressure resistance, and electrical insulating properties. A description of the CMIM machining is given in the appendix. 


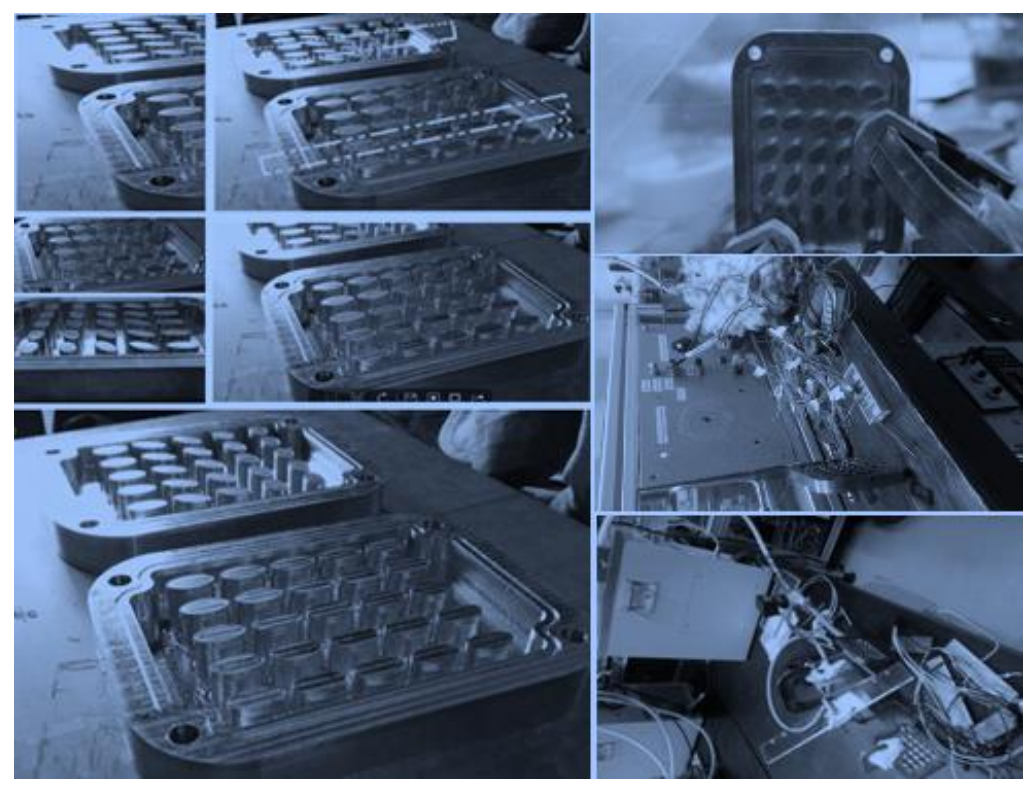

Figure 2.5. Production of MPFHS, Prototype (II)

The test section of heat sink prototype has been fabricated and fin shape effect on the fluid field has been conducted. The test section model (Fig 2.6) consists of pin fins, and the heating unit. They are assembled together with dimensions of $130 \mathrm{~mm}$ wide, $85 \mathrm{~mm}$ long and $30 \mathrm{~mm}$ height. The heating unit mainly consists of the heater and the thermal insulation. The heater output power is $869.56 \mathrm{~W}$ at $200.16 \mathrm{~V}$ and the measurement of current is $4.34 \mathrm{~A}$. The electrical power input is supplied to the heater by a DC source and controlled by a variac transformer to obtain constant heat flux along the base of the heat sink and measured by a digital wattmeter. The voltage settings are guided by the readings of thermocouples. Pump flow rate ranges from $0.0029 \mathrm{~kg} / \mathrm{s}$ up to $0.0320 \mathrm{~kg} / \mathrm{s}$ and calculations of power loss are given in table 2.1 and in the appendix. All sides of the heat sink are insulated except the top. The top surface is covered with a lexan sheet. To prevent water leak from the top, the lexan sheet is placed over a rubber packing. The lexan sheet is tightened to the heat sink with four bolts (Fig C.6). In order to obtain a clear picture of fluid flow, a particle image velocimetry (PIV) method 
of flow visualization is utilized. We have brightly illuminated the pin fin heat sink so the flow pattern could be visible and we used a PIV camera to view and visualized flow pattern as shown in Fig 2.7.a. and a photograph of the experiment is shown in Fig 2.7.b.

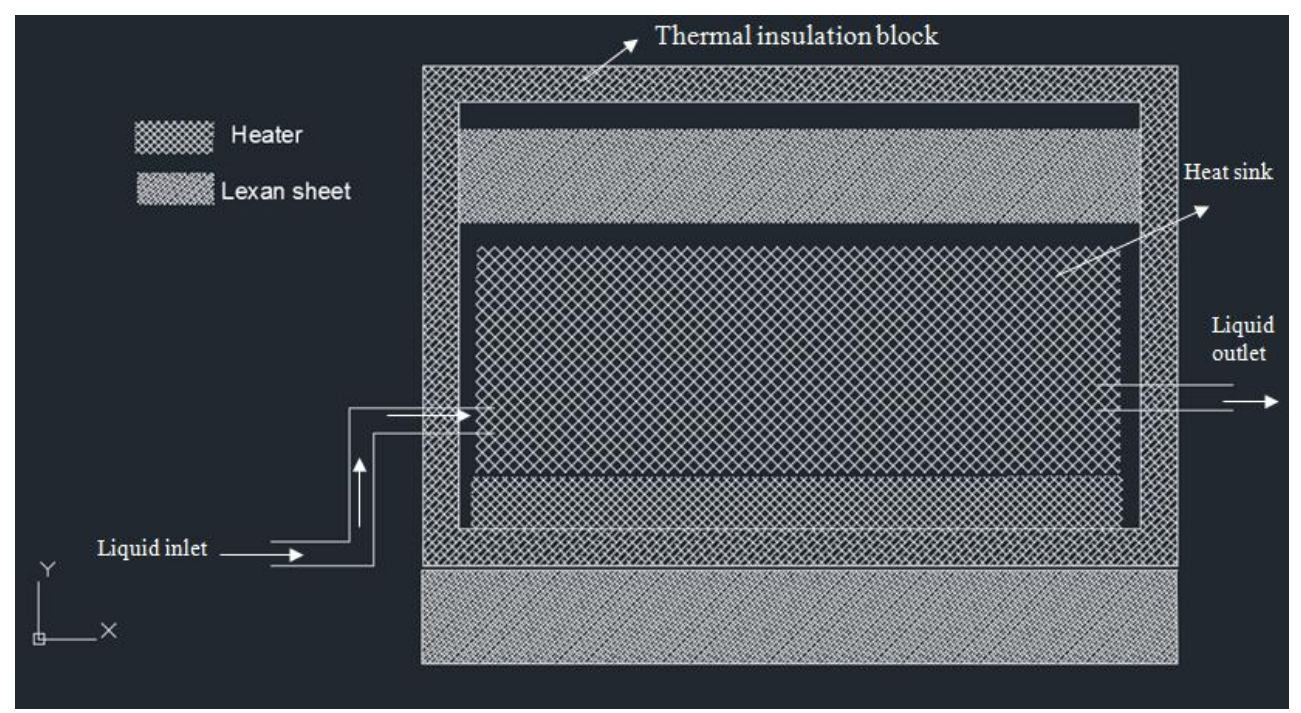

Figure 2.6. Schematic of test section

Table 2.1. Experimental Design Calculations

\begin{tabular}{|c|c|c|c|}
\hline \multicolumn{5}{|c|}{$\begin{array}{c}\text { Pump Flow Rate Range: }(0.0029 \mathrm{~kg} / \mathrm{s} \sim 0.320 \mathrm{~kg} / \mathrm{s}) \\
\text { Heat Sink Design: }(\text { Dia. } \uparrow, \text { Power } \uparrow)\end{array}$} \\
\hline velocity $(\mathrm{m} / \mathrm{s})$ & $\begin{array}{c}\text { Diameter of heat sink inlet } \\
(\mathrm{m})\end{array}$ & $\begin{array}{c}(\dot{m}) \text {, Mass flow rate } \\
(\mathrm{kg} / \mathrm{s})\end{array}$ & $\begin{array}{c}\text { Power loss } \\
(\text { Watts })\end{array}$ \\
\hline $\mathbf{2 . 0 0 0}$ & 0.0015 & 0.003464 & 80.7458 \\
\hline $\mathbf{2 . 2 5 0}$ & 0.0015 & 0.003897 & 90.8287 \\
\hline $\mathbf{2 . 5 0 0}$ & 0.0015 & 0.00433 & 100.921 \\
\hline $\mathbf{2 . 7 5 0}$ & 0.0015 & 0.004762 & 111.013 \\
\hline $\mathbf{2 . 7 5 0}$ & 0.0030 & 0.01905 & 444.052 \\
\hline $\mathbf{3 . 0 0 0}$ & 0.0030 & 0.020782 & 484.420 \\
\hline $\mathbf{3 . 2 5 0}$ & 0.0030 & 0.022513 & 524.788 \\
\hline $\mathbf{4 . 5 0 0}$ & 0.0030 & 0.031172 & 726.630 \\
\hline
\end{tabular}


At the channel inlet, where the temperature of the working fluids is originally uniform, that temperature is forced to change due to the development of the thermal boundary layers on the pins. We consider the water moves at a constant flow rate, and convection heat transfer occurs at the inner surface. The fluid is modeled as an ideal gas with negligible pressure variation. The axial variation of $T_{m}$, (Fig 2.8) can be readily determined. If $T_{s}>T_{m}$ heat is transferred to the fluid and $T_{m}$ increases with $x$; if $T_{s}<T_{m}$ the opposite is true. Although the surface perimeter $P$ sometime vary with $x$ along the length of the heat sink channel $(0<x<L)$ , mostly it is a constant (a heat sink of constant cross sectional area). Hence $P / \dot{m} C_{p}$ is constant.

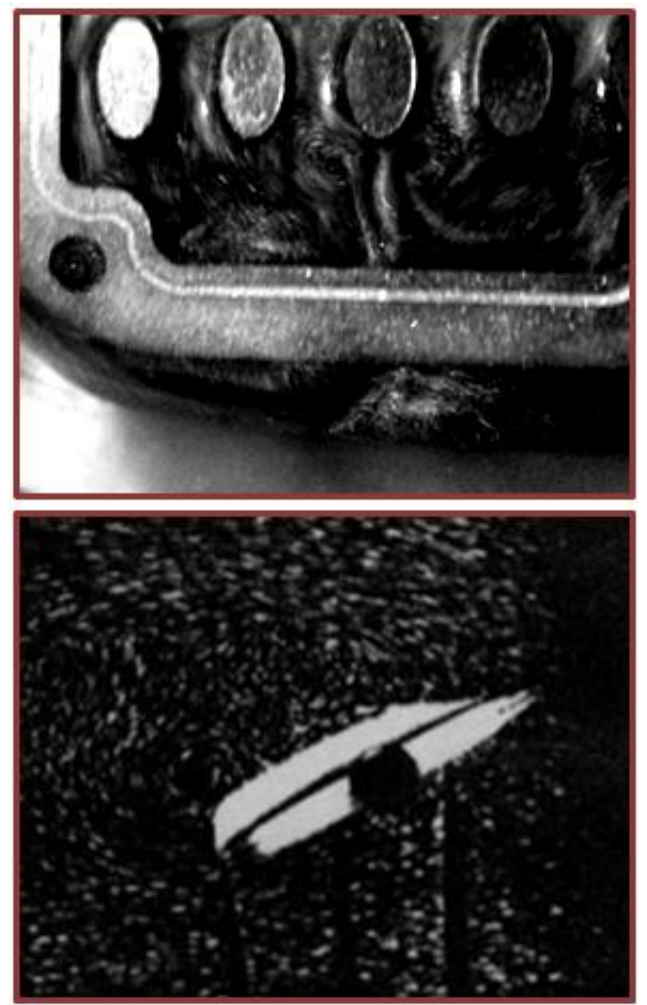

Figure 2.7.a. Visualization of the flow pattern: Top: current results, Bottom: results of Ohmi et al [34] 


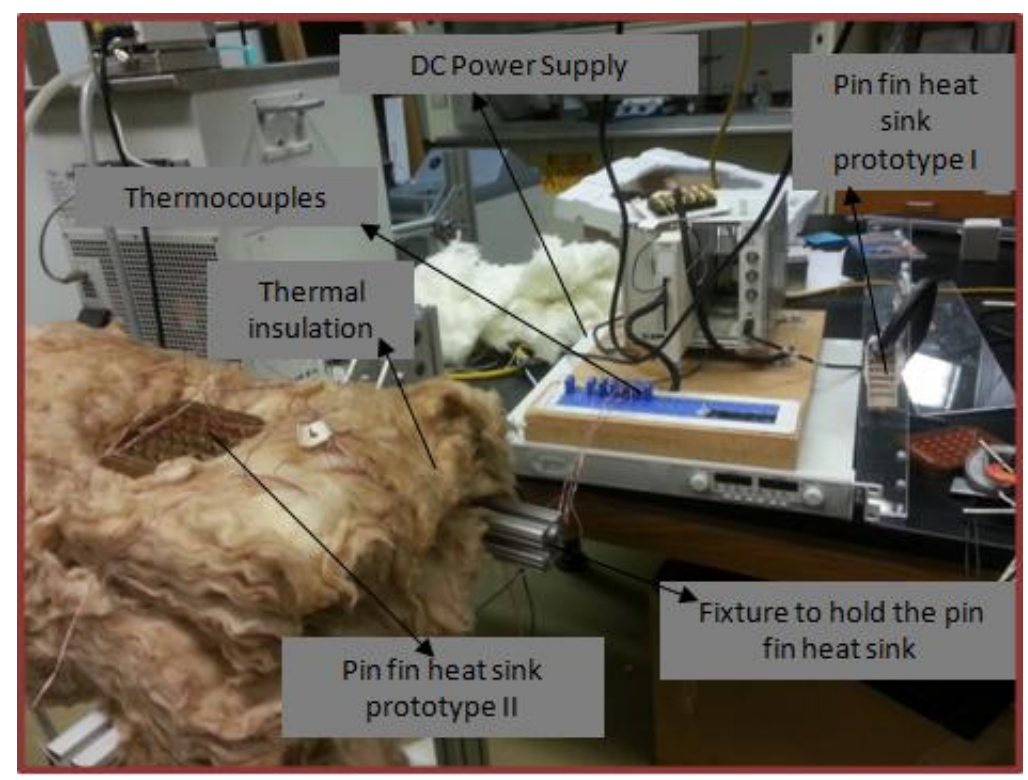

Figure 2.7.b. A photograph of the experiment

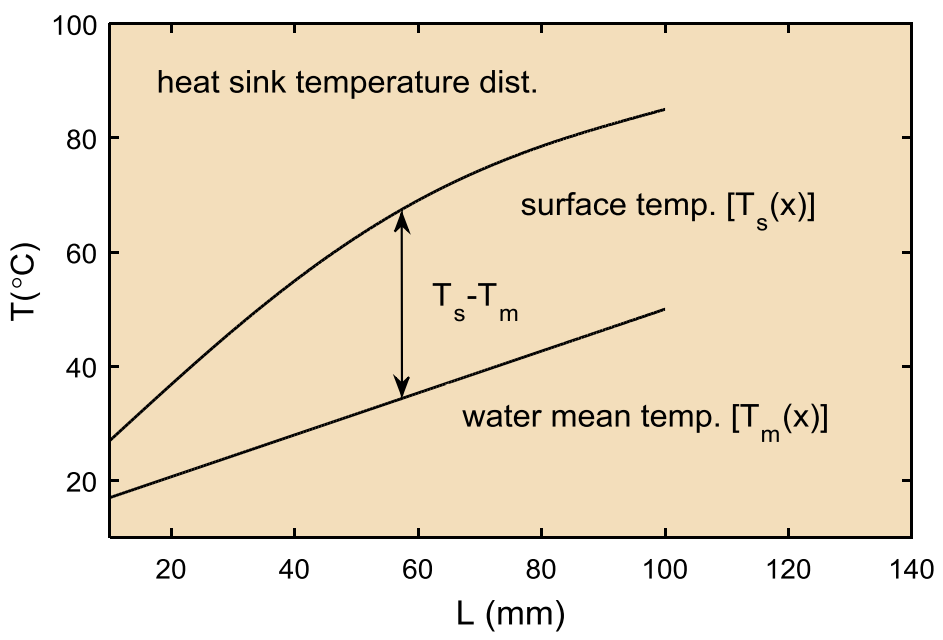

Figure 2.8. Fluid and surface axial temperature variations

It is important to note that the heat transfer coefficient $h$ varies with $x$ along the heat sink length $(0<x<L)$. Although $T_{s}$ can not be constant, $T_{m}$ must always vary with length of heat sink $(0<x<L)$ except when $\left(T_{s}=T_{m}\right)$. To determine the fluid temperature change, $\left(T_{m, e}-T_{m, i}\right)$ we integrate $d T_{m} / d x=q P / \dot{m} C_{p} \quad$ from $x=0$, and the following result, 
$T_{m}(x)=T_{m, i}+\left(q P / \dot{m} C_{p}\right) x$ is implemented. Accordingly, the fluid temperature varies linearly with $x$ from $x=0$ to $x=L$ along the heat sink. Furthermore, we see that the temperature difference $\left(T_{s}-T_{m}\right)$ to increase with position $(0<x<L)$. Hence, log mean temperature difference $\Delta T_{l m}$ increases with position along the heat sink. This difference is initially small (due to the small value of $h$ near the entrance) but there is an increase with increasing position along the heat $\operatorname{sink}(0<x<L)$ due to the increase in $h$, (Fig 2.9) that occurs as the boundary layer develops.

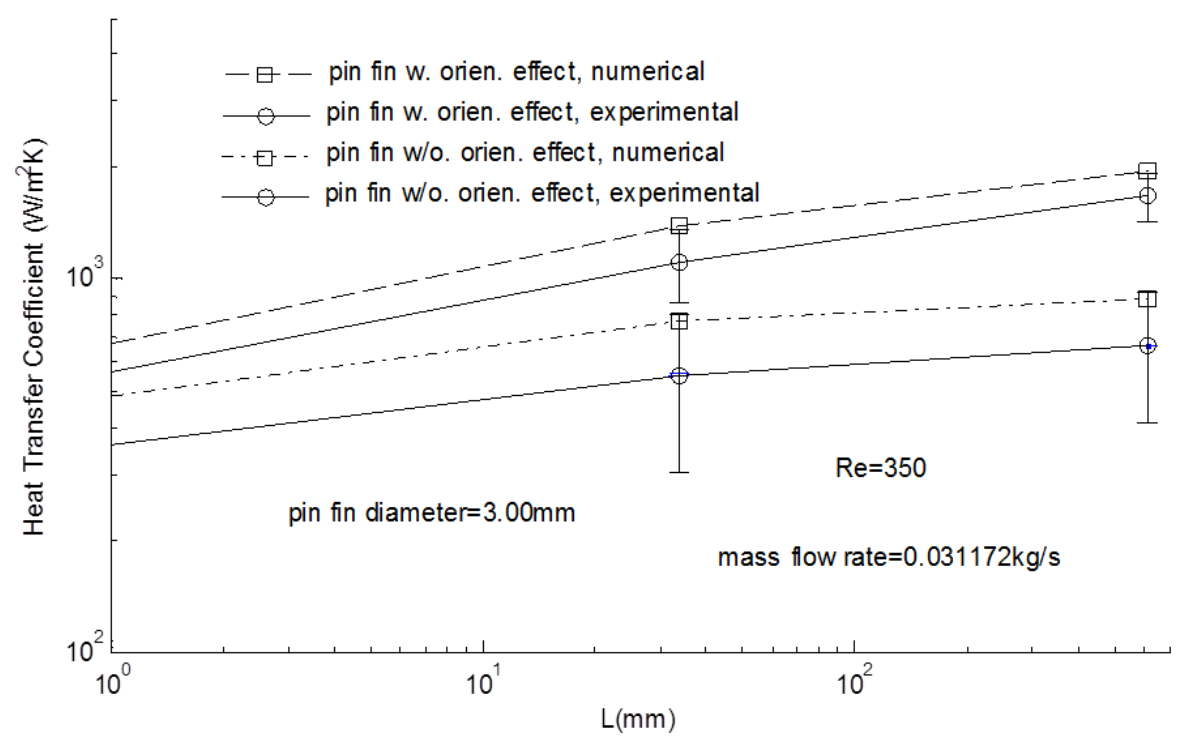

Figure 2.9. Heat transfer coefficient along the length of the pin fin heat sink

\subsection{RESULTS AND DISCUSSION}

The analysis was performed for the elliptical pin-fin heat sink, and the results are presented in this study. The temperature and velocity contours are explored first followed by an analysis of Euler and Nusselt numbers based on their response to the effect of the pins' angular orientation and the use of nanofluid as a coolant. Analyzing the temperature field in the coolant provides a good insight into heat transfer behavior. Calculations were performed using a wide 
range of Reynolds numbers and nanoparticle volume fractions with and without pin orientation cases. Figure 2.10 presents a comparison between contour of temperature distribution for both heat sinks with and without pin orientation for different Reynolds numbers in a plane which passes through half of the pins height. As expected the temperature is higher near the wall where the heat transfers to the coolant. The temperature distribution in the coolant is nonuniform and the orientation of pins intensifies this nonuniformity causing a significant difference between the average heat transfer from each pin in the system. In general, these differences are due to different flow behavior around pins, which is especially noticeable during the generation of larger circulation zones behind the pins with larger orientation angle. As seen, the flow and heat transfer field in the system with zero degree of orientation becomes fully developed after circulation around the third pin; however, for the system with angular orientation, that system will not become fully developed because of the complicated flow and heat transfer behavior around each pin. Furthermore, at the channel inlet, where the temperature of the working fluids is originally uniform, that temperature is forced to change due to the development of the thermal boundary layers on the pins. With an increasing Reynolds number, the thermal boundary layers on the pins decreases. Therefore, higher Reynolds numbers causes a larger heat transfer coefficient and consequently a larger Nusselt number. It can also be seen that the average bulk temperature of coolant decreases as the Reynolds number increases while the heat transfer rate between coolant and heated pin fins increases with increasing Reynolds number. These opposite trends can be explained as follows: convection heat transfer that occurs in the fluid region of MPFHS is comprised of two mechanisms:1) energy transfer due to the bulk motion of the coolant and 2) energy transfer due to diffusion in the coolant. At low Reynolds number, the coolant's mean velocity is low, and it has more time to absorb and spread heat; therefore, 
diffusive heat transfer is the dominant player, causing the coolant to obtain a higher bulk temperature. On the other hand, as the Reynolds number increases, the mean fluid velocity increases, and forced convection plays a higher role in the heat transfer, there by transferring more heat without much increase in temperature. Similar behaviors can be seen for cases where nanofluid is the coolant.

\section{Re $=$ Low}

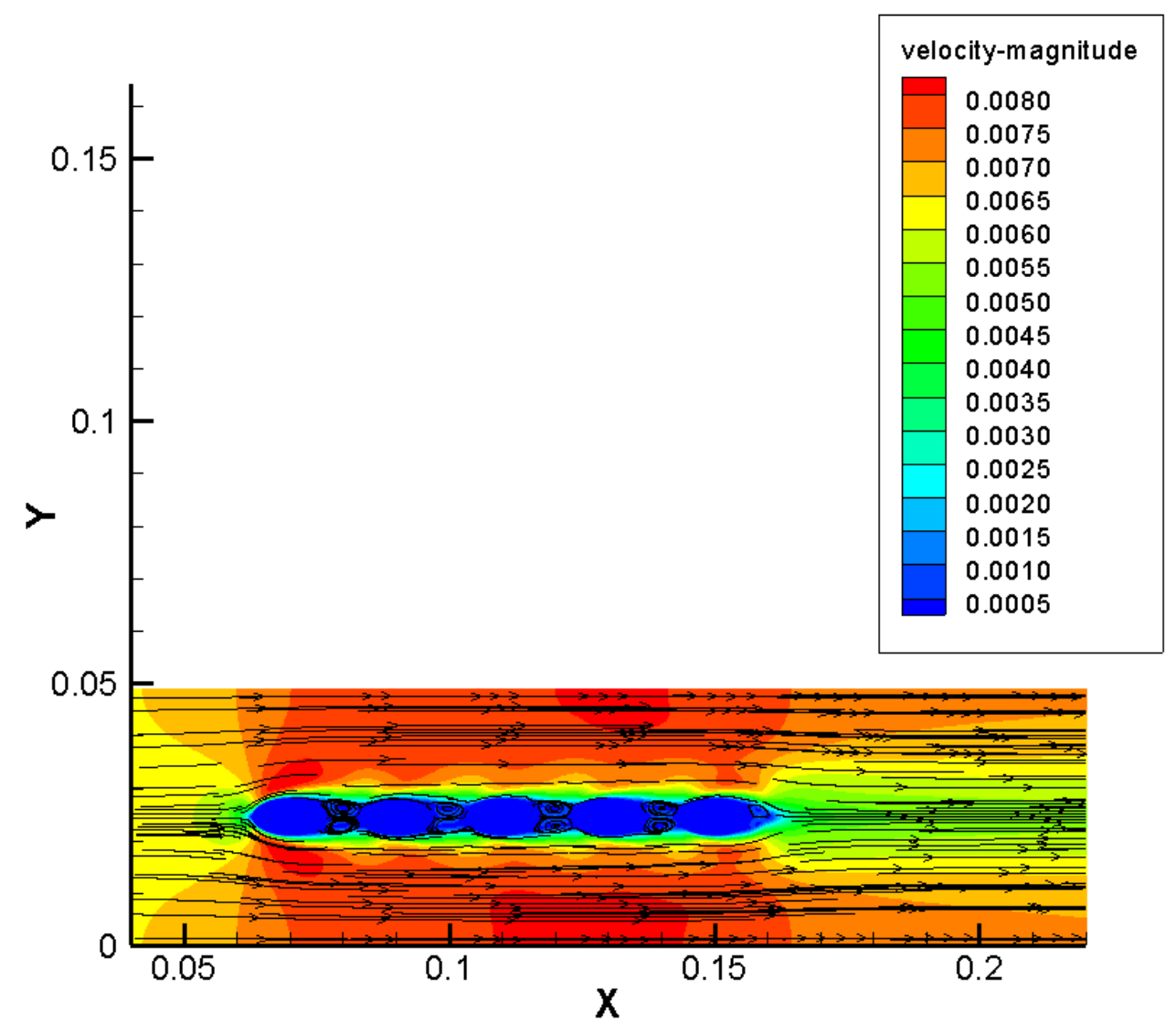



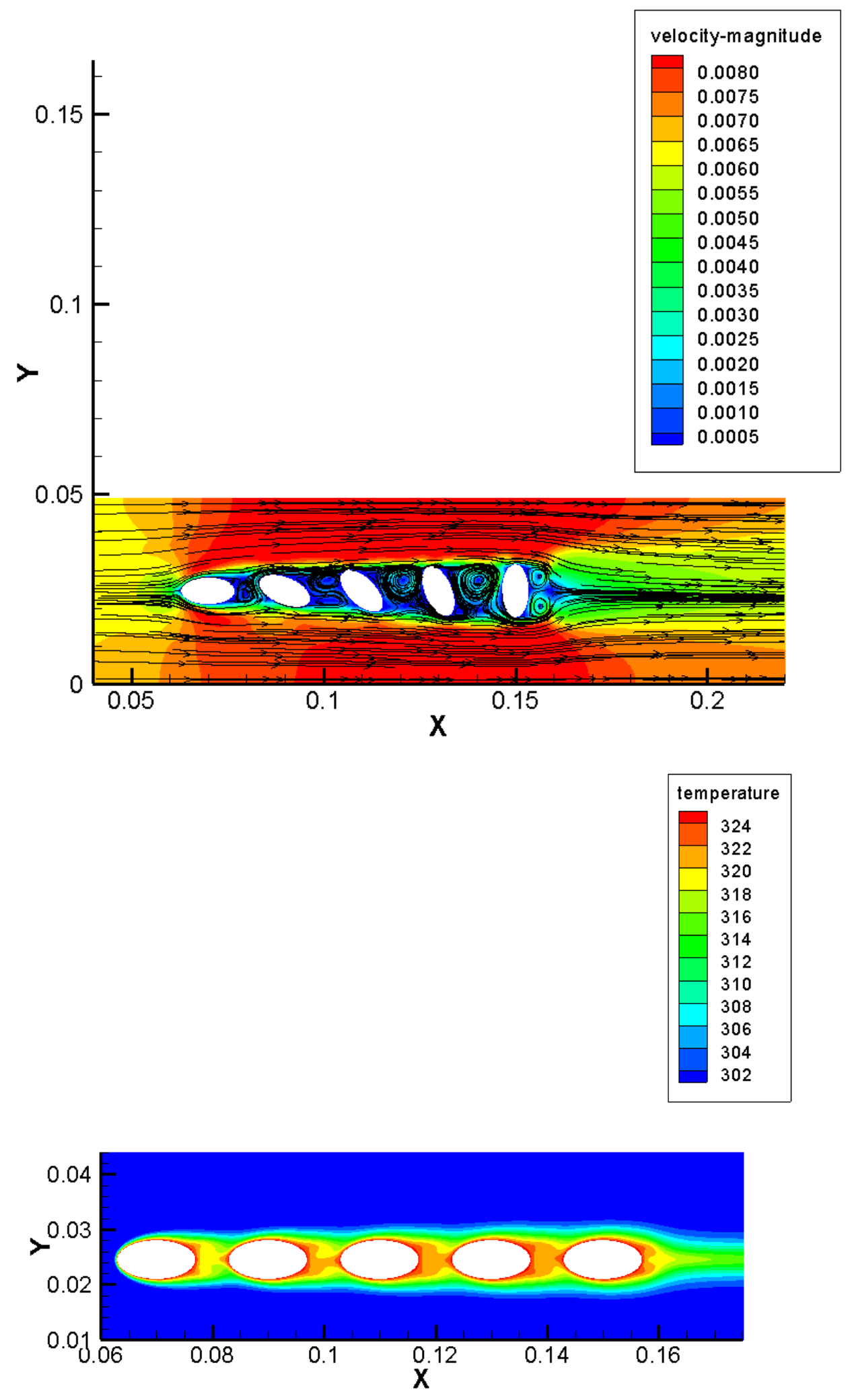

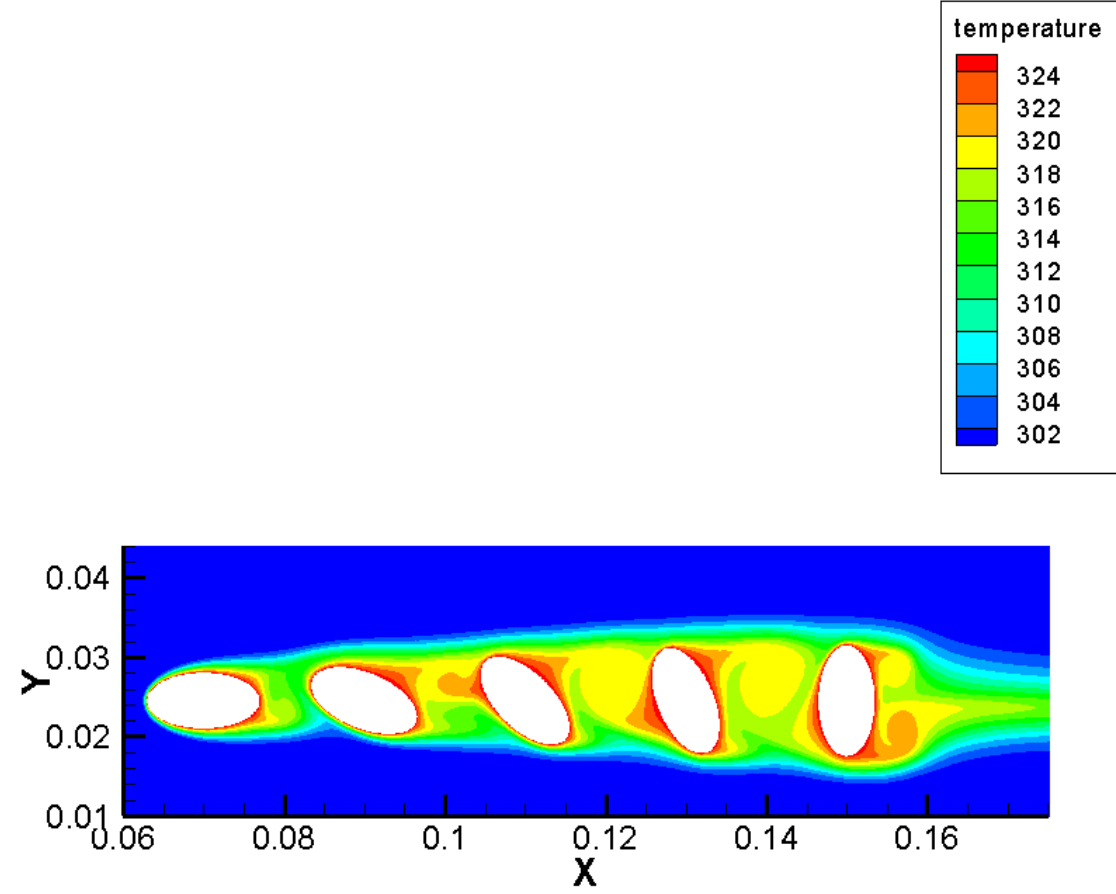

\section{$\mathbf{R e}=$ Medium}

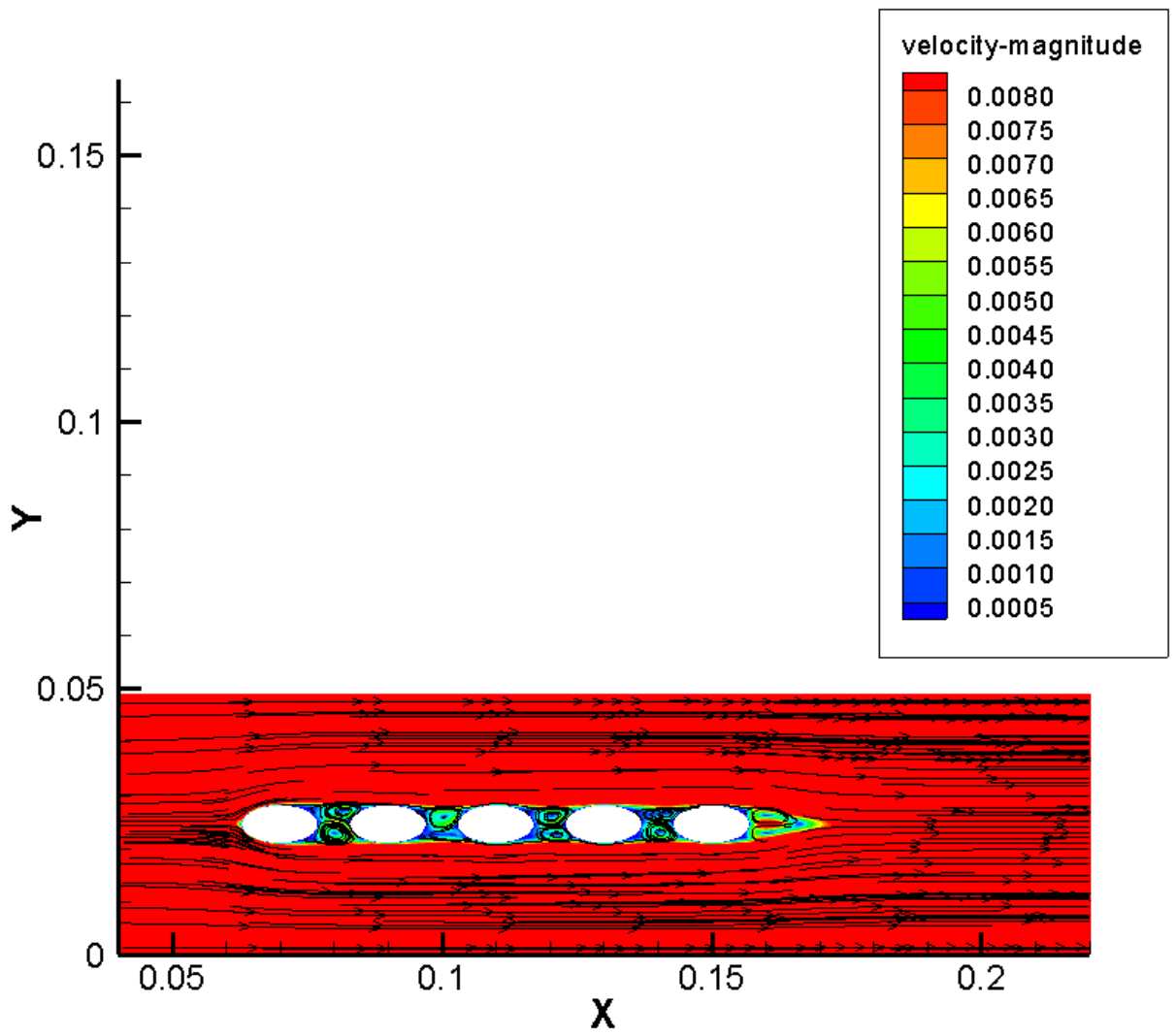




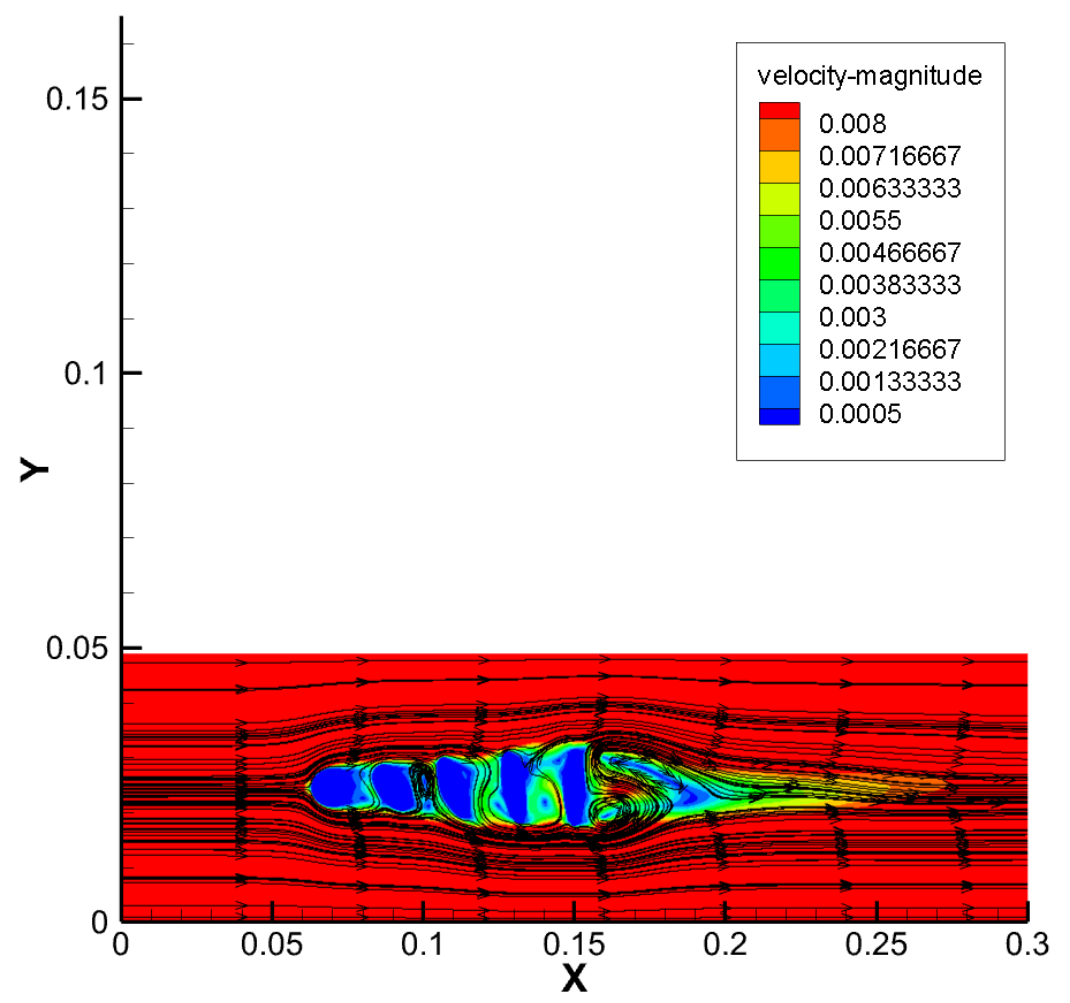

\begin{tabular}{||l|}
\hline \multicolumn{2}{|c|}{ temperature } \\
\hline \\
324 \\
322 \\
320 \\
\hline 318 \\
316 \\
314 \\
312 \\
310 \\
308 \\
306 \\
304 \\
302
\end{tabular}

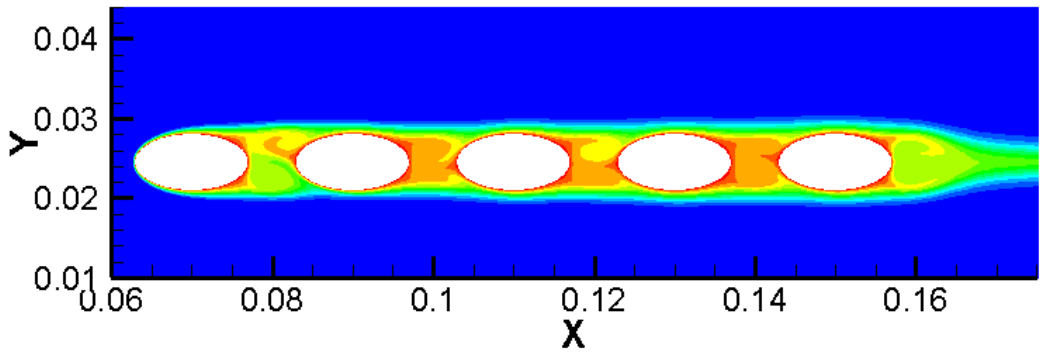




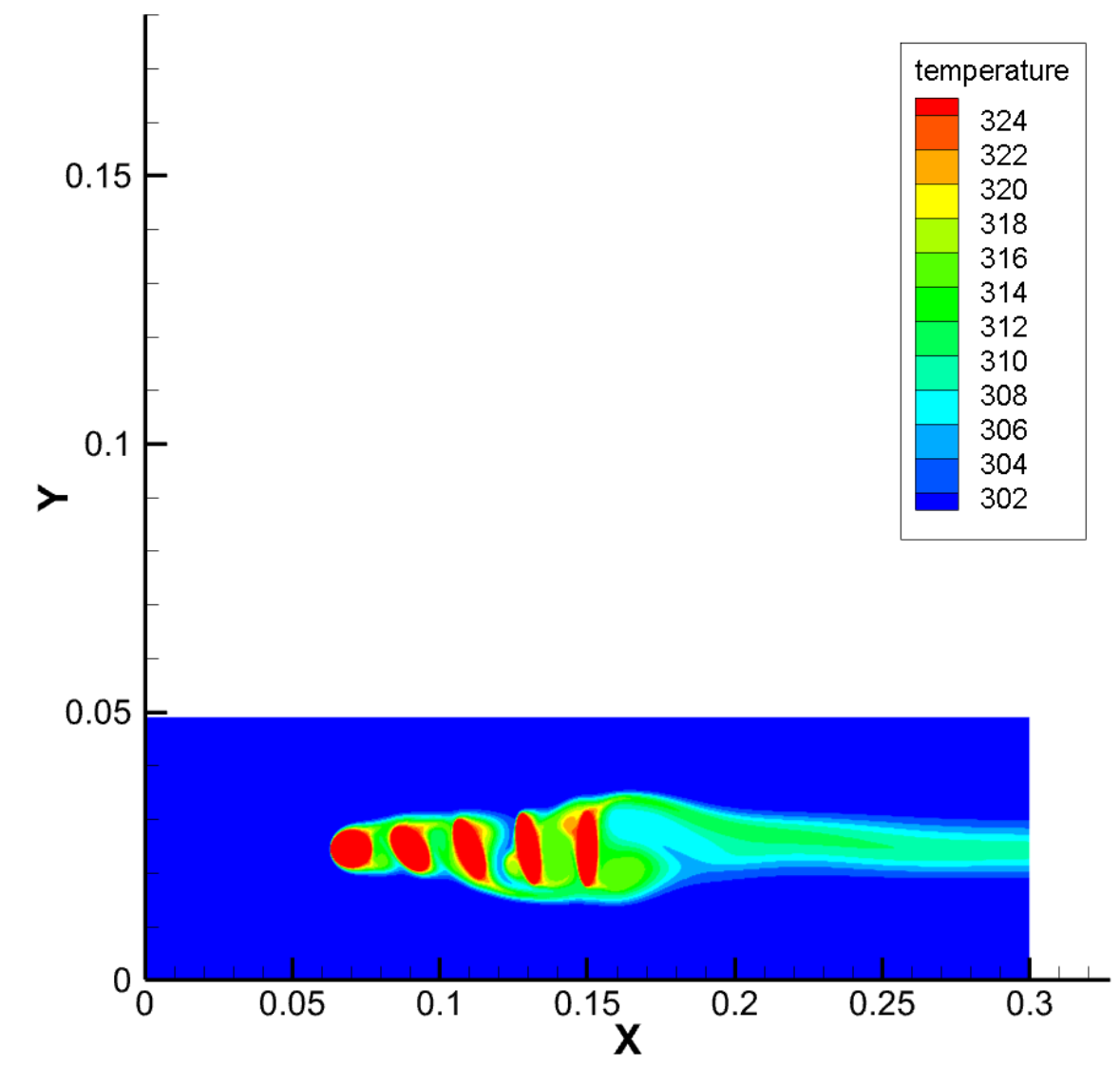

Re $=$ High

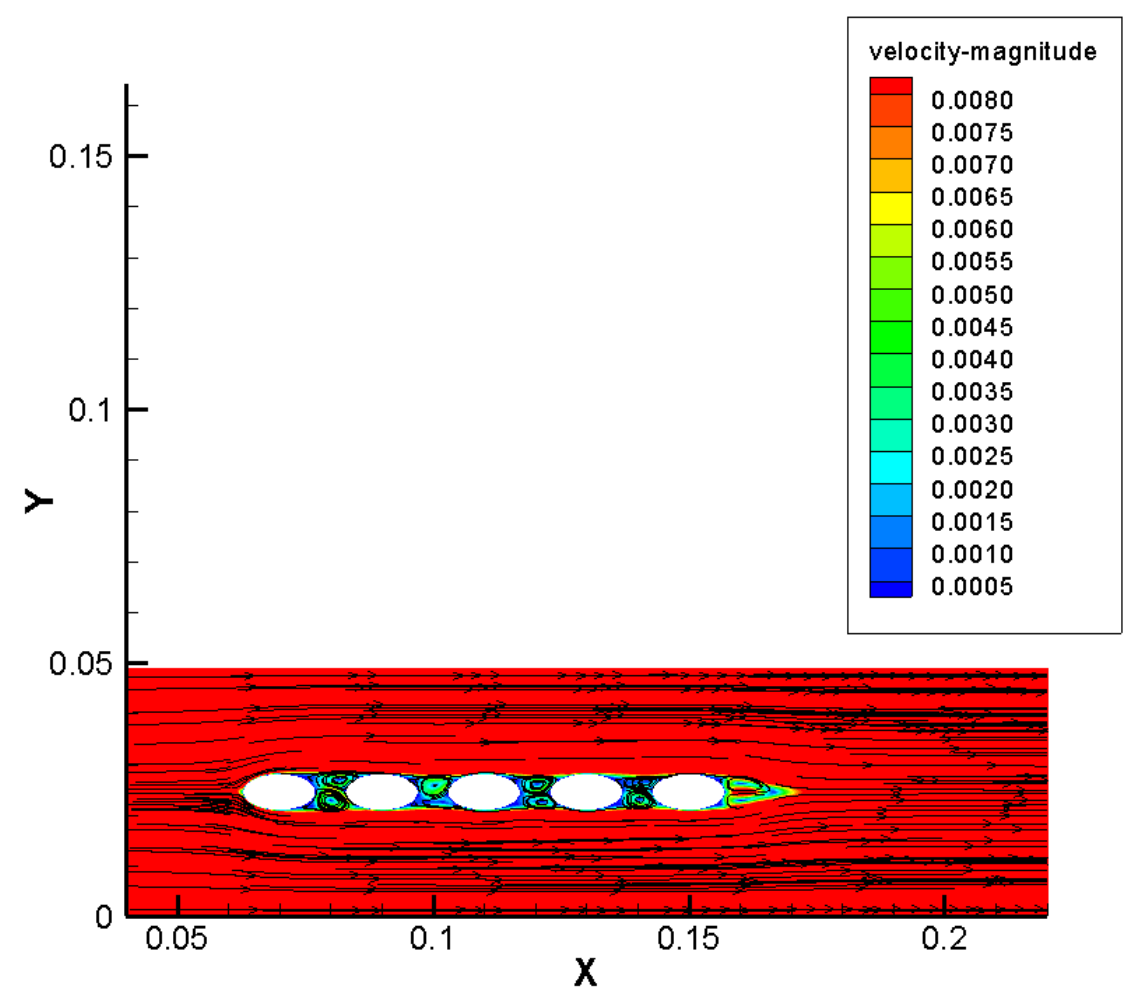



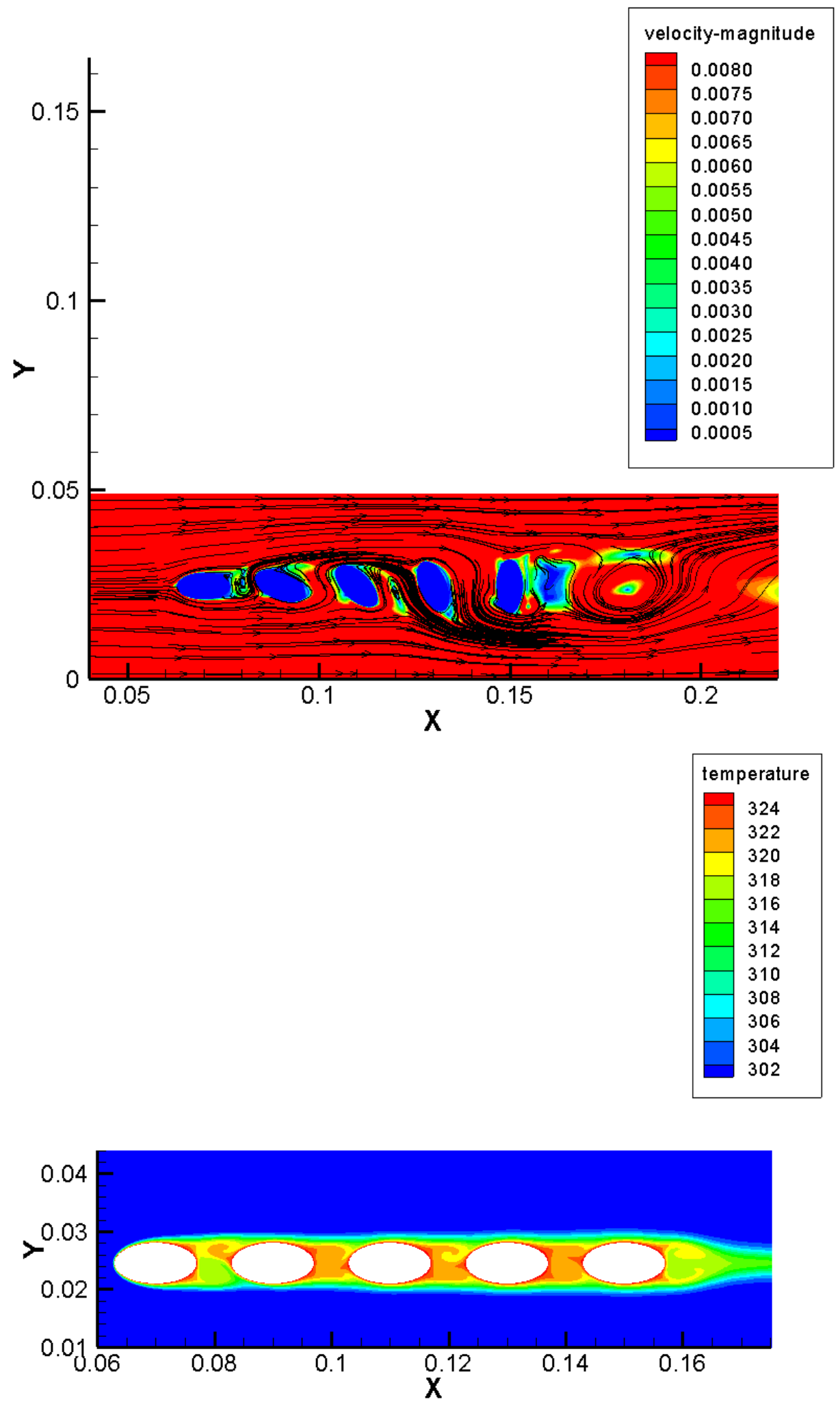


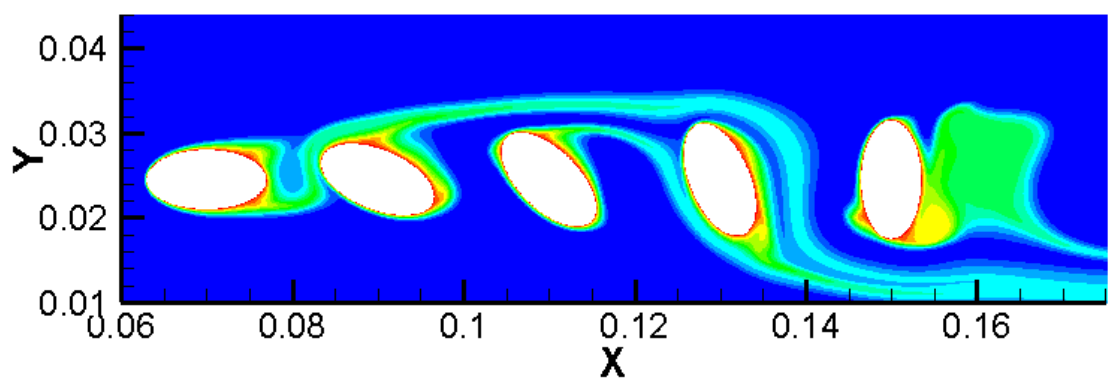

Figure 2.10. Temperature and velocity distributions around two different configurations of elliptical pin fins at three different Reynolds numbers with nanofluid as coolant.

The average Nusselt number for each individual pin in the system as well as the overall Nusslet number for heat sink (averaged for all pins) are shown in figure 2.11 (a-f).It can be seen that at a given Reynolds number, both the average Nusselt number of individual pins and the total Nusselt number increases with increasing nanoparticle volume fraction. Therefore, higher volume fraction results in more effective cooling. The influence of nanoparticles elucidates two opposing effects on the heat transfer in the heat sink: 1) a favorable effect that is driven by the presence of high thermal conductivity of nanoparticles and 2) an undesirable effect promoted by high level of viscosity experienced at high volume fractions of nanoparticles. In other words, the addition of nanoparticles to the base fluid enhances the thermal conduction and consequently the convective heat transfer coefficient; hence, as the particle volume fraction increases so does the Nusselt number enhancement. An interesting result (figure 2.12) is the effect of orientation of 
pins on the average heat transfer of each individual pin as well as the total heat transfer of the system. As seen, changing the orientation of pins causes the Nusselt number for each individual pin to increase, and the heat transfer enhancement depends on the degree of orientation. The lowest enhancement occurs for the first pin because of similarity of flow and heat transfer around the pin in the case without pin orientation. However, depending on the flow conditions ( Re and orientation angle), the highest enhancement occurs in third, fourth or fifth pin.

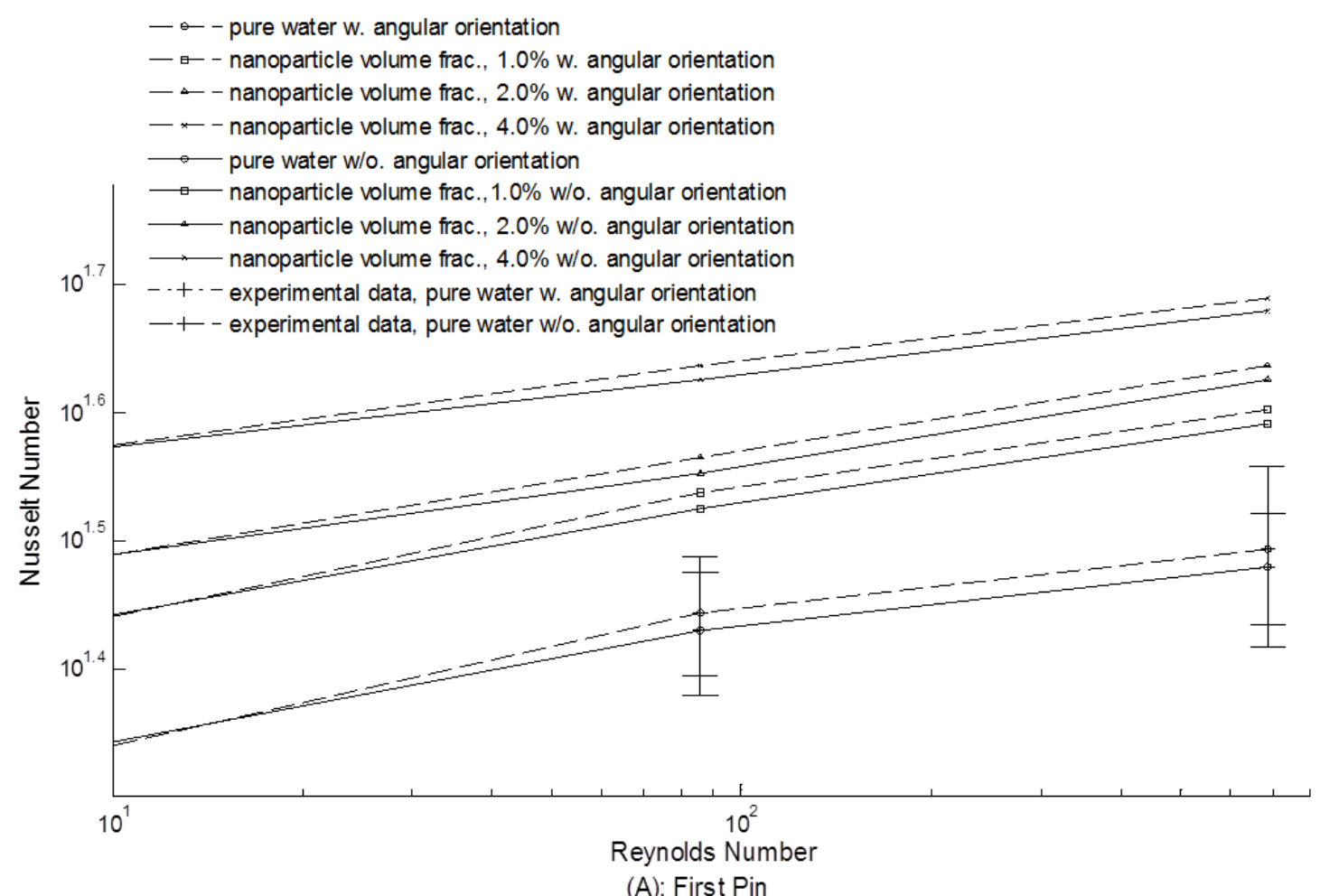




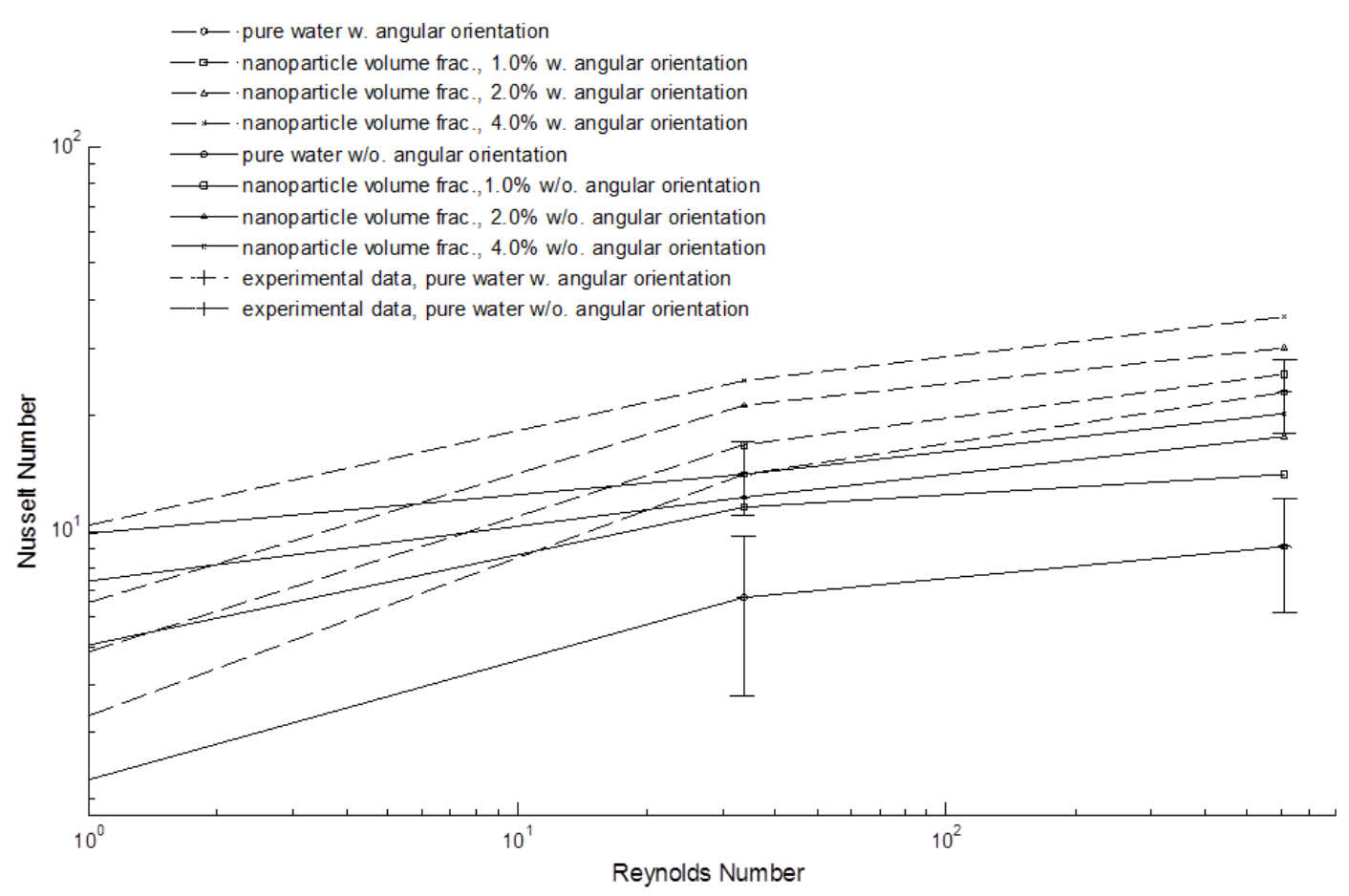

(B): Second Pin

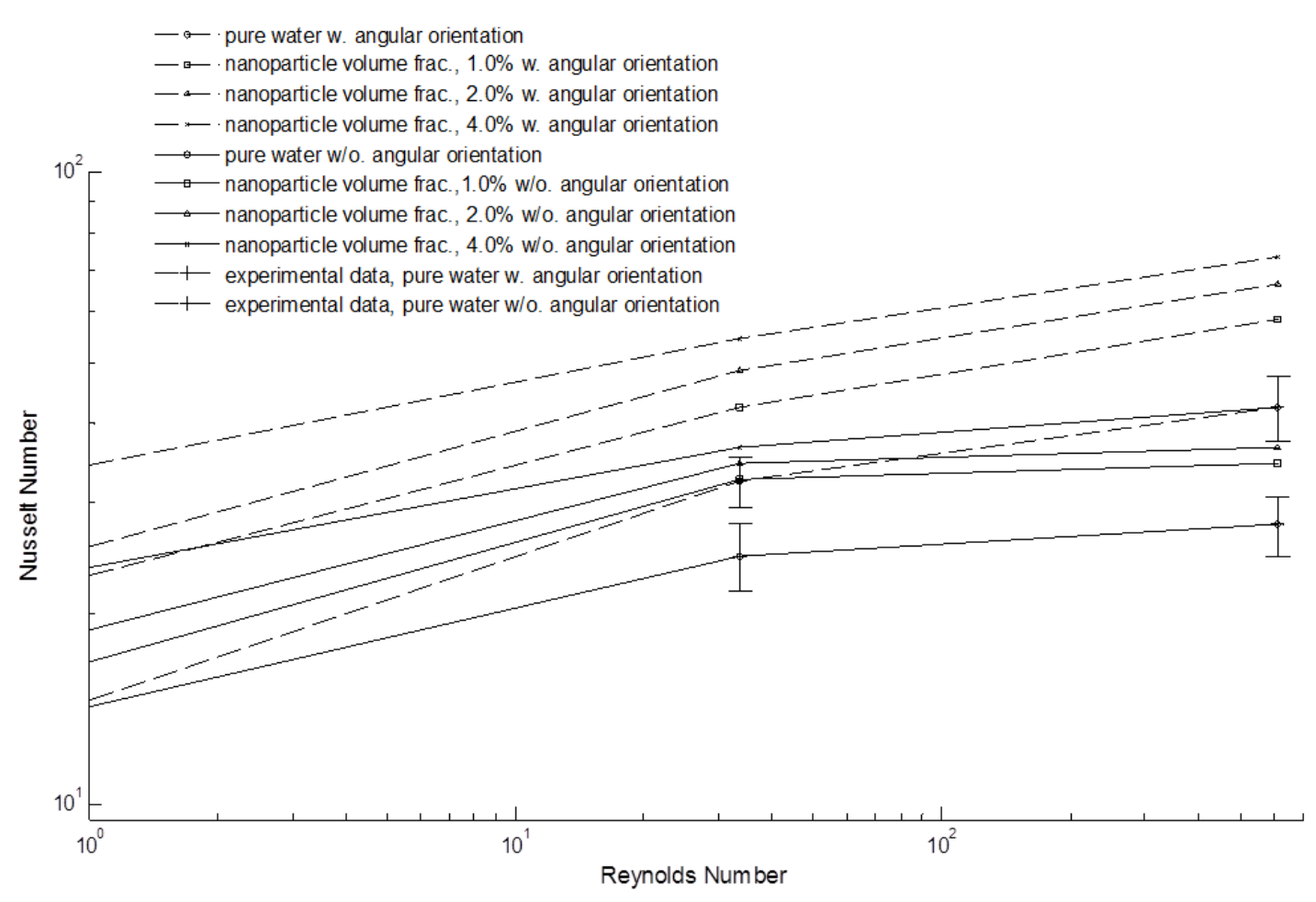

(C): Third Pin 

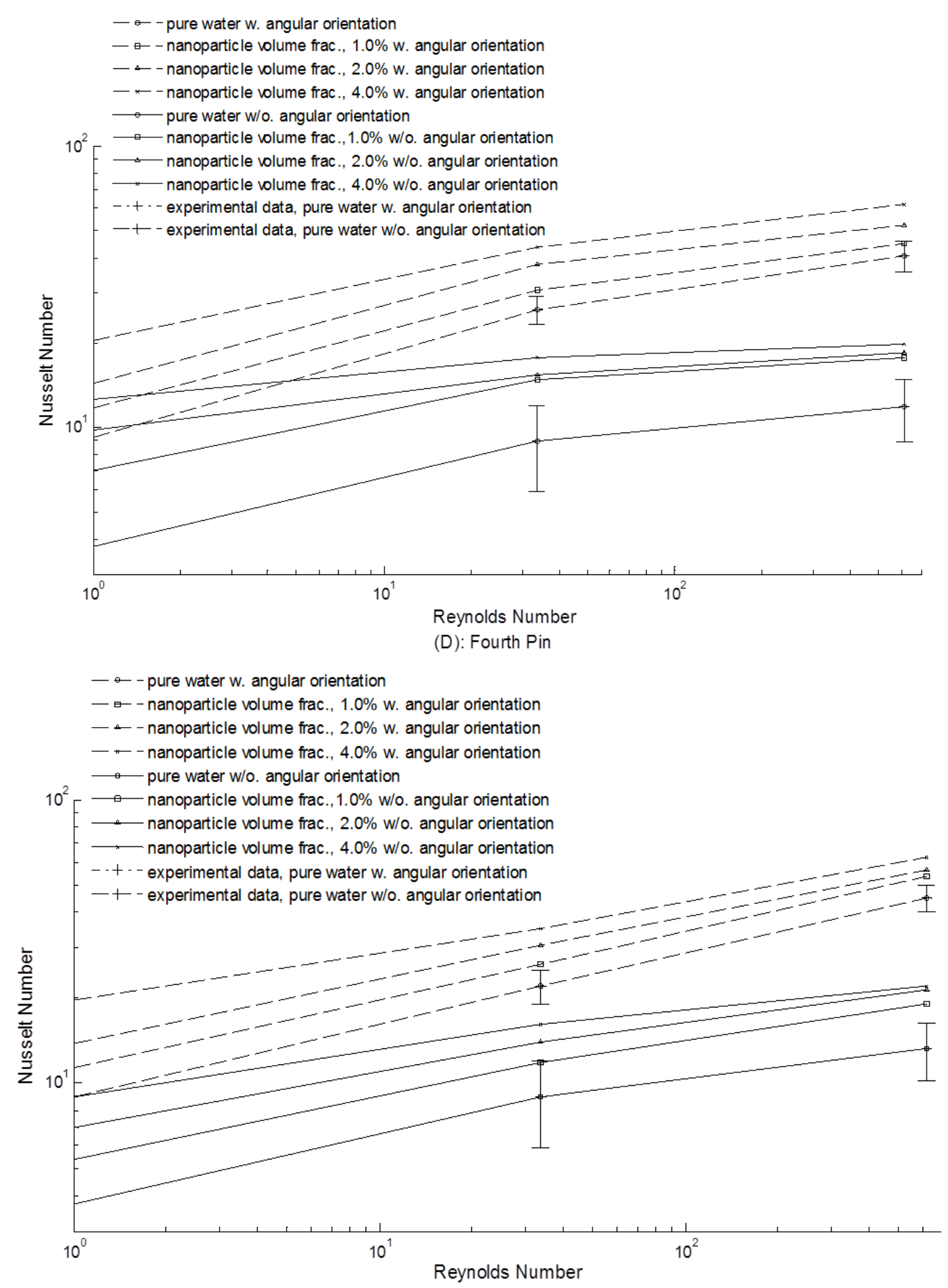

(E): Fifth Pin 


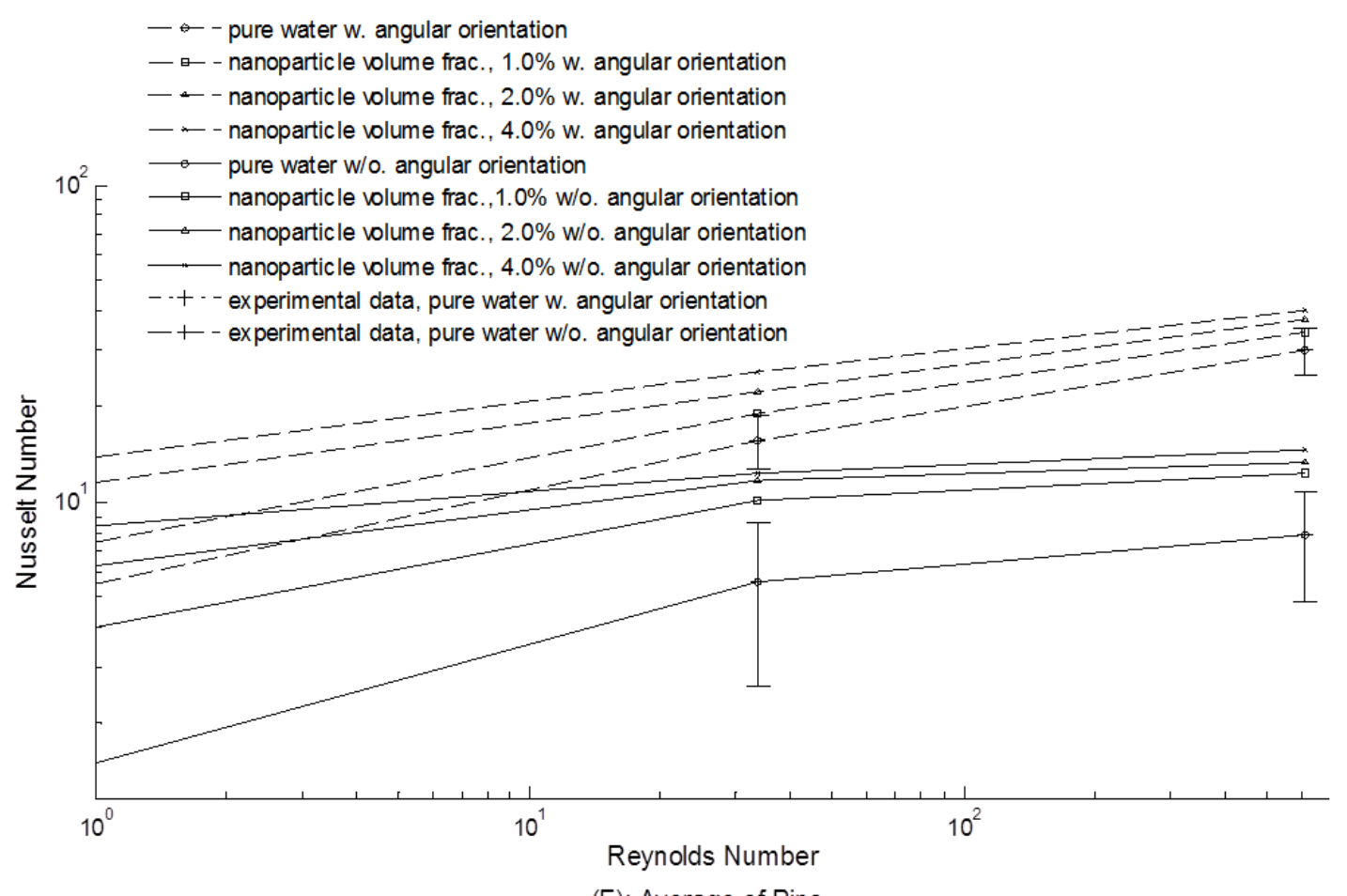

$(F)$ : Average of Pins

Figure 2.11. Effect of pin orientation and nanofluid volume fraction on Nusselt number at various Reynolds numbers, (a) Nusselt number of first pin, (b) Nusselt number of second pin, (c) Nusselt number of third pin, (d) Nusselt number of forth pin, (e) Nusselt number of fifth pin, (f) overall Nusselt number of heat sink

Table 2.2. Comparison between results of Kosar and Peles [33] and current results

\begin{tabular}{|c|c|c|c|}
\hline \multicolumn{3}{|c|}{ Nusselt Number } \\
\hline $\mathbf{R e}$ & Ref. [33] & Current & Difference (\%) \\
\hline $\mathbf{1 4 . 2 0}$ & 0.80 & 0.82 & 2.5 \\
\hline $\mathbf{3 6 . 4 4}$ & 2.30 & 2.41 & 4.78 \\
\hline $\mathbf{5 5 . 3 8}$ & 4.72 & 4.97 & 5.29 \\
\hline $\mathbf{1 1 2 . 2}$ & 7.14 & 7.64 & 7.01 \\
\hline
\end{tabular}


Table 2.3. Comparison between results of Yang et al. [32] and current results

\begin{tabular}{|c|c|c|c|c|c|c|c|}
\hline & \multicolumn{4}{|c|}{$h\left(W / \mathrm{m}^{2} K\right)$} & \multicolumn{3}{|c|}{$\Delta p(p a)$} \\
\hline & $\begin{array}{c}\text { velocity } \\
(\mathrm{m} / \mathrm{s})\end{array}$ & $\begin{array}{l}\text { Ref. } \\
{[32]}\end{array}$ & Current & $\begin{array}{l}\text { Diff. } \\
(\%)\end{array}$ & $\begin{array}{l}\text { Ref. } \\
\text { [32] }\end{array}$ & Current & $\begin{array}{l}\text { Diff } \\
(\%)\end{array}$ \\
\hline \multirow{3}{*}{$\begin{array}{c}\text { Inline } \\
\text { Configuration }\end{array}$} & 1 & 30.1 & 28.78 & 4.36 & 2.14 & 2.08 & 2.61 \\
\hline & 2 & 57.29 & 53.57 & 6.48 & 7.28 & 7.01 & 3.84 \\
\hline & 3 & 78.15 & 71.35 & 8.69 & 13.39 & 12.58 & 5.98 \\
\hline \multirow{3}{*}{$\begin{array}{c}\text { Staggered } \\
\text { Configuration }\end{array}$} & 1 & 42.85 & 40.66 & 5.11 & 0.97 & 0.94 & 2.98 \\
\hline & 2 & 72.64 & 66.9 & 7.89 & 4.02 & 3.85 & 4.11 \\
\hline & 3 & 93.41 & 84.55 & 9.48 & 8.11 & 7.59 & 6.34 \\
\hline
\end{tabular}

In addition to the advantage of increasing the system's thermal performance by adding nanoparticles to the base fluid and changing the orientation of pins, a drawback is also associated with increase of pressure drop relating to volume fraction and pins' angular orientation. Figure 2.12 depicts that changing the angular orientation of pins and increasing volume fraction of nanoparticles(which is responsible for larger heat transfer performance) will lead to a higher Euler number and consequently a pressure drop in the system. The sensitivity of the Euler number to volume fraction of nanoparticles is related to the increased viscosity when the nanoparticles attain the higher volume fractions. These high values of volume fractions lead the fluid to become more viscous, which causes more pressure drop and correspondingly enhancement in the Euler number. As expected, an increase in volume fraction causes the Euler number to increase, but accordingly, the increase is not very significant because of the small increase in viscosity when using nanofluids, which will not cause a noticeable penalty on pressure drop. However, for cases with angular orientation, the pressure drop is significant, and it increases with an increasing Reynolds number. 


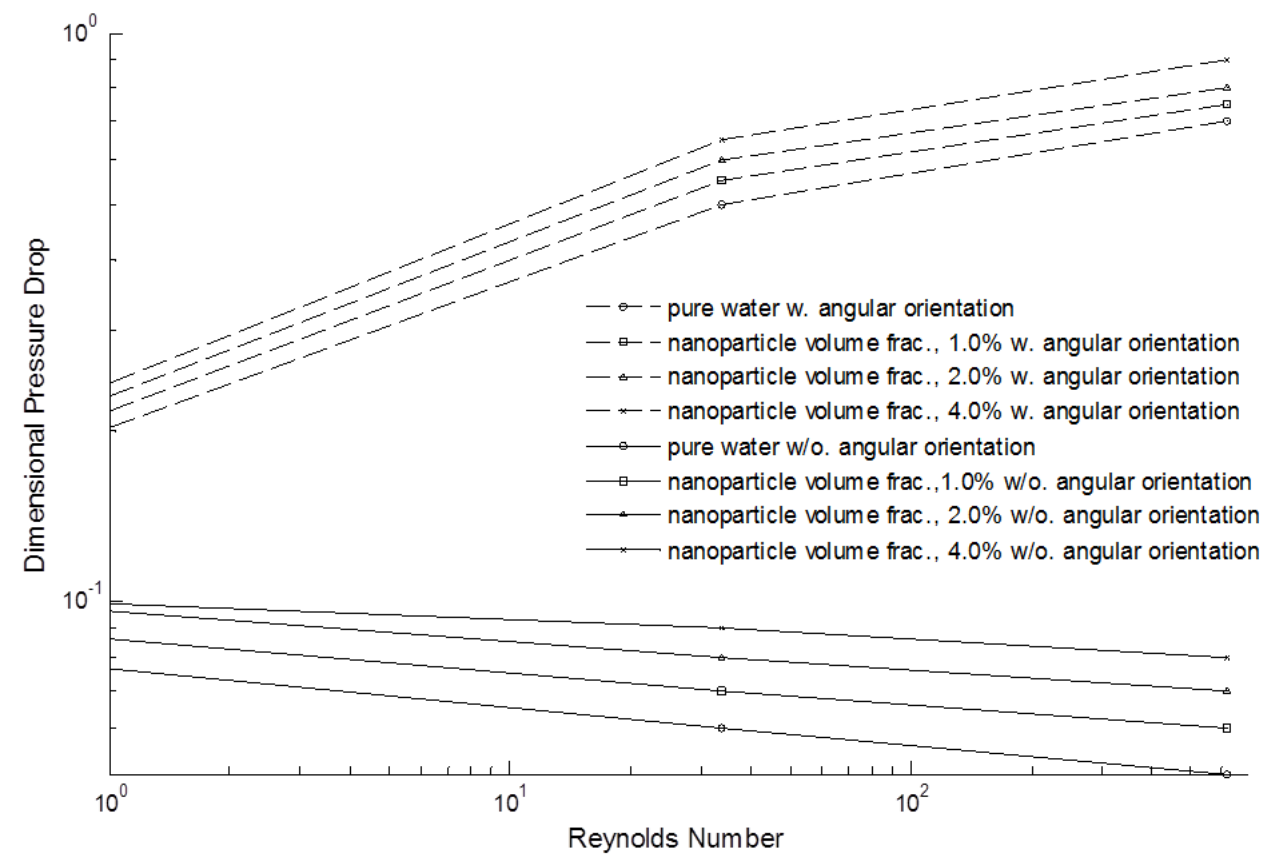

Figure 2.12. Effect of pin orientation and nanofluid volume fraction on Euler number at various

Reynolds numbers

\subsection{SUMMARY}

The forced convective heat transfer on nanofluids in an elliptical pin-fin heat sink of two different pin orientations was numerically studied by using a finite volume method. With increasing Reynolds number, the recirculation zones behind the pins increased. There were more recirculation zones for the pins with different angular orientations than for pins with the same angular orientation. It was observed that the Nusselt number for the pins with different angular orientations was higher than that for pins with the same angular orientation. The results show that with increasing volume fraction of nanoparticles and angular orientation of pins for a given Reynolds number, Euler and Nusselt numbers as well as overall heat transfer efficiency increase. Experimental investigation for the pin fin design provided insight including the existence of an optimum fin configuration. The pin fins with different orientation angle outperformed in comparison to pin fins with same orientation angle. 


\section{CHAPTER THREE: UTILIZATION OF THIN FILM EVAPORATION IN A TWO- PHASE FLOW HEAT EXCHANGER}

\subsection{INTRODUCTION}

Pin fin heat sinks and heat exchangers are classified as either single-phase or two-phase according to whether fluid superheats inside the microchannels. The primary parameters that determine the single-phase and two-phase operating regimes are the heat flux and the mass flow rate. For uniform heat flux, the fluid may maintain its liquid state throughout the microchannels. For higher heat flux and mass flow rate, the fluid flowing inside the microchannel superheats, resulting in a two-phase heat exchanger.

The temperature is higher near the wall of the two-phase heat exchanger where the heat transfers to the coolant and the heat generated must be dissipated in order to keep temperature distribution uniformity. Based on heat flux, the liquid film around thin film region is often divided into three regions, namely, the non-evaporating film region, evaporating film region, and intrinsic meniscus region as shown in figure 3.1.

Thin-film evaporation plays an important role in this two-phase system. A thin liquid film is formed, confined inside the elliptic pin fin heat sink and governed by evaporating liquid phase.When thin-film evaporation occurs, most of the heat transfers through a narrow area between a non-evaporation region and an intrinsic meniscus region. Because thin-film evaporation occurs in a small region, increasing the thin-film region and maintaining its stability is very important. 


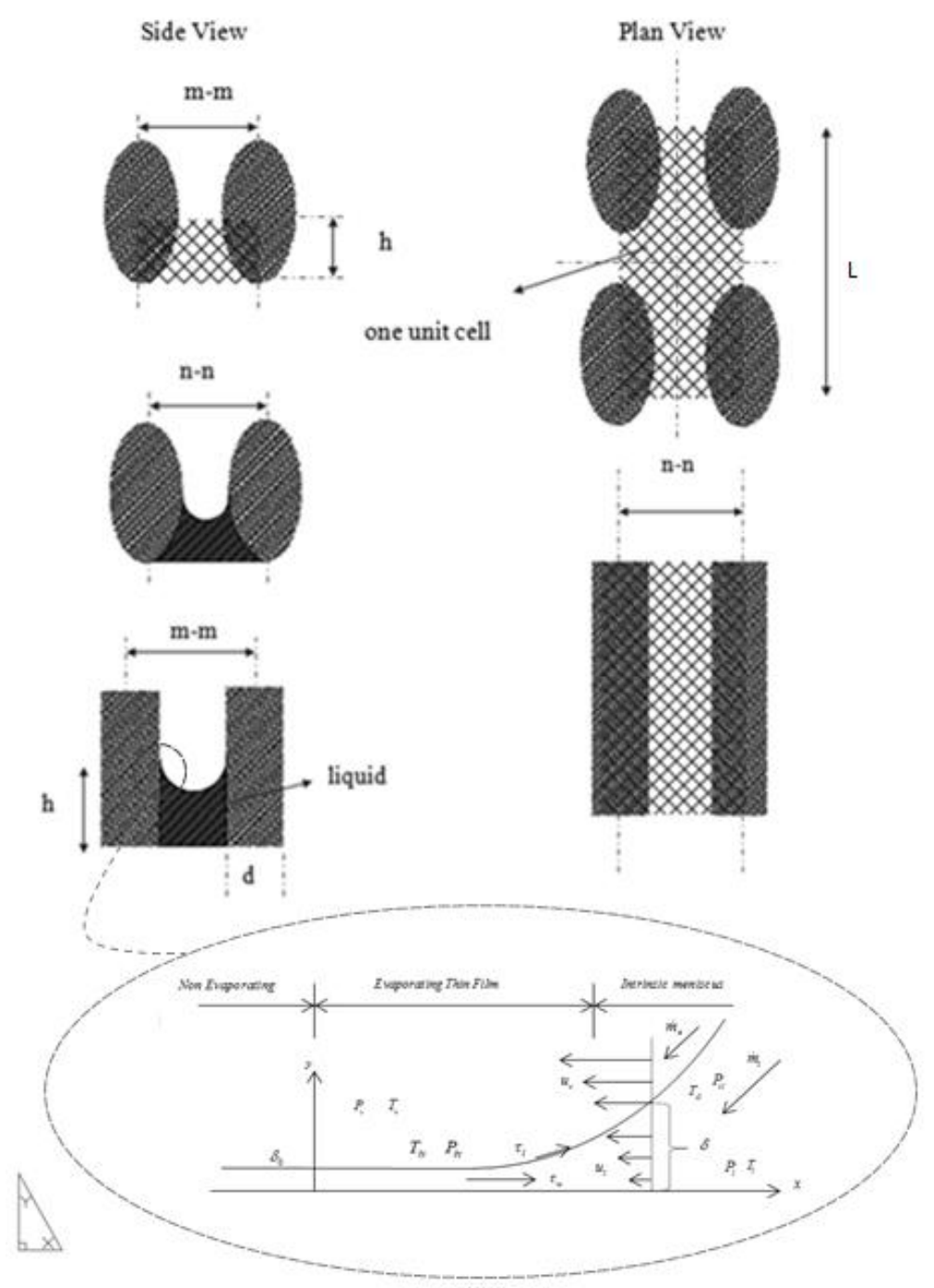

Figure 3.1. Schematic of thin film evaporation

The flow and heat transfer in the evaporating thin film region, which are driven by the superheat, capillary and disjoining pressure gradients, are the strongest among the three regions due to the relatively small thermal resistance across the film. Intermolecular forces between liquid thin film and wall are characterized by disjoining pressure. The disjoining pressure controls the wettability and stability of liquid thin film formed on the wall. The non evaporating region has no evaporation due to the strong disjoining pressure even though the liquid-vapor interface is usually superheated to a wall temperature. Thus, the disjoining pressure plays key role and affect the interface temperature and heat flux through the thin film. 
Many researchers, both experimentally and theoretically, studied different systems to understand the details of interfacial phenomena by studying the roles of the disjoining pressure [35-39] and surface tension and its gradient [35-44] on the wetting phenomena [37], Marangoni shear in the contact line region of evaporating meniscus [35-37], instabilities [35-38], fluid flow, and microscale phase change phenomena [35-44]. Investigations have conducted to understand mechanisms of fluid flow coupled with evaporating heat transfer in thin-film region. For example, Ma and Peterson [39, 40] studied the thin-film profile, heat transfer coefficient, and temperature variation along the axial direction of a triangular groove. Wayner et al. [41, 42], extended the Clausius-Clapeyron equation and the approximation has been widely used to relate the liquid- vapor interfacial temperature and pressure differences to the evaporative heat flux. They showed that the adsorbed film thickness decreases from when the wall superheat increases. Park et al. $[43,44]$ showed that the vapor pressure gradients significantly affect the thin film profile. They concluded that as the heat flux increases, the length of thin film region and the film thickness decrease and the local evaporative mass flux increases linearly.

In this chapter, the Young-Laplace equation and the Clausius-Clapeyron equation predicting fluid flow and heat transfer for evaporating thin film region is presented and non dimensional analytical investigation is performed. Investigations of fluid flow effects are performed in thin-film region which include the disjoining pressure and surface tension and its gradient, Marangoni shear in the contact line region, and phase change phenomena. The superheat effects on film thickness are also performed.

\subsection{THEORETICAL ANALYSIS}

The heat input is assumed to travel through the porous medium. The liquid is continuously filled up from the intrinsic meniscus region into the evaporating thin film region to 
replace the evaporation mass loss. When the film thickness approaches the adsorbed film thickness, $\delta_{0}$, the evaporation and liquid flow discontinue in the non-evaporating region. We only consider one side of the meniscus because of the geometric symmetry and the following assumptions are introduced:

(i) quasi-steady two dimensional laminar flow with no slip at the wall,

(ii) the liquid and vapor flows are incompressible,

(iii) the vapor temperature at the interface, $T_{i v}$, which depends on the corresponding vapor pressure at the interface, $P_{i v}$, is non uniform along the interface and not equal to the bulk vapor temperature,

(iv) gravitational forces are neglected,

(v) the pure liquid completely wets the smooth solid surfaces which have a constant wall temperature, $T_{w}$, of $325 \mathrm{~K}$, and

(vi) the bulk vapor temperature, $T_{v}$, varies from 320 to $324 \mathrm{~K}$. The pressure, $P_{v}$, and the bulk vapor temperature are constant, for $x \leq 0$.

It is assumed that the flow is two-dimensional and by using a lubrication theory in the thin liquid film, it follows that the governing equation for momentum conservation is as follows

$$
\frac{\partial p_{l}}{\partial x}-\frac{\partial}{\partial y}\left(\mu_{l} \frac{\partial}{\partial y}\right)+S=\frac{\partial}{\partial y}(\rho u)
$$

where $u$ is the velocity and $S$ denotes the source term. The terms $\mu_{l}$ and $p_{l}$ are the liquid phase viscosity and pressure, respectively. The boundary conditions of Eq. (26) are: $\left.u_{l}\right|_{y=0}=0$, $u_{\delta}=\left.u_{v}\right|_{y=\delta}=\left.u_{l}\right|_{y=\delta}, \quad \tau_{i}=-\left.\mu_{v}\left(d u_{v} / d y\right)\right|_{y=\delta}=-\left.\mu_{l}\left(d u_{v} / d y\right)\right|_{y=\delta}$ and $\left.\left(d u_{v} / d y\right)\right|_{y=H / 2}=0$. It is also assumed that the wall temperature, $T_{w}$, is greater than the vapor temperature, $T_{v}$. For integration of Eq. (26), we integrate by substitution, so integrating of Eq. (26) with the above boundary conditions gives 


$$
\begin{aligned}
& u_{l}=\frac{\tau_{i}}{\mu_{l}} \int_{0}^{y} d w \int_{0}^{w} \frac{d p_{l}}{d x}(x, v) d v-\frac{1}{\mu_{l}} \int_{0}^{y} d w \int_{0}^{\delta} \frac{\partial p_{l}}{\partial x}(x, \xi) d \xi \\
& u_{v}=\frac{\tau_{i}}{\mu_{v}} \int_{0}^{y} d w \int_{0}^{w} \frac{d p_{v}}{d x}(x, v) d v-\frac{1}{\mu_{v}} \int_{0}^{y} d w \int_{0}^{H / 2} \frac{\partial p_{v}}{\partial x}(x, \xi) d \xi
\end{aligned}
$$

By changing integration order, Eqs. (27) \& (28) become

$$
\begin{gathered}
u_{l}=\frac{\tau_{i}}{\mu_{l}} \int_{0}^{y}(y-v) \frac{\partial p_{l}}{\partial x}(x, v) d v-y \frac{1}{\mu_{l}} \int_{0}^{\delta} \frac{\partial p_{l}}{\partial x}(x, \xi) d \xi \\
u_{v}=\frac{\tau_{i}}{\mu_{v}} \int_{0}^{y}(y-v) \frac{\partial p_{v}}{\partial x}(x, v) d v-y \frac{1}{\mu_{v}} \int_{0}^{H / 2} \frac{\partial p_{v}}{\partial x}(x, \xi) d \xi
\end{gathered}
$$

the mass flow rate at a given location can be expressed as

$$
\begin{gathered}
\dot{m}_{l}=\rho_{l} \int_{0}^{\delta} u d y=\rho_{l} \frac{\tau_{i}}{\mu_{l}} \int_{0}^{\delta} d y \int_{0}^{y}(y-v) \frac{\partial p_{l}}{\partial x}(x, v) d v-\rho_{l} \frac{1}{\mu_{l}} \int_{0}^{\delta} \frac{\partial p_{l}}{\partial x}(x, \xi) d \xi \int_{0}^{\delta} y d y \\
\dot{m}_{v}=\rho_{v} \int_{0}^{\delta} u d y=\rho_{v} \frac{\tau_{i}}{\mu_{v}} \int_{0}^{\delta} d y \int_{0}^{y}(y-v) \frac{\partial p_{v}}{\partial x}(x, v) d v-\rho_{v} \frac{1}{\mu_{v}} \int_{0}^{H / 2} \frac{\partial p_{v}}{\partial x}(x, \xi) d \xi \int_{0}^{H / 2} y d y
\end{gathered}
$$

The heat transfer rate by thin film evaporation occurring in the liquid-vapor interface $q^{\prime \prime}$

$$
\begin{gathered}
q^{\prime \prime}=\dot{m}_{l}^{\prime} h_{f g}=-\frac{\tau_{i}}{\mu_{l}} h_{f g} \int_{0}^{y}(y-v) \frac{\partial^{2} p_{l}}{\partial x^{2}}(x, v) d v+\frac{1}{\mu_{l}} y h_{f g} \int_{0}^{\delta} \frac{\partial p_{l}}{\partial x}(x, \xi) d \xi \\
q^{\prime \prime}=\dot{m}_{v}^{\prime} h_{f g}=-\frac{\tau_{i}}{\mu_{v}} h_{f g} \int_{0}^{y}(y-v) \frac{\partial^{2} p_{v}}{\partial x^{2}}(x, v) d v+\frac{1}{\mu_{v}} y h_{f g} \int_{0}^{H / 2} \frac{\partial p_{v}}{\partial x}(x, \xi) d \xi
\end{gathered}
$$

$q^{\prime \prime}$ in Eqs. (33) \& (34) is also equal to

$$
q^{\prime \prime}=k_{l} \frac{T_{w}-T_{i l}}{\delta}
$$

The Clausius-Clapeyron equation, i.e.

$$
\left(\frac{d p}{d T}\right)_{s a t}=\frac{h_{f g}}{\overline{T_{i}}\left(\frac{1}{\rho_{v}}-\frac{1}{\rho_{l}}\right)}
$$

where $h_{f g}$ is the latent heat of vaporization at the average phase change temperature $\bar{T}_{i}$. Since the liquid-vapor interface region is very thin, the average phase change temperature is approximated by the arithmetic average of the liquid and vapor temperatures at the interface i.e.,

$$
\bar{T}_{i}=\frac{\left(T_{i l}+T_{i v}\right)}{2}
$$


The liquid temperature at the interface derived from Eq. (36) and it is related to the interfacial pressure difference, $\Delta p_{i}$,

$$
(d T)_{i}=T_{i v} \frac{1}{h_{f g}}\left(\frac{1}{\rho_{v}}-\frac{1}{\rho_{l}}\right) \Delta p_{i}
$$

Integrating from the $T_{i v}$ to $T_{i l}$, results in

$$
T_{i l}-T_{i v}=T_{i v} \frac{\Delta p_{i}}{\rho_{v} h_{f g}} \quad\left(\rho_{l}>>\rho_{v}\right)
$$

Rearranging yields

$$
T_{i l}=T_{i v}\left(1+\frac{\Delta p_{i}}{\rho_{v} h_{f g}}\right)
$$

The pressure difference, $\Delta p_{i}=p_{i v}-p_{i l}$, between the vapor and liquid at the interface is expressed by the augmented Young-Laplace equation, i.e.,

where

$$
\Delta p_{i}=p_{i v}-p_{i l}=p_{c}-p_{d}
$$

$$
p_{c}=\sigma K=\frac{\sigma \frac{d^{2} \delta}{d x^{2}}}{\left[1+\left(\frac{d \delta}{d x}\right)^{2}\right]^{3 / 2}}
$$

and

$$
p_{d}=\frac{A}{\delta^{3}}
$$

respectively. Combining Eqs. (41)-(43) and differntiating with respect to $x$ gives,

$$
\delta^{\prime \prime \prime}=\frac{3 \delta^{\prime} \delta^{\prime 2}}{1+\delta^{\prime 2}}+\frac{\delta^{\prime \prime}}{\sigma} \frac{d \sigma}{d x}+\frac{1}{\sigma}\left(\frac{d P_{i l}}{d x}+\frac{d P_{i v}}{d x}-\frac{3 A}{\delta^{4}} \delta^{\prime}\right)\left(1+\delta^{\prime 2}\right)^{1.5}
$$

Substituting Eq. (40) into Eq. (35) end eliminating the $T_{s}, q^{\prime \prime}$,

Considering Eq. (41), Eq. (45) becomes

$$
q^{\prime \prime}=\frac{T_{w}-T_{i v}\left(1+\frac{\Delta p}{\rho_{v} h_{f g}}\right)}{\frac{\delta}{k_{l}}}
$$




$$
q^{\prime \prime}=\frac{T_{w}-T_{v}\left(1+\frac{p_{c}+p_{d}}{\rho_{v} h_{f g}}\right)}{\frac{\delta}{k_{l}}}
$$

Substituting Eq. (46) into Eqs. (33) \& (34)yields

$$
\begin{gathered}
\frac{T_{W}-T_{v}\left(1+\frac{p_{c}+p_{d}}{\rho_{v} h_{f g}}\right)}{\frac{\delta}{k_{l}}}=-\frac{\tau_{i}}{\mu_{l}} h_{f g} \int_{0}^{y}(y-v) \frac{\partial^{2} p_{l}}{\partial x^{2}}(x, v) d v+\frac{1}{\mu_{l}} y h_{f g} \int_{0}^{\delta} \frac{\partial p_{l}}{\partial x}(x, \xi) d \xi \\
\frac{T_{W}-T_{v}\left(1+\frac{p_{c}+p_{d}}{\rho_{v} h_{f g}}\right)}{\frac{\delta}{k_{l}}}=-\frac{\tau_{i}}{\mu_{v}} h_{f g} \int_{0}^{y}(y-v) \frac{\partial^{2} p_{v}}{\partial x^{2}}(x, v) d v+\frac{1}{\mu_{v}} y h_{f g} \int_{0}^{H / 2} \frac{\partial p_{v}}{\partial x}(x, \xi) d \xi
\end{gathered}
$$

Differentiating Eq. (41) with respect to $x$ yields

$$
\begin{aligned}
& \frac{\partial p_{i l}}{\partial x}=-\frac{\partial\left(p_{c}+p_{d}\right)}{\partial x} \\
& \frac{\partial p_{i v}}{\partial x}=\frac{\partial\left(p_{c}+p_{d}\right)}{\partial x}
\end{aligned}
$$

Substituting Eqs. (49) \& (50) into Eqs. (47) \& (48) give

$$
\begin{aligned}
& \rho_{l} \frac{\tau_{i}}{\mu_{l}} \int_{0}^{y}(y-v) \frac{\partial^{2}\left(p_{c}+p_{d}\right)}{\partial x^{2}}(x, v) d v-\rho_{l} \frac{1}{\mu_{l}} y \int_{0}^{\delta} \frac{\partial\left(p_{c}+p_{d}\right)}{\partial x}(x, \xi) d \xi=\frac{1}{h_{f g}}\left(T_{W}-T_{v}\left(1+\frac{p_{c}+p_{d}}{\rho_{v} h_{f g}}\right) /\left(\frac{\delta}{k_{l}}\right)\right) \\
& -\rho_{v} \frac{\tau_{i}}{\mu_{v}} \int_{0}^{y}(y-v) \frac{\partial^{2}\left(p_{c}+p_{d}\right)}{\partial x^{2}}(x, v) d v+\rho_{v} \frac{1}{\mu_{v}} y \int_{0}^{H / 2} \frac{\partial\left(p_{c}+p_{d}\right)}{\partial x}(x, \xi) d \xi=\frac{1}{h_{f g}}\left(T_{W}-T_{v}\left(1+\frac{p_{c}+p_{d}}{\rho_{v} h_{f g}}\right) /\left(\frac{\delta}{k_{l}}\right)\right)
\end{aligned}
$$

the disjoining pressure is the dominant parameter in evaporating thin-film region, which governs the fluid flow in the evaporating thin-film region. Also the absolute disjoining pressure is much larger than the capillary pressure, $p_{d}>>p_{c}$, especially when the curvature variation along the meniscus is very small, so Eqs.(51) \& (52) become

$$
\rho_{l} \frac{\tau_{i}}{\mu_{l}} \int_{0}^{y}(y-v) \frac{\partial^{2}\left(\frac{A}{\delta^{3}}\right)}{\partial x^{2}}(x, v) d v-\rho_{l} \frac{1}{\mu_{l}} y \int_{0}^{\delta} \frac{\partial\left(\frac{A}{\delta^{3}}\right)}{\partial x}(x, \xi) d \xi=\frac{1}{h_{f g}} T_{W}-\left(T_{v}\left(1+\frac{A}{\rho_{v} h_{f g} \delta^{3}}\right) /\left(\frac{\delta}{k_{l}}\right)\right)(53)
$$




$$
-\rho_{l} \frac{\tau_{i}}{\mu_{l}} \int_{0}^{v}(y-v) \frac{\partial^{2}\left(\frac{A}{\delta^{3}}\right)}{\partial x^{2}}(x, v) d v+\rho_{l} \frac{1}{\mu_{l}} y \int_{0}^{H / 2} \frac{\partial\left(\frac{A}{\delta^{3}}\right)}{\partial x}(x, \xi) d \xi=\frac{1}{h_{f g}} T_{W}-\left(T_{v}\left(1+\frac{A}{\rho_{v} h_{f g} \delta^{3}}\right) /\left(\frac{\delta}{k_{l}}\right)\right)
$$

Knowing that $v_{l}=\frac{\mu_{l}}{\rho_{l}}$, and integrating Eqs.(53) \& (54)

$$
\begin{aligned}
& \left(-\frac{4}{\delta^{2}}+\frac{3}{\delta}\right) \frac{d}{d x}\left(\frac{1}{\delta} \frac{\partial \delta}{\partial x}\right)=\frac{C_{1}-C_{2}}{\delta} \\
& \left(\frac{4}{\delta^{2}}-\frac{3}{\delta}\right) \frac{d}{d x}\left(\frac{1}{\delta} \frac{\partial \delta}{\partial x}\right)=\frac{C_{1}-C_{2}}{\delta}
\end{aligned}
$$

where $C_{1}$, and $C_{2}$ are

$$
\begin{gathered}
C_{1}=\frac{\frac{k_{l} \tau_{i} v_{l}}{A h_{f g}} \frac{\left(T_{W}-T_{v}\right)}{\delta}}{\frac{3}{\delta^{4}}}, \\
C_{2}=-\frac{\frac{k_{l} \tau_{i} v_{l}}{\rho_{l} h_{f g}^{2}} \frac{T_{v}}{\delta}}{\frac{3}{\delta^{4}}}
\end{gathered}
$$

respectively. Solving Eq. (55)\&(56), the slope of the thin film profile can be expressed as

$$
\frac{\partial \delta}{\partial x}=\sqrt{\frac{C_{1}}{4 \delta^{2}}-3 C_{2} \delta^{2}-\frac{3 C_{3}}{\delta}}
$$

Where $C_{3}$ is an integral constant, the constant $C_{3}$ can be expressed as

$$
C_{3}=\frac{C_{1}}{\delta_{0}}-\frac{C_{2}}{\delta_{0}^{4}}-\frac{d_{0}^{2}}{\delta_{0}^{2}}
$$

By knowing that $p_{d}=A / \delta_{0}^{3}=\left(h_{f s} / T_{v}\right)\left(T_{W}-T_{v}\right)$, The equilibrium thickness $\delta_{0}$ is as follows

$$
\delta_{0}=\left[\frac{A T_{i v}}{\rho_{v} h_{f g}\left(T_{W}-T_{i v}\right)}\right]^{1 / 3}=\left(\frac{C_{1}}{C_{2}}\right)^{1 / 3}
$$

Using Eqs. (46), (53), (57) and (58), and taking a derivative of Eq. (46), the analytical solution of $q^{\prime \prime}$, is as follows

$$
q^{\prime \prime}=\frac{A h_{f g}}{v_{l}}\left(\frac{60}{\delta^{6}}\right)+\frac{A h_{f g}}{v_{l}}\left(-\frac{12}{\delta^{5}}\right)=\frac{A h_{f g}}{v_{l}}\left(\frac{C_{1}}{3 \delta}-\frac{C_{2}}{4 \delta^{2}}\right)
$$

Thus, the optimum thickness $\delta_{q_{\max }}$,

$$
\delta=\delta_{q_{\max }^{m}}=3 / 20 \sqrt{\frac{C_{1}}{C_{2}}}=(3 / 20) \delta_{0}^{2 / 3}
$$


Considering Eq. (63), Eq. (62) can be rewritten as

$$
q^{\prime \prime}=\frac{A h_{f g}}{v_{l}}\left(\frac{C_{1}}{3 \delta}-\frac{C_{2}}{4 \delta^{2}}\right)=\frac{A h_{f g}}{v_{l}} \frac{C_{1}}{\delta}\left(\frac{1}{3}-\frac{C_{2}}{C_{1} \delta^{3}}\right)=\frac{A h_{f g}}{v_{l}} \frac{C_{1}}{\delta}\left(1-\frac{\delta_{0}^{3}}{\delta^{3}}\right)
$$

Substituting Eq. (57) into Eqs. (39) yields

$$
q^{\prime \prime}=\frac{A h_{f g}}{v_{l}} \frac{k_{l} v_{l}}{A h_{f g}} \frac{\left(T_{W}-T_{v}\right)}{\delta} \frac{\delta^{5}}{3}\left(1-\frac{\delta_{0}^{3}}{\delta^{3}}\right)=k_{l} \frac{\left(T_{W}-T_{v}\right)}{\delta} \frac{\delta^{5}}{3}\left(1-\frac{\delta_{0}^{3}}{\delta^{3}}\right)
$$

the dimensionless length, width and thickness are as follows

$$
x^{*}=\frac{x}{L}, y^{*}=H^{*}=\frac{y}{W}, \delta^{*}=\frac{\delta}{\delta_{0}}
$$

the dimensionless liquid pressure, vapor pressure, capillary pressure and disjoining pressure, are as follows

$$
P_{v}^{*}=1-\frac{P_{v}-P_{v, 0}}{P_{v, L}-P_{v, 0}}, P_{l}^{*}=1-\frac{P_{l}-P_{l, 0}}{P_{l, L}-P_{l, 0}}, P_{c}^{*}=\frac{P_{c}}{P_{c, L}}, P_{d}^{*}=\frac{P_{d}}{P_{d, 0}}
$$

and the dimensionless heat flux is

$$
q^{\prime \prime *}=\frac{q^{\prime \prime}}{q_{0}^{\prime \prime}}
$$

Utilizing the characteristic thickness $\delta_{0}$, and the characteristic heat flux, $q_{0}^{\prime \prime}=\frac{k_{l}\left(T_{W}-T_{v}\right)}{\delta_{0}}$, the dimensionless heat flux occurring in the evaporating thin film region can be expressed as

$$
q^{\prime \prime *}=\frac{1}{3 \delta^{*}}-\frac{1}{4 \delta^{* 2}}
$$

Considering $\frac{d q^{\prime \prime *}}{d \delta^{*}}=0$, the maximum dimensionless heat flux $q_{\max }^{\prime \prime *}$ can be determined as $-\frac{1}{3 \delta^{* 2}}+\frac{2}{\delta^{* 3}}=0$. When the thin film thickness is equal to $3 / 20$ times two third equilibrium thickness, $\delta_{0}^{(2 / 3)}$, the local heat flux through the evaporating thin film reaches its maximum. Letting $\delta^{*}=(3 / 20)^{2 / 3}$, the maximum dimensionless heat flux can be expressed as 
$q_{\max }^{\prime \prime *}=\left(\frac{3}{10}\right)^{2 / 3} \approx 0.44$. Considering Eqs. (41), (42), (43) \& (67), the following equation can be expressed as

$$
\left(\frac{d^{3} \delta^{*}}{d x^{* 3}}\right)=\frac{1}{\sigma}\left(\begin{array}{c}
\frac{d}{d x^{*}}\left(P_{v, 0}-\left(P_{v}^{*}-1\right)\left(P_{v, L}-P_{v, 0}\right)\right)-\frac{d}{d x^{*}}\left(P_{l, 0}-\left(P_{l}^{*}-1\right)\left(P_{l, L}-P_{l, 0}\right)\right) \\
+3 P_{d}^{*} P_{d, 0} \frac{A}{\delta^{* 3}} \frac{1}{\delta^{*}} \frac{d \delta^{*}}{d x^{*}}
\end{array}\right)-\frac{d^{3} \delta^{*}}{d x^{* 3}}\left[\left(\frac{d \delta^{*}}{d x^{*}}\right)^{2}\right]^{-3 / 2}
$$

Using Eqs. (60) \& (61), Eq. (59) yields

$$
\frac{\partial \delta}{\partial x}=\sqrt{\frac{C_{1} \delta_{0}^{2}}{4 \delta^{2}}-3 C_{2} \delta^{3} \delta_{0}^{-3}-\frac{3 \delta C_{3}}{\delta_{0}}}
$$

Nondimensionalizing Eq. (71) with the film thickness yields

$$
\frac{\partial \delta^{*}}{\partial x^{*}}=\sqrt{\frac{C_{1}}{4 \delta^{* 2}}-3 C_{2} \delta^{* 3}-3 C_{3} \delta^{*}}
$$

\subsection{RESULTS AND DISCUSSION}

Six prescribed boundary conditions for the film thickness, vapor and liquid pressures at $x=0$, and they are as follows

$$
\left.\delta\right|_{x=0}=\delta_{0},\left.(d \delta / d x)\right|_{x=0}=0,\left.K\right|_{x=0}=0, P_{v, 0}=P_{v, s a t}\left(T_{v, 0}\right), P_{l, 0}=P_{v, 0}-\frac{\bar{A}}{\delta_{0}^{3}}-\sigma K,\left.\delta^{*}\right|_{x^{*}=0}=1
$$

FC-72 was used as the working fluid. The calculations and predictions were based on the thermal properties, and dimensional parameters presented in Tables 3.1 \& 3.2. Eq. (23) and Eq. (49) were solved, with the boundary conditions in Eq. (52). To obtain the variation of thickness of thin film, we solved Eq. (23) with the boundary conditions of Eq. (52). The disjoining pressure can be neglected in the intrinsic meniscus region and the capillary pressure was dominant. Thus, we used the length $L$ when the value of $A / \delta^{3}$ was much greater than or equal to that of $\sigma K$ as the length of thin film region. Once $L$ was calculated, the film thickness at $x=L$, could be obtained. The basic variables $\left(\delta, \boldsymbol{u}_{l}, \boldsymbol{u}_{v}, P_{i l}, P_{i v}\right)$ were obtained by solving Eqs. (19), (20), 
(21), (22), and (34). $T_{i v}$ corresponds to the saturation vapor pressure at the interface. $T_{i l}$ was then obtained from Eqs. (30), (31), (32), and (33) with $T_{i v}$ and the new film profiles in Eq. (34). The calculated $\delta$ and $T_{i l}$ were then used to compute the liquid and vapor mass flow rates, $m_{l}$ and $m_{v}$.

Table 3.1. Properties of the working fluid FC-72

\begin{tabular}{|c|c|c|c|}
\hline Properties(unit) & Values & Properties (unit) & Values \\
\hline$A(J)$ & $1.0 \times 10^{-21}$ & $T_{v}(K)$ & 324.0 \\
\hline$k_{l}(\mathrm{~W} / \mathrm{mK})$ & 0.057 & $v_{l}\left(\mathrm{~m}^{2} / \mathrm{s}\right)$ & $0.2746 \times 10^{-6}$ \\
\hline$\rho_{v}\left(\mathrm{~kg} / \mathrm{m}^{3}\right)$ & 13.24 & $\rho_{l}\left(\mathrm{~kg} / \mathrm{m}^{3}\right)$ & 1680.0 \\
\hline$\sigma(\mathrm{N} / \mathrm{m})$ & $1.15 \times 10^{-2}$ & $h_{f g}(\mathrm{~J} / \mathrm{kg})$ & $9.437 \times 10^{4}$ \\
\hline
\end{tabular}

In order to verify the analytical solution derived herein, results predicted by Argade et al. [35] and Ma and Peterson [39] were used. Table 3.3 shows the comparison of analytical results of the heat flux, equilibrium film thickness, film thickness of evaporating film region and the dimensionless film thickness with results presented by Argade et al. [35]. The difference in the dimensionless film thickness between these two models is up to $25 \%$. The reason might be due to the dispersion constant. Ma and Peterson [39] have theoretically found the heat flux distribution along the evaporating thin-film region including the heat flux, equilibrium film thickness, and film thickness of evaporating film region. As shown in Table 3.3, the analytical predictions presented herein agree well with results of Ma and Peterson [39]. Figure 3.2 illustrates the superheat effect on the dimensionless equilibrium film thickness (nonevaporating film thickness). As shown, the equilibrium film thickness depends on the superheat. The dimensional equilibrium film thickness increases when superheat increases. 


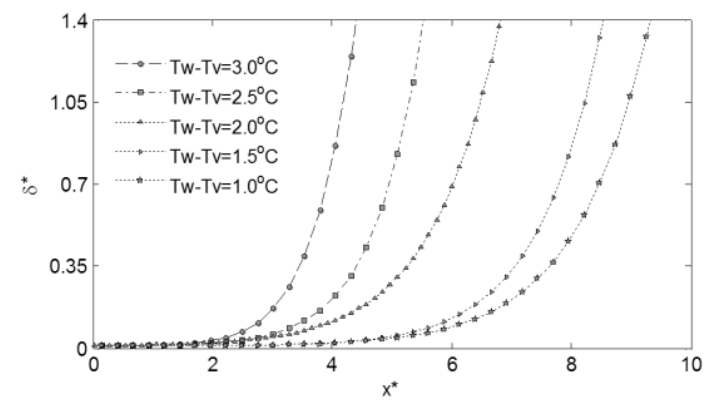

Fig 3.2.Dimensionless microlayer profile \& film thickness at various superheats

Table 3.2.Dimensional parameters \& calculated values

\begin{tabular}{|c|c|c|c|}
\hline $\begin{array}{c}\text { Parameters } \\
\text { (unit) }\end{array}$ & Values & Parameters (unit) & Values \\
\hline$L(m)$ & $9.52 \times 10^{-6}$ & $P_{v, 0}(P a)$ & $30.90 \times 10^{3}$ \\
\hline$\delta_{l}(m)$ & $3.21 \times 10^{-5}$ & $P_{v, L}(P a)$ & $30.80 \times 10^{3}$ \\
\hline$P_{l, 0}(P a)$ & $18.3 \times 10^{5}$ & $P_{d, 0}(P a)$ & $3.42 \times 10^{5}$ \\
\hline$P_{l, L}(P a)$ & $17.6 \times 10^{5}$ & $P_{c, L}(P a)$ & 261.32 \\
\hline
\end{tabular}

Figure 3.3 shows the superheat effect on the dimensionless heat flux through the evaporating thin region. From fig 3.3, it can be found that the maximum dimensionless heat flux is constant equal to about 0.44 , which will not depend on the superheat and is the same as the analytical solution. But the dimensionless location corresponding to the maximum dimensionless heat flux is different, which is a function of the superheat, and the dimensionless heat flux distribution profile shifts to the contact line.
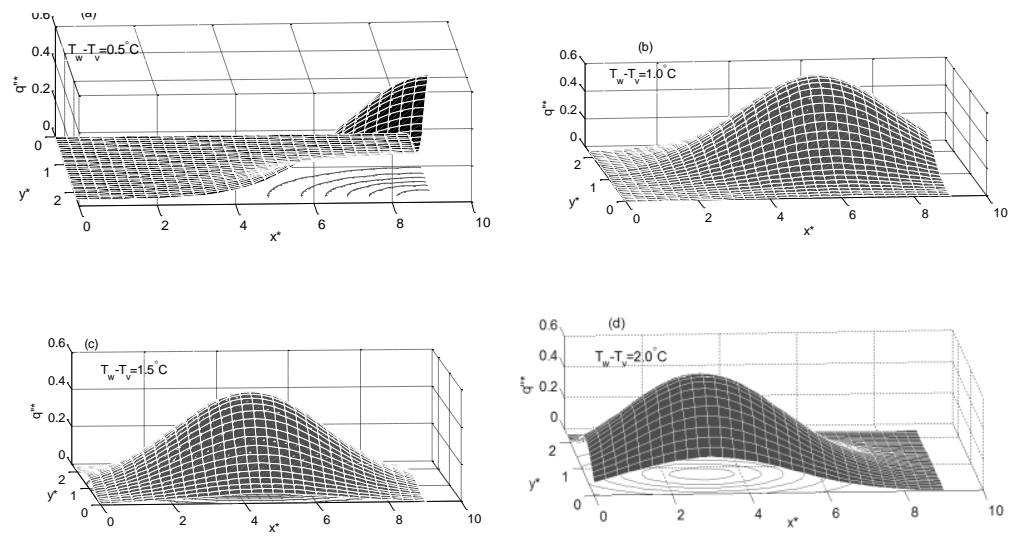

Fig 3.3. Dimensionless microlayer profile \& heat flux at various superheats 
In order to compensate for thin film evaporation, the relevant convective flow is the Marangoni recirculation or Marangoni vortex. Figure 3.4a-d shows the temperature profiles in the liquid of the evaporating thin film region. Marangoni flow is caused by temperature gradient induced surface tension gradient, which carries particles that are near the thin film liquid surface of the thin film toward the top of the thin film and then plunges them downward. The area with a higher surface tension draws more strongly on the surrounding working fluid than one with low surface tension. From the temperature profiles, it is shown that evaporation at the liquid-vapor interface leads to cooling of the liquid near the interface relative to the incoming liquid at the inlet. Liquid is fed steadily to the interface to sustain evaporation. The paths illustrate the fluid feeding as well as the Marangoni vortex set up near the interface due to thermocapillary convection. The liquid-vapor interface is at the vapor temperature except in the contact line of the thin-film region. The temperature of the liquid vapor interface is lowest in the central region, away from elliptical pins and solid walls. The Marangoni vortex is formed near the interface, with the vortex being non-uniform around the elliptic pin fins. Because of the surface tension difference, a shear stress at the liquid vapor interface due to the surface tension is produced, i.e., $\tau_{\sigma}=d \sigma / d x$.Due to this shear stress, liquid near one location will flow toward another location.
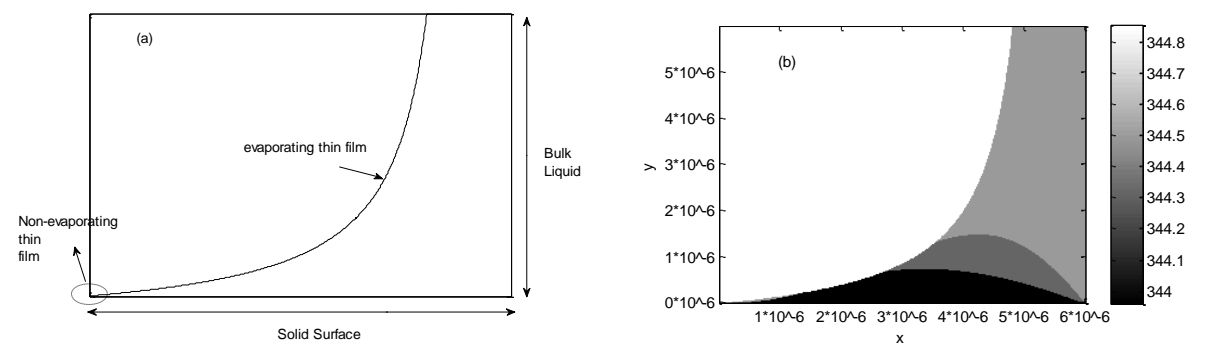


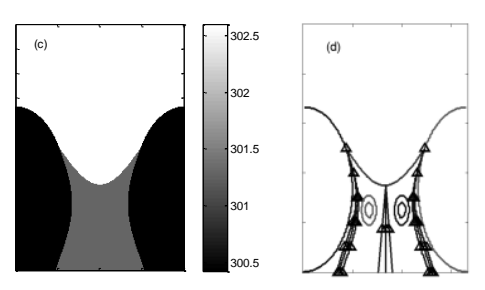

Fig 3.4. Temperature profiles and paths for evaporation

Figure 3.5(a-e) shows views of both $x$ and $y$ dimensionless coordinates and superheat for thin film profiles. At the initial superheat the nondimensional $x$ and $y$ thin film profile is uniform and shown in fig. 3.5a. When adding more superheat as shown in fig $3.5 \mathrm{~b}$, the thin film profile is not uniform and there are two crests in the approximate regions $\left(x^{*}<2.0, y^{*}<0.5\right)$ and $\left(x^{*}>8.0, y^{*}>1.5\right)$ and two shallow troughs in the approximate regions $\left(x^{*}>8.0, y^{*}<0.5\right)$ and $\left(x^{*}<2.0, y^{*}>1.5\right)$. Figures $3.5 \mathrm{c}$ and $3.5 \mathrm{~d}$ show that an increase in superheat causes the thin film profile to increase at 1 and 2 faster rates in the troughs relative to the crests, respectively. Examination of Eqs. (32) \& (33) show that change of views of both the dimensionless coordinates and superheat changes due to three distinct mechanisms: (i) due to surface tension gradient effects $\partial \sigma / \partial x$, (ii) due the disjoining pressure at $x=0$ which is determined by the superheat and fluid properties as shown in Eq. (51). (iii) due to the capillary pressure at $x=L$ where $\sigma K$ represents the capillary pressure limit, which is determined by the surface tension and the channel height. Figure $3.5 \mathrm{e}$ shows that that the thin film profile is optimum; it increases at a rate that is inversely proportional to fig. $3.5 \mathrm{~b}$. There are two crests in the approximate regions $\left(x^{*}>4.0, y^{*}<0.5\right)$ and $\left(x^{*}<2.0, y^{*}>1.5\right)$ and two shallow troughs in the approximate regions $\left(x^{*}<2.0, y^{*}<0.5\right)$ and $\left(x^{*}>4.0, y^{*}>1.5\right)$. 

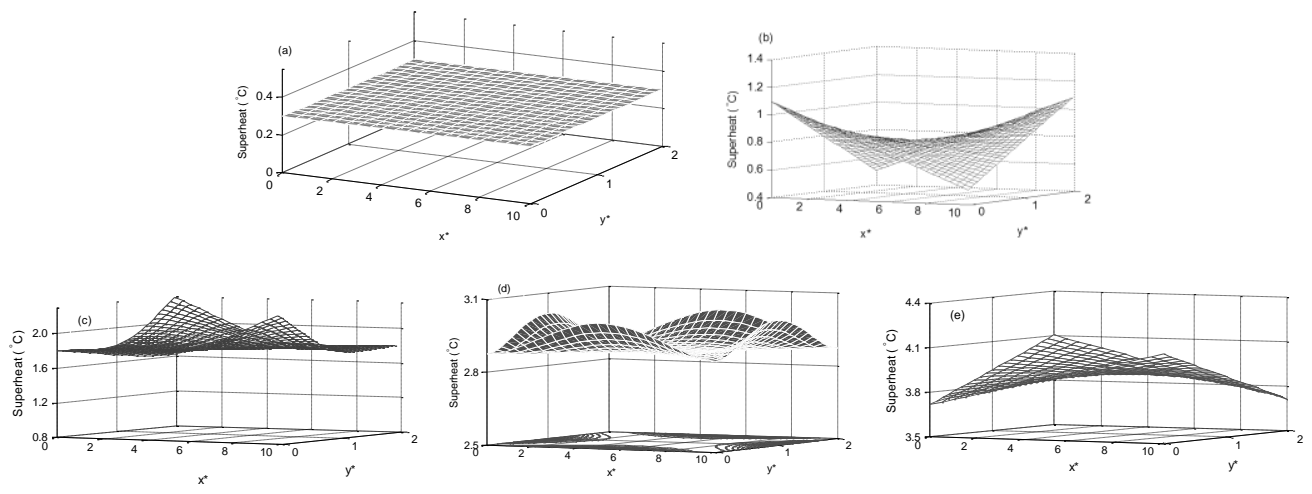

Fig 3.5. Superheat effects on thin film profiles

In order to explain the flow characteristics in thin film, the non-dimensional film thickness $\delta^{*}$, the non-dimensional vapor pressure $P_{v}^{*}$, and the non-dimensional liquid pressure $P_{l}^{*}$ with the non-dimensional channel length $x^{*}$ are shown in fig 3.6a, and the non-dimensional capillary and disjoining pressures $P_{c}^{*}, P_{d}^{*}$ with $x^{*}$ are shown in fig 3.6b. In fig 3.6, $x^{*}=0$ and $x^{*}=10$ denote the location of the beginning of thin film region (or the junction of the thin film region and the meniscus region) and the end of thin film region (or the beginning of the adsorbed region), respectively. It is can be seen in fig 3.6b that the film thickness $\delta^{*}$, which has initially a maximum value of $20.0 \mu \mathrm{m}$, decreases sharply until $x^{*}=4.0,(x=39.9 \mu \mathrm{m})$ and then it almost constant as the liquid flows. The film thickness is mainly influenced by the vapor and liquid phase pressures. Thus, the variation of film thickness can be explained by a pressure distribution in the thin film region. The liquid pressure $P_{l}^{*}$ is at its maximum and not changed for $x^{*}<4.0$, and then it decreases sharply until the end of thin film region. However, the vapor pressure $P_{v}^{*}$ gradually increases with $x^{*}$ to its maximum at $x^{*}=10.0$. The opposite pressure distribution, as shown in fig 3.6a, means that the liquid and vapor flow reversely because for the both sides of liquid and vapor, the friction is interacted at the liquid-vapor interface as the liquid 
in thin film flows. Figure $3.6 \mathrm{~b}$ shows that the non-dimensional capillary pressure $P_{c}^{*}$ decreases exponentially with $x^{*}$ and its distribution has the same trend as that of the film thickness. But the disjoining pressure $P_{d}^{*}$, which is not changed and has a minimum value for $x^{*}<4.0$, increases sharply until $x^{*}=8.0$ and then has a constant value until $x=0$. It is also found that the flow in thin film region is dominantly affected by the disjoining pressure because its value is higher than that of capillary pressure through all region of thin film. As it can be seen in fig. 3.6b, the capillary pressure plays a role in the flow characteristic in thin film region, although its value is much smaller than that of disjoining pressure. Therefore, when the flow and heat transfer characteristics in the thin film region are analyzed, the capillary pressure effect should be considered for more accurate results.
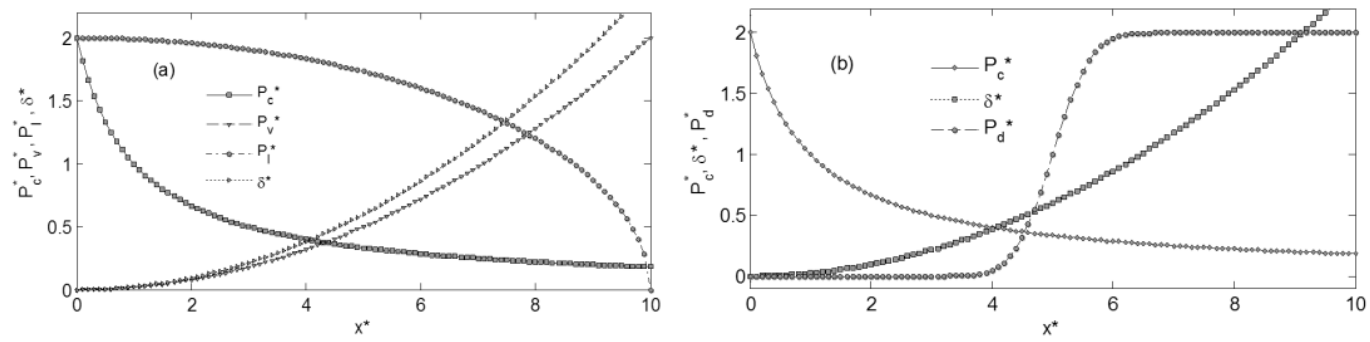

Fig 3.6. Dimensionless film thickness, liquid, vapor, capillary and disjoining pressures

Figure 3.7 shows that the maximum liquid pressure difference, which correspond to the maximum heat transfer in the evaporating thin film, and it is determined by the disjoining pressure at $x=0$ and the capillary pressure at $x=L . A / \delta_{0}^{3}$ represents the disjoining pressure limit which is determined by the superheat and fluid properties as shown in Eq. (51). $\sigma K_{\infty}$ represents the capillary pressure limit, which is determined by the surface tension and the channel height. The maximum liquid pressure difference continuously increases with the superheat. The maximum liquid pressure difference peaks at about $5^{\circ} \mathrm{C}$ superheat. 


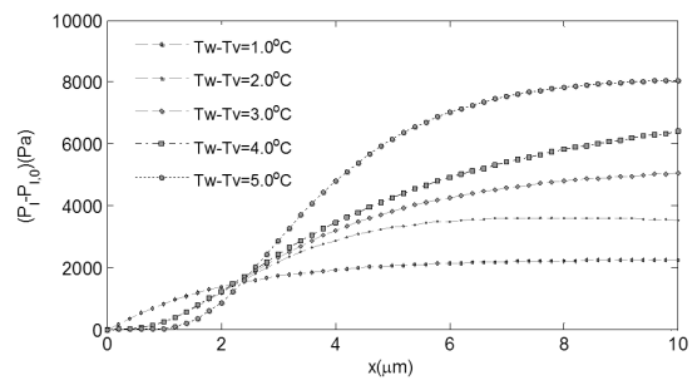

Fig 3.7. Liquid pressure difference at various superheats

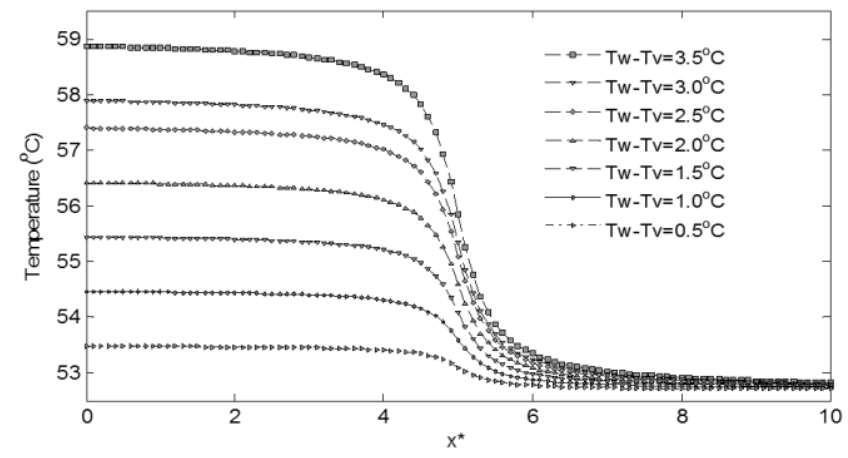

Fig 3.8. Interface temperature of the thin film at various superheats

The interface temperature of the thin film is illustrated in fig 3.8. As shown, the temperature remains constant for the equilibrium film region and then decreases drastically until it approaches the interface temperature of the bulk fluid region. This drastic decrease in temperature occurs when the film profile increases in thickness. The curvature profiles at various superheats are shown in fig 3.9. The film curvature is zero in the non-evaporating region, then increases and goes to a maximum in the evaporating thin film region. Figure 3.9 shows there exists a maximum curvature for a given superheat. There exists a higher curvature because at a higher superheat, the mass flow rate through the thin film region increase, resulting in pressure loss due to viscous forces to increase. The pumping pressure due to the disjoining pressure effect compensated for the viscous pressure loss and the pressure variations due to the curvature effect as the curvature aids in pulling liquid to the bulk fluid region. This causes larger changes in the film profile to produce large disjoining pressure gradients i.e. $K(x)_{\max }<\left(A / \delta_{0}^{3}\right)$. It is shown that 
the maximum curvature continuously increases with increasing the superheat. The curvature curve reaches a peak near $5^{\circ} \mathrm{C}$ superheat and decreases as superheat decreases.

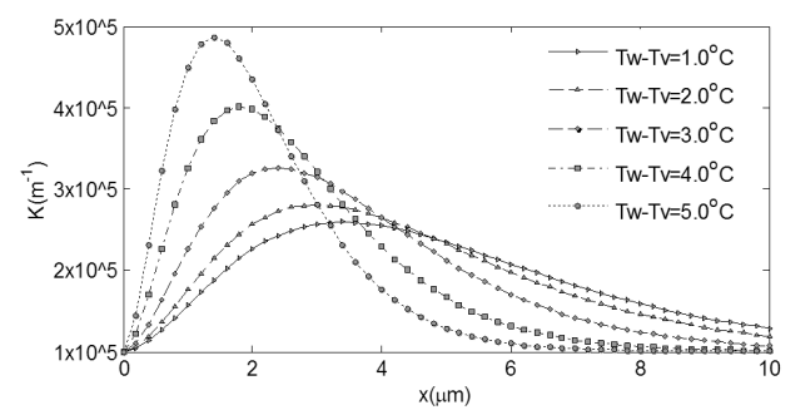

Fig 3.9. Curvature profiles at various superheats

Table 3.3.Comparison of the calculated results with previous models

\begin{tabular}{|c|c|c|c|}
\hline $\begin{array}{c}\text { Parameters } \\
\text { (unit) }\end{array}$ & $\begin{array}{c}\text { Ma \& Peterson } \\
{[39]}\end{array}$ & Argade et al [35] & Current Model \\
\hline$\delta_{0}(m)$ & $9.0 \times 10^{-7}$ & $2.2 \times 10^{-8}$ & $10.0 \times 10^{-7}$ \\
\hline$q^{\prime \prime}\left(\mathrm{W} / \mathrm{m}^{2}\right)$ & $1.6 \times 10^{7}$ & $N / A$ & $16 \times 10^{7}$ \\
\hline$\delta^{*}$ & 1.50 & 1.05 & 1.40 \\
\hline$\delta_{\max }(m)$ & $2.0 \times 10^{-8}$ & $2.2 \times 10^{-8}$ & $3.0 \times 10^{-8}$ \\
\hline
\end{tabular}

\subsection{SUMMARY}

Non dimensional analytical investigations were performed for heat flux and pressure distributions. The maximum liquid pressure difference continuously increases with the superheat. The maximum liquid pressure difference peaks at about $5^{\circ} \mathrm{C}$ superheat. The curvature and interface temperature profiles were different at different superheats. A decrease in temperature occurred when the thin film profile increased in thickness. Both the $x$ and $y$ dimensionless coordinates and superheat at various thin film profiles were viewed and there were two crests in regions $\left(x^{*}>4.0, y^{*}<0.5\right)$ and $\left(x^{*}<2.0, y^{*}>1.5\right)$, and two shallow troughs in regions $\left(x^{*}<2.0, y^{*}<0.5\right)$ and $\left(x^{*}>4.0, y^{*}>1.5\right)$ where thin film profile was optimum. 


\section{CHAPTER FOUR: NON-DIMENSIONAL ANALYSIS OF TWO-PHASE FLOW AND CONDENSATION HEAT TRANSFER IN POROUS MEDIUM}

\subsection{INTRODUCTION}

Vapor flows into the porous medium and condenses into liquid. During this process, there exists vapor phase, liquid-vapor phase, liquid phase and a condensation zone as shown in Fig. 4.1.The vapor condensation is assumed to occur at the condensation forepart (of a negligibly small thickness) $x_{F}$ located between the liquid and two-phase regions. In the liquid region, the liquid saturation is assumed as unity $s=1$. In the two-phase region, the liquid saturation varies, both phases are mobile, and the capillary pressure is nonzero $p_{c} \neq 0$. No liquid motion is allowed in the vapor region. In this chapter, the movement of the condensation forepart $x_{F}$ into the porous medium is investigated.

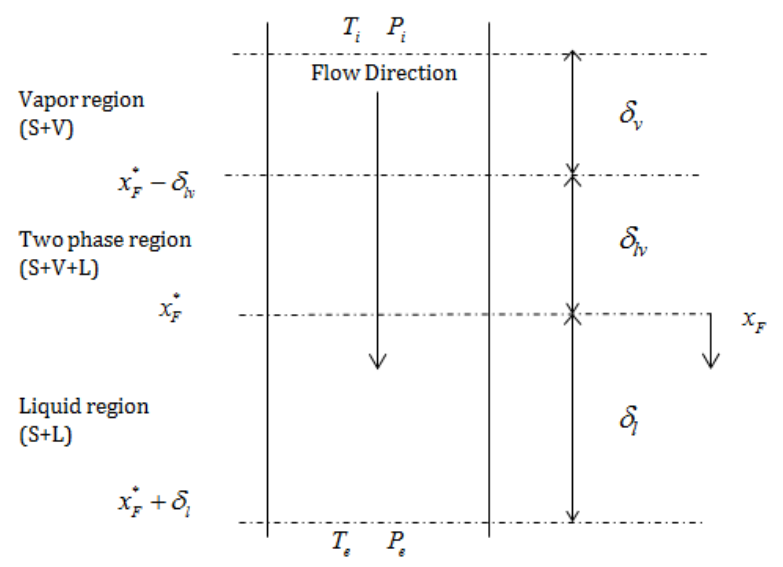

Fig 4.1. Fluid Flow Regions

\subsection{ANALYSIS}

\subsubsection{THEORETICAL MODEL}

By introducing the macroscopic and microscopic inertial terms, the following equation for liquid phase flow in porous media is introduced 


$$
\frac{\rho_{l}}{\varepsilon s}\left(\frac{\partial\left\langle u_{l}\right\rangle}{\partial t}+\left\langle u_{l}\right\rangle \cdot \nabla\left\langle u_{l}\right\rangle\right)=-\nabla\langle p\rangle^{l}+\rho_{l} g-\frac{\mu_{l}}{K_{l}}\left\langle u_{l}\right\rangle-\frac{\rho_{l}}{K_{l i}}\left|\left\langle u_{l}\right\rangle\right|\left\langle u_{l}\right\rangle
$$

where $\frac{\rho_{l}}{\varepsilon s}\left(\frac{\partial\left\langle u_{l}\right\rangle}{\partial t}+\left\langle u_{l}\right\rangle \cdot \nabla\left\langle u_{l}\right\rangle\right)$ is liquid phase macroscopic inertial force, $-\nabla\langle p\rangle^{l}$ is liquid phase pore pressure gradient, $\rho_{l} g$ is liquid phase body force, $\frac{\rho_{l}}{K_{l i}}\left|\left\langle u_{l}\right\rangle\right|\left\langle u_{l}\right\rangle$ is liquid phase microscopic inertial force. The liquid phase microscopic interfacial shear stress $\frac{\mu_{l}}{K_{l}}\left\langle u_{l}\right\rangle$ has a permeability term that relates flow rate and fluid physical properties (i.e. viscosity) to pressure gradient. The permeability term is part of the proportionality constant in Darcy's law and can be expressed as,

$$
K_{l}=\mu_{l}\left\langle u_{l}\right\rangle / \nabla\langle p\rangle^{l}
$$

By rearranging Eq. (75), pressure gradient for liquid phase in Darcy's law is as follows

$$
\nabla\langle p\rangle^{l}=\frac{\mu_{l}}{K_{l}}\left\langle u_{l}\right\rangle
$$

which is the liquid phase microscopic interfacial shear stress presented in Eq. (74).

Assuming stationary state $(\partial / \partial t=0)$ and 1-D flow (and small velocity gradient $(\partial / \partial x=0)$, Eq. (74) becomes

$$
0=-\frac{\partial p_{l}}{\partial x}+\rho_{l} g-\frac{\mu_{l}}{K_{l}} u_{l}-\frac{\rho_{l}}{K_{l i}} u_{l}^{2}
$$

Rearranging Eq. (77) yields,

Knowing that

$$
\frac{\partial p_{l}}{\partial x}=\rho_{l} g-\frac{\mu_{l}}{K_{l}} u_{l}-\frac{\rho_{l}}{K_{l i}}\left|u_{l}\right| u_{l}
$$

$$
K_{l}=K K_{r l}
$$

and 


$$
\frac{\rho_{l}}{K_{l i}}\left|u_{l}\right| u_{l}=\rho_{l} \frac{C_{E}}{K^{1 / 2} K_{r l i}}\left|u_{l}\right| u_{l}
$$

Eq. (78) can be expressed as,

$$
\frac{\partial p_{l}}{\partial x}=\rho_{l} g-\frac{\mu_{l}}{K K_{r l}} u_{l}-\frac{\rho_{l} C_{E}}{K^{1 / 2} K_{r l i}}\left|u_{l}\right| u_{l}
$$

Introducing dimensionless pressure

$$
p_{l}^{*}=\frac{p_{l}-p_{e}}{\sigma}\left(\frac{K}{\varepsilon}\right)^{1 / 2}
$$

and dimensionless position for liquid phase

$$
x^{*}=x \frac{B o}{(K \varepsilon)^{1 / 2}}
$$

Substituting Eq. (82) and Eq. (83) into Eq. (81) yields,

$$
\left(\frac{\sigma B o}{K}\right) \frac{\partial p_{l}^{*}}{\partial x^{*}}=\rho_{l} g-\frac{\mu_{l}}{K K_{r l}} u_{l}-\frac{\rho_{l} C_{E}}{K^{1 / 2} K_{r l i}}\left|u_{l}\right| u_{l}
$$

Substituting Eq. (84) yields,

$$
\frac{\partial p_{l}^{*}}{\partial x^{*}}=\frac{\rho_{l} g K}{\sigma B o}-\frac{\mu_{l} u_{l} K}{\sigma B o} \frac{1}{K K_{r l}}-\left(\frac{K}{\sigma B o}\right) \frac{\rho_{l} C_{E}}{K^{1 / 2} K_{r l i}}\left|u_{l}\right| u_{l}
$$

Substituting Eq. (124) and Eq. (126) into Eq. (85)

$$
\frac{\partial p_{l}^{*}}{\partial x^{*}}=\frac{B o_{l}}{B o}-\frac{C a_{l}}{B o} \frac{1}{K_{r l}}-\frac{C a_{l}}{B o} C_{E} \frac{\rho_{l} u_{l} K^{1 / 2}}{\mu_{l}} \frac{1}{K_{r l i}}
$$

and rearranging yields,

$$
\frac{\partial p_{l}^{*}}{\partial x^{*}}=\frac{B o_{l}}{B o}-\frac{C a_{l}}{B o}\left(\frac{1}{K_{r l}}+\frac{C_{E} \mathrm{Re}_{l}}{K_{r l i}}\right)
$$

By introducing the macroscopic and microscopic inertial terms, the following equation for liquid phase flow in porous media is introduced 


$$
\frac{\rho_{v}}{\varepsilon(1-s)}\left(\frac{\partial}{\partial t}\left\langle u_{v}\right\rangle+\left\langle u_{v}\right\rangle \cdot \nabla\left\langle u_{v}\right\rangle\right)=-\nabla\langle p\rangle^{v}+\rho_{v} g-\frac{\mu_{v}}{K_{v}}\left\langle u_{v}\right\rangle-\frac{\rho_{v}}{K_{v i}}\left|\left\langle u_{v}\right\rangle\right|\left\langle u_{v}\right\rangle
$$

where $\frac{\rho_{v}}{\varepsilon s}\left(\frac{\partial\left\langle u_{v}\right\rangle}{\partial t}+\left\langle u_{v}\right\rangle \cdot \nabla\left\langle u_{v}\right\rangle\right)$ is vapor phase macroscopic inertial force, $-\nabla\langle p\rangle^{v}$ is vapor phase pore pressure gradient, $\rho_{v} g$ is vapor phase body force, $\frac{\rho_{v}}{K_{v i}}\left|\left\langle u_{v}\right\rangle\right|\left\langle u_{v}\right\rangle$ is vapor phase microscopic inertial force. The vapor phase microscopic interfacial shear stress $\frac{\mu_{v}}{K_{v}}\left\langle u_{v}\right\rangle$ has a permeability term that relates flow rate and fluid physical properties (i.e. viscosity) to pressure gradient. The permeability term is part of the proportionality constant in Darcy's law and can expressed as,

$$
K_{v}=\mu_{v}\left\langle u_{v}\right\rangle / \nabla\langle p\rangle^{v}
$$

By rearranging Eq. (89), pressure gradient for vapor phase in Darcy's law is as follows

$$
\nabla\langle p\rangle^{v}=\frac{\mu_{v}}{K_{v}}\left\langle u_{v}\right\rangle
$$

which is the vapor phase microscopic interfacial shear stress presented in Eq. (88).

Assuming stationary state $(\partial / \partial t=0)$ and 1-D flow (and small velocity gradient $(\partial / \partial x=0)$, Eq. (88) becomes

$$
0=-\frac{\partial p_{v}}{\partial x}+\rho_{v} g-\frac{\mu_{v}}{K_{v}} u_{v}-\frac{\rho_{v}}{K_{v i}} u_{v}^{2}
$$

Rearranging Eq. (91) yields,

$$
\frac{\partial p_{v}}{\partial x}=\rho_{v} g-\frac{\mu_{v}}{K_{v}} u_{v}-\frac{\rho_{v}}{K_{v i}}\left|u_{v}\right| u_{v}
$$


For the entire system, the two phases and the interfacial layer, the conservation of thermal energy is expressed as

$$
\begin{gathered}
{\left[(1-\varepsilon)\left(\rho c_{p}\right)_{s}+\varepsilon s\left(\rho c_{p}\right)_{l}+\varepsilon(1-s) \sum_{1=1}^{n_{r}}\left\langle\rho_{i}\right\rangle^{v}\left(c_{p}\right)_{i v}\right] \frac{\partial\langle T\rangle}{\partial t}+\left[\left(\rho c_{p}\right)_{l}\left\langle u_{l}\right\rangle+\sum_{1=1}^{n_{r}}\left\langle\rho_{i v}\right\rangle^{v}\left(c_{p}\right)_{i v}\left\langle u_{v}\right\rangle\right] \cdot \nabla\langle T\rangle+\Delta i_{\mathrm{lv}}\langle\dot{n}\rangle} \\
=\nabla \cdot\left[K_{e}+\left(\rho c_{p}\right)_{l} D^{d}\right] \cdot \nabla\langle T\rangle+\langle\dot{s}\rangle
\end{gathered}
$$

Dividing each term in Eq. (93) by $\left(\rho c_{p}\right)_{s}$ and replacing $\nabla$ by $\partial / \partial x$, Eq. (93) can be rewritten as

$$
\begin{gathered}
{\left[(1-\varepsilon)+\varepsilon s \frac{\left(\rho c_{p}\right)_{l}}{\left(\rho c_{p}\right)_{s}}+\varepsilon(1-s) \frac{\left(\rho c_{p}\right)_{v}}{\left(\rho c_{p}\right)_{s}}\right] \frac{\partial T}{\partial t}+\left[\frac{\left(\rho c_{p}\right)_{l}}{\left(\rho c_{p}\right)_{s}} u_{l}+\frac{\left(\rho c_{p}\right)_{v}}{\left(\rho c_{p}\right)_{s}} u_{l}\right] \cdot \frac{\partial T}{\partial x}+\frac{\Delta i_{\mathrm{lv}}\langle\dot{n}\rangle}{\left(\rho c_{p}\right)_{s}}=\frac{1}{\left(\rho c_{p}\right)_{s}}(94)} \\
\frac{\partial}{\partial x}\left\{\left[K_{e}+\left(\rho c_{p}\right)_{l} D^{d}\right] \frac{\partial T}{\partial x}\right\}+\frac{1}{\left(\rho c_{p}\right)_{s}}\langle\dot{s}\rangle
\end{gathered}
$$

using,

$$
\begin{aligned}
& x^{*}=x \frac{B o}{(K \varepsilon)^{1 / 2}} \\
& t^{*}=t \frac{B o^{2} \sigma}{(K \varepsilon)^{1 / 2} \mu_{l}}
\end{aligned}
$$

and

$$
T^{*}=\frac{T-T_{l}}{T_{F, s a t}-T_{l}}=\frac{T-T_{l}}{\Delta T}
$$

differentiating $T$ with respect to $t$, Eq. (97) yields

$$
\frac{\partial T}{\partial t}=\Delta T \frac{\partial T^{*}}{\partial t}
$$

and by rewriting right term of Eq. (98) and differentiating Eq. (96), the following is obtained

$$
\Delta T \frac{\partial T^{*}}{\partial t^{*}} \frac{d t^{*}}{d t}=\Delta T \frac{B o^{2} \sigma}{(K \varepsilon)^{1 / 2} \mu_{l}} \frac{\partial T^{*}}{\partial t^{*}}
$$

Thus, 


$$
\frac{\partial T}{\partial t}=\Delta T \frac{B o^{2} \sigma}{(K \varepsilon)^{1 / 2} \mu_{l}} \frac{\partial T^{*}}{\partial t^{*}}
$$

differentiating $T$ with respect to $x$, Eq. (97) yields

$$
\frac{\partial T}{\partial x}=\Delta T \frac{\partial T^{*}}{\partial x}
$$

and by rewriting right term of Eq. (101) and differentiating Eq. (95), the following is obtained

$$
\Delta T \frac{\partial T^{*}}{\partial x^{*}} \frac{d x^{*}}{d x}=\Delta T \frac{B o}{(K \varepsilon)^{1 / 2}} \frac{\partial T^{*}}{\partial x^{*}}
$$

Thus,

$$
\frac{\partial T}{\partial x}=\Delta T \frac{B o}{(K \varepsilon)^{1 / 2}} \frac{\partial T^{*}}{\partial x^{*}}
$$

Substituting Eq. (100) and Eq. (103) into Eq. (94) yields

$$
\begin{gathered}
\Delta T \frac{B o^{2} \sigma}{(K \varepsilon)^{1 / 2} \mu_{l}}\left[(1-\varepsilon)+\varepsilon s\left(\rho c_{p}\right)_{l}^{*}+\varepsilon(1-s)\left(\rho c_{p}\right)_{l}^{*}\right] \frac{\partial T^{*}}{\partial t^{*}}+\Delta T \frac{B o}{(K \varepsilon)^{1 / 2}}\left[\left(\rho c_{p}\right)_{l}^{*} u_{l}+\left(\rho c_{p}\right)_{v}^{*} u_{l}\right] \cdot \frac{\partial T^{*}}{\partial x^{*}} \\
+\frac{\Delta i_{\mathrm{lv}}\langle\dot{n}\rangle}{\left(\rho c_{p}\right)_{s}}=\frac{1}{\left(\rho c_{p}\right)_{s}} \Delta T \frac{B o}{(K \varepsilon)^{1 / 2}}\left[K_{e} \frac{\partial T^{*}}{\partial x^{*}}+\Delta T \frac{B o}{(K \varepsilon)^{1 / 2}}\left(\rho c_{p}\right)_{l} D^{d} \frac{\partial^{2} T^{*}}{\partial x^{* 2}}\right]+\frac{1}{\left(\rho c_{p}\right)_{s}}\langle\dot{s}\rangle
\end{gathered}
$$

first right of Eq. (104) has a novel heat flux term $\left(\partial^{2} T^{*} / \partial x^{* 2}\right)$ which is obtained by differentiating Eq. (103) and combining with Eq. (94) which make my model very unique. By dividing each term in Eq. (104) by $\Delta T \frac{B o \sigma}{(K \varepsilon)^{1 / 2} \mu_{l}}$ and rearranging,

$$
\begin{gathered}
{\left[(1-\varepsilon)+\varepsilon s\left(\rho c_{p}\right)_{l}^{*}+\varepsilon(1-s)\left(\rho c_{p}\right)_{l}^{*}\right] B o \frac{\partial T^{*}}{\partial t^{*}}+\frac{\mu_{l}}{\sigma}\left[\left(\rho c_{p}\right)_{l}^{*} u_{l}+\left(\rho c_{p}\right)_{v}^{*} u_{l}\right] \cdot \frac{\partial T^{*}}{\partial x^{*}}+\frac{(K \varepsilon)^{1 / 2} \mu_{l} \Delta i_{\mathrm{lv}}\langle\dot{n}\rangle}{B o\left(\rho c_{p}\right)_{s} \sigma \Delta T}} \\
=\frac{1}{\left(\rho c_{p}\right)_{s}} \frac{\mu_{l}}{\sigma}\left[K_{e} \frac{\partial T^{*}}{\partial x^{*}}+\frac{B o}{(K \varepsilon)^{1 / 2}}\left(\rho c_{p}\right)_{l} D^{d} \frac{\partial^{2} T^{*}}{\partial x^{* 2}}\right]+\frac{(K \varepsilon)^{1 / 2} \mu_{l}}{B o\left(\rho c_{p}\right)_{s} \sigma \Delta T}\langle\dot{s}\rangle
\end{gathered}
$$


and by substituting, $C a_{l}=\mu_{l} u_{l} / \sigma, C a_{v}=\mu_{v} u_{v} / \sigma$, and rearranging, Eq.(105) can be expressed as,

$$
\begin{gathered}
{\left[(1-\varepsilon)+\varepsilon s\left(\rho c_{p}\right)_{l}^{*}+\varepsilon(1-s)\left(\rho c_{p}\right)_{l}^{*}\right] B o \frac{\partial T^{*}}{\partial t^{*}}+\left[C a_{l}\left(\rho c_{p}\right)_{l}^{*}+C a_{g}\left(\rho c_{p}\right)_{v}^{*}\right] \cdot \frac{\partial T^{*}}{\partial x^{*}}+\frac{(K \varepsilon)^{1 / 2} \mu_{l} \Delta i_{\mathrm{lv}}\langle\dot{n}\rangle}{B o\left(\rho c_{p}\right)_{s} \sigma \Delta T}} \\
=\frac{1}{\left(\rho c_{p}\right)_{s}}\left[\left(\rho c_{p}\right)_{l}\right]\left(\rho c_{p}\right)_{l} \frac{B o}{\left(\frac{(K \varepsilon)^{1 / 2} \sigma}{\mu_{l}}\right)} \frac{\partial^{2} T^{*}}{\partial x^{* 2}}+\frac{(K \varepsilon)^{1 / 2} \mu_{l}}{B o\left(\rho c_{p}\right)_{s} \sigma \Delta T}\langle\dot{s}\rangle
\end{gathered}
$$

Knowing that

and

$$
K_{v}=K K_{r v}
$$

$$
\frac{\rho_{v}}{K_{l i}}\left|u_{v}\right| u_{v}=\rho_{v} \frac{C_{E}}{K^{1 / 2} K_{r i}}\left|u_{v}\right| u_{v}
$$

Eq. (92) can be expressed as,

$$
\frac{\partial p_{v}}{\partial x}=\rho_{v} g-\frac{\mu_{v}}{K K_{r v}} u_{v}-\frac{\rho_{v} C_{E}}{K^{1 / 2} K_{r v i}}\left|u_{v}\right| u_{v}
$$

Introducing dimensionless pressure

$$
p_{v}^{*}=\frac{p_{v}-p_{e}}{\sigma}\left(\frac{K}{\varepsilon}\right)^{1 / 2}
$$

and dimensionless position for vapor phase

$$
x^{*}=x \frac{B o}{(K \varepsilon)^{1 / 2}}
$$

Substituting Eq. (110) and Eq. (111) into Eq. (109),

$$
\left(\frac{\sigma B o}{K}\right) \frac{\partial p_{v}^{*}}{\partial x^{*}}=\rho_{v} g-\frac{\mu_{v}}{K K_{r v}} u_{v}-\frac{\rho_{l} C_{E}}{K^{1 / 2} K_{r v i}}\left|u_{v}\right| u_{v}
$$

and rearranging yields,

$$
\frac{\partial p_{v}^{*}}{\partial x^{*}}=\frac{\rho_{v} g K}{\sigma B o}-\frac{\mu_{v} u_{v} K}{\sigma B o} \frac{1}{K K_{r v}}-\left(\frac{K}{\sigma B o}\right) \frac{\rho_{v} C_{E}}{K^{1 / 2} K_{r v i}}\left|u_{v}\right| u_{v}
$$


Substituting Eq. (124) and Eq. (126) into Eq. (113)

$$
\frac{\partial p_{v}^{*}}{\partial x^{*}}=\frac{B o_{v}}{B o}-\frac{C a_{v}}{B o} \frac{1}{K_{r v}}-\frac{C a_{v}}{B o} C_{E} \frac{\rho_{v} u_{v} K^{1 / 2}}{\mu_{v}} \frac{1}{K_{r v i}}
$$

and rearranging yields,

$$
\frac{\partial p_{v}^{*}}{\partial x^{*}}=\frac{B o_{v}}{B o}-\frac{C a_{v}}{B o}\left(\frac{1}{K_{r v}}+\frac{C_{E} \operatorname{Re}_{v}}{K_{r v i}}\right)
$$

The dimensionless, two-phase flow conservation equations for the liquid- and gas-phase for mass are

$$
\begin{gathered}
\varepsilon B o \frac{\partial s}{\partial t^{*}}+\frac{\partial C a_{l}}{\partial x^{*}}=\dot{n}_{l}^{*} \\
-\varepsilon B o \frac{\partial s}{\partial t^{*}}+\mu^{*} \frac{\partial C a_{v}}{\partial x^{*}}=\dot{n}_{v}^{*}
\end{gathered}
$$

for momentum derived from Eq. (74) to Eq. (115) are

$$
\begin{gathered}
\frac{\partial p_{l}^{*}}{\partial x^{*}}=\frac{B o_{l}}{B o}-\frac{C a_{l}}{B o}\left(\frac{1}{K_{r l}}+\frac{C_{E} \mathrm{Re}_{l}}{K_{r i}}\right) \\
\frac{\partial p_{v}^{*}}{\partial x^{*}}=\frac{B o_{v}}{B o}-\frac{C a_{v}}{B o}\left(\frac{1}{K_{r v}}+\frac{C_{E} \mathrm{Re}_{v}}{K_{r v i}}\right)
\end{gathered}
$$

and for the thermal energy (under the assumption of local thermal equilibrium) derived from Eq. (93) to Eq. (106) are

$$
\begin{gathered}
{\left[(1-\varepsilon)+\varepsilon s\left(\rho c_{p}\right)_{l}^{*}+\varepsilon(1-s)\left(\rho c_{p}\right)_{v}^{*}\right] B o \frac{\partial T^{*}}{\partial t^{*}}+\left[s C a_{l}\left(\rho c_{p}\right)_{l}^{*}+(1-s) C a_{v}\left(\rho c_{p}\right)_{v}^{*} \mu^{*}\right] \frac{\partial T^{*}}{\partial x^{*}}} \\
=\left[s+(1-s)\left(\rho c_{p}\right)_{v l}^{*} \frac{D_{v}}{D_{l}}\right]\left(\rho c_{p}\right)_{l}^{*} \frac{B o}{\left(P e_{l}\right)_{m}} \frac{\partial^{2} T^{*}}{\partial x^{* 2}}+\frac{\dot{n}_{l}}{J a}
\end{gathered}
$$

Rewriting dimensionless position and time,

$$
x^{*}=x \frac{B o}{(K \varepsilon)^{1 / 2}}, t^{*}=t \frac{B o^{2} \sigma}{(K \varepsilon)^{1 / 2} \mu_{l}}
$$

dimensionless temperature, 


$$
T^{*}=\frac{T-T_{l}}{T_{F, s a t}-T_{l}}=\frac{T-T_{l}}{\Delta T}
$$

dimensionless pressure,

$$
p_{l}^{*}=\frac{p_{l}-p_{c}}{\sigma}\left(\frac{K}{\varepsilon}\right)^{1 / 2}, p_{v}^{*}=\frac{p_{v}-p_{c}}{\sigma}\left(\frac{K}{\varepsilon}\right)^{1 / 2}
$$

Capillary numbers:

$$
C a_{l}=\frac{\mu_{l} u_{l}}{\sigma}, C a_{v}=\frac{\mu_{v} u_{v}}{\sigma}
$$

Reynolds numbers:

$$
R e_{l}=\frac{\rho_{l} u_{l} K^{1 / 2}}{\mu_{l}}, R e_{v}=\frac{\rho_{v} u_{v} K^{1 / 2}}{\mu_{v}}
$$

Bond numbers,

$$
B o=\frac{\left(\rho_{l}-\rho_{v}\right) g K}{\sigma}, B o_{l}=\frac{\rho_{l} g K}{\sigma}, B o_{v}=\frac{\rho_{v} g K}{\sigma}, \rho^{*}=\frac{\rho_{v}}{\rho_{l}}
$$

dimensionless heat capacity,

$$
\left(\rho c_{p}\right)_{l}^{*}=\frac{\left(\rho c_{p}\right)_{l}}{\left(\rho c_{p}\right)_{s}},\left(\rho c_{p}\right)_{v}^{*}=\frac{\left(\rho c_{p}\right)_{v}}{\left(\rho c_{p}\right)_{s}},\left(\rho c_{p}\right)_{v l}^{*}=\frac{\left(\rho c_{p}\right)_{v}}{\left(\rho c_{p}\right)_{l}}
$$

and dimensionless parameters,

$$
\mu^{*}=\frac{\mu_{l}}{\mu_{v}},\left(P e_{l}\right)_{m}=\frac{(K \varepsilon)^{1 / 2} \sigma}{D_{l} \mu_{l}}, J a=\frac{c_{p l} \Delta T}{\Delta i_{\mathrm{lv}}} \frac{1}{\left(\rho c_{p}\right)_{l}^{*}}
$$

The modified Peclet number $\left(P e_{l}\right)_{m}$ is based on the total axial diffusivity and the square root of the absolute permeability and $\Delta T$ is the difference between the initial temperature and the saturation temperature evaluated at forepart pressure. 
Vapor region, $\left(0 \leq x^{*} \leq x_{F}^{*}-\delta_{l v}^{*}\right)$

In this region the liquid remains in the pores after the passing of the fore part and is considered immobile; no phase change occurs and the capillary pressure is assumed constant.

Then

$$
\begin{gathered}
\dot{n}_{l}=\dot{n}_{v}=0, u_{l}=0\left(C a_{l}=0\right), u_{v}=u_{\mathrm{v}, 0}\left(C a_{v}=C a_{\mathrm{v}, 0}\right), \\
p_{c}^{*}=p_{v}^{*}-p_{l}^{*}=\text { const }, T^{*}=T_{\text {sat }}^{*}\left(p_{v}\right)
\end{gathered}
$$

Since $p_{c}^{*}=p_{v}^{*}-p_{l}^{*}$ is constant, subtracting Eq. (118) from Eq. (119) will produce

$$
\frac{\partial\left(p_{v}^{*}-p_{l}^{*}\right)}{\partial x^{*}}=\frac{\left(B o_{v}-B o_{l}\right)}{B o}-\frac{C a_{v, 0}}{B o}\left(\frac{1}{K_{r v}}+\frac{C_{E} \mathrm{Re}_{\mathrm{v}, 0}}{K_{r v i}}\right)=0
$$

Or

$$
\frac{\partial p_{c}^{*}}{\partial x^{*}}-1-\frac{C a_{v, 0}}{B o}\left(\frac{1}{K_{r v}}+\frac{C_{E} \operatorname{Re}_{v, 0}}{K_{r v i}}\right)=0, \quad B o=B o_{l}-B o_{v}
$$

Eq. (131) is used to determine the continuous but immobile liquid saturation $s_{i m}=s_{i m}\left(x^{*}\right)$, and, as evident, this distribution depends on the ratio of the Bond and capillary numbers.

Two-phase region, $\left(x_{F}^{*}-\delta_{l v}^{*} \leq x^{*} \leq x_{F}^{*}\right)$

In this region, both the liquid and vapor are mobile, and no phase change occurs:

$$
\dot{n}_{l}=\dot{n}_{v}=0, T^{*}=1
$$

The velocity of the condensation forepart can be regarded as the pore velocity of the liquid at the forepart.

Using a moving coordinate system based on the condensation-fore part speed, i.e.

$$
x_{1}=x-u_{F} t
$$


Where

$$
u_{F}=\frac{u^{*} \sigma}{u_{l}}
$$

Substituting Eq. (121) into Eq. (133) yields

$$
\frac{(K \varepsilon)^{1 / 2}}{B o} x_{1}^{*}=\frac{(K \varepsilon)^{1 / 2}}{B o} x^{*}-\frac{\sigma}{\mu_{l}} u_{F}^{*} \frac{(K \varepsilon)^{1 / 2} \mu_{l}}{B o^{2} \sigma} t^{*}
$$

Rearranging yields

$$
x_{1}^{*}=x^{*}-\left(u_{F}^{*} / B o\right) t^{*}
$$

Eq. (116) is rewritten as

$$
\varepsilon B o \frac{\partial s}{\partial x_{1}^{*}} \frac{\partial x_{1}^{*}}{\partial t^{*}}+\frac{\partial C a_{l}}{\partial x_{1}^{*}} \frac{\partial x_{1}^{*}}{\partial x^{*}}=0
$$

by differentiating

$$
\frac{\partial x_{1}^{*}}{\partial t^{*}}=-u_{F}^{*} / B o, \quad \frac{\partial x_{1}^{*}}{\partial x^{*}}=1
$$

And by substituting Eq. (98) into Eq.(97), Eq. (74) can be written as

$$
\frac{\partial}{\partial x_{1}^{*}}\left(C a_{l}-\varepsilon s u_{F}^{*}\right)=0
$$

Now, Eq. (117) is rewritten as

$$
-\varepsilon B o \frac{\partial s}{\partial x_{1}^{*}} \frac{\partial x_{1}^{*}}{\partial t^{*}}+\mu^{*} \frac{\partial C a_{l}}{\partial x_{1}^{*}} \frac{\partial x_{1}^{*}}{\partial x^{*}}=0
$$


Substituting Eq. (138) into Eq. (140), Eq. (117) can be written as

$$
\frac{\partial}{\partial x_{1}^{*}}\left(\mu^{*} C a_{v}+\varepsilon s u_{F}^{*}\right)=0
$$

Solution of Eq. (139) is as follows, from Eq. (139)

$$
\left(C a_{l}-\varepsilon s u_{F}^{*}\right)=\text { const } .
$$

or

$$
C a_{l}=s u_{F}^{*}, u_{F}^{*}=\varepsilon u_{F},(s=1, \text { at the front })
$$

Then,

$$
\text { const. }=0
$$

Hence,

$$
C a_{l}=\varepsilon s u_{F}^{*}
$$

From Eq. (141),

$$
\left(\mu^{*} C a_{g}+\varepsilon s u_{F}^{*}\right)=\text { const } .
$$

or

$$
\mu^{*} C a_{v}=\mu^{*} C a_{v, 0}-\varepsilon u_{F}^{*},\left(u_{v}=u_{v, 0}-\varepsilon u_{F}\right),(s=1, \text { at the front })
$$

Then,

$$
\text { const. }=\left(\mu^{*} C a_{v, 0}-\varepsilon u_{F}^{*}\right)+\varepsilon u_{F}^{*}=\mu^{*} C a_{v, 0}
$$

Hence,

$$
\mu^{*} C a_{v}=\mu^{*} C a_{v, 0}-\varepsilon s u_{F}^{*}
$$

The liquid saturation distribution is determined from the following equation, which is derived by subtracting Eq. (118) from Eq. (119): 


$$
\frac{\partial\left(p_{v}^{*}-p_{l}^{*}\right)}{\partial x^{*}}=\frac{\left(B o_{v}-B o_{l}\right)}{B o}-\frac{C a_{v}}{B o}\left(\frac{1}{K_{r v}}+\frac{C_{E} \mathrm{Re}_{v}}{K_{r v i}}\right)+\frac{C a_{l}}{B o}\left(\frac{1}{K_{r l}}+\frac{C_{E} \mathrm{Re}_{l}}{K_{r l i}}\right)
$$

Rearranging yields

$$
\frac{\partial p_{c}^{*}}{\partial x^{*}}=-\frac{C a_{v}}{B o}\left[\left(\frac{1}{K_{r v}}+\frac{C_{E} \operatorname{Re}_{v}}{K_{r v i}}\right)-\frac{C a_{l}}{C a_{v}}\left(\frac{1}{K_{r l}}+\frac{C_{E} \mathrm{Re}_{l}}{K_{r l i}}\right)+\frac{B o}{C a_{v}}\right]
$$

Or

$$
\frac{\partial p_{c}^{*}}{\partial x^{*}}=\frac{\partial p_{c}^{*}}{\partial x_{1}^{*}} \frac{\partial x_{1}^{*}}{\partial x^{*}}=\frac{\partial p_{c}^{*}}{\partial x_{1}^{*}}
$$

Condensation forepart, $\left(x^{*}=x_{F}^{*}\right)$

At the forepart, the energy released due to phase change is transferred to the condensed liquid, i.e.

$$
-\left.\frac{B o}{\left(P e_{l}\right)_{m}} J a\left(\rho c_{p}\right)_{l}^{*} \frac{\partial T^{*}}{\partial x_{1}^{*}}\right|_{x_{1}^{*}=0^{+}}=\rho^{*}\left(\mu^{*} C a_{v, 0}-\varepsilon u_{F}^{*}\right)
$$

Through the integration of Eq. (162) given below, this heat flux is also given through

$$
(1-\varepsilon) u_{F}^{*}-\varepsilon\left(\rho c_{p}\right)_{l}^{*} \Delta C a_{l}-\left.\frac{B o}{\left(P e_{l}\right)_{m}} J a\left(\rho c_{p}\right)_{l}^{*} \frac{\partial T^{*}}{\partial x_{1}^{*}}\right|_{x_{1}^{*}=0^{+}}
$$

The second term on the left-hand side of Eq. (154) represents the sensible heat of the condensed liquid, $C a_{v, 0}\left(\right.$ i.e. $\left.u_{v, 0}\right)$ is determined from Eqs. (153) and (154), which give

$$
\rho^{*}\left(\mu^{*} C a_{v, 0}-\varepsilon u_{F}^{*}\right)=J a\left[(1-\varepsilon) u_{F}^{*}-\varepsilon\left(\rho c_{p}\right)_{l}^{*} \Delta C a_{l}\right]
$$

Liquid region, $\left(x_{F}^{*} \leq x^{*} \leq x_{F}^{*}+\delta_{l}^{*}\right)$ 
In the liquid region the porous medium is completely saturated with the subcooled liquid, and then

$$
s=1, \dot{n}_{l}=\dot{n}_{v}=0, \mu_{l}=\mu_{l, i}
$$

The thickness of the liquid region $\delta_{l}^{*}$, which begins at $x_{F}^{*}$, is determined from the total mass balance and is

$$
\delta_{l}^{*}=\operatorname{Jax}_{F}^{*} \frac{1-\varepsilon}{\varepsilon}-\int_{0}^{x_{F}^{*}} s d x^{*}
$$

The pore liquid velocity is the sum of the condensation forepart velocity and the rate of increase in the thickness of the liquid region. Then, the superficial velocity $u_{l}\left(\right.$ i.e. $\left.C a_{l}\right)$ and $\Delta C a_{l}$ are determined from

$$
C a_{l}=\varepsilon\left(u_{F}^{*}+\Delta C a_{l}\right)=\varepsilon\left(u_{F}^{*}+\frac{\partial \delta_{l}^{*}}{\partial t^{*}}\right)=\varepsilon u_{F}^{*}\left(1+J a \frac{1-\varepsilon}{\varepsilon}-\frac{1}{u_{F}^{*}} \frac{\partial}{\partial t^{*}} \int_{0}^{x_{F}^{*}} s d x^{*}\right)
$$

The energy equation is transformed to the moving coordinate system as

$$
\frac{\partial T^{*}}{\partial t^{*}}=\frac{\partial T^{*}}{\partial x_{1}^{*}} \frac{\partial x_{1}^{*}}{\partial t^{*}}=-\frac{u_{F}^{*}}{B o} \frac{\partial T^{*}}{\partial x_{1}^{*}}
$$

Taking into account that $s=1$

$$
\frac{\partial T^{*}}{\partial x^{*}}=\frac{\partial T^{*}}{\partial x_{1}^{*}} \frac{\partial x_{1}^{*}}{\partial x^{*}}=\frac{\partial T^{*}}{\partial x_{1}^{*}}, \quad \text { where }, \frac{\partial x_{1}^{*}}{\partial t^{*}}=-u_{F}^{*} / \text { Bo, } \frac{\partial x_{1}^{*}}{\partial x^{*}}=1
$$

The energy conservation, Eq. (120) then follows that,

$$
\left[(1-\varepsilon)+\varepsilon\left(\rho c_{p}\right)_{l}^{*}\right] B o\left(-\frac{u_{F}^{*}}{B o}\right) \frac{\partial T^{*}}{\partial x_{1}^{*}}+C a_{l}\left(\rho c_{p}\right)_{l}^{*} \frac{\partial T^{*}}{\partial x_{1}^{*}}=\left(\rho c_{p}\right)_{l}^{*} \frac{B o}{\left(P e_{l}\right)_{m}} \frac{\partial^{2} T^{*}}{\partial x_{1}^{* 2}}
$$

Rearranging yields, 


$$
\left\{-\left[(1-\varepsilon)+\varepsilon\left(\rho c_{p}\right)_{l}^{*}\right] u_{F}^{*}+C a_{l}\left(\rho c_{p}\right)_{l}^{*}\right\} \frac{\partial T^{*}}{\partial x_{1}^{*}}=\left(\rho c_{p}\right)_{l}^{*} \frac{B o}{\left(P e_{l}\right)_{m}} \frac{\partial^{2} T^{*}}{\partial x_{1}^{* 2}}
$$

In the transformation to the moving coordinate system, the variation of $u_{F}^{*}$ with time can be included as,

$$
\frac{\partial x_{1}^{*}}{\partial x^{*}}=1-\frac{\partial u_{F}^{*}}{\partial x^{*}} \frac{t^{*}}{B o} \frac{\partial x_{1}^{*}}{\partial t^{*}}=-\frac{u_{F}^{*}}{B o} \frac{\partial u_{F}^{*}}{\partial t^{*}} \frac{t^{*}}{B o}
$$

Integrating this for $0 \leq x^{*} \leq \infty$ and using the zero gradient conditions in the far-field gives Eq. (154). Eq. (162) is subject to the boundary conditions $T^{*}=1$ at $x_{1}^{*}=0$ and $T^{*} \rightarrow 0$ as $x_{1}^{*} \rightarrow \infty$ and by assuming,

$$
a=\left(\rho c_{p}\right)_{l}^{*} \frac{B o}{\left(P e_{l}\right)_{m}}, b=-\left[(1-\varepsilon)+\varepsilon\left(\rho c_{p}\right)_{l}^{*}\right] u_{F}^{*}+C a_{l}\left(\rho c_{p}\right)_{l}^{*}
$$

The solution to Eq. (153) becomes

$$
a \frac{\partial^{2} T^{*}}{\partial x_{1}^{* 2}}-b \frac{\partial T^{*}}{\partial x_{1}^{*}}=0
$$

Assuming

$$
T^{*} \propto e^{s x_{1}^{*}}
$$

Substituting Eq. (166) into Eq. (165) and differentiating,

$$
a\left(s^{2} e^{s x_{1}^{*}}+e^{s x_{1}^{*}}(0)\right)-b\left(s e^{s x_{1}^{*}}\right)=0
$$

Dividing by $e^{s x_{1}^{*}}$ and rearranging yields,

$$
a s^{2}-b s=0
$$

Or

$$
\left(s-\frac{b}{a}\right)-(s-0)=0
$$


So $s_{1}=\frac{b}{a}$ and $s_{2}=0$, the general solution to Eq. (165),

$$
T^{*}\left(x_{1}^{*}\right)=C_{1} e^{(b / a) x_{1}^{*}}+C_{2}
$$

Boundary conditions give $C_{1}=1, C_{2}=0$, hence,

$$
T^{*}\left(x_{1}^{*}\right)=e^{(b / a) x_{1}^{*}}
$$

Substituting Eq. (164) into Eq. (171) yields,

$$
T^{*}\left(x_{1}^{*}\right)=\exp \left\{\frac{-\left[(1-\varepsilon)+\varepsilon\left(\rho c_{p}\right)_{l}^{*}\right] u_{F}^{*}+C a_{l}\left(\rho c_{p}\right)_{l}^{*}}{\left(\rho c_{p}\right)_{l}^{*} \frac{B o}{\left(P e_{l}\right)_{m}}} x_{1}^{*}\right\}
$$

And substituting Eq. (158) into Eq. (172) results in,

$$
T^{*}\left(x_{1}^{*}\right)=\exp \left\{\frac{-\left[(1-\varepsilon)+\varepsilon\left(\rho c_{p}\right)_{l}^{*}\right] u_{F}^{*}+\left(\rho c_{p}\right)_{l}^{*} \varepsilon u_{F}^{*}\left(1+J a \frac{1-\varepsilon}{\varepsilon}-\frac{1}{u_{F}^{*}} \frac{\partial}{\partial t^{*}} \int_{0}^{x_{F}^{*}} s d x^{*}\right)}{\left(\rho c_{p}\right)_{l}^{*} \frac{B o}{\left(P e_{l}\right)_{m}}} x_{1}^{*}\right\}
$$

Rearranging yields,

$$
T^{*}\left(x_{1}^{*}\right)=\exp \left\{-\left(P e_{l}\right)_{m} \frac{(1-\varepsilon)+\varepsilon\left(\rho c_{p}\right)_{l}^{*}-\varepsilon\left(\rho c_{p}\right)_{l}^{*}-\varepsilon\left(\rho c_{p}\right)_{l}^{*}\left(J a \frac{1-\varepsilon}{\varepsilon}-\frac{1}{u_{F}^{*}} \frac{\partial}{\partial t^{*}} \int_{0}^{x_{F}^{*}} s d x^{*}\right)}{\left(\rho c_{p}\right)_{l}^{*}} \frac{u_{F}^{*} x_{1}^{*}}{B o}\right\}
$$




\subsubsection{SOLUTION AND PREDICTION PROCEDURE}

Since the forepart speed $u_{F}$ is not known, $u_{F}$ is first obtained by iteration. At a forepart location $0 \leq x_{F}^{*} \leq x_{e}^{*}$, first a guessed value $u_{F}^{*}$ is used in equation (134) and then the thermal diffusivity in the vapor region is obtained from equation (128) with $C a_{l}\left(\right.$ i.e. $\left.u_{l}\right)$ and $C a_{v}\left(\right.$ i.e. $\left.u_{v}\right)$ from equations (145) and (149). Further, the liquid and vapor-phase velocities, $u_{l}\left(\right.$ i.e. $\left.C a_{l}\right) C a_{v}\left(\right.$ i.e. $\left.u_{v}\right)$, are found in terms of the condensation-forepart speed $u_{F}^{*}$ through equations (145), (149), (155) and (158). Then, the value of $u_{F}^{*}$ is reiterated and updated. By solving equations (118) and (119) using the boundary conditions $p^{*}=p_{0}^{*}$ at the inlet and $p^{*}=0$ at the exit and by using dimensionless front $\operatorname{speed} u_{F}^{*}$, dimensionless temperature distribution is obtained from equation (174).

\subsection{RESULTS\& DISCUSSION}

In the two phase region, there is a jump of vapor pressure gradient $-\partial p_{v}^{*} / \partial x^{*}$ and it is larger than the vapor pressure gradient in the vapor region. Vapor pressure gradient $-\partial p_{v}^{*} / \partial x^{*}$ drops rapidly in the liquid phase region. However, because the thickness of the two-phase region is small $\delta_{l v}^{*}<x_{F}^{*}$, pressure drop is much smaller, and the front speed $u_{F}^{*}$ is mainly determined by the pressure drop within the vapor region. With this pressure drop increasing, the initial vapor speed $C a_{v, 0}\left(\right.$ i.e. $\left.u_{v, 0}\right)$ and front location speed $u_{F}^{*}$ decreases as dimensionless length $x^{*}$ and front location $x_{F}^{*}$ increase as shown in Fig $4.2 \&$ Fig 4.3. The thickness of the two-phase region 
increases with increase in front location $x_{F}^{*}$, because the capillary force in equation (151) becomes dominant as front speed $u_{F}^{*}$ decreases.

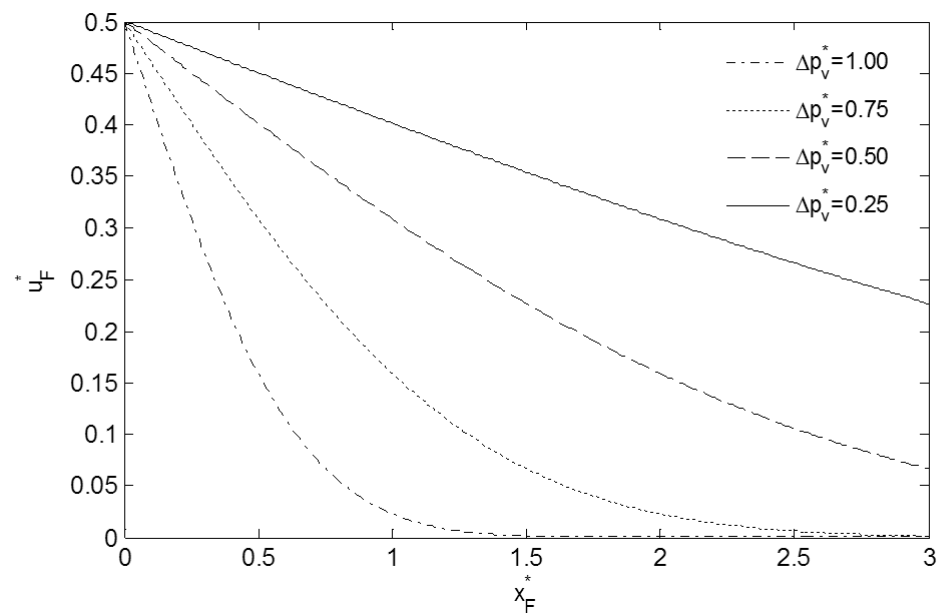

Fig 4.2. Variations of $u_{F}^{*}$ with dimensionless front location

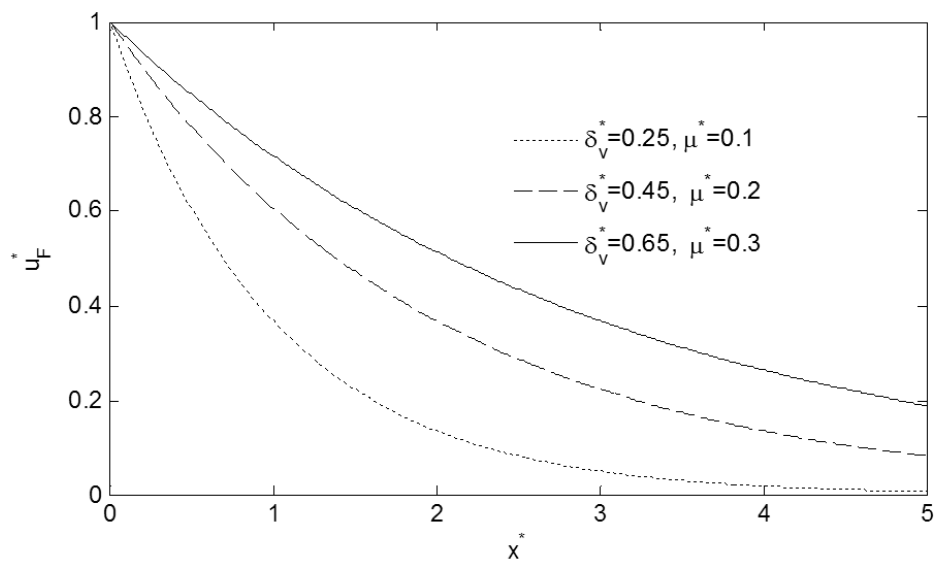

Fig 4.3. Variations of $u_{F}^{*}$ with dimensionless location

For the flow of vapor, $u_{V}^{*} / B o$ is a function of $x_{F}^{*} J a / p_{0}^{*}$ and there is a change of the ratio of $u_{F}^{*} / B o$ with respect to $x_{F}^{*} J a / p_{0}^{*}$ in equations (157), (163) and (174). The front speed $u_{F}^{*}$ decreases with $x_{F}^{*}$. Further, the thickness of the liquid region increase with $x_{F}^{*}$ and $u_{l}^{*}$ as shown in Fig. 4.4 \& Fig. 4.5 (the pressure drops in these regions have only a secondary effect on $u_{F}^{*}$ ). 
Consequently, $u_{F}^{*} / B o$ decreases with $x_{F}^{*}$. Since the enthalpy of the flow is much larger than the heat loss to the porous medium, the effect of the flow heat loss on $u_{F}^{*}$ is negligibly small. The vapor pressure drops rapidly within a short distance in the liquid region as shown in Fig. 4.6 and Fig. 4.7. In the transformation to the moving coordinate system, the variation of $u_{F}^{*}$ with time is included as shown in equations (133) and (135). Before liquid saturates, there is a jump at the beginning of the two-phase region. There is variation for the thicknesses of the liquid and twophase regions, $\delta_{l}^{*}$ and $\delta_{l v}^{*}$, for vapor and when $x \neq 0, p^{*}\left(x^{*}, t^{*} \rightarrow \infty\right)=p_{0}^{*}$. The dimensionless thickness of the liquid phase $\delta_{l}^{*}$ increases with the front location $x_{F}^{*}$. The liquid saturation in the liquid-phase region is for $u_{F}^{*} / B o$ (equal to 0.177 ), and corresponds to $B o / C a_{v, 0}=1.18$ in equation (151). $B o / C a_{v, 0}$ is independent of $J a$, and increases with $B o$, because of the increase in the inertial term. After the front passes through, the liquid begins to increase with increase in $x_{F}^{*}$. This saturation of liquid is determined from the balance between the gravity force and the sum of the viscous $\left(\mu^{*}\right)$ and inertial $\left(u^{*}\right)$ forces in equation (131).

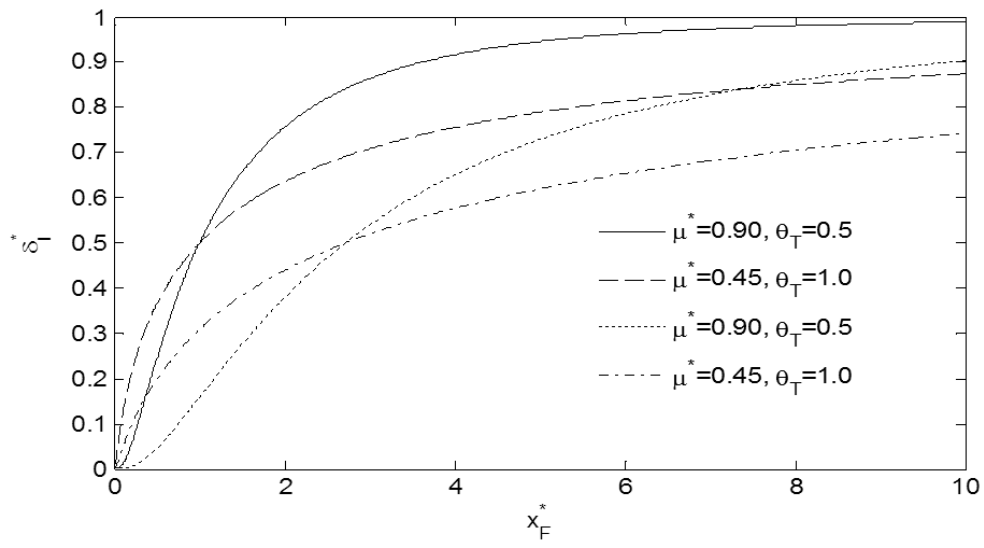

Fig 4.4. Variations of $\delta_{l}^{*}$ with dimensionless front location 


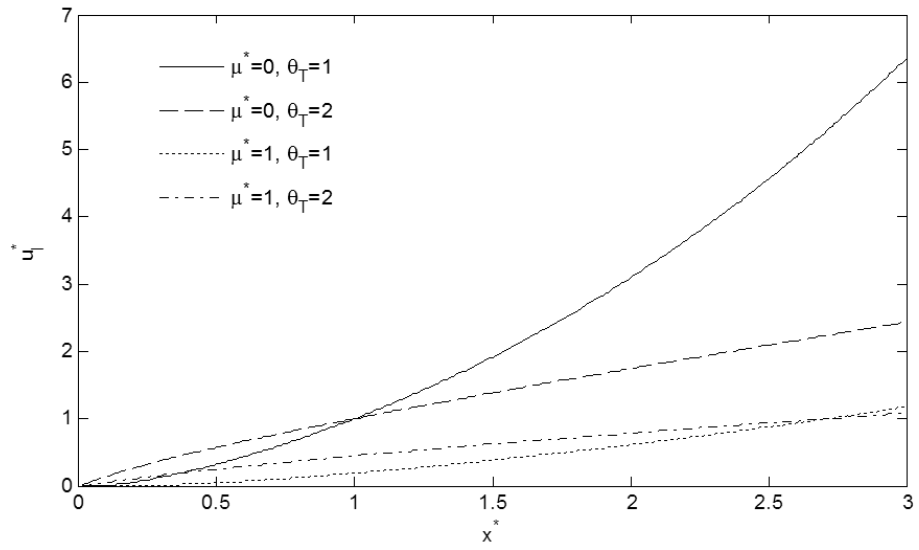

Fig 4.5. Variations of $u_{l}^{*}$ with dimensionless location

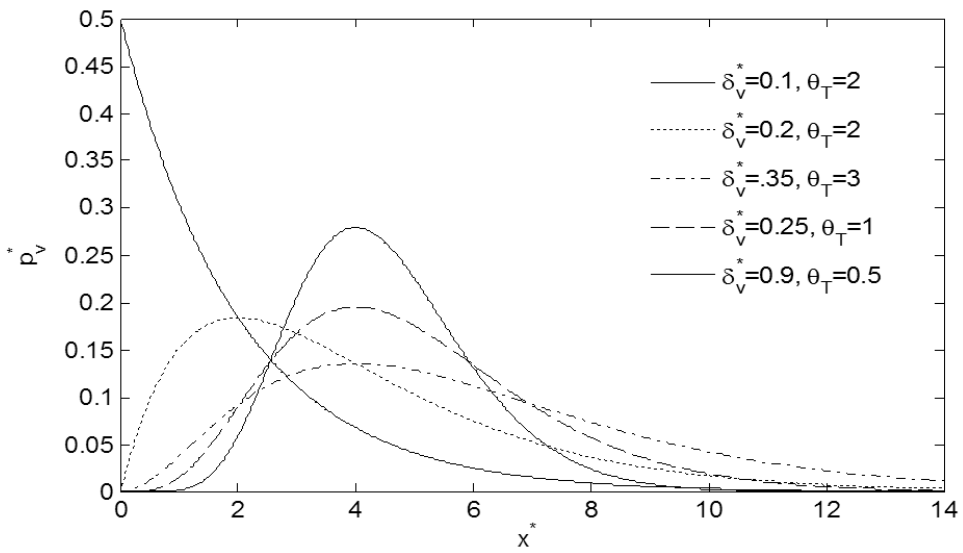

Fig 4.6. Variations of $p_{v}^{*}$ with dimensionless location

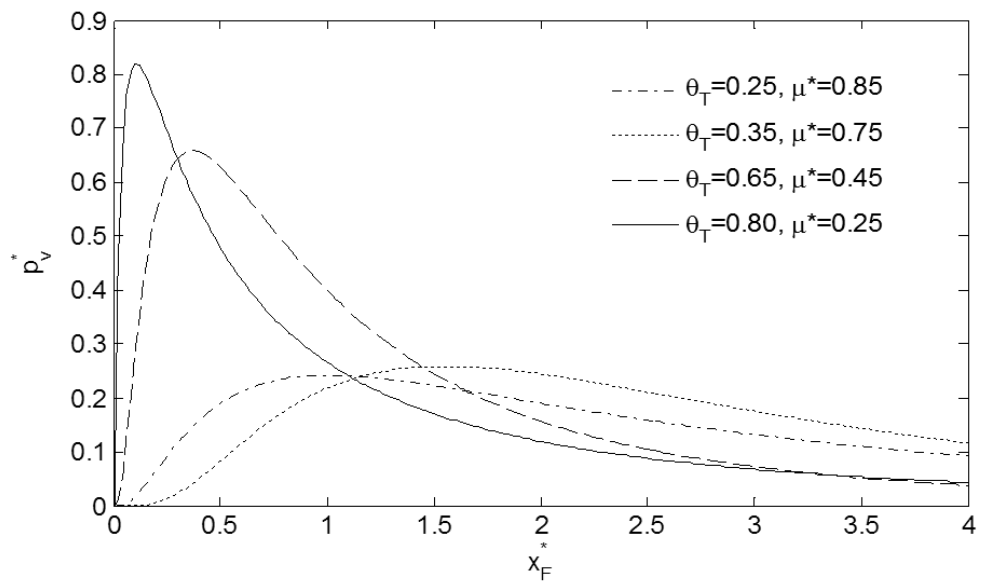

Fig 4.7. Variations of $p_{v}^{*}$ with dimensionless front location

Figure 4.8 shows variations of $\theta_{T}$ with dimensionless front location and Fig 4.9 shows the profiles for dimensionless fluid temperature, pressure, saturation, and the velocity of the 
vapor phase over the whole domain of the porous medium. Due to the great temperature difference between solid and fluid phase, condensation occurs. Most of the condensed water is moved downstream the condensation front into the condensate region which as a consequence continuously grows in size. Temperature and pressure of the fluid phase are coupled in the whole domain and, therefore, show similar profiles. The increase of the vapor velocity results in a higher pressure gradient and since pressure and temperature are coupled, a decrease of fluid temperature and pressure occurs. The acceleration of the vapor phase towards the condensation front is due to the phase change of the vapor. The saturation profiles show the progress of the condensation front. Further worthy of attention is the fact that the pressure loss in the condensate region $(s=1)$ can be neglected compared with the high pressure gradient in the vapor phase region.

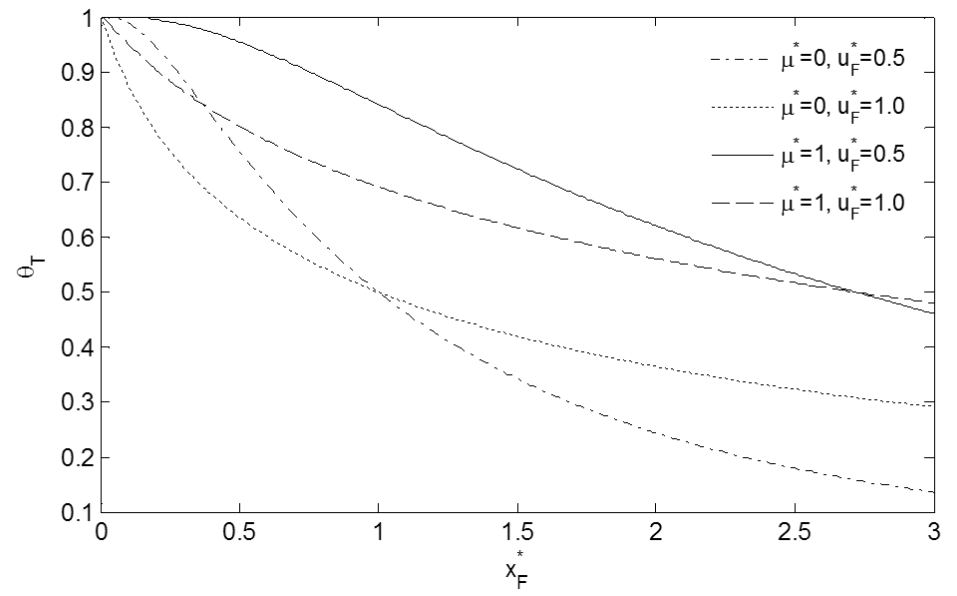

Fig 4.8. Variations of $\theta_{T}$ with dimensionless front location 


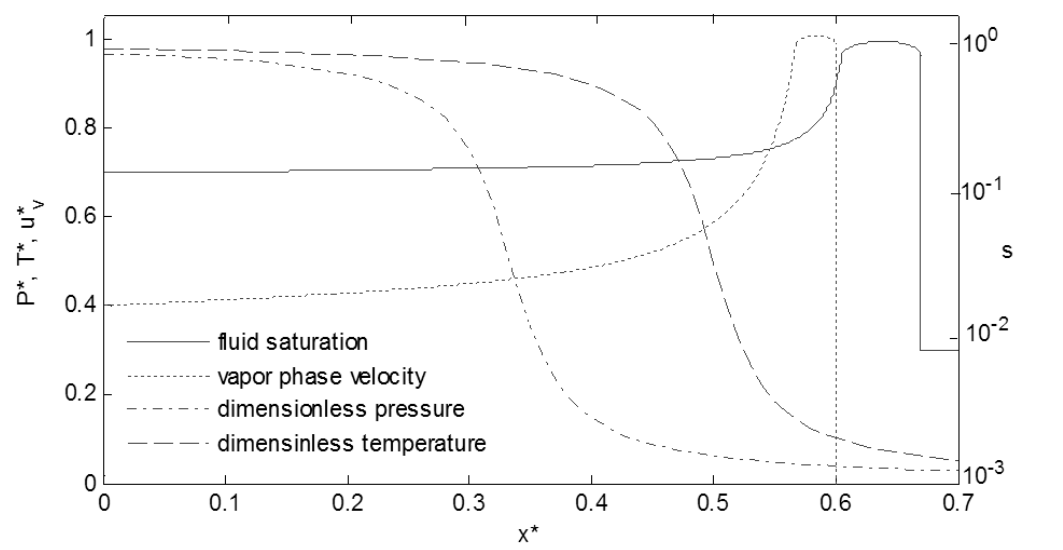

Fig 4.9. Profiles for fluid temperature, pressure, saturation, and the velocity of the vapor phase

\subsection{SUMMARY}

The condensation-front speed and liquid-phase distribution in the condensate region have been examined theoretically. Three different regions and a condensation zone are defined. The dimensionless vapor parameters of the problems are $J a, B o, p_{0}^{*}$, and the thermo physical properties. The dimensionless front speed $u_{F}^{*}$ or the front location $x_{F}^{*}$ are found for the down flow of vapor in the porous medium. For vapor flow, a liquid region is present and increases in thickness with the distance from the inlet of vapor. The ratio of condensation front velocity to bond number $u_{F}^{*} / B o$ changes with respect to $x_{F}^{*} J a / p_{0}^{*}$ and $u_{V}^{*} / B o$ is a function of $x_{F}^{*} J a / p_{0}^{*}$. 


\section{CHAPTER FIVE: VAPOR CONDENSATION HEAT TRANSFER IN POROUS MEDUM}

\subsection{INTRODUCTION}

The fluid and heat processes occurring in porous medium or wick are different from those occurring in conventional channels so that some parameters are introduced to characterize the structure and properties of porous wick. Among them, the permeability characterizes mass transfer in porous wick. The porosity, effective pore radius, and permeability are a set of parameters which are only related to the porous wick. Udell [45], for example, studied the effects of porosity, effective pore radius, particle size, permeability, evaporation, condensation and capillary on a heated from above porous media's performance. Figus et al. [46] developed two two-dimensional mathematical models separately based on the Darcy model and the pore network model, both of which considered the moving liquid vapor interface in the wick to study steady heat transfer in the wick with a single or varying pore size distribution. They concluded that the two models agreed and produced similar results. Cao and Faghri [47] developed twodimensional mathematical model for heat transfer in the capillary wick and the cover plate with three-dimensional vapor flow in the groove. Based on the Laplace-type equation Cao and Faghri [48] obtained the two-dimensional analytical solutions of flow and heat transfer in the capillary wick for pressure and the perturbation method for temperature. Zhao and Liao [49] investigated experimentally heat transfer in packed cubic porous media with a groovy heated wall on the top. Liao and Zhao [50] investigated experimentally the effects of particle size, inlet temperature, and adverse hydrostatic head on the total heat transfer in the case of evaporative heat transfer in a capillary structure heated by a grooved block. By using the Green's function method, LaClair and Mudawar [51] developed a one dimensional approximate solution for the coupled liquid core, capillary wick, and metal shell. They considered the capillary wick as a fully liquid- 
saturated wick with the liquid vapor interface fixed at the surface of the wick. Demidov and Yatsenko [52] developed a two-dimensional mathematical model considering both convection and the free liquid-vapor interface in the wick, and investigated numerically steady capillarydriven flow and heat transfer in rectangular porous media. Khrustalev and Faghri [53] developed a one-dimensional mathematical model on the basis of heat transfer with thin-film evaporation theory in a pore and heat transfer in the dry porous media and close-touched heated fins, and the analytical steady state solution was derived. The porous wick provides the capillary pressure to drive the two-phase flow, and supplies the passages for liquid flow and evaporation. Muraoka et al $[54,55]$ showed an effective way to increase the performance is by reducing the pressure drop in the evaporator. In order for them to create a fixed stable physical interface, a conventional tube condenser was replaced by a porous wick. It was concluded from their experimental and theoretical studies that the existence of a porous wick could reduce or even eliminate the difficulties associated with the start-up procedure and the occurrence of the pressure oscillation during operation. Kaya and Hoang [56] used a primary and a secondary wick to ensure that liquid stay in the wicks all the time.

Most recently, various kinds of porous wicks have been employed, such as nickel [57], copper [58], titanium [59], and ceramic [60] wicks. Among these types, the titanium porous wicks exhibit good mechanical properties for the processing of the vapor grooves, and the excellent compatibility to different working liquid. Therefore, they have been widely used in vapor condensation heat exchanger devices.

A summary of the efforts in the mathematical description of heat and mass transfer in capillary-porous media has been given by Luikov [61] and Ogniewicz and Tien [62]. The insight into the permeability and flow properties of porous wick are still limited. Therefore, more 
detailed investigations are required to characterize the permeability as well as capillary pressure of the porous wicks for the design of vapor condensation heat exchangers. Hence, this issue should be addressed to employ the capillary process to assess the capillary performance of wicks. Despite difficulties to determine capillary pressure and permeability, in recent years, capillary experiments coupled with analysis method have been found to be able to determine these two properties of a porous wick [63-64]. Infrared (IR) thermal imaging method was developed by Tang et al [63] and Deng et al [64] to accurately monitor the capillary processes in composite wicks combined with microgrooves. The capillary performance of composite wicks, which integrates both capillary pressure and permeability, was assessed. Crone et al [65] presented a numerical model describing condensation flow in porous media including the influence of porous parameters on heat transfer and process kinetics. Bergins et al [66] introduced a modeling approach which includes a mathematical solution to describe the proceeding of a flat condensation in a porous medium during heating with saturated steam. They compared their model with experimental results and calculations of the numerical model of Crone et al [65] and found good agreement. Comparison of their results also showed an excellent agreement with experimental data of Brouwers [67] in a wide range of steam pressures and permeabilities.

Therefore, capillary pressure and permeability of the porous wick are two key parameters to determine the heat transfer performance. For the purpose of working efficiently, the porous wick should meet two competing demands, i.e., large capillary pressure which needs a small pore radius and high permeability which requires a large pore type. Therefore, it is critical to assess these two parameters for the good design of porous wicks. The porous wick's performance is influenced by many factors. They are classified into three kinds: 1) the outer parameters, such as inlet pressure, inlet temperature, and outlet pressure; 2) the parameters related to the porous 
wick, such as porosity, effective pore radius, permeability; 3) properties of the working fluid, such as viscosity and latent heat of evaporation. The permeability is generally treated as the function of porosity and effective pore radius. In this chapter, the focus is on the effect of the permeability on the wick's performances. The change of the permeability can be characterized by changing porosity or effective pore radius, respectively. The porosity, effective pore radius, and permeability are correlative parameters. The effect of permeability associated with porosity and effective pore radius on a wick's performances is investigated and the condensation flow movement in terms of time of porous wick will be depicted in this chapter.

\subsection{GOVERNING EQUATIONS}

The governing equations that will be used in this chapter are similar to governing equations used in the previous chapter. The calculation of the momentum, mass and energy balances in an integral form was done in the previous chapter and it was to make sure that the basic conservation principles were satisfied on a macroscopic scale. The flow was divided into three different regions. In the first region, the vapor flowed to the condensation forepart where it condensed. The resulting condensate flowed off and replaced the vapor that initially filled the void space of the porous wick. The condensation took place only at the condensation forepart and the condensate flowed at full saturation $s=1$ and behaved according to Darcy's law. It was also assumed in the previous chapter that the condensed working liquid replaced the vapor at a velocity which was calculated from Darcy's law due to the low speed of the condensation forepart compared to the velocity of vapor. In this chapter the same approach is used and the goal is to find an expression $Z_{v}(t)$ or $t\left(Z_{v}\right)$ for the relationship between the length of the vapor

condensation flow $L-Z_{v}-\Delta Z_{i}$ and the respective time $t$. Assuming a quasi steady state 
behavior of the porous heating process, the sum of the pressure losses in the different regions is equal to the external pressure gradient,

$$
\sum \Delta p=\left(p_{0}-p_{1}\right)+\Delta p_{l}+\Delta p_{v}+\Delta p_{i}+\Delta p_{c}
$$

Thermal energy should also be conserved within the porous wick. For the momentum in steady state, Darcy-Ergun momentum relation $[68,69]$ has to be applied for liquid pressure drop which contribute to liquid phase transport in two-phase regions as well as the modified thermodynamic equilibrium conditions for the liquid-vapor system. Thus the modified vapor and liquid relative permeabilities expressions were introduced by Wyllie [68],

$$
-p_{v} \frac{d p_{v}}{d z}=p_{0}\left(\frac{\mu_{v}}{K K_{v, r}} u_{v, D}+\frac{\rho_{v}}{K^{*} K_{v, r}^{*}} u_{v, D}^{2}\right)
$$

where $K^{*}$ is introduced to take into account the reduction of cross section area and therefore the increase in pressure loss. Also, $\left(\frac{\mu_{v}}{K K_{v, r}} u_{v, D}+\frac{\rho_{v}}{K^{*} K_{v, r}^{*}} u_{v, D}^{2}\right)$ does not depend on $z$ and $p$. Rearranging Eq. (176),

$$
-p_{v} d p_{v}=p_{0}\left(\frac{\mu_{v}}{K K_{v, r}} u_{v, D}+\frac{\rho_{v}}{K^{*} K_{v, r}^{*}} u_{v, D}^{2}\right) d z
$$

Integrating Eq. (177) results in

$$
-\frac{1}{2} p_{v}(z)^{2}=p_{0}\left(\frac{\mu_{v}}{K K_{v, r}} u_{v, D}+\frac{\rho_{v}}{K^{*} K_{v, r}^{*}} u_{v, D}^{2}\right) z+\text { Const } .
$$

the constant in Eq. (178) can be obtained by setting $z=0$ i.e.,

$$
-\frac{1}{2} p_{v}(0)^{2}=\text { Const }
$$

the Darcy velocity $u_{v, D}$ can be calculated from the pore velocity $u_{v}$ by 


$$
u_{v}=\frac{u_{v, D}}{\varepsilon_{v}}=\frac{u_{v, D}}{\varepsilon(1-s)}
$$

Combining Eq. (178) and Eq. (179) results in

$$
\frac{1}{2}\left(p_{v}(0)^{2}-p_{v}(z)^{2}\right)=p_{0}\left(\frac{\mu_{v}}{K K_{v, r}} u_{v, D}+\frac{\rho_{v}}{K^{*} K_{v, r}^{*}} u_{v, D}^{2}\right) z
$$

Rearranging and assuming ideal gas behavior, Eq. (181) can be expressed as

$$
\frac{1}{2} \frac{p_{v}(0)^{2}-p_{v}(z)^{2}}{p_{0} z}=\frac{\bar{\mu}_{v, \mathrm{~S}}}{K_{v}} u_{v, D .0}+\frac{\rho_{v, 0}}{K_{v}^{*}} u_{v, D .0}^{2}
$$

By knowing that $\left(\frac{\bar{\mu}_{v, S}}{K_{v}} u_{v, D .0}+\frac{\rho_{v, 0}}{K_{v}^{*}} u_{v, D .0}^{2}\right)$ does not depend on $z$, conformation of left term of Eq.

(182) with $Z$ and $Z_{v}$ can be expressed as

$$
\frac{1}{2} \frac{p_{v}(0)^{2}-p_{v}(z)^{2}}{p_{0} z}=\frac{1}{2} \frac{p_{v}(0)^{2}-p_{v}\left(Z_{v}\right)^{2}}{p_{0} Z_{v}}
$$

and rearranging Eq. (183)

$$
p_{v}(z)^{2}-p_{v}(0)^{2}=-\frac{z}{Z_{v}}\left(p_{v}(0)^{2}-p_{v}\left(Z_{v}\right)^{2}\right)
$$

the pressure distribution in the vapor region can be expressed as

$$
p_{v}(z)=\sqrt{p_{v}(0)^{2}-\frac{z}{Z_{v}}\left(p_{v}(0)^{2}-p_{v}\left(Z_{v}\right)^{2}\right)}
$$

If the mean viscosity $\bar{\mu}_{v, s}$ is calculated by

$$
\bar{\mu}_{v, s}=\frac{1}{L} \int_{0}^{L} \mu_{v, s}\left(T_{s}(p(z))\right) d z
$$


The velocities of the condensed liquid $(I)$ in region (III) are small compared with the vapor velocity in region $(I)$. Therefore Darcy's law is sufficient to describe the pressure loss in these regions i.e.,

$$
\begin{gathered}
\Delta p_{l}=\frac{\mu_{l}}{K_{l}} \varepsilon \mu_{l} \Delta Z_{l} \\
\Delta p_{i}=\frac{\mu_{i}}{K_{i}} \varepsilon \mu_{i} \Delta Z_{i}
\end{gathered}
$$

the macroscopic capillary pressure, which appears in is generated by the liquid-vapor interface and the surface tension of liquid, which is known as the Laplace-Young equation i.e.,

$$
\Delta P_{c, \text { mac }}=\frac{2 \sigma \cos \theta}{r_{p}}
$$

where $\sigma$ is the surface tension of liquid, $\theta$ is contact angle formed between solid and liquid, and $r_{p}$ is the pore radius, which is often characterized as the mean pore radius. The pore radius and the contact angle cosine are generally replaced by effective pore radius $r_{\text {eff }}$, and Eq. (189) can be expressed as

$$
\Delta P_{c, \text { mac }}=\frac{2 \sigma}{r_{\text {eff }}}
$$

the microscopic capillary pressure that exists in the meniscii inside the pores of the porous wick is to be considered. For a point of contact of a meniscus with a sphere as indicated in fig. $2 b$, the differential force $d f$ that acts vertically on a segment $d p$ of the contact line satisfies

$$
\frac{d f}{d p}=\gamma \cos \alpha=\gamma \sin (\phi+\alpha)
$$


For any array of spheres the meniscii shapes can be very complex. However, it is expected that Eq. (191) holds for any point of the contact line. The total force acting on the meniscus limited by the region inside the sphere can be calculated from the contact line perimeter, giving

$$
f=2 \pi R \gamma \sin \phi \sin (\phi+\theta)
$$

Eq. (192) is similar to the relation for an isolated sphere. On the other hand, the projected area (A) of the meniscus inside that region is

$$
A=\left(\frac{4}{\pi \sin ^{2} \phi}-1\right) \pi R^{2} \sin ^{2} \phi
$$

The microscopic capillary pressure, $\Delta p_{c, m i c}$, which in this case is representative of the whole interface, is found as the ratio of the total vertical force, Eq. (192), and the projected area, Eq. (193), of the meniscus,

$$
\Delta P_{c, \text { mic }}=\frac{2}{\frac{4}{\pi \sin ^{2} \phi}-1} \frac{\sin (\phi+\theta)}{\sin \phi} \frac{\gamma}{R}
$$

the capillary pressure changes with the height of the contact point of the meniscus, $h_{c}$, given by

$$
h_{c}=R(1-\cos \phi)
$$

For the porous wick used here, it is not possible to reach the values $h_{c}=0$ and $h_{c}=2 R$, since the meniscus position is limited by the spheres located above and below it. Using only the points of contact with the spheres it can be shown that $\phi$ is limited by the values $\phi^{\min }=0.25 \pi$ and $\phi^{\max }=0.75 \pi . \Delta p_{c, \text { mic }}$ values for the limit values of $\phi$ can be obtained using Eq. (194). When $\theta=0, \Delta p_{c}$ is at its optimal value at $h_{c}=R$, which corresponds to $\theta=\pi / 2$, thus when $\theta=0$, the 
minimum value of the capillary pressure is found just before touching the upper sphere, in the position given by $\phi^{\max }=0.75 \pi$. Using Eq. (194),

$$
\Delta P_{c, m i c}=\left(\frac{2}{\frac{8}{\pi}-1}\right) \frac{\gamma}{R}
$$

The total pressure loss is the sum of the pressure losses of vapor, liquid, air and capillary. Due to the complexity of the analytical solution, the detailed derivation is given in Appendix A, Eqs. (A.1)-(A.10), so the total pressure loss i.e.,

$$
\begin{aligned}
\Delta p=\left(p_{v}(0)-p_{v}\left(Z_{v}\right)\right)+ & \Delta p_{l}+\Delta p_{i}+\Delta p_{c}=p_{v}(0)-p_{v}(0) \sqrt{1-2 \frac{\varepsilon_{v} \mu_{v, 0}}{p_{0}}\left(\frac{\mu_{v, \mathrm{~s}}}{K_{v}}+\frac{\rho_{v, 0}}{K_{v}^{*}} \varepsilon_{v} \mu_{v, 0}\right) Z_{v}} \\
& +\frac{\mu_{l}}{K_{l}} \varepsilon \mu_{l} \Delta Z_{l}+\frac{\mu_{i}}{K_{i}} \varepsilon \mu_{i} \Delta Z_{i}+\frac{2 \sigma}{r_{\text {eff }}}+\frac{2 \gamma}{R\left(\frac{8}{\pi}-1\right)}
\end{aligned}
$$

It is assumed that the vapor flow extends over a length $Z_{v}$ up to the condensate forepart (condensation front). In this region the liquid is assumed to be quiescent, the saturation $s$ is constant $\left(s=s_{q}\right)$ and only minor condensation takes place in the porous wick. Until the condensed flow reaches the exit of the porous wick, the length of the gas (vapor) region is given as

$$
\Delta Z_{i}=L-Z_{v}-\Delta Z_{l}
$$

The macroscopic energy balance for the heating of porous wick of length $Z_{v}$, corresponding to the length of the condensation, can be written as

$$
m_{v}\left(h_{v, 0}-h_{l, 1}\right)=A Z_{v}\left(\rho c_{p}\right) \beta_{\text {loss }}\left(\bar{T}_{s}-T_{1}\right)
$$


with $A$ being the cross-sectional area of the porous wick, $T_{1}$ is the initial(ambient) temperature and $m_{v}$ is the mass flow of vapor. The vapor condenses and is cooled to the initial temperature of the bulk. Therefore the difference between the enthalpy of the vapor at the entrance $h_{v, 0}$ and respective value of the condensed liquid at ambient temperature $h_{l, 1}$ can be used for the vaporizing. The correction factor $\beta_{\text {loss }}$ describes heat losses from the radiation of the setup and heating of insulation material. The mean temperature $\bar{T}_{s}$ of saturated vapor, residual liquid and solid in the vaporized region is calculated from

$$
\bar{T}_{s}=\frac{1}{L} \int_{0}^{L} T_{v, s}\left(p_{v}(z)\right) d x
$$

using the pressure distribution $p_{v}(z)$ which results from the flow through the porous wick of length $L$ according to Eq. (185) with $Z_{v}=L$. The mean volumetric heat capacity $\left(\rho c_{p}\right)$ of vapor and liquid phases in region $(I)$ is calculated according to the phase distribution of solid material $(s)$, residual liquid $(l)$ and vapor $(v)$ as

$$
\left(\rho c_{p}\right)=(1-\varepsilon) \rho_{s} c_{p, s} \beta_{w m}=\varepsilon s_{q} \rho_{l, s} c_{p, l, s}+\varepsilon_{v} \rho_{v, s} c_{p, v, s}
$$

with $\varepsilon v=\varepsilon\left(1-s_{q}\right) .(s)$ denotes the saturated state of liquid and vapor. The introduction of the factor $\beta_{w m}$ enables the consideration of an additional heating of the porous wick material(Volume $V_{w m}$, density $\rho_{w m}$, heat capacity $c_{p, w m}$, length $L$ ). With respect to the heating of the solid itself, $\beta_{w m}$ is defined as follows 


$$
\beta_{w m}=1+\frac{V_{w m} \rho_{w m} c_{p, w m}}{A L(1-\varepsilon) \rho_{S} c_{p, s}}
$$

From the energy balance in Eq. (199) the vapor mass flow $m_{v}$ and the vapor mass flow rate $d m_{v} / d t$ which is also related to the pore velocity $u_{v, 0}$ of the vapor can be written as a function of cross-sectional area $A$, vapor region length $Z_{v}$ and energy balance constant $\xi$ i.e.,

$$
\begin{gathered}
m_{v}=A \frac{\left(\rho c_{p}\right) \beta_{\text {loss }}\left(\bar{T}_{s}-T_{1}\right)}{h_{v, 0}-h_{l, 1}} X_{v}=A \xi Z_{v} \\
\frac{d m_{v}}{d t}=A \xi \frac{d Z_{v}}{d t}=\varepsilon_{v} u_{v, 0} \rho_{v, 0} A
\end{gathered}
$$

The mass balance takes into account the residual liquid, the vapor suspension and the liquid in the condensation front region (III)

$$
\left(m_{v}\right)=A\left(Z_{v} \varepsilon s_{q} \rho_{l, s}+Z_{v} \varepsilon_{v} \rho_{v, s}+\Delta Z_{l} \varepsilon \rho_{l}\right)
$$

Now the width of the condensation region $\Delta Z_{l}$ can be calculated from Eqs. (203) and (205)

$$
\Delta Z_{l}=Z_{v} \frac{\xi-\varepsilon s_{q} \rho_{l, s}-\varepsilon\left(1-s_{q}\right) \rho_{v, s}}{\varepsilon \rho_{l}} Z_{v}=\zeta Z_{v}
$$

Eq. (206) also gives the condition $\Delta Z_{l} / Z_{v}>0$, which is necessary for the formation of a fully saturated condensed region. If this condition is not valid, the mass balance constant $\zeta$ is set equal to $\mathbf{O}$.The pore velocity of the vapor can be calculated from the energy balance from Eq. (204)

$$
u_{v, 0}=\frac{d Z_{v}}{d t}\left(\frac{\xi}{\varepsilon_{v} \rho_{v, 0}}\right)
$$


The pore velocities of liquid and vapor phase are the sum of condensation front speed and the speed of condensed region growth, expressed by the mass balance from Eq. (206)

$$
u_{l}=u_{i}=\frac{d Z_{v}}{d t}+\frac{d \Delta Z_{l}}{d t}=\frac{d Z_{v}}{d t}(1+\zeta)
$$

Eq. (197) is for times before liquid become stagnant $\left(Z_{v}+\Delta Z_{l} \leq L\right)$ and can be written as

$$
\begin{aligned}
\Delta p= & p_{v}(0)-p_{v}(0) \sqrt{1-2 \frac{\xi^{2}}{\rho_{v, 0} p_{0} K_{v}^{*}}\left(\frac{\mu_{v, s} K_{v}^{*}}{\xi K_{v}}+\frac{d Z_{v}}{d t}\right) \frac{d Z_{v}}{d t} Z_{v}} \\
& +\left[\frac{\mu_{l}}{K_{l}} \varepsilon \zeta Z_{v}+\frac{\mu_{i}}{K_{i}} \varepsilon\left(L-Z_{v}(\zeta+1)\right)\right](\zeta+1) \frac{d Z_{v}}{d t}+\frac{2 \sigma}{r_{e f f}}+\frac{2 \gamma}{R\left(\frac{8}{\pi}-1\right)}
\end{aligned}
$$

with Eqs (206)-(208) and (198). After the condensate region has reached the exit, using $\left(\Delta Z_{i}=0\right)$

and $\left(\Delta Z_{l}=L-Z_{v}\right)$ Eq. (197)can be written as

$$
\Delta p=p_{v}(0)-p_{v}(0) \sqrt{1-2 \frac{\xi^{2}}{\rho_{v, 0} p_{0} K_{v}^{*}}\left(\frac{\mu_{v, \mathrm{~s}} K_{v}^{*}}{\xi K_{v}}+\frac{d Z_{v}}{d t}\right) \frac{d Z_{v}}{d t} Z_{v}}+\left[\frac{\mu_{l}}{K_{l}} \varepsilon(\zeta+1)\left(L-Z_{v}\right)\right] \frac{d Z_{v}}{d t}+\frac{2 \sigma}{r_{e f f}}+\frac{2 \gamma}{R\left(\frac{8}{\pi}-1\right)}
$$

\subsection{RESULTS\& DISCUSSIONS}

Equations (209) and (210) represent the governing differential equations, which can be solved analytically to derive an expression $Z_{v}(t)$ or $t\left(Z_{v}\right)$ for the relation between the length of the vapor flow $Z_{v}$ and the respective time $t$. Due to the complexity of the analytical solution the detailed derivation is given in Appendix A. As a result of the mathematical operations given in Appendix A, the analytical solution of Eqs. (209) and (210) is expressed as 


$$
t=\frac{1}{2 C_{6}}\left(\begin{array}{l}
\frac{1}{2} C_{7} Z_{v}^{2}+C_{8} Z_{v}+\frac{1}{2}\left(Z_{v}+\frac{1}{2} \frac{C_{10}}{C_{9}}\right) \sqrt{C_{9} Z_{v}^{2}+C_{10} Z_{v}+C_{11}} \\
+C_{12} \ln \left[\sqrt{C_{9}}\left(Z_{v}+\frac{1}{2} \frac{C_{10}}{C_{9}}\right)+\sqrt{C_{9} Z_{v}^{2}+C_{10} Z_{v}+C_{11}}\right]+\frac{2 \sigma}{r_{e f f}}+\frac{2 \gamma}{\frac{8 R}{\pi}-R}
\end{array}\right)+\text { const. }
$$

with

$$
C_{12}=\frac{1}{8} \sqrt{C_{9}}\left[4 \frac{C_{11}}{C_{9}}-\left(\frac{C_{10}}{C_{9}}\right)^{2}\right]
$$

the analytical solution is used for the further calculations and the discussion of the physical phenomena of vapor flow and condensation processes. The constant of the integration can be calculated from the initial conditions for the time before liquid become stagnant

$$
Z_{v}(t=0)=0
$$

and after liquid become stagnant

$$
Z_{v}\left(t_{l b s}\right)=\frac{L}{1+\zeta}
$$

If the pressure loss of the vapor phase is considered in Eq. (197), the mass and heat balances are simplified and the resulting differential equation can be expressed as

$$
\frac{1}{2} \frac{p_{v}(0)^{2}-p_{l}(0)^{2}}{p_{0}} \frac{\rho_{v, 0} K_{v}}{\xi \bar{\mu}_{v, s}}=\frac{d Z_{v}}{d t} Z_{v}+\frac{K_{v} \xi}{K_{v}^{*} \bar{\mu}_{v, s}}\left(\frac{d Z_{v}}{d t}\right)^{2} Z_{v}+\frac{2 \sigma}{r_{e f f}}+\frac{2 \gamma}{\frac{8 R}{\pi}-R}
$$

with $p_{v}\left(Z_{v}\right)=p_{1}$ since the pressure loss of liquid and air is neglected. Integration of Eq. (176)

using a mean constant density $\bar{\rho}_{v, s}$ and inserting Eq. (207) results in the differential equation

$$
\left(p_{0}-p_{1}\right) \frac{\rho_{v, 0} K_{v}}{\xi \bar{\mu}_{v, s}}=\frac{d Z_{v}}{d t} Z_{v}+\frac{K_{v} \xi}{K_{v}^{*} \bar{\mu}_{v, s}} \frac{\bar{\rho}_{v, s}}{\rho_{v, 0}}\left(\frac{d Z_{v}}{d t}\right)^{2} Z_{v}
$$

Both equations are similar to 


$$
C_{13}=\frac{d Z_{v}}{d t} Z_{v}+C_{14}\left(\frac{d Z_{v}}{d t}\right)^{2} Z_{v}+\frac{2 \sigma}{r_{e f f}}+\frac{2 \gamma}{\frac{8 R}{\pi}-R}
$$

with

$$
C_{13}=\frac{1}{2} \frac{p_{0}^{2}-p_{1}^{2}}{p_{0}} \frac{\rho_{v, 0} K_{v}}{\xi \mu_{v, s}}, \quad C_{14}=\frac{K_{v} \xi}{K_{v}^{*} \bar{\mu}_{v, s}}
$$

or

$$
C_{13}=\left(p_{0}-p_{1}\right) \frac{\rho_{v, 0} K_{v}}{\xi \mu_{v, s}}, \quad C_{14}=\frac{K_{v} \xi}{K_{v}^{*} \bar{\mu}_{v, s}} \frac{\bar{\rho}_{v, \mathrm{~s}}}{\rho_{v, 0}}
$$

Eq. (217) can be solved similar to Eq.(A.47) with the substitution procedure described in the Appendix A. With the initial condition $Z_{v}(t=0)=0$ the solution is given as

$$
t=\frac{1}{2 C_{13}}\left(\begin{array}{l}
\frac{1}{2} Z_{v}^{2}+\frac{1}{2}\left(Z_{v}+\frac{1}{2}\left(4 C_{13} C_{14}\right)\right) \sqrt{Z_{v}^{2}+\left(4 C_{13} C_{14}\right) Z_{v}} \\
-\frac{1}{8}\left(4 C_{13} C_{14}\right)^{2} \ln \left[Z_{v}+\frac{1}{2}\left(4 C_{13} C_{14}\right)+\sqrt{Z_{v}^{2}+\left(4 C_{13} C_{14}\right) Z_{v}}\right] \\
+\frac{1}{8}\left(4 C_{13} C_{14}\right)^{2} \ln \left[\frac{1}{2}\left(4 C_{13} C_{14}\right)\right]+\frac{2 \sigma}{r_{\text {eff }}}+\frac{2 \gamma}{\frac{8 R}{\pi}-R}
\end{array}\right)
$$

For the description of vapor flow with small velocities during the whole condensation process $C_{14} \rightarrow 0$, consequently the solution in Eq. (220) can be simplified further,

$$
t=\frac{1}{2 C_{13}} Z_{v}^{2}+\frac{2 \gamma}{\frac{8 R}{\pi}-R} \text { or } Z_{v}=\sqrt{2 C_{13} t}+\frac{2 \sigma}{r_{\text {eff }}}+\frac{2 \gamma}{\frac{8 R}{\pi}-R}
$$

Eq. (221) is the integral solution of the momentum balance in Eqs. (215) and (216) without the quadratic term. It only describes the movement of a condensation front with small velocities, so 
an estimation for the beginning of the vapor flow can be done by neglecting the viscous linear term of the momentum balance

$$
Z_{v}=\left(\frac{3}{2} \sqrt{\frac{C_{13}}{C_{14}} t}\right)^{2 / 3}+\frac{2 \sigma}{r_{e f f}}+\frac{2 \gamma}{\frac{8 R}{\pi}-R}
$$

Besides Eqs. (221) and (222) a third case can be calculated for the vaporization of an initially saturated porous wick during which the pressure loss of the liquid dominates the kinetic of the process. Neglecting the pressure loss of the liquid phase the integration of Eq. (210) results in

$$
t=C\left(L Z_{v}-\frac{1}{2} Z_{v}^{2}\right)+\frac{2 \sigma}{r_{e f f}}+\frac{2 \gamma}{\frac{8 R}{\pi}-R}
$$

Eq. (223) implements that the length $Z_{v}$ is proportional to time $t$ for small values of $Z_{v}$.

Figure 5. 1. (a-b) shows condensation flow length in terms of time for the four kinds of titanium and nickel wicks using the two working fluids, wet (water vapor) steam and dry steam. The data in these plots represents capillary and non capillary effects for each wick material during each fluid test. When the wick materials contacted with the liquid, the meniscus firstly propagated rapidly in the porous wick, as at this time the differential force $d f$ that acts vertically on a segment $d p$ of the contact line was small according to Eq. (191). By reaching the condensation front the vapor flow movement tended to become smaller and the capillary capability increased steadily. The capillary process in the porous materials can sustain, as the tiny pores in the porous wick provided good capillary pressure. 

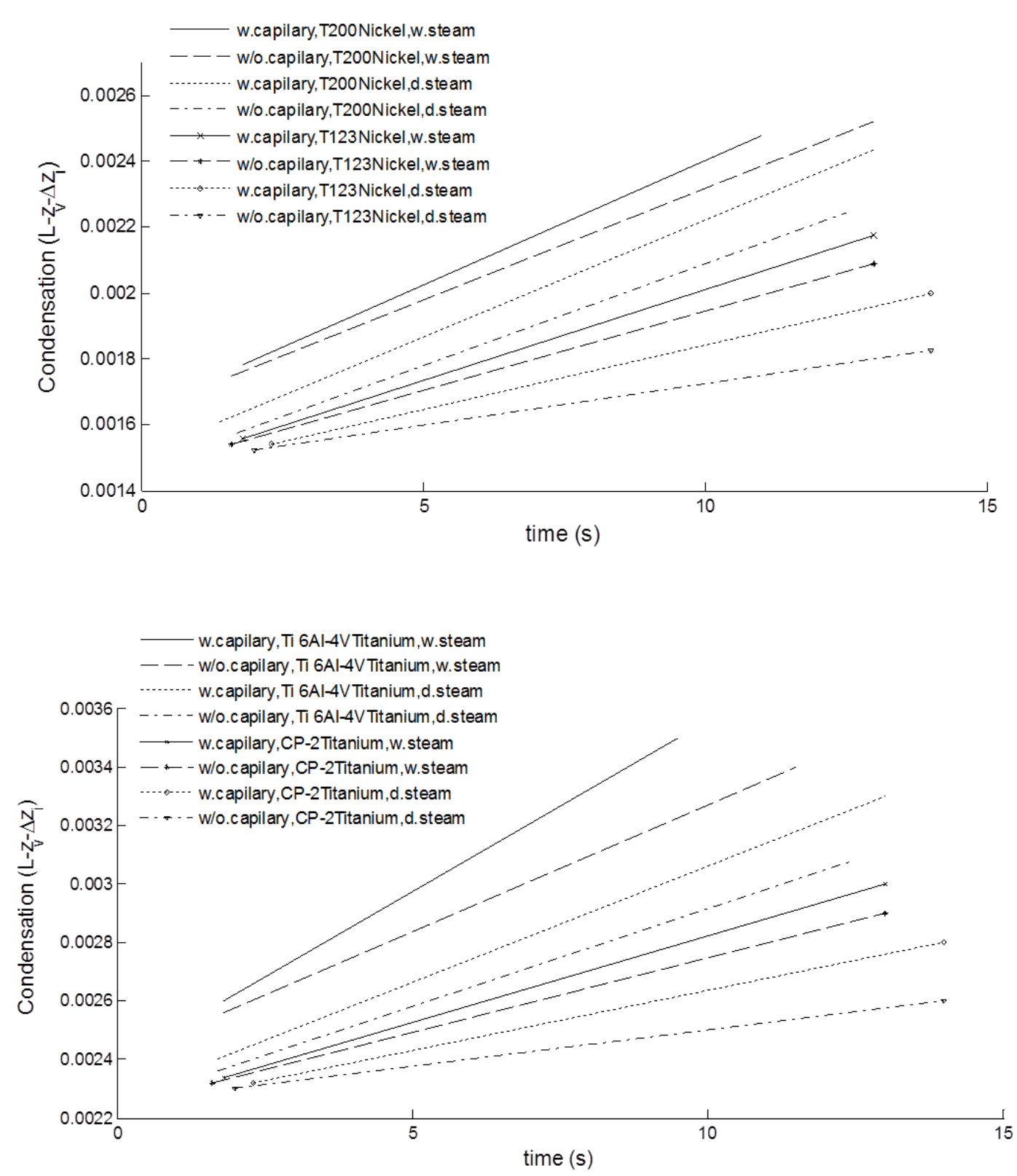

Figure 5.1. Condensation flow length in terms of time for the four kinds of titanium and nickel wicks with two working fluids, wet (water vapor) steam and dry steam.

The titanium porous wick exhibits a larger condensation flow length thus larger movement than the nickel porous wick. With the time going on, the difference of condensation length between titanium and nickel porous materials became distinctive, in which the material of Ti 6Al-4V and Ni T123 presents the highest and lowest behavior, respectively. The different condensation movement for these porous wick materials suggests a different capillary 
performance for these four porous wick materials. From table. 5.1, the $\Delta P_{c} \cdot K$ of both titanium wicks were much larger than those of the nickel ones. Despite that the porosities between titanium and nickel wicks were slightly different, the significantly different pore size formed in titanium and nickel porous wicks played the dominant role in the capillary performance. The particle sizes of nickel were much smaller than those of titanium materials.

The tiny pore in nickel porous wicks tended to form with an order of $2.25-9.75 \mu \mathrm{m}$. This induces an order of $10^{13} \mathrm{~mm}^{2}$ of permeability for the nickel porous wicks, which is one order of magnitude lower than the titanium porous wicks, as shown in table 5.1. Though the capillary pressure of nickel wicks was larger than the titanium ones as predicted by Eq. (190), the lower permeability of nickel wicks offset the capillary pressure, thereby inducing a smaller capillary performance as compared to the titanium wicks. When comparing two kinds of Nickel wicks, T123 and T200, it can be seen that Ni T200 produced a larger condensation length, as shown in figure 5.1. (a-b), which is true for both fluids test. The capillary performance parameter $\Delta P_{c} \cdot K$ of T200 was also found to be superior to that of T123. Though the particle size of T123 was larger than the T200, the pore size of the former porous wick was not necessarily larger than the latter.

For the comparison of two kinds of Titanium wicks, both fluids test results show that the Ti $6 \mathrm{Al}-4 \mathrm{~V}$ material produced a larger capillary performance than the CP-2 titanium material. The CP-2 titanium wick pore size is much smaller than that of the Ti $6 \mathrm{Al}-4 \mathrm{~V}$ titanium wick. Thus there were much fewer flow channels for liquid penetrations in the CP-2 titanium wick, and the permeability was much lower than the Ti $6 \mathrm{Al}-4 \mathrm{~V}$ wick, as shown in table 1 . Though the pore size between adjacent $\mathrm{Ti} 6 \mathrm{Al}-4 \mathrm{~V}$ titanium particles were larger than those of $\mathrm{CP}-2$ titanium wicks, the capillary pressure of $\mathrm{Ti} 6 \mathrm{Al}-4 \mathrm{~V}$ titanium wick was found to be only slightly smaller than that of 
the CP-2 titanium wick as shown in table. 5.1. Therefore, a larger $\Delta P_{c} . K$ can be expected for the Ti 6Al-4V titanium wicks, which appears to be more advantageous for the application of porous wicks.

The condensation flow of wet steam was much quicker than that dry steam, as dry steam presents a much larger viscosity than the wet steam in spite that the surface tension and density of these two fluids are nearly the same. However, the discrepancies of $\Delta P_{c} . K$ for both titanium and nickel porous wicks between these two fluids tests were found to be very small. This indicates a good agreement between these two fluids tests to characterize the capillary performance. If using the normalized parameter $K / r_{\text {eff }}$ by eliminating the surface tension effect of different fluids, the results for the nickel porous wicks exhibited a fairly good agreement, as shown in table 5.1. The discrepancies for the titanium porous wicks were slightly larger, which may be due to the slight difference of contact angle formed between these two working fluids and the titanium wicks.

For the comparison of the current results with previous studies in the literatures, the comparison results of permeability and capillary performance parameters of titanium porous wicks showed good consistence with other studies by Holley and Faghri [69] and Lin et al [70]. The current four porous wicks exhibited a similar capillary behavior as the test results of nickel wick by Holley and Faghri [69], though the working fluid of methanol was employed in their study. Nevertheless, since a different data reduction method, i.e., closed form solution analysis, was utilized in their study, a larger permeability and effective pore radius were accessed. From the above evaluation, using the modeling approaches as described before, the energy, mass and momentum balances provide an accurate means to determine the capillary performance parameters of porous wicks. 
Table 5.1. Properties of different porous mediums and working fluids

\begin{tabular}{|c|c|c|c|c|c|c|}
\hline $\begin{array}{l}\text { Wick } \\
\text { material }\end{array}$ & $\begin{array}{l}\text { Particle size, } \\
\qquad(\mu \mathrm{m})\end{array}$ & $\begin{array}{l}\text { Specific } \\
\text { porosity } \\
\qquad(\varepsilon)\end{array}$ & Working Fluid & $\begin{array}{c}\text { Permeability } K \\
\left(m^{2}\right)\end{array}$ & $\begin{array}{c}\text { Effective } \\
\text { pore radius } \\
r_{e f f}(\mu \mathrm{m})\end{array}$ & $K / r_{e f f}(\mu m)$ \\
\hline Ti 6Al-4V & $50-90$ & 0.586 & $\begin{array}{l}\text { Dry steam } \\
\text { Wet steam }\end{array}$ & $7.81 \times 10^{-12}$ & $\begin{array}{l}11.2 \\
11.8\end{array}$ & 0.69 \\
\hline $\begin{array}{c}\text { CP-2 } \\
\text { Titanium }\end{array}$ & $50-90$ & 0.595 & $\begin{array}{l}\text { Dry steam } \\
\text { Wet steam }\end{array}$ & $5.22 \times 10^{-12}$ & $\begin{array}{c}9.9 \\
10.5\end{array}$ & 0.654 \\
\hline T123 Nickel & $3.0-8.0$ & 0.615 & $\begin{array}{l}\text { Dry steam } \\
\text { Wet steam }\end{array}$ & $0.34 \times 10^{-12}$ & $\begin{array}{l}1.6 \\
1.7\end{array}$ & $\begin{array}{l}0.217 \\
0.204\end{array}$ \\
\hline T200 Nickel & $2.25-2.75$ & 0.641 & $\begin{array}{l}\text { Dry steam } \\
\text { Wet steam }\end{array}$ & $0.88 \times 10^{-12}$ & $\begin{array}{l}2.7 \\
2.8\end{array}$ & $\begin{array}{l}0.328 \\
0.316\end{array}$ \\
\hline $\begin{array}{c}\text { Holley and } \\
\text { Faghri [69], } \\
\text { Nickel }\end{array}$ & - & 0.90 & Methanol & $3.40 \times 10^{-12}$ & 11.9 & 0.285 \\
\hline $\begin{array}{c}\text { Lin et al [70] } \\
\text { Nickel }\end{array}$ & $2.2-2.8$ & 0.74 & - & $2 \times 10^{-12}$ & 3 & 0.666 \\
\hline
\end{tabular}

\subsection{SUMMARY}

The capillary performance of porous wicks, which integrates both capillary pressure and permeability, determines the operation and performance of the porous wick. Four kinds of porous wicks, i.e., two type T200 and T123 nickel wicks with particle sizes of $2.25-2.75 \mu m$ and 3.0-8.0 $\mu \mathrm{m}$ respectively, and two $\mathrm{CP}-2$ and $\mathrm{Ti} 6 \mathrm{Al}-4 \mathrm{~V}$ titanium wicks of 50.0-90.0 $\mu \mathrm{m}$, were characterized for the purpose of wick design in the vapor condensation heat exchanger. Two working fluids, dry steam and wet steam from power plant were assessed. The results show that the capillary performance determined by both working fluids showed good agreement. The titanium porous wicks presented both larger permeability and capillary performance than the nickel wicks. The type T200 nickel porous wick outweighed the type T123 in both permeability and capillary performance. 


\section{CHAPTER SIX: CONCLUSIONS}

In order to increase the heat transfer rate of the vapor condensation heat exchanger, the current investigation focused on the condensation heat transfer and forced convection. To increase the heat transfer coefficient, the elliptical pin fins with nanofluid was investigated. The forced convective heat transfer on nanofluids in an elliptical pin-fin heat sink of two different pin orientations was studied by using a finite volume method. With increasing Reynolds number, the recirculation zones behind the pins increased. There were more recirculation zones for the pins with different angular orientations than for pins with the same angular orientation. It was observed that the Nusselt number for the pins with different angular orientations was higher than that for pins with the same angular orientation. The results showed that with increasing volume fraction of nanoparticles and angular orientation of pins for a given Reynolds number, Euler and Nusselt numbers as well as overall heat transfer efficiency increase. By utilizing thin film evaporation in the two-phase flow heat exchanger, investigations were performed for heat flux and pressure distributions. The maximum liquid pressure difference continuously increases with the superheat. The maximum liquid pressure difference peaks at about $5^{\circ} \mathrm{C}$ superheat. The curvature and interface temperature profiles were different at different superheats. A decrease in temperature occurred when the thin film profile increased in thickness. Both the $x$ and $y$ dimensionless coordinates and superheat at various thin film profiles were viewed and there were two crests in regions $\left(x^{*}>4.0, y^{*}<0.5\right)$ and $\left(x^{*}<2.0, y^{*}>1.5\right)$, and two shallow troughs in regions $\left(x^{*}<2.0, y^{*}<0.5\right)$ and $\left(x^{*}>4.0, y^{*}>1.5\right)$ where thin film profile was optimum. For the vapor condensation, in order to increase the condensation heat transfer rate, porous medium was utilized to increase the condensation area, and at the same time, the condensate could effectively be removed by the capillary force. The condensation-front speed and liquid-phase distribution in 
the condensate region were studied. Three different regions and a condensation zone were defined. The dimensionless vapor parameters of the problems were $J a, B o, p_{0}{ }^{*}$, and the thermo physical properties. The dimensionless front speed $u_{F}^{*}$ or the front location $x_{F}^{*}$ were found for the down flow of vapor in the porous medium. For vapor flow, a liquid region was present and increased in thickness with the distance from the inlet of vapor. The ratio of condensation front velocity to bond number $u_{F}^{*} / B o$ changed with respect to $x_{F}^{*} J a / p_{0}^{*}$ and $u_{V}^{*} / B o$ was a function of $x_{F}^{*} J a / p_{0}^{*}$. Four kinds of porous medium (wicks); two type T200 and T123 of nickel wicks with particle sizes of $2.25-2.75 \mu \mathrm{m}$ and $3.0-8.0 \mu \mathrm{m}$ respectively, and two $\mathrm{CP}-2$ and $\mathrm{Ti} 6 \mathrm{Al}-4 \mathrm{~V}$ titanium wicks with particle sizes of $50.0-90.0 \mu \mathrm{m}$, were characterized for the purpose of wick design in the vapor condensation heat exchanger. Two working fluids, dry steam and wet steam were studied. The results show that the capillary performance determined by both working fluids showed good agreement. The titanium porous wicks presented both larger permeability and capillary performance than the nickel wicks. The type T200 nickel porous wick outweighed the type T123 in both permeability and capillary performance. 


\section{APPENDICES}

\section{APPENDIX A: POROUS MEDIUM MATHEMATICAL DERIVATIONS}

The total pressure loss as sum of the pressure losses for vapor, liquid, capillary and air from Eq. (197),

$$
\Delta p=p_{v}(0)-p_{v}\left(\mathrm{Z}_{v}\right)+\Delta p_{l}+\Delta p_{i}+\Delta p_{c}
$$

where

$$
\begin{gathered}
p_{v}(z)=\sqrt{p_{v}(0)^{2}-\frac{z}{Z_{v}}\left(p_{v}(0)^{2}-p_{v}(z)^{2}\right)} \\
p_{v}\left(\mathrm{z}=Z_{v}\right)=\sqrt{p_{v}(0)^{2}-\left(p_{v}(0)^{2}-p_{v}\left(Z_{v}\right)^{2}\right)}
\end{gathered}
$$

from Eq. (A.3), and by taking square root of $p_{v}(0)$, Eq. (A.1) can be expressed as

$$
\Delta p=p_{v}(0)-p_{v}(0) \sqrt{1-\frac{1}{p_{v}(0)^{2}}\left(p_{v}(0)^{2}-p_{v}\left(\mathrm{Z}_{v}\right)^{2}\right)}+\Delta p_{l}+\Delta p_{i}+\Delta p_{c}
$$

by knowing the following expression from Eq. (182),

$$
p_{v}(0)^{2}-p_{v}\left(\mathrm{Z}_{v}\right)^{2}=2 p_{0} Z_{v}\left(\frac{\bar{\mu}_{v, S}}{K_{v}} u_{v, D, 0}+\frac{\rho_{v, 0}}{K_{v}^{*}} u_{v, D, 0}^{2}\right)
$$

and by knowing the following two expressions

$$
\Delta p_{l}=\frac{\mu_{l}}{K_{l}} \varepsilon u_{l} \Delta Z_{l}, \Delta p_{i}=\frac{\mu_{i}}{K_{i}} \varepsilon u_{i} \Delta Z_{i}
$$

and that $p_{v}(0)=p_{0}$, Eq. (A.4) can be written as

$$
\Delta p=p_{v}(0)-p_{v}(0) \sqrt{1-2 \frac{Z_{v}}{p_{0}}\left(\frac{\bar{\mu}_{v, S}}{K_{v}} u_{v, D, 0}+\frac{\rho_{v, 0}}{K_{v}^{*}} u_{v, D, 0}^{2}\right)}+\frac{\mu_{l}}{K_{l}} \varepsilon u_{l} \Delta Z_{l}+\frac{\mu_{i}}{K_{i}} \varepsilon u_{i} \Delta Z_{i}+\frac{2 \sigma}{r_{\text {eff }}}+\frac{2 \gamma}{R\left(\frac{8}{\pi}-1\right)}
$$




$$
\Delta p=p_{v}(0)-p_{v}(0) \sqrt{1-2 \frac{Z_{v}}{p_{0}} u_{v, D, 0}\left(\frac{\bar{\mu}_{v, S}}{K_{v}}+\frac{\rho_{v, 0}}{K_{v}^{*}} u_{v, D, 0}\right)}+\frac{\mu_{l}}{K_{l}} \varepsilon u_{l} \Delta Z_{l}+\frac{\mu_{i}}{K_{i}} \varepsilon u_{i} \Delta Z_{i}+\frac{2 \sigma}{r_{\text {eff }}}+\frac{2 \gamma}{R\left(\frac{8}{\pi}-1\right)}(\mathrm{A} .8)
$$

now from Eq. (180),

$$
u_{v, D, 0}=\varepsilon_{v} u_{v, 0}
$$

Eq. (A.8) becomes

$$
\Delta p=p_{v}(0)-p_{v}(0) \sqrt{1-2 \frac{\varepsilon_{v} u_{v, 0}}{p_{0}}\left(\frac{\bar{\mu}_{v, S}}{K_{v}}+\frac{\rho_{v, 0}}{K_{v}^{*}} \varepsilon_{v} u_{v, 0}\right) Z_{v}}+\frac{\mu_{l}}{K_{l}} \varepsilon u_{l} \Delta Z_{l}+\frac{\mu_{i}}{K_{i}} \varepsilon u_{i} \Delta Z_{i}+\frac{2 \sigma}{r_{\text {eff }}}+\frac{2 \gamma}{R\left(\frac{8}{\pi}-1\right)}(\mathrm{A}
$$

for times before liquid become stagnant, $Z_{v}+\Delta Z_{l} \leq L$, by knowing the following expressions,

$$
\begin{gathered}
\Delta Z_{i}=L-Z_{v}-\Delta Z_{l} \\
\Delta Z_{l}=\zeta Z_{l} \\
\Delta Z_{i}=L-Z_{v}(1+\zeta) \\
u_{l}=u_{i}=\frac{d Z_{v}}{d t}(1+\zeta) \\
u_{v, 0}=\frac{d Z_{v}}{d t}\left(\frac{\xi}{\varepsilon_{v} \rho_{v, 0}}\right)
\end{gathered}
$$

the second term in the square root of Eq. (A.10) can be written as

$$
2 \frac{\varepsilon_{v}}{p_{0}} \frac{d Z_{v}}{d t}\left(\frac{\xi}{\varepsilon_{v} \rho_{v, 0}}\right)\left(\frac{\bar{\mu}_{v, S}}{K_{v}}+\frac{\rho_{v, 0}}{K_{v}^{*}} \varepsilon_{v} \frac{d Z_{v}}{d t}\left(\frac{\xi}{\varepsilon_{v} \rho_{v, 0}}\right)\right) Z_{v}=\frac{2}{p_{0}} \frac{d Z_{v}}{d t}\left(\frac{\xi}{\rho_{v, 0}}\right)\left(\frac{\bar{\mu}_{v, S}}{K_{v}}+\frac{d Z_{v}}{d t}\left(\frac{\xi}{K_{v}^{*}}\right)\right) Z_{v}(\mathrm{~A}
$$

rearranging yields,

$$
2 \frac{\varepsilon_{v}}{p_{0}} \frac{d Z_{v}}{d t}\left(\frac{\xi}{\varepsilon_{v} \rho_{v, 0}}\right)\left(\frac{\bar{\mu}_{v, S}}{K_{v}}+\frac{\rho_{v, 0}}{K_{v}^{*}} \varepsilon_{v} \frac{d Z_{v}}{d t}\left(\frac{\xi}{\varepsilon_{v} \rho_{v, 0}}\right)\right) Z_{v}=2 \frac{\xi^{2}}{\rho_{v, 0} p_{0} K_{v}^{*}}\left(\frac{\bar{\mu}_{v, S} K_{v}^{*}}{\xi K_{v}}+\frac{d Z_{v}}{d t}\right) \frac{d Z_{v}}{d t} Z_{v}(\mathrm{~A}
$$

so the final form Eq. (A.10) can be expressed as follows 


$$
\begin{aligned}
& \Rightarrow \Delta p=p_{v}(0)-p_{v}(0) \sqrt{1-\frac{2 \xi^{2}}{\rho_{v, 0} p_{0} K_{v}^{*}}\left(\frac{\bar{\mu}_{v, S} K_{v}^{*}}{\xi K_{v}}+\frac{d Z_{v}}{d t}\right) \frac{d Z_{v}}{d t} Z_{v}} \\
&+\left[\frac{\mu_{l}}{K_{l}} \varepsilon \zeta Z_{v}+\frac{\mu_{i}}{K_{i}} \varepsilon Z_{v}(\zeta+1)\right](1+\zeta) \frac{d Z_{v}}{d t}+\frac{2 \sigma}{r_{e f f}}+\frac{2 \gamma}{R\left(\frac{8}{\pi}-1\right)}
\end{aligned}
$$

after the condensate region has reached the exit, and by using

$$
\Delta Z_{i}=0 \& \Delta Z_{l}=L-Z_{v}
$$

Eq. (A.18) can be written as

$$
\Delta p=p_{v}(0)-p_{v}(0) \sqrt{1-\frac{2 \xi^{2}}{\rho_{v, 0} p_{0} K_{v}^{*}}\left(\frac{\bar{\mu}_{v, S} K_{v}^{*}}{\xi K_{v}}+\frac{d Z_{v}}{d t}\right) \frac{d Z_{v}}{d t} Z_{v}}+\left[\frac{\mu_{l}}{K_{l}} \varepsilon(\zeta+1)\left(L-Z_{v}\right)\right] \frac{d Z_{v}}{d t}+\frac{2 \sigma}{r_{\text {eff }}}+\frac{2 \gamma}{R\left(\frac{8}{\pi}-1\right)}(A
$$

the general form of Eqs. (209) and (210) i.e.,

$$
\sqrt{1-C_{1} Z_{v}\left(C_{2}+\frac{d Z_{v}}{d t}\right) \frac{d Z_{v}}{d t}}=\left(C_{3} Z_{v}+C_{4}\right) \frac{d Z_{v}}{d t}+C_{5}
$$

with the constants

$$
\begin{gathered}
C_{1}=\frac{2 \xi^{2}}{\rho_{v, 0} p_{0} K_{0}^{*}} \\
C_{2}=\frac{\overline{\mu_{v, S}} K_{v}^{*}}{\xi K_{v}} \\
C_{5}=\frac{p_{0}-\Delta p}{p_{0}} \\
C_{3}=\frac{\zeta+1}{p_{0}}\left[\frac{\mu_{l}}{K_{v}} \varepsilon \zeta-\frac{\mu_{i}}{K_{i}} \varepsilon(\zeta+1)\right]
\end{gathered}
$$

Before the condensate reaches the exit, $Z_{v}+\Delta Z_{l} \leq L$, the process is described by Eq. (209) and the constant is 


$$
C_{4}=\frac{\zeta+1}{p_{0}} \frac{\mu_{i}}{K_{i}} \varepsilon L
$$

After liquid become stagnant, $Z_{v}+\Delta Z_{l} \geq L$ and the constants are

$$
\begin{gathered}
C_{3}=\frac{\zeta+1}{p_{0}} \frac{\mu_{l}}{K_{l}} \varepsilon \\
C_{4}=\frac{\zeta+1}{p_{0}} \frac{\mu_{l}}{K_{l}} \varepsilon L
\end{gathered}
$$

so the solutions of Eq. (A.21),

$$
\begin{gathered}
{\left[\sqrt{1-C_{1} Z_{v}\left(C_{2}+\frac{d Z_{v}}{d t}\right) \frac{d Z_{v}}{d t}}\right]^{2}=\left[\left(C_{3} Z_{v}+C_{4}\right) \frac{d Z_{v}}{d t}+C_{5}\right]^{2}} \\
1-C_{1} Z_{v}\left(C_{2}+\frac{d Z_{v}}{d t}\right) \frac{d Z_{v}}{d t}=\left(C_{3} Z_{v}+C_{4}\right)^{2}\left(\frac{d Z_{v}}{d t}\right)^{2}+2\left(C_{3} Z_{v}+C_{4}\right) C_{5} \frac{d Z_{v}}{d t}+C_{5}^{2} \\
f_{1}\left(Z_{v}\right)=\left(C_{3} Z_{v}+C_{4}\right)^{2}+C_{1} Z_{v} \\
f_{2}\left(Z_{v}\right)=2\left(C_{3} Z_{v}+C_{4}\right) C_{5}+C_{1} C_{2} Z_{v} \\
C_{6}=1-C_{5}
\end{gathered}
$$

where

$$
\begin{gathered}
f_{1}\left(\mathrm{Z}_{v}\right)\left(\frac{d Z_{v}}{d t}\right)^{2}+f_{2}\left(\mathrm{Z}_{v}\right) \frac{d Z_{v}}{d t}=C_{6} \\
\frac{f_{1}\left(\mathrm{Z}_{v}\right)}{\left(\frac{d t}{d Z_{v}}\right)^{2}}+\frac{f_{2}\left(\mathrm{Z}_{v}\right)}{\frac{d t}{d Z_{v}}}=C_{6}
\end{gathered}
$$

using Eqs. (A.31) and (A.32), the solution of Eq. (A.35) can be written as 


$$
\frac{d t}{d Z_{v}}=\frac{f_{2}\left(\mathrm{Z}_{v}\right) \pm \sqrt{f_{2}\left(\mathrm{Z}_{v}\right)^{2}+4 f_{2}\left(\mathrm{Z}_{v}\right) C_{6}}}{2 C_{6}}
$$

rearranging yields,

$$
\frac{f_{2}\left(\mathrm{Z}_{v}\right) \pm \sqrt{f_{2}\left(\mathrm{Z}_{v}\right)^{2}+4 f_{2}\left(\mathrm{Z}_{v}\right) C_{6}}}{2 C_{6}} \frac{d Z_{v}}{d t}=1
$$

only a solution with the positive sign leads to a physically meaningful result, therefore Eq. (A.37) is to be solved by integration i.e.,

$$
\frac{1}{2 C_{6}}\left(\int f_{2}\left(\mathrm{Z}_{v}\right) d Z_{v}+\int \sqrt{f_{2}\left(\mathrm{Z}_{v}\right)^{2}+4 f_{2}\left(\mathrm{Z}_{v}\right) C_{6}} d \mathrm{Z}_{v}\right)=\int d t
$$

rearranging of Eq. (A.32), first left term of Eq. (A.38) yields,

$$
f_{2}\left(Z_{v}\right)=2\left(C_{3} Z_{v}+C_{4}\right) C_{5}+C_{1} C_{2} Z_{v}=\left(C_{3} C_{5}+C_{1} C_{2}\right) Z_{v}+2 C_{4} C_{5}=\left(C_{7} Z_{v}+C_{8}\right)
$$

where,

$$
\begin{gathered}
C_{7}=C_{3} C_{5}+C_{1} C_{2} \\
C_{8}=2 C_{4} C_{5}
\end{gathered}
$$

substituting Eq. (A.39) into Eq. (A.38), second left term of Eq. (A.38) yields,

$$
\begin{aligned}
& \sqrt{f_{2}\left(\mathrm{Z}_{v}\right)^{2}+4 f_{2}\left(\mathrm{Z}_{v}\right) C_{6}}=\sqrt{\left(C_{7} Z_{v}+C_{8}\right)^{2}+4 C_{6}\left(\left(C_{3} Z_{v}+C_{4}\right)^{2}+C_{1} Z_{v}\right.}= \\
& \sqrt{C_{7}^{2} Z_{v}^{2}+2 C_{7} C_{8} Z_{v}+C_{8}^{2}+4 C_{6}\left(C_{3}^{2} X_{v}^{2}+2 C_{3} C_{4} Z_{v}+C_{4}^{2}+C_{1} Z_{v}\right)}=\sqrt{C_{9} Z_{v}^{2}+C_{10} Z_{v}+C_{11}}
\end{aligned}
$$

where,

$$
\begin{gathered}
C_{9}=C_{4}^{2}+4 C_{3} C_{6} \\
C_{10}=2 C_{7} C_{8}+4\left(C_{1}+2 C_{3} C_{4}\right) C_{6} \\
C_{11}=C_{8}^{2}+4 C_{4} C_{6}
\end{gathered}
$$

now Eq. (A.38) can be rewritten as follows 


$$
\frac{1}{2 C_{6}}\left(\int\left(C_{7} Z_{v}+Z_{8}\right) d Z_{v}+\int \sqrt{C_{9} Z_{v}^{2}+C_{10} Z_{v}+C_{11}} d Z_{v}\right)=\int d t
$$

and by integrating Eq. (A.46), i.e.,

$>t:=\operatorname{int}(C[7] * Z[v]+C[8], Z[v])+\operatorname{int}\left(\operatorname{sqrt}\left(C[9]^{*} Z[v]^{\wedge} 2+C[10]^{*} Z[v]+C[11]\right), Z[v]\right) ;$

$$
\begin{array}{r}
\frac{1}{2} Z_{v}^{2} C_{7}+C_{8} Z_{v}+\frac{1}{4} \frac{\left(2 C_{9} Z_{v}+C_{10}\right) \sqrt{C_{9} Z_{v}^{2}+C_{10} Z_{v}+C_{11}}}{C_{9}} \\
\left.+\frac{1}{2} \frac{\ln \left(\frac{\frac{1}{2} C_{10}+C_{9} Z_{v}}{\sqrt{C_{9}}}+\sqrt{C_{9} Z_{v}^{2}+C_{10} Z_{v}+C_{11}}\right) C_{11}}{\sqrt{C_{9}}}\right) C_{10}^{2} \\
-\frac{1}{8} \frac{\ln \left(\frac{\frac{1}{2} C_{10}+C_{9} Z_{v}}{\sqrt{C_{9}}}+\sqrt{C_{9} Z_{v}^{2}+C_{10} Z_{v}+C_{11}}\right.}{C_{9}^{(3 / 2)}}
\end{array}
$$

and by multiplying by $\left(1 / 2 C_{6}\right)$, and also by adding the capillary terms and rearranging, the final result is as follows,

$$
\begin{gathered}
t=\frac{1}{2} \frac{C_{7} Z_{v}^{2}}{2 C_{6}}+\frac{C_{8} Z_{v}}{2 C_{6}}+\frac{1}{4} \frac{\left(C_{9} Z_{v}+\frac{C_{10}}{2}\right) \sqrt{C_{9} Z_{v}^{2}+C_{10} Z_{v}+C_{11}}}{C_{6} C_{9}}+\frac{1}{4} \frac{\ln \left(\frac{\left(\frac{1}{2} C_{10}+C_{9} Z_{v}\right)}{\sqrt{C_{9}}}+\sqrt{C_{9} Z_{v}^{2}+C_{10} Z_{v}+C_{11}}\right) C_{11}}{C_{6} \sqrt{C_{9}}} \\
+\frac{1}{16} \frac{\ln \left(\frac{\left(\frac{1}{2} C_{10}+C_{9} Z_{v}\right)}{\sqrt{C_{9}}}+\sqrt{C_{9} Z_{v}^{2}+C_{10} Z_{v}+C_{11}}\right) C_{10}^{2}}{C_{6} C_{9}^{(3 / 2)}}+\frac{1}{C_{6}} \frac{\sigma}{r_{\text {eff }}} \frac{\gamma}{R\left(\frac{8}{\pi}-1\right)}
\end{gathered}
$$



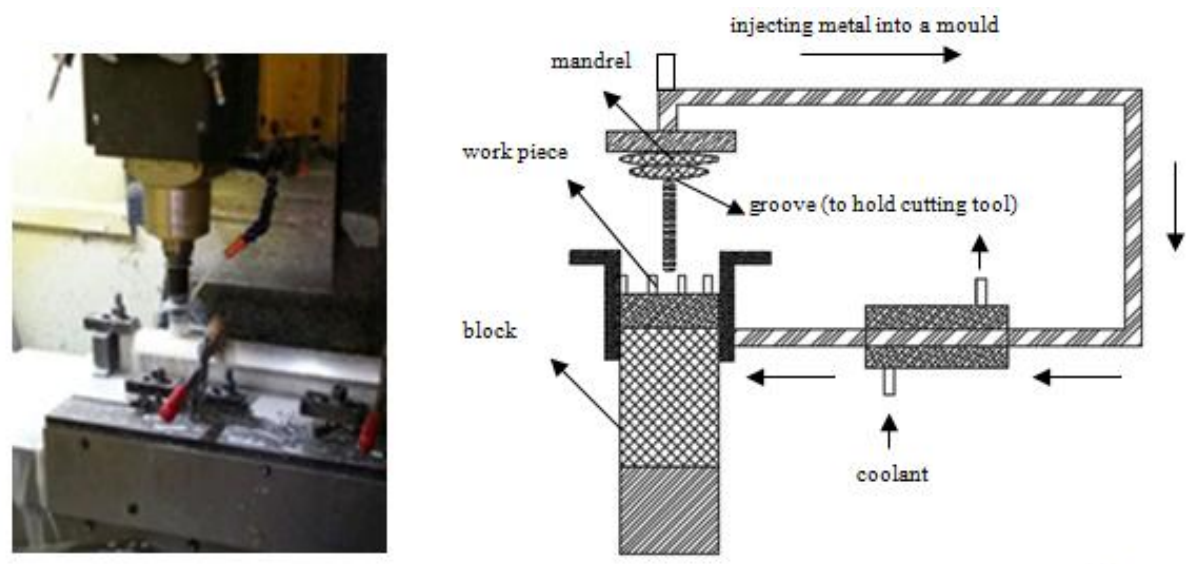

Figure B.1. Schematic of CNC machining

$\mathrm{CNC}$ metal injection molding was used in prototyping (II) of the three-dimensional pin fin heat sinks. A schematic of CNC machining is shown in figure B.1 and a photograph of experimental prototype is shown in figure B.2. A computer's unique software and control console set the proposed system for use in $\mathrm{CNC}$ machining. Under $\mathrm{CNC}$ machining, machine tools (grinding spindle, vice, cutter, lathe bed, cutter head) could function through a computer control. A computer program was customized for both pin fins and the metal injection molding machines were programmed with $\mathrm{CNC}$ machining language that essentially controlled all features like feed

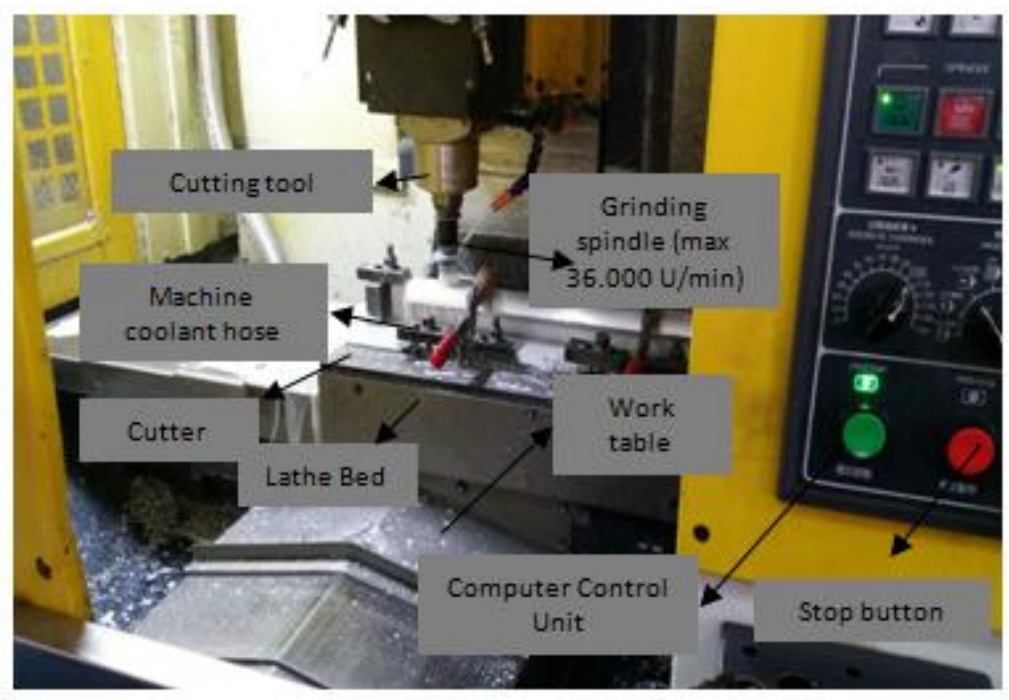


Figure B.2. Photograph of experimental prototype

rate, coordination, location and speeds. With $\mathrm{CNC}$ machining, the computer could control exact positioning and velocity. CNC machining was used in manufacturing of both pin fin heat sinks.

First 3D CAD drawings were created, and then a code was created that the CNC machine could understand. The program was loaded and the program was tested to ensure there were no problems. This trial was important step because any mistake with speed and tool position could result in a scraped part or a broken sword that could damage the machine. There were many advantages to using $\mathrm{CNC}$ machining process. This process was more precise than manual machining. Because of the precision possible with this process, it could produce current complex design that would be almost impossible to achieve with manual machining. Current CNC machining was used in a high level of precision, thus both three-dimensional pin fin shapes, the micro fins with same angular orientation and the micro fins with different angular orientation, were successfully produced (Figs. C.1-C.3, C.5).

\section{APPENDIX C: PROGRAMMING CODES}

\section{C1: HEAT SINK PROGRAMMING CODES}

$>\mathrm{k} \_\mathrm{Cu}:=400 ; \# \mathrm{~W} / \mathrm{m}-\mathrm{K} \quad$ k_Cu $:=400$

$>\mathrm{L}:=7.2 *(1 \mathrm{e}-3) ; \# \mathrm{~mm} \rightarrow \mathrm{m} \quad L:=0.0072$

$>\mathrm{d}:=3 *(1 \mathrm{e}-3) ; \quad d:=0.003$

$>\mathrm{k}:=0.620$; \#W/m_K $k:=0.620$

$>$ nu:=7.79e-7; \# m^2/s $v:=7.7910^{-7}$

$>\operatorname{Pr}:=5.2 ; \operatorname{Pr}:=5.2$

$>\mathrm{V}:=0.5 ; \# \mathrm{~m} / \mathrm{s} \quad V:=0.5$

$>$ theta $[\mathrm{b}]:=50 . ; \theta_{b}:=50$.

$>\operatorname{ReD}:=\mathrm{V} * \mathrm{~d} / \mathrm{nu} ; \operatorname{ReD}:=1925.545571$ 
$>\mathrm{NuD}:=0.3+0.62 * \operatorname{ReD}^{\wedge}(1 . / 2 .)^{*} \operatorname{Pr}^{\wedge}(1 . / 3 .)^{*}\left(1+(\operatorname{ReD} / 282000)^{\wedge}(5 . / 8).\right) /\left(1+(0.4 / \operatorname{Pr})^{\wedge}(2 . / 3 .)\right)^{\wedge}(1 . / 4$.$) ;$

$N u D:=47.51853511$

$>\mathrm{h}:=\mathrm{NuD} * \mathrm{k} / \mathrm{d} ; \quad h:=9820.497257$

$>\mathrm{P}:=\operatorname{evalf}(\mathrm{Pi}) * \mathrm{~d} ; \quad P:=0.009424777962$

$>\mathrm{Ac}:=\operatorname{evalf}(\mathrm{Pi}) * \mathrm{~d}^{\wedge} 2 / 4 ; \quad A c:=0.000007068583472$

$>\mathrm{M}:=\operatorname{theta}[\mathrm{b}] *\left(\mathrm{~h} * \mathrm{P} * \mathrm{k} \_\mathrm{Cu} * \mathrm{Ac}\right)^{\wedge}(1 / 2) ; \quad M:=25.57811282$

$>\mathrm{m}:=\left(\mathrm{h} * \mathrm{P} /\left(\mathrm{k} \_\mathrm{Cu} * \mathrm{Ac}\right)\right)^{\wedge}(1 / 2) ; m:=180.9281373$

$>\mathrm{mL}:=\mathrm{m}^{*} \mathrm{~L} ; \quad m L:=1.302682589$

$>\mathrm{h} /\left(\mathrm{m} * \mathrm{k} \_\mathrm{Cu}\right) ; 0.1356961029$

> $\mathrm{qf}:=\mathrm{M} *\left(\sinh (\mathrm{m} * \mathrm{~L})+\left(\mathrm{h} /\left(\mathrm{m} * \mathrm{k} \_\mathrm{Cu}\right)\right)^{*} \cosh \left(\mathrm{m}^{*} \mathrm{~L}\right)\right) /\left(\cosh (\mathrm{m} * \mathrm{~L})+\left(\mathrm{h} /\left(\mathrm{m}^{*} \mathrm{k} \_\mathrm{Cu}\right)\right) * \sinh (\mathrm{m} * \mathrm{~L})\right.$

$q f:=22.85508689$

$>\mathrm{q}:=\mathrm{h} *\left(\mathrm{w}^{\wedge} 2-\mathrm{N} * \mathrm{Ac}\right) * \operatorname{theta}[\mathrm{b}]+\mathrm{N} * \mathrm{qf} ; \quad q:=869.5694090$

$>\mathrm{As}:=\mathrm{W}^{\wedge} 2+\mathrm{N}^{*} \mathrm{P} * \mathrm{~L} ; \quad$ As $:=0.00248046003$

$>$ Ts: $=273+70 ;$ Ts $:=343$.

$>\mathrm{Ti}:=273+20 ; \quad T i:=293$.

$>$ Te:=273+35.; Te $:=308$.

$>$ dT_lm:=((Ts-Te)-(Ts-Ti))/ln((Ts-Te)/(Ts-Ti)); dT_lm $:=42.0550987$ !

> q:=h*As*dT_lm; $q:=1024.43491$

$>$

\section{C2: POROUS MEDIUM PROGRAMMING CODES}
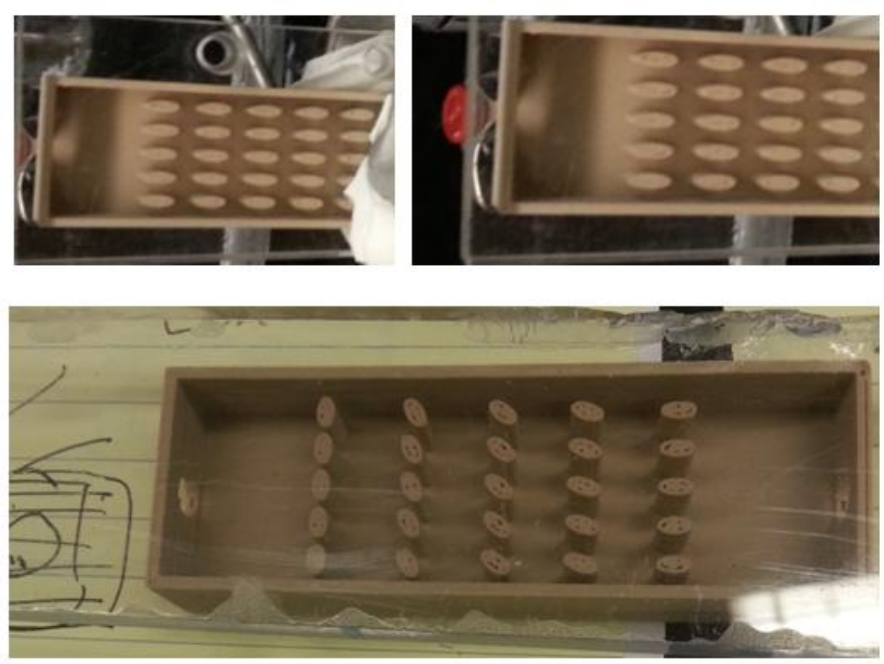

Figure C.1. Photograph of prototype I 


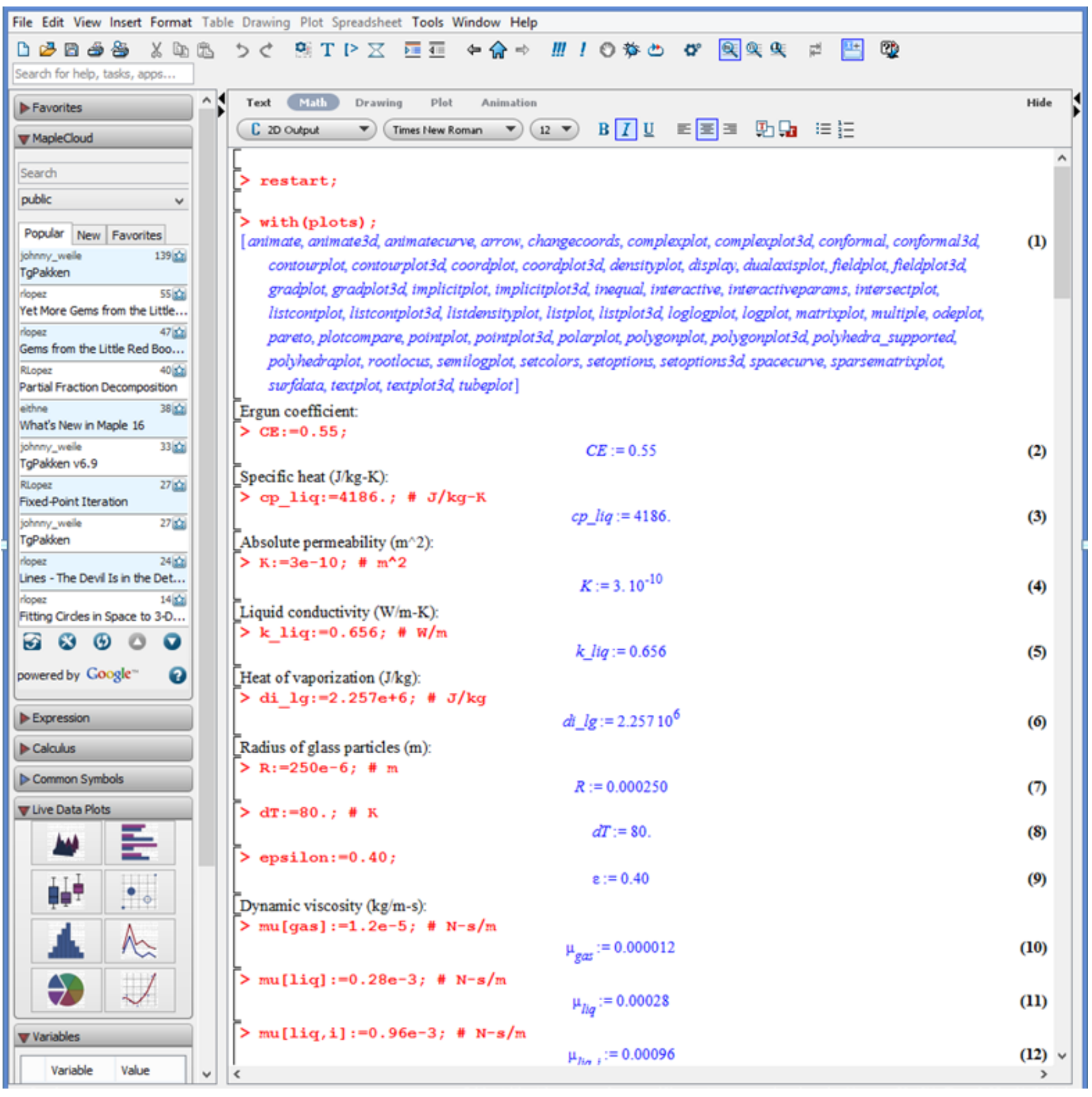




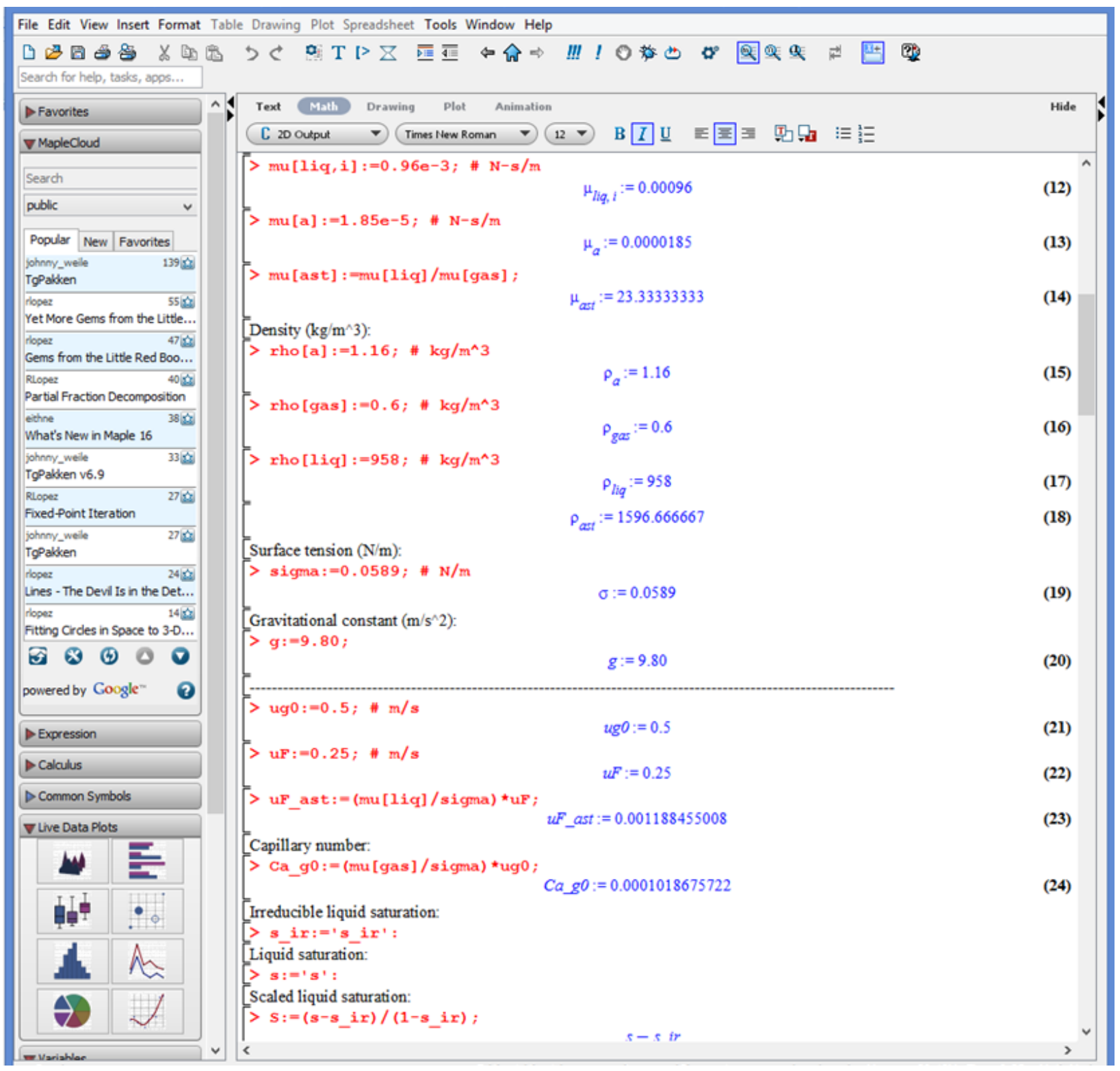



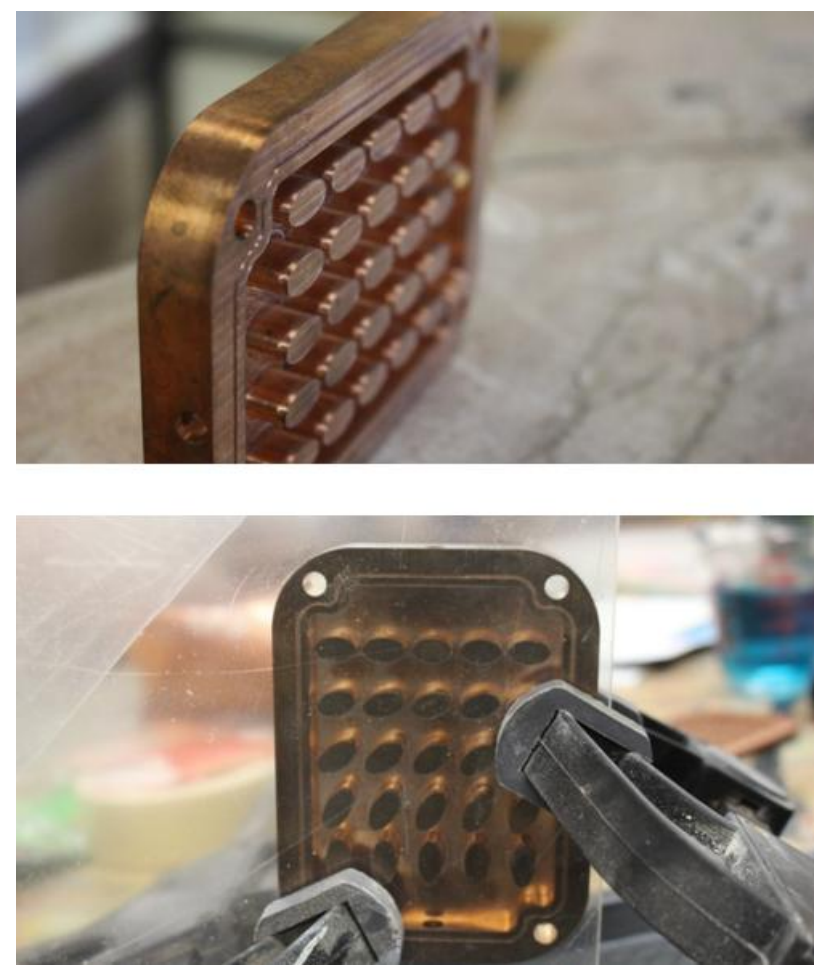

Figure C.2. Photograph of cnc machine prototype

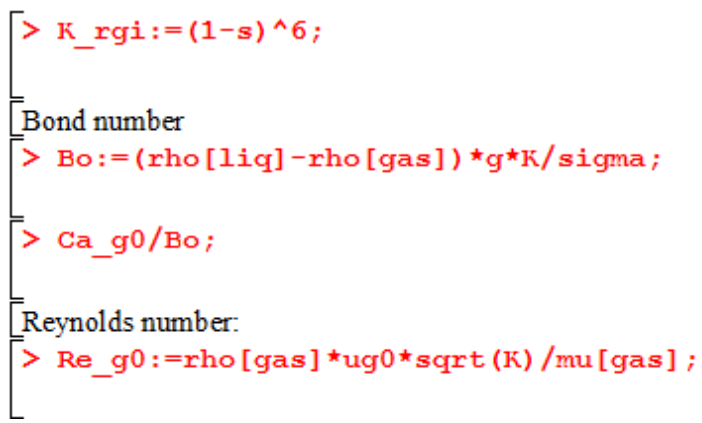

$$
\begin{gathered}
K_{-} r g i:=(1-s)^{6} \\
B o:=0.00004778872666 \\
2.131623488
\end{gathered}
$$$$
\text { Re_g0 }:=0.4330127020
$$
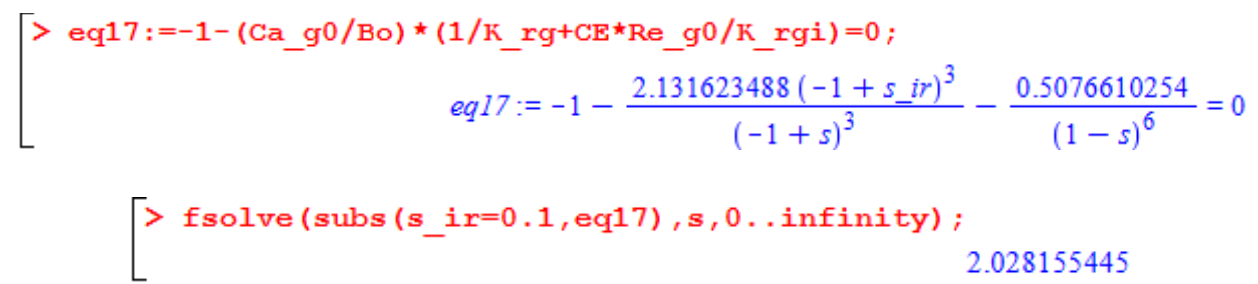

$$
\begin{aligned}
& {\left[\begin{array}{l}
\text { fsolve (subs (s_ir }=0.2, \text { eq } 17), s, 0 \ldots \text { infinity) } ; \\
\text { fsolve }\left(-1+\frac{1.091391226}{(-1+s)^{3}}-\frac{0.5076610254}{(1-s)^{6}}=0, s, 0 \ldots \infty\right)
\end{array}\right.}
\end{aligned}
$$

$\left[>\operatorname{plot}\left(\operatorname{subs}\left(s \_i r=0.10,-1-\left(\mathrm{Ca} \_g 0 / \mathrm{Bo}\right) *\left(1 / \mathrm{K} \_r g+\mathrm{CE}{ }^{\star} \operatorname{Re} \_g 0 / \mathrm{K} \_r g i\right)\right), s=0 \ldots 2,-100 \ldots 100\right)\right.$; 
$\left[>\operatorname{plot}\left(\operatorname{subs}\left(s \_i r=0.20,-1-\left(\mathrm{Ca} \_g 0 / \mathrm{Bo}\right){ }^{*}\left(1 / \mathrm{K} \_r g+\mathrm{CE}{ }^{\star} \mathrm{Re} \_g 0 / \mathrm{K} \_r g i\right)\right), s=0.2,-100 \ldots 100\right) ;\right.$

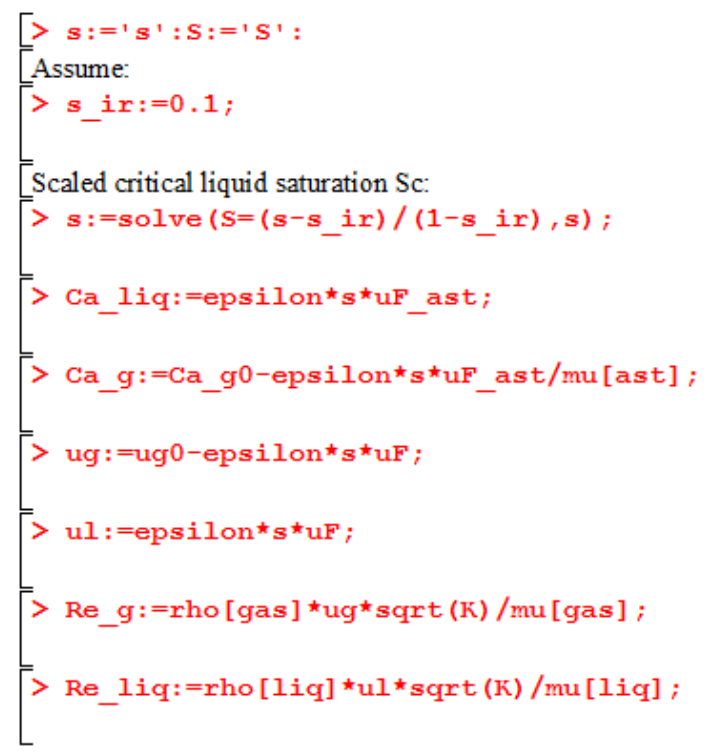

Scaled critical liquid saturation Sc:

$$
s_{-} i r:=0.1
$$

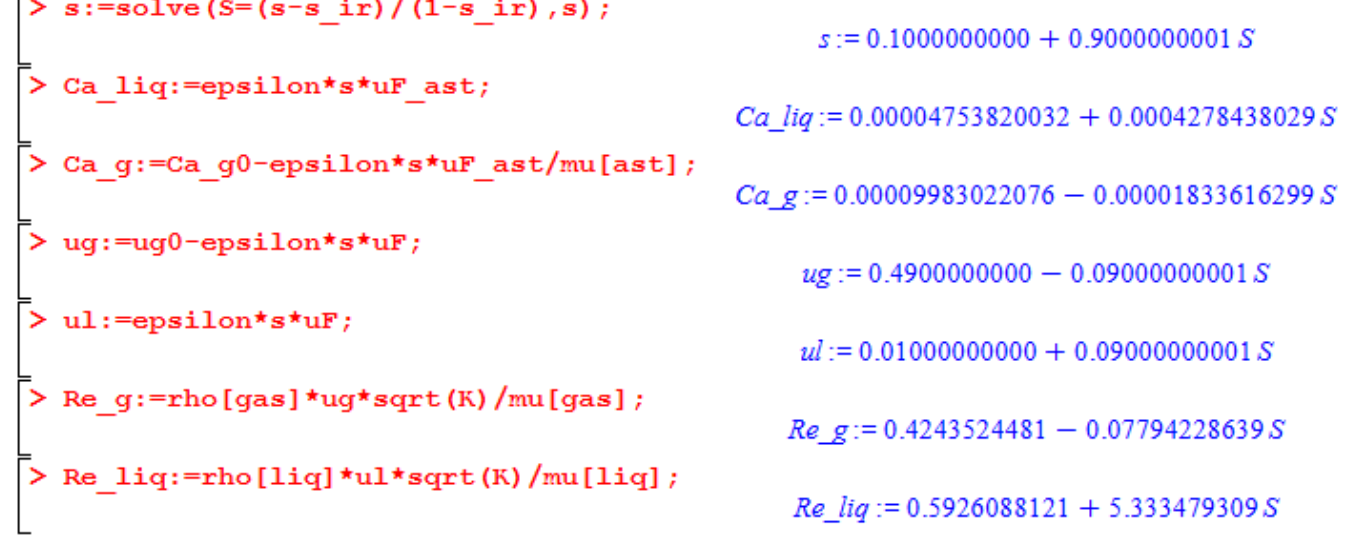

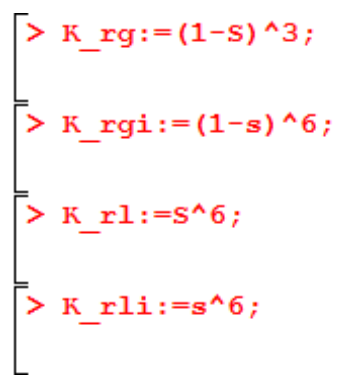

$K \_r g:=(1-S)^{3}$

K_rgi $:=(0.9000000000-0.9000000001 S)^{6}$

$$
K \_r l:=S^{6}
$$

$K \_r l i:=(0.1000000000+0.9000000001 S)^{6}$

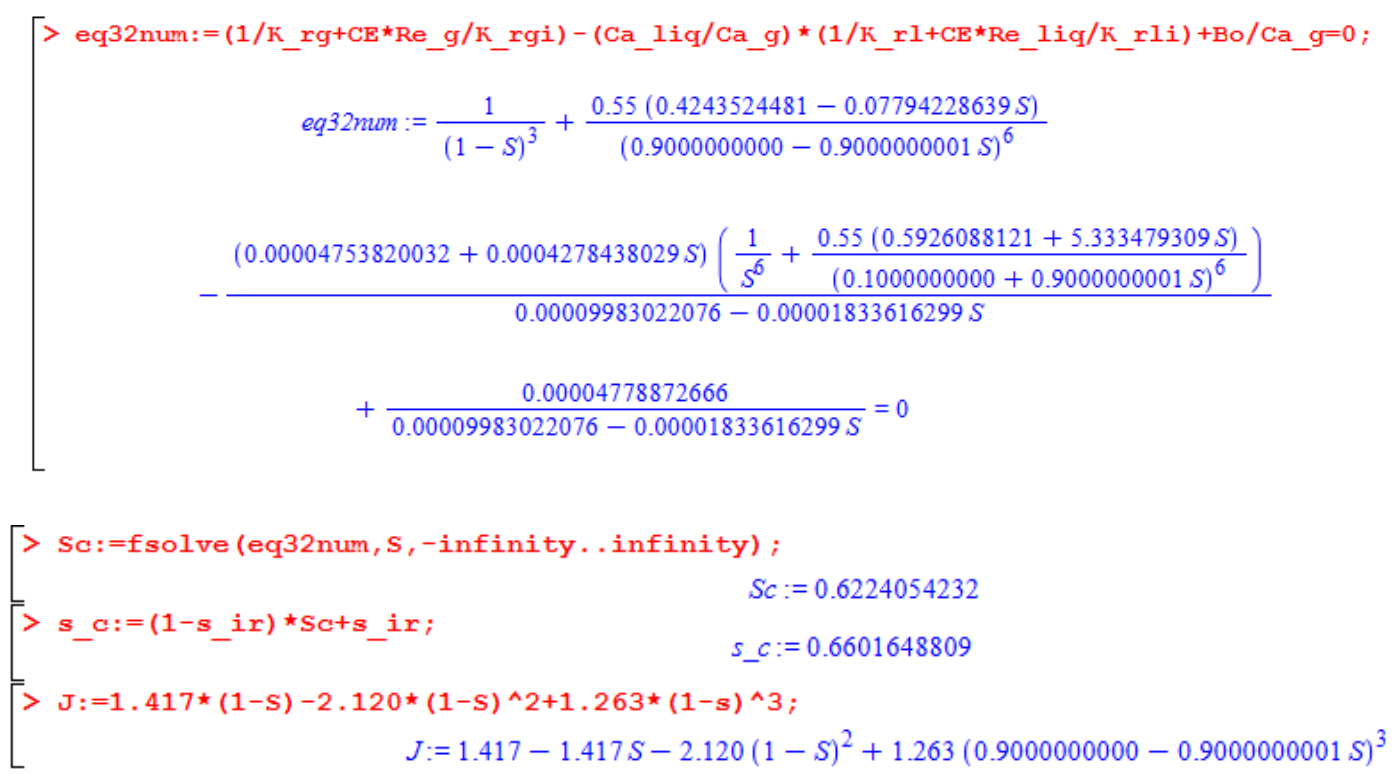




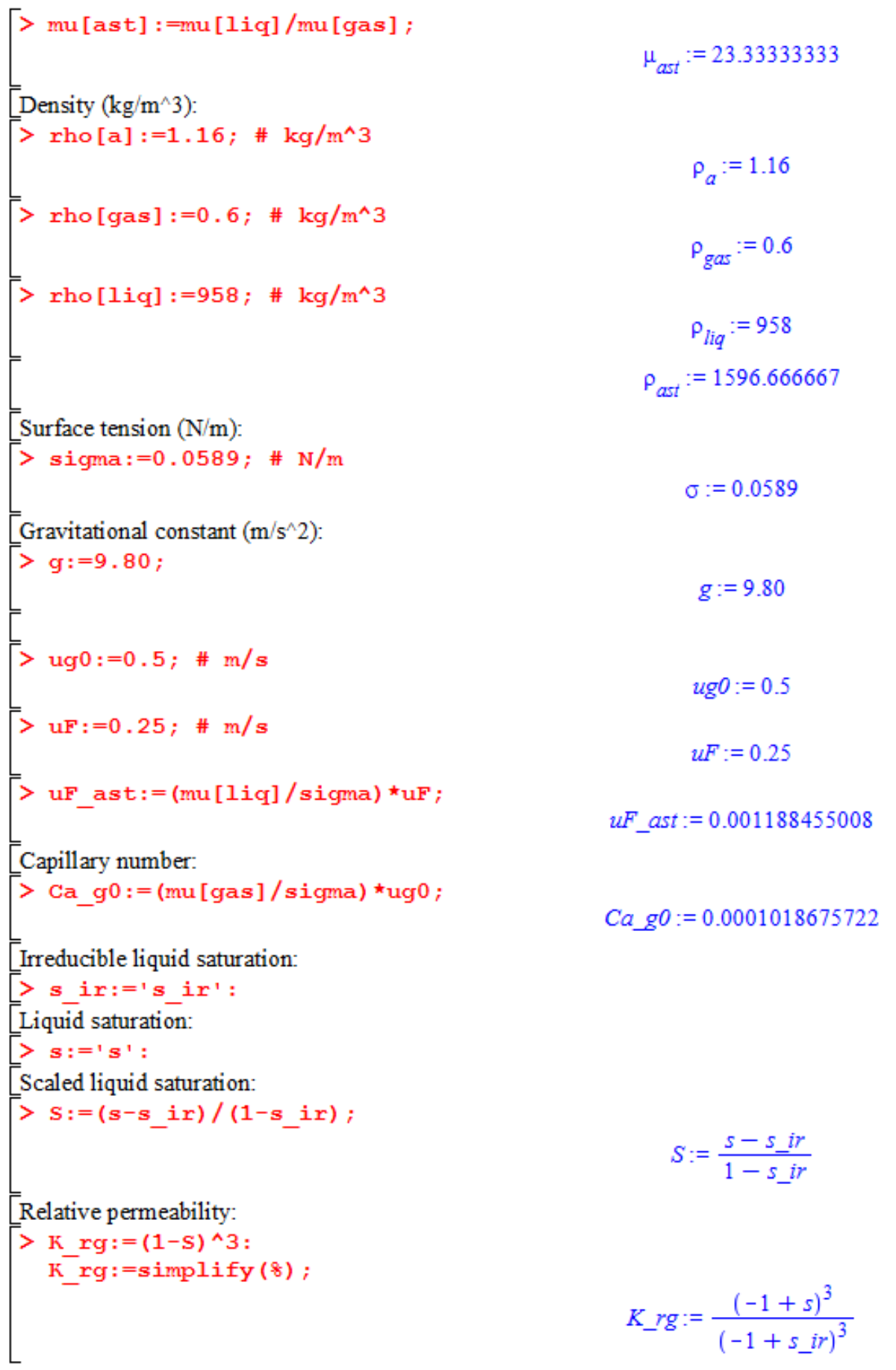




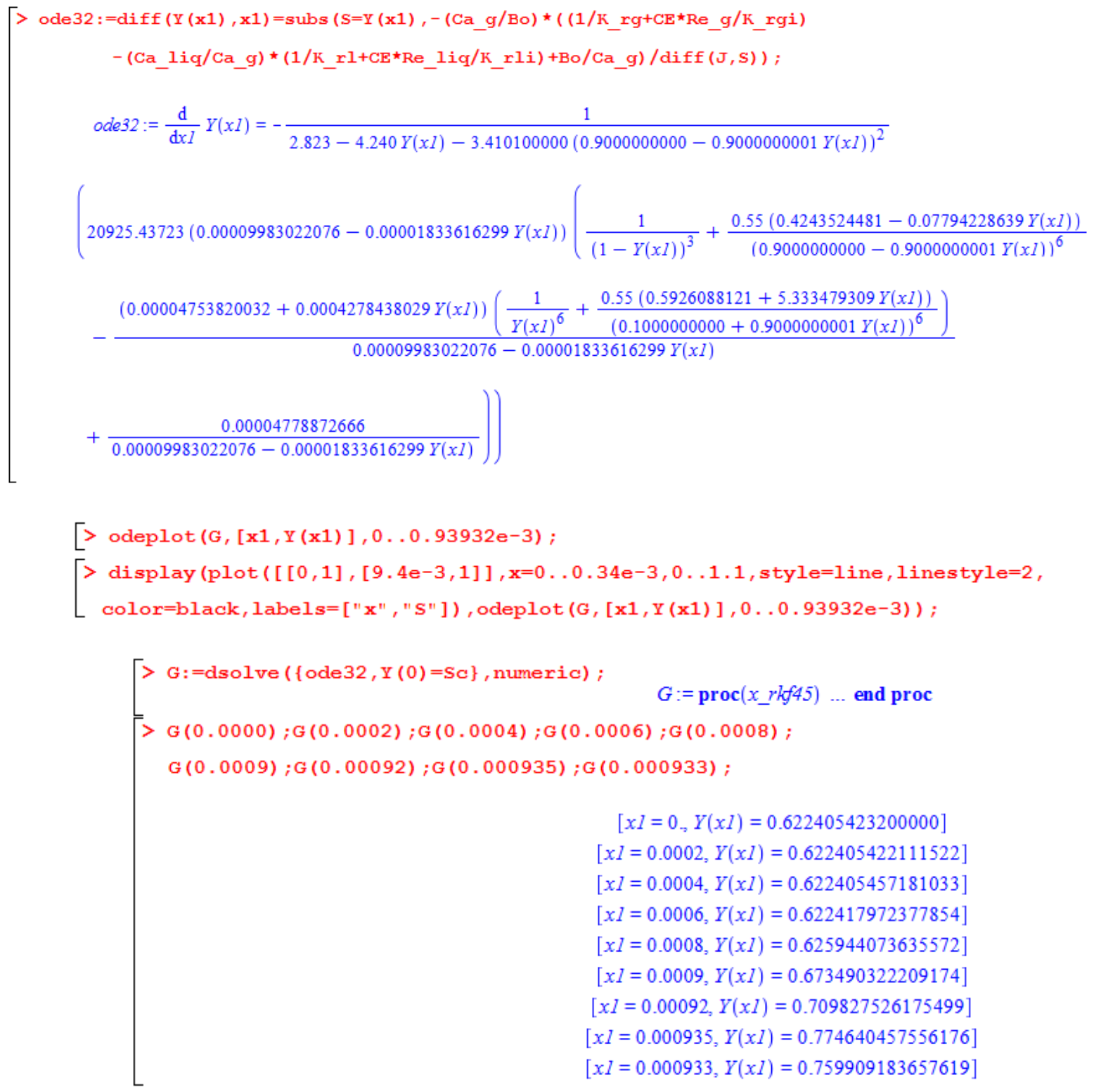




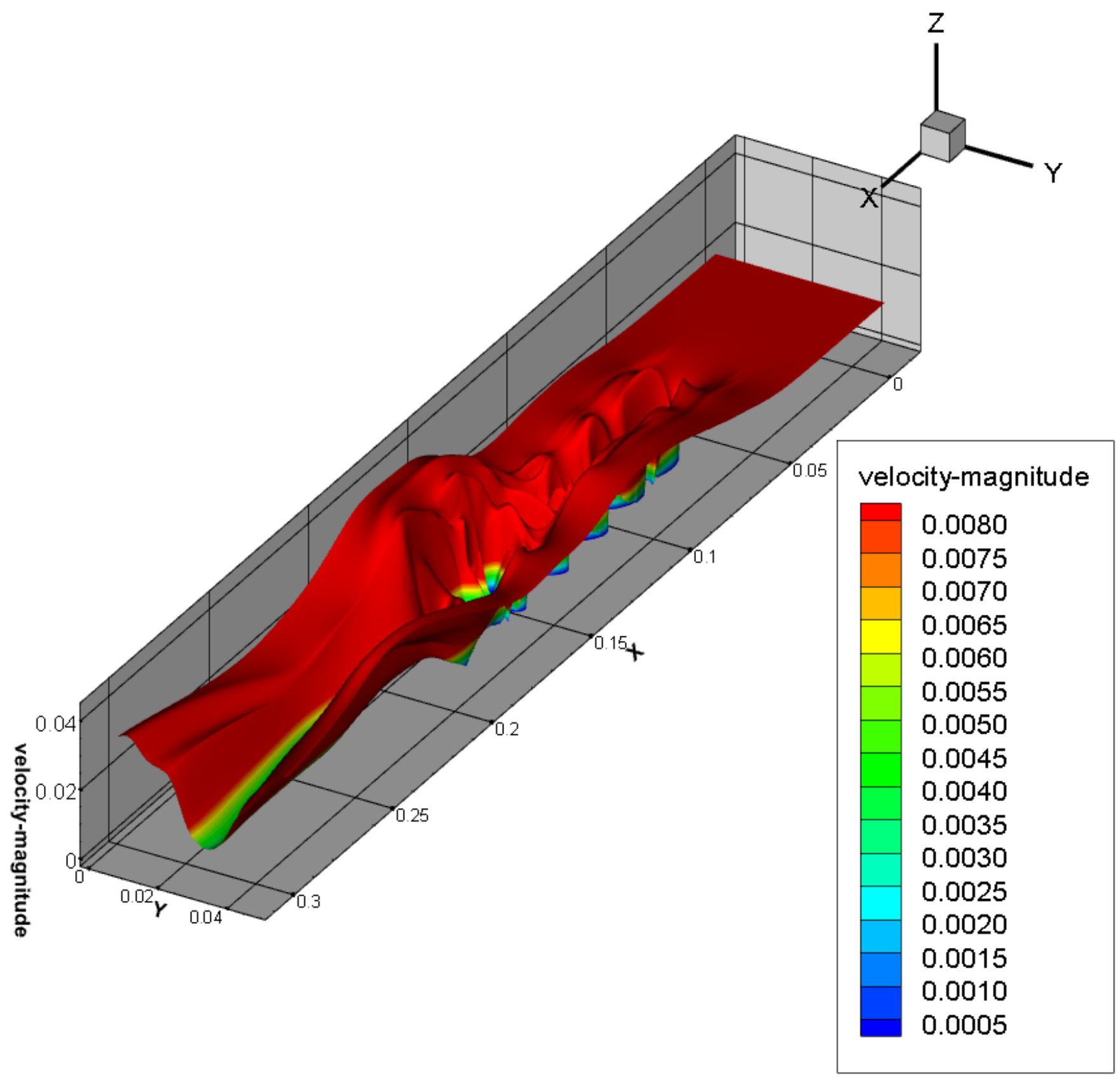

Figure C.3. Contour of Velocity Distribution 


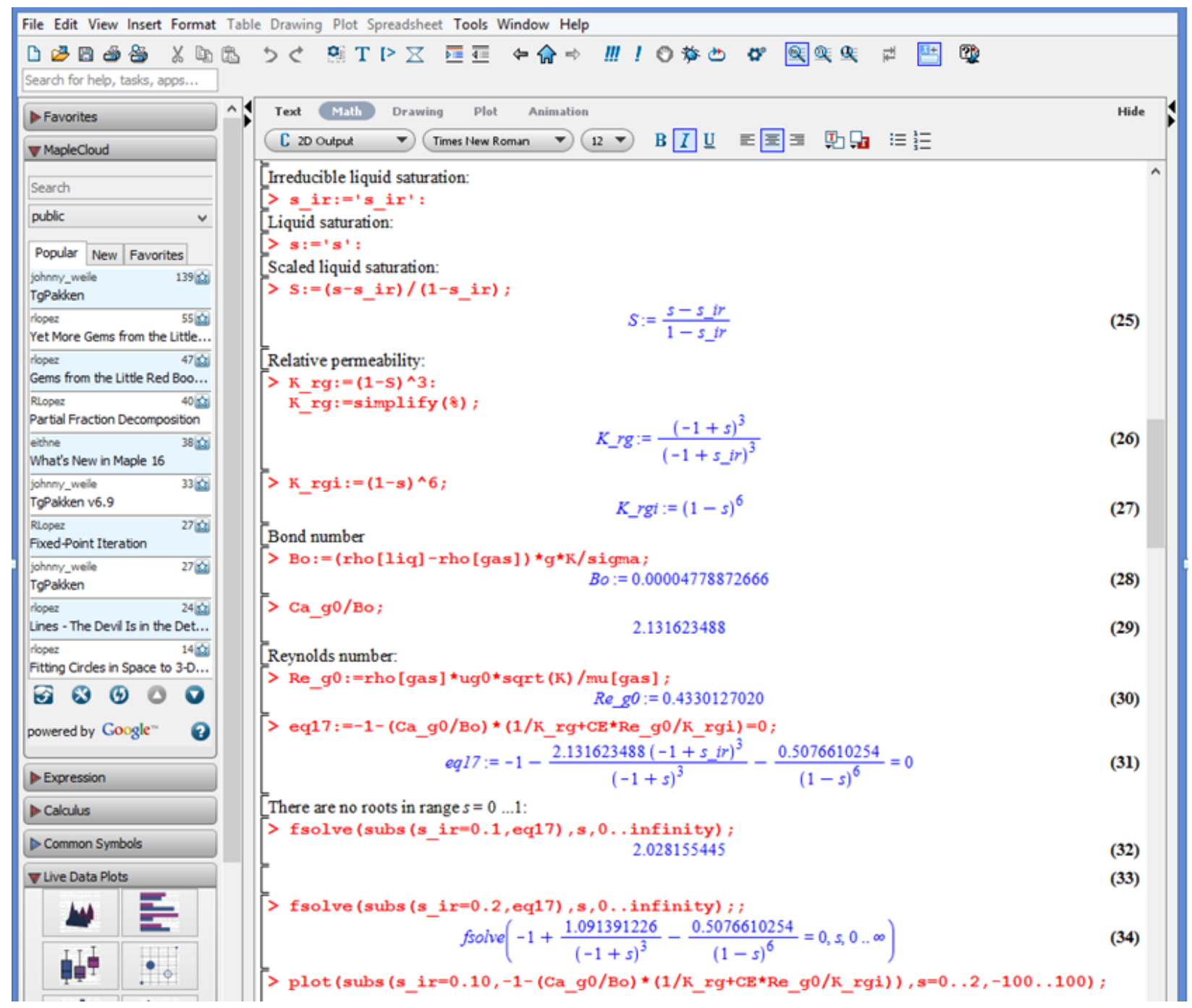




\begin{tabular}{|c|c|c|c|}
\hline \multicolumn{4}{|c|}{ File Edit View Insert Format Table Drawing Plot Spreadsheet Tools Window Help } \\
\hline 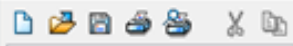 & 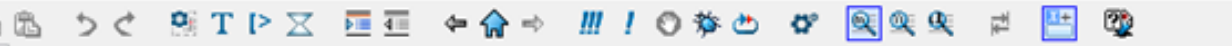 & & \\
\hline \multicolumn{4}{|l|}{ Search for help, tasks, apps... } \\
\hline$\checkmark$ Favorites & A Text Math Drawing Plot Animation & Hide & \\
\hline$\nabla$ Maplecloud & 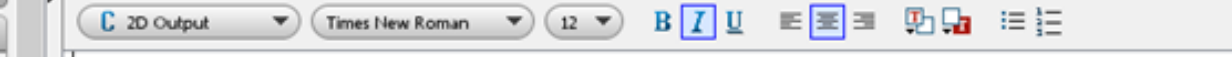 & & \\
\hline Search & P s:-'s':s:='s': & 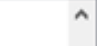 & \\
\hline public $\quad v$ & $>s$ ir: $=0.1$ & & \\
\hline \begin{tabular}{|l|l|l|} 
Popular & New & Favorites \\
\end{tabular} & $s_{-} i r:=0.1$ & (35) & \\
\hline 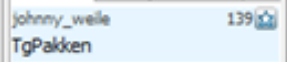 & $\begin{array}{l}\text { [Scaled critical liquid saturation Sc: } \\
{\left[>\mathrm{s}:=\mathrm{solve}\left(\mathrm{S}=\left(\mathrm{s}-\mathrm{s} \_i r\right) /\left(1-\mathrm{s} \_i r\right), \mathrm{s}\right)\right.}\end{array}$ & & \\
\hline \multirow{2}{*}{ 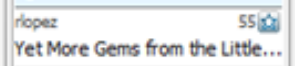 } & $\bar{s}:=0.1000000000+0.9000000001 S$ & (36) & \\
\hline & $>$ Ca_liq:=epsilon*s*uF_ast; & & \\
\hline $\begin{array}{l}47.28 \\
\text { Geper }\end{array}$ & $\overline{C a}$ lif $:=0.00004753820032+0.0004278438029 \mathrm{~S}$ & (37) & \\
\hline $\begin{array}{l}400 \text { Rat } \\
\text { Partial Fraction Decomposition }\end{array}$ & $\begin{array}{r}>\text { Ca_g: =Ca_g0-epsilon*s*uF_ast/mu [ast] } ; \\
\qquad a \_g:=0.00009983022076-0.00001833616299 \mathrm{~S}\end{array}$ & (38) & \\
\hline $\begin{array}{l}\text { ethne } \\
\text { What's New in Maple } 16\end{array}$ & 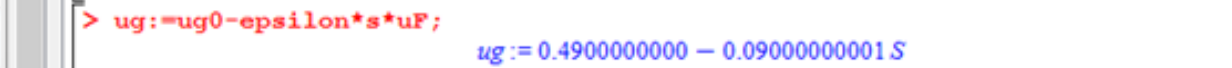 & (39) & \\
\hline 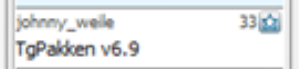 & $>$ ul: $=$ epsilon*s*uF; $\quad u l:=0.01000000000+0.09000000001 S$ & $(40)$ & \\
\hline \begin{tabular}{|l} 
Rlopez \\
Fixed Point Iteration
\end{tabular} & $>$ Re_g:=rho[gas] *ug*sqrt (K)/mu[gas] ; & & \\
\hline $\begin{array}{l}\text { johrey_wele } \\
\text { Topacken }\end{array}$ & $\begin{array}{l}\operatorname{Re} g:=0.4243524481-0.07794228639 S \\
>\operatorname{Re} 1 \text { iq }:=r h o[1 \text { iq }] * u 1 * \operatorname{sqxt}(\mathrm{K}) / \mathrm{mu}[1 \dot{i q}] ;\end{array}$ & (41) & \\
\hline 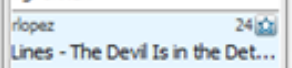 & Re_liq $:=0.5926088121+5.333479309 \mathrm{~S}$ & (42) & \\
\hline $\begin{array}{l}14 \sqrt{20} \\
\text { rlope: } \\
\text { fitting Cirdes in Space to } 3-0 . . .\end{array}$ & $K_{-} r g:=(1-S)^{3}$ & (43) & \\
\hline $\begin{array}{llll}3 & 8 & 0 & 0 \\
\text { powered by Google- } & 2\end{array}$ & 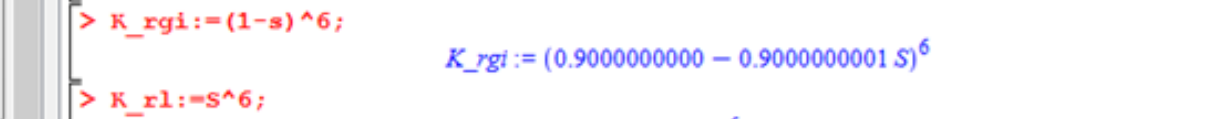 & (44) & \\
\hline Expression & $K_{-} r l:=S^{6}$ & (45) & \\
\hline$\nabla$ Calaus & $K_{-}$rli $:=(0.1000000000+0.9000000001 S)^{6}$ & (46) & \\
\hline$D$ Common Symbols & $>$ eq32num: $=\left(1 / \mathrm{K} \_r g+C E \star R e \_g / K \_r g i\right)-\left(C a \_1 i q / C a \_g\right) \star\left(1 / K \_r l+C E \star R e \_1 i q / K \_r l i\right)+$ & & \\
\hline$\nabla$ Live Data Plots & Bo/Ca_g=0 ; $\quad 0.55(0.4243524481-0.07794228639 S)$ & & \\
\hline 4 플 & eq32non $:=\frac{1}{(1-S)^{3}}+\frac{0.9000000000-0.9000000001 S)^{6}}{(0.90}$ & (47) & \\
\hline 旦夏 & $(0.00004753820032+0.0004278438029 S)\left(\frac{1}{S^{6}}+\frac{0.55(0.5926088121+5.333479309 S)}{(0.1000000000+0.9000000001 S)^{6}}\right)$ & & \\
\hline & $0.00009983022076-0.00001833616299 S$ & & \\
\hline 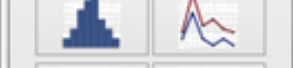 & $+\frac{0.00004778872666}{0.00009983022076-0.00001833616299 S}=0$ & & \\
\hline$\theta$ & $\begin{array}{r}\text { > Sc: }=\text { fsolve (eq32num, s, -infinity...infinity) } \\
\text { Sc }:=0.6224054232\end{array}$ & (48) & \\
\hline
\end{tabular}




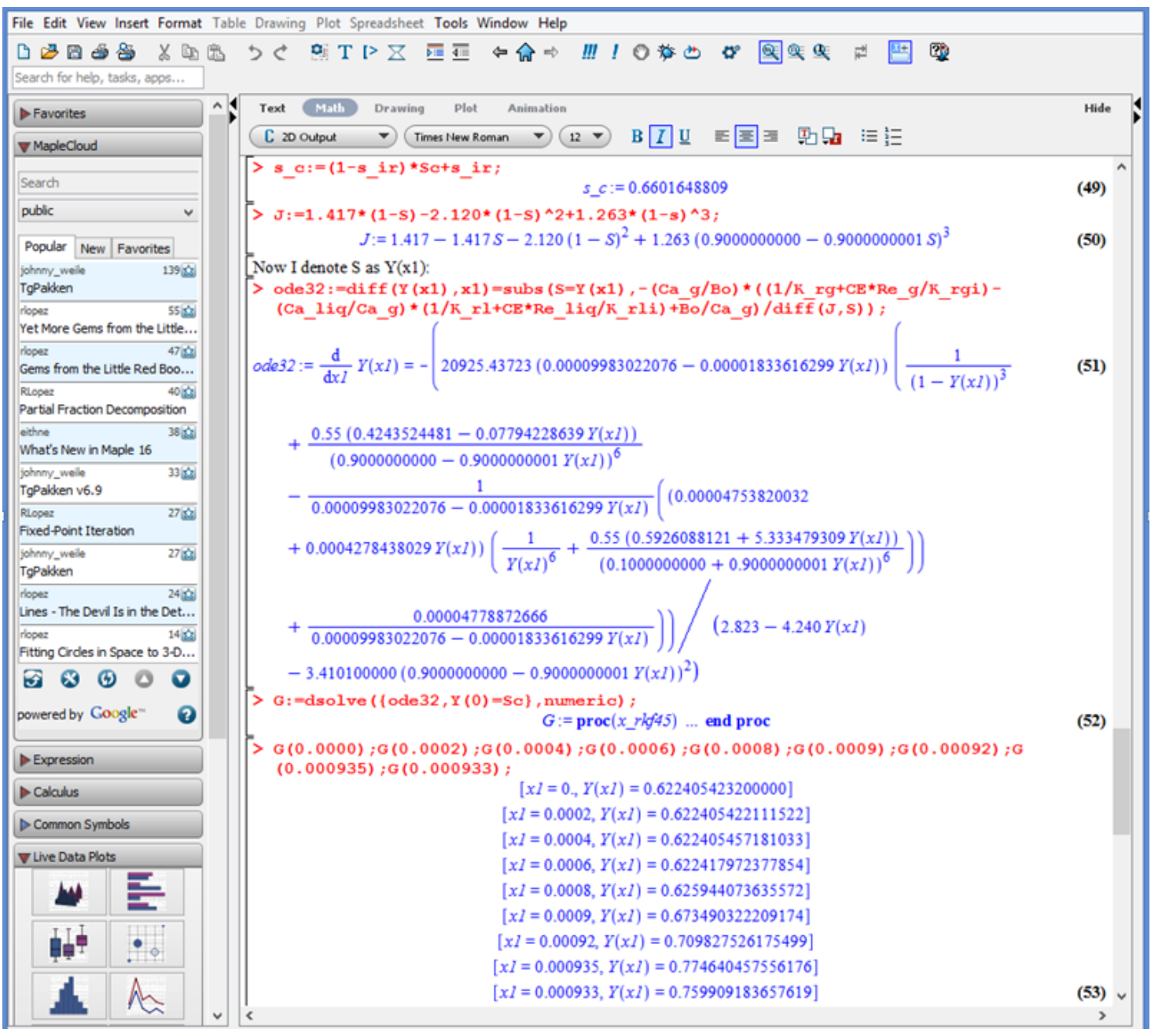

\section{C3: HEAT SINK EXPERIMENTAL DESIGN CACULATIONS}

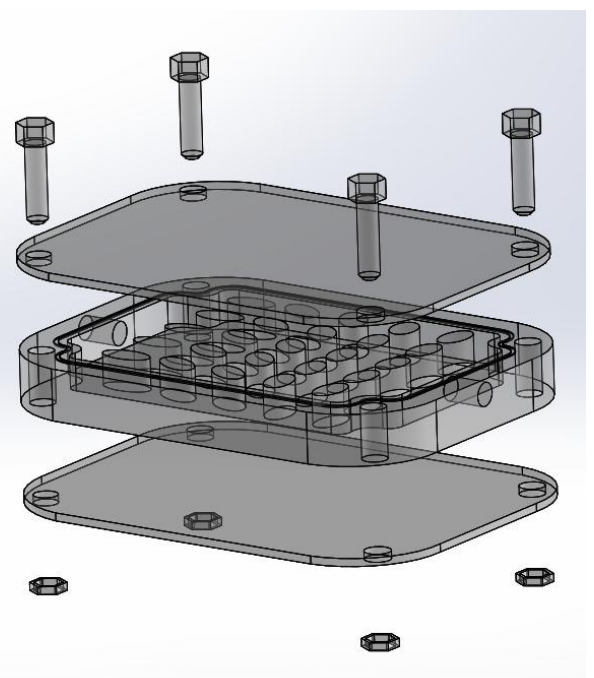

C4. Four bolts to tighten lexan sheets 


\section{DESIGN CALCULATIONS (I):}

Fin, finite length, heat flow from conductive material, $Q=\left(K^{*} A_{b} * n^{*} \Delta T\right) * X_{1} / X_{2}$

Inlet Temperatrue, $T_{i n}=70^{\circ} \mathrm{F}=21.11^{\circ} \mathrm{C}$

Outlet Temperature, $T_{\text {out }}=80^{\circ} \mathrm{F}=26.66^{\circ} \mathrm{C}$

Fin Diameter, $D=3.0012939 \mathrm{~mm}$

Fin Material Thermal Conductivity, $K=401 \mathrm{~W} / \mathrm{m}^{\circ} \mathrm{C}$

Convective Heat Transfer Coeffecient, $h=57.2 \mathrm{~W} / \mathrm{m}^{2}{ }^{\circ} \mathrm{C}$

Fin Height, $H=7.2 \mathrm{~mm}$

Number of Fins, $N=25$

Fin Base Area, $A=\pi D^{2} / 4 \mathrm{~mm}^{2}$

Temperatrue difference, $\Delta T=T_{1}-T_{2}, \Delta T=5.55^{\circ} \mathrm{C}$

For Fin, $n=(2 h / K * \mathrm{~d})^{0.5}$

$n=9.7517$

$(h / n * K)=0.005706$

$H=0.007 \mathrm{~m}$

$n * H=0.068262 m, \sinh (n * H)=0.067052, \cosh (n * H)=1.00225$

$X_{1}=[(\sinh (n * L))+(h / n * K) * \cosh (n * L)], X_{1}=0.0041$

$X_{2}=[(\cosh (n * L))+(h / n * K) * \sinh (n * L)], X_{2}=1.0112$

$Q=N *\left(K^{*} A^{*} n^{*} \Delta T\right)\left(X_{1} / X_{2}\right)$

$Q=217.5 \mathrm{Watts}$

Heat flow rate from forced convection,

$Q=\dot{m} c_{p}\left(T_{\text {out }}-T_{\text {in }}\right)$

$\dot{m}=V \rho_{l} A, \dot{m}=1.0 x 980\left(\frac{0.003}{2}\right)^{2} \pi, \dot{m}=0.006927 \mathrm{~kg} / \mathrm{s}$

$Q=0.006927 x 4200(5.55)=145.07$ Watts,$Q=161.468$ Watts

$Q=217.5$ Watts +161.468 Watts $=378.968$ Watts 


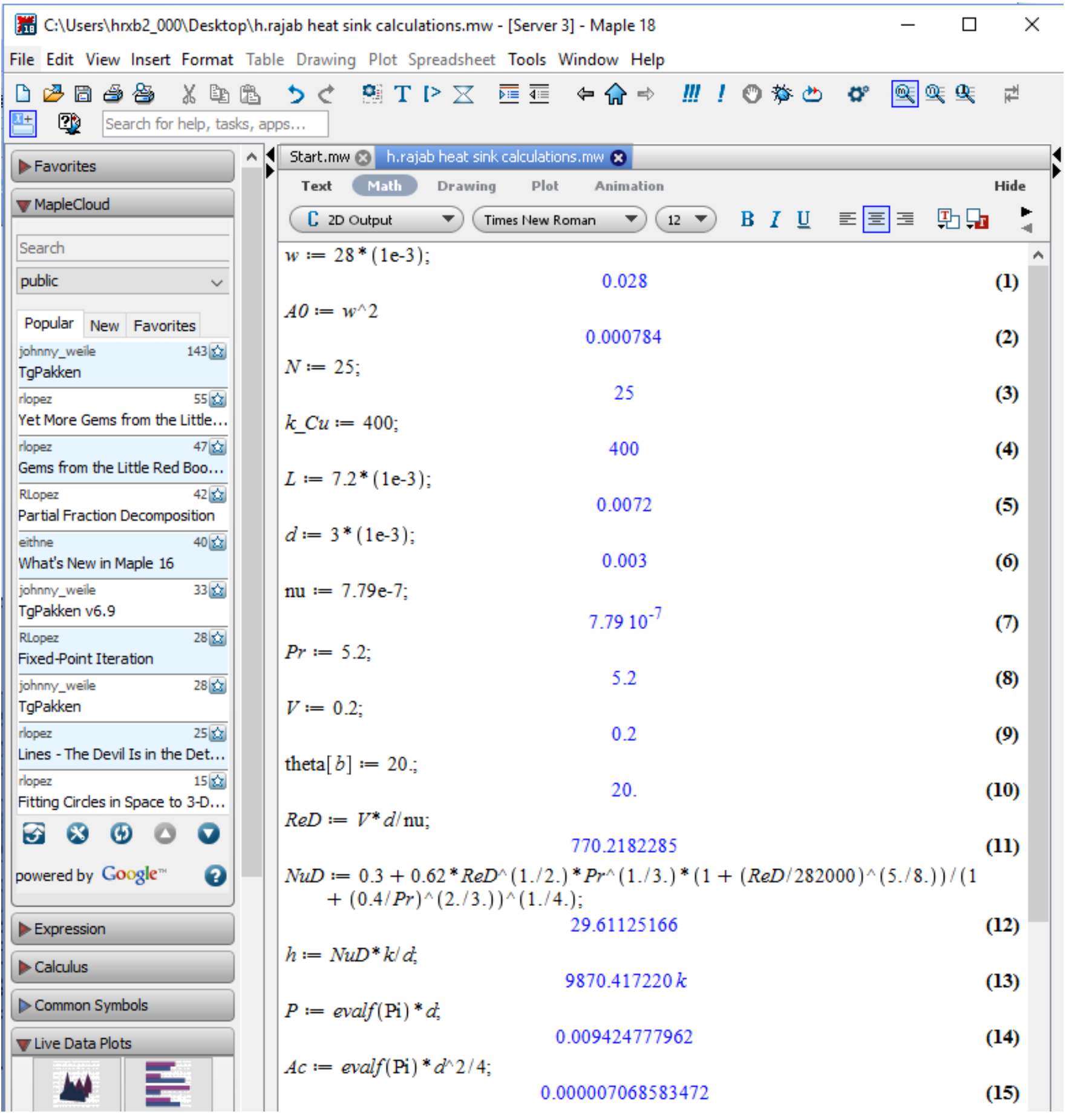


19: C: \Users \hrob2_000\Desktop \h.rajab heat sink calculations.mw - [Server 3] - Maple 18

File Edit View Insert Format Table Drawing Plot Spreadsheet Tools Window Help

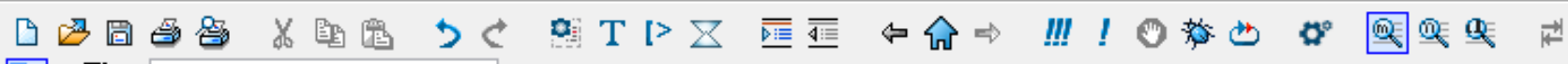

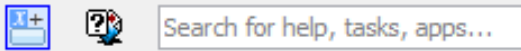

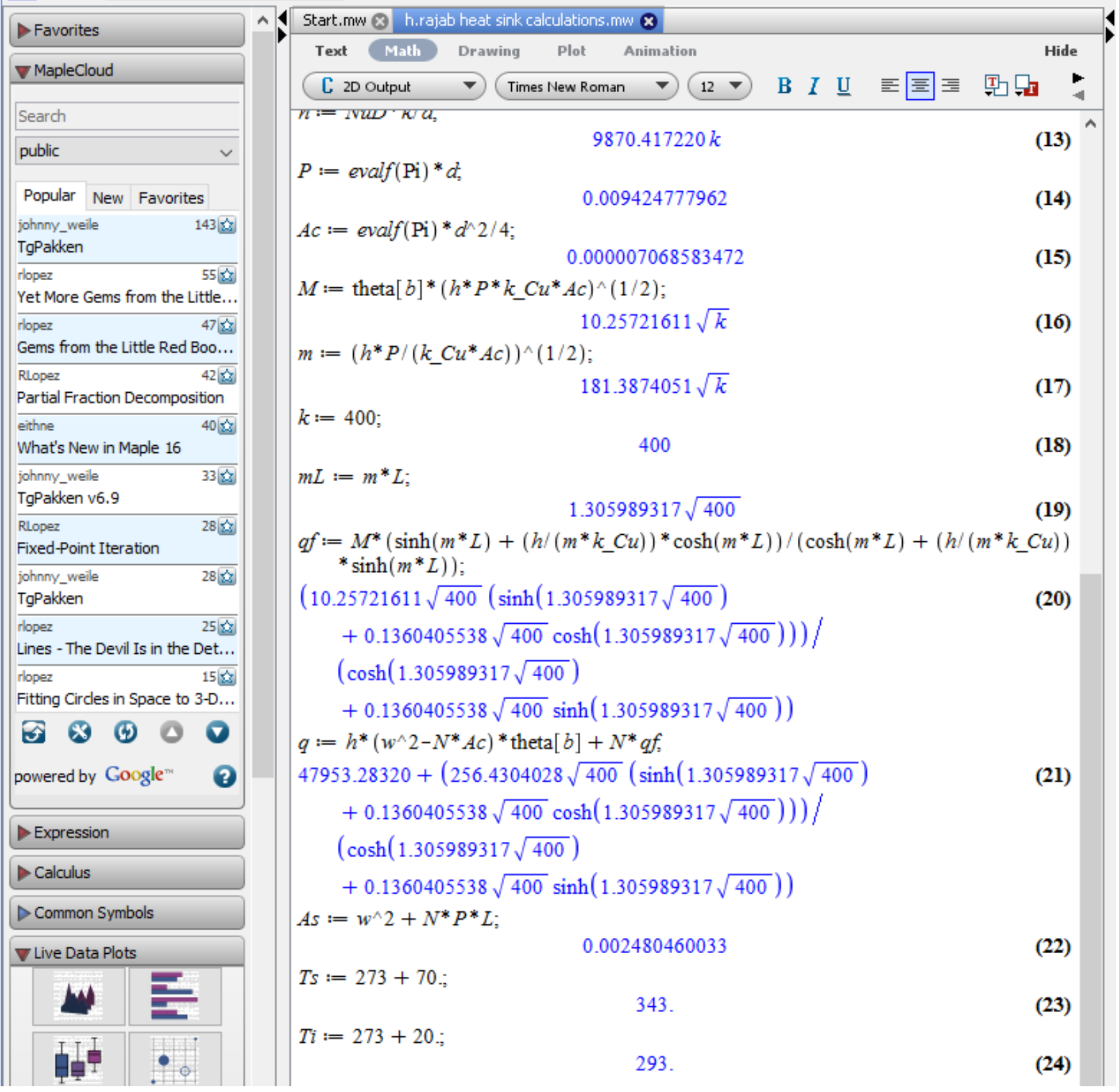




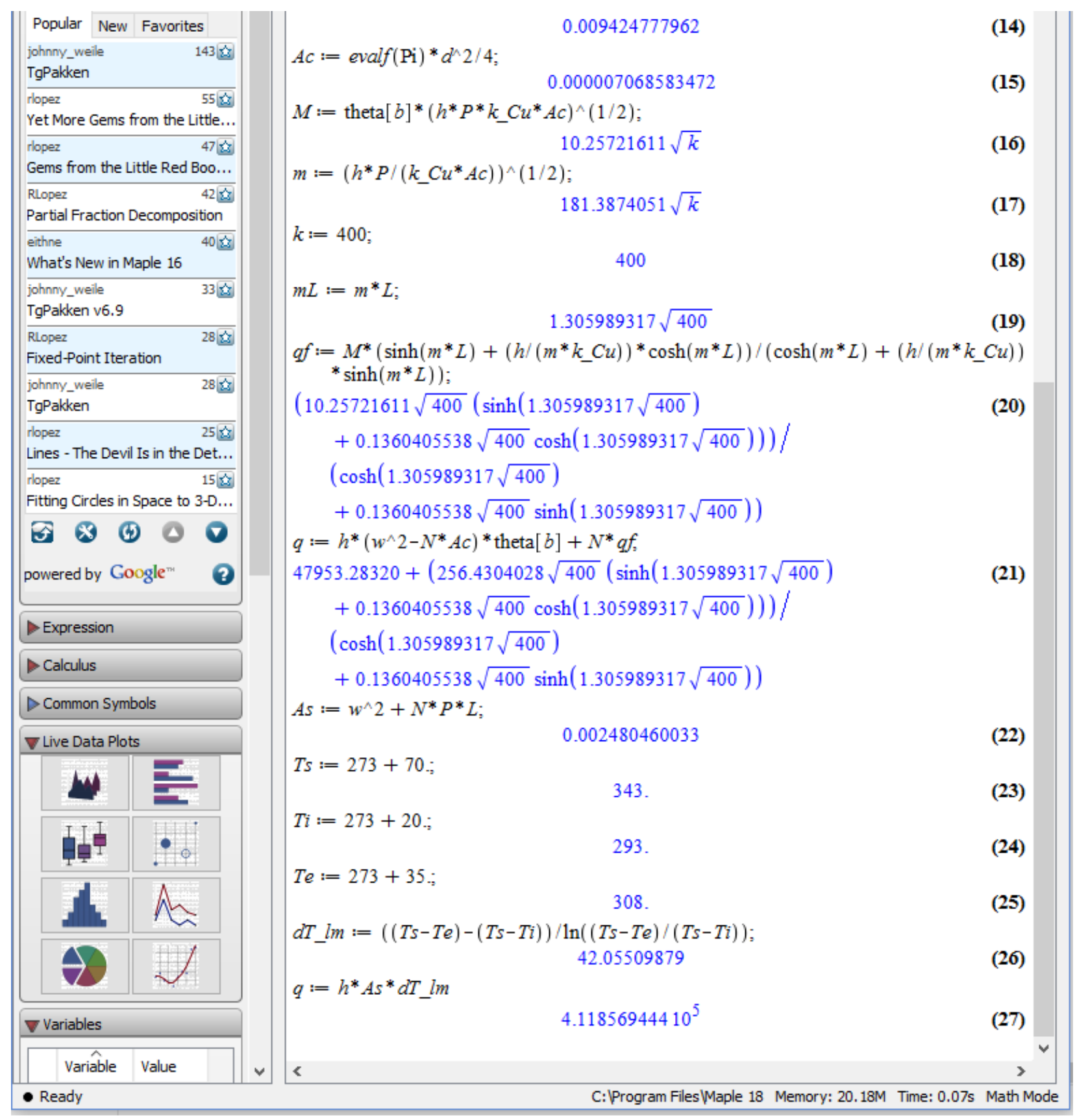




\section{DESIGN CALCULATIONS (II):}

Fin, finite length, heat flow from conductive material, $Q=\left(K^{*} A_{b} * n * \Delta T\right) * X_{1} / X_{2}$

Inlet Temperatrue, $T_{i n}=70^{\circ} \mathrm{F}=21.11^{\circ} \mathrm{C}$

Outlet Temperature, $T_{\text {out }}=80^{\circ} \mathrm{F}=26.66^{\circ} \mathrm{C}$

Fin Diameter, $D=3.0012939 \mathrm{~mm}$

Fin Material Thermal Conductivity, $K=401 \mathrm{~W} / \mathrm{m}^{\circ} \mathrm{C}$

Convective Heat Transfer Coeffecient, $h=57.2 \mathrm{~W} / \mathrm{m}^{2}{ }^{\circ} \mathrm{C}$

Fin Height, $H=7.2 \mathrm{~mm}$, Number of Fins, $N=25$

Fin Base Area, $A=\pi D^{2} / 4 \mathrm{~mm}^{2}$

Temperatrue difference, $\Delta T=T_{1}-T_{2}, \Delta T=5.55^{\circ} \mathrm{C}$

For Fin, $n=(2 h / K * \mathrm{~d})^{0.5}, n=9.7517$

$\left(h / \mathrm{n}^{*} K\right)=0.005706, H=0.007 m$

$n^{*} H=0.068262 m, \sinh \left(n^{*} H\right)=0.067052, \cosh (n * H)=1.00225$

$X_{1}=[(\sinh (n * L))+(h / n * K) * \cosh (n * L)], X_{1}=0.0041$

$X_{2}=[(\cosh (n * L))+(h / n * K) * \sinh (n * L)], X_{2}=1.0112$

$Q=N *\left(K^{*} A * n * \Delta T\right)\left(X_{1} / X_{2}\right), Q=217.5$ Watts

Heat flow rate from forced convection, $Q=\dot{m} c_{p}\left(T_{\text {out }}-T_{\text {in }}\right)$

$\dot{m}=V \rho_{l} A, \dot{m}=1.7 x 980\left(\frac{0.004}{2}\right)^{2} \pi, \dot{m}=0.020936 \mathrm{~kg} / \mathrm{s}$

$Q=0.020936 \times 4200(5.55)=488.008$ Watts,$Q=488.008$ Watts

$Q=217.5$ Watts +488.008 Watts $=705.508$ Watts 


\section{DESIGN CALCULATIONS (III):}

Fin, finite length, heat flow from conductive material, $Q=\left(K^{*} A_{b} * n * \Delta T\right) * X_{1} / X_{2}$

Inlet Temperatrue, $\quad T_{i n}=70^{\circ} \mathrm{F}=21.11^{\circ} \mathrm{C}$

Outlet Temperature, $T_{\text {out }}=80^{\circ} \mathrm{F}=26.66^{\circ} \mathrm{C}$

Fin Diameter, $D=3.0012939 \mathrm{~mm}$

Fin Material Thermal Conductivity, $K=401 \mathrm{~W} / \mathrm{m}^{\circ} \mathrm{C}$

Convective Heat Transfer Coeffecient, $h=57.2 \mathrm{~W} / \mathrm{m}^{2}{ }^{\circ} \mathrm{C}$

Fin Height, $H=7.2 \mathrm{~mm}$, Number of Fins, $N=25$

Fin Base Area, $A=\pi D^{2} / 4 \mathrm{~mm}^{2}$

Temperatrue difference, $\Delta T=T_{1}-T_{2}, \Delta T=5.55^{\circ} \mathrm{C}$

For Fin, $n=(2 h / K * \mathrm{~d})^{0.5}, n=9.7517$

$\left(h / \mathrm{n}^{*} K\right)=0.005706, H=0.007 m$

$n^{*} H=0.068262 m, \sinh (n * H)=0.067052, \cosh (n * H)=1.00225$

$X_{1}=[(\sinh (n * L))+(h / n * K) * \cosh (n * L)], X_{1}=0.0041$

$X_{2}=[(\cosh (n * L))+(h / n * K) * \sinh (n * L)], X_{2}=1.0112$

$Q=N *(K * A * n * \Delta T)\left(X_{1} / X_{2}\right), Q=217.5$ Watts

Heat flow rate from forced convection, $Q=\dot{m} c_{p}\left(T_{\text {out }}-T_{\text {in }}\right)$

$$
\begin{aligned}
& \dot{m}=V \rho_{l} A, \dot{m}=1.8 x 980\left(\frac{0.004}{2}\right)^{2} \pi, \dot{m}=0.022167077 \mathrm{~kg} / \mathrm{s} \\
& Q=0.022167077 x 4200(5.55)=516.715 \text { Watts }, Q=516.715 \text { Watts } \\
& Q=217.5 \text { Watts }+516.715 \text { Watts }=734.215 \text { Watts }
\end{aligned}
$$




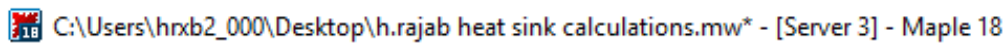

File Edit View Insert Format Table Drawing Plot Spreadsheet Tools Window Help

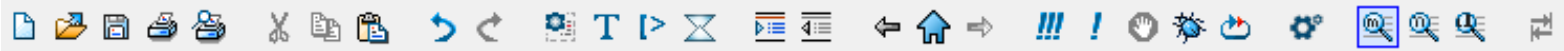

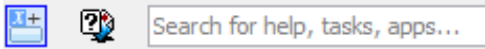

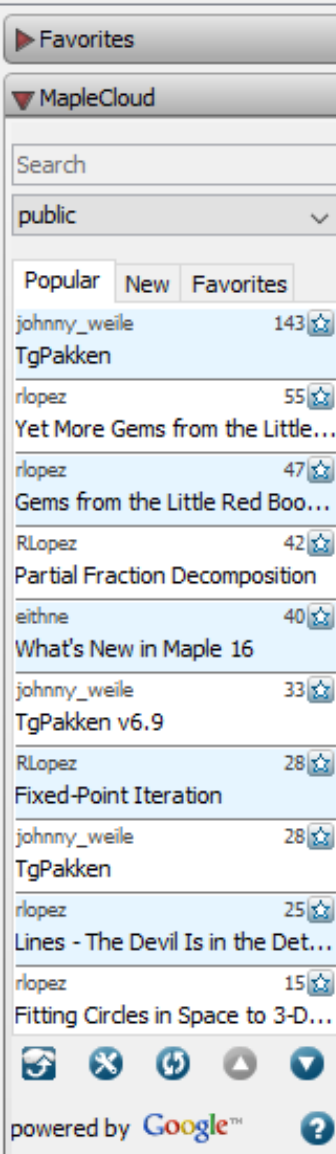

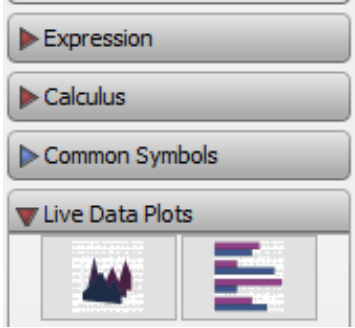

\section{$\wedge$ Start.mw $\mathbb{\otimes}$ *h.rajab heat sink calculations.mw $\mathbf{x}$}

Text Math Drawing Plot Animation

C 2D Math $\nabla$

Times New Roman

$12 \nabla$

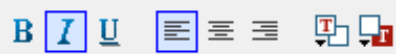

$w:=40 *(1 \mathrm{e}-3)$;

0.040

(1)

$A O:=w^{\wedge} 2$

0.001600

(2)

$N:=25$

25

$k \_C u:=400$;

400

$L:=10.2 *(1 \mathrm{e}-3)$

0.0102

$d:=1.5^{*}(1 \mathrm{e}-3)$

0.0015

$\mathrm{nu}:=7.79 \mathrm{e}-7$;

$7.7910^{-7}$

$\operatorname{Pr}:=5.2$

$V:=0.5$

theta $[b]:=20$;

20.

$\operatorname{ReD}:=V^{*} d / \mathrm{nu}$;

962.7727856

(11)

$N u D:=0.3+0.62 * \operatorname{Re} D^{\wedge}(1 . / 2 .)^{*} \operatorname{Pr}^{\wedge}(1 . / 3 .)^{*}\left(1+(\operatorname{ReD} / 282000)^{\wedge}(5 . / 8).\right) /(1$ $\left.+(0.4 / P r)^{\wedge}(2 . / 3).\right)^{\wedge}(1 . / 4$.$) ;$

33.19053927

(12)

$h:=N u D^{*} k / d^{2}$,

$P:=\operatorname{evalf}(\mathrm{Pi}) * d$,

$$
8.85081047310^{6}
$$

$$
0.004712388981
$$

$A c:=\operatorname{evalf}(\mathrm{Pi}) * d^{\wedge} 2 / 4$ 


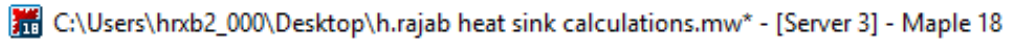

File Edit View Insert Format Table Drawing Plot Spreadsheet Tools Window Help

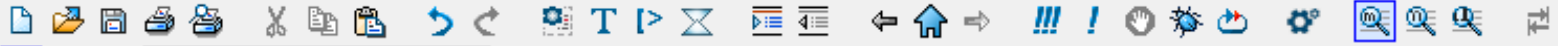

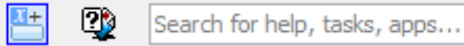

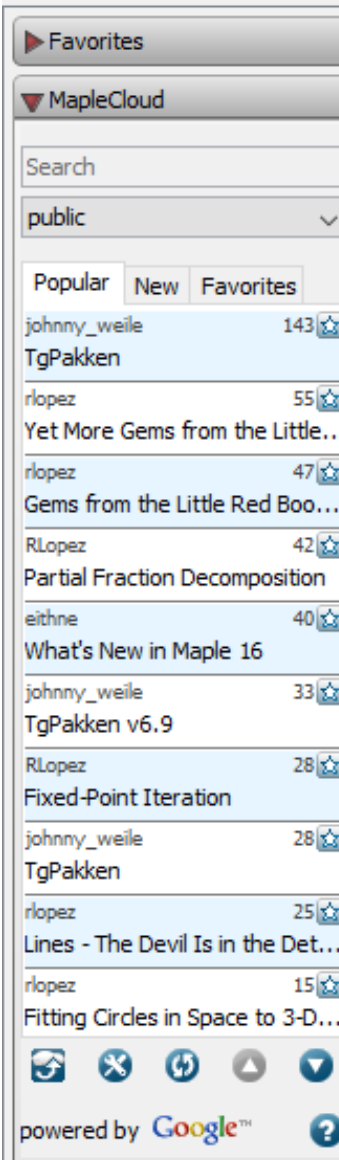

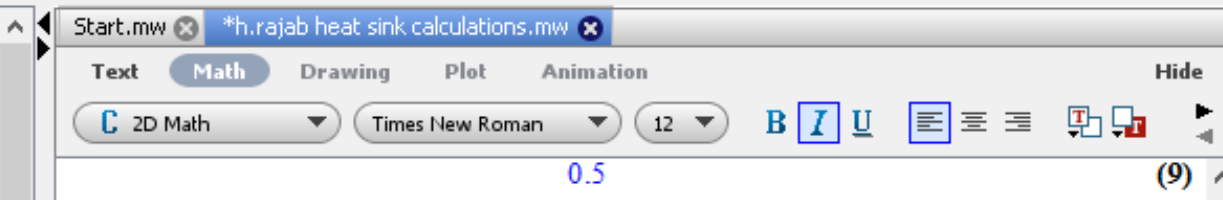

theta $[b]:=20$;

$\operatorname{ReD}:=V^{*} d / \mathrm{nu}$

20.

962.7727856

(11)

$N u D:=0.3+0.62^{*} \operatorname{Re} D^{\wedge}(1 . / 2 .)^{*} \operatorname{Pr}^{\wedge}(1 . / 3 .)^{*}\left(1+(\operatorname{ReD} / 282000)^{\wedge}(5 . / 8).\right) /(1$ $\left.+(0.4 / P r)^{\wedge}(2 . / 3).\right)^{\wedge}(1 . / 4$.$) ;$

33.19053927

$h:=N u D^{*} k / d^{2}$

$8.85081047310^{6}$

$P:=\operatorname{evalf}(\mathrm{Pi}) * d$,

0.004712388981

(13)

$A c:=\operatorname{evalf}(\mathrm{Pi}) * d^{\wedge} 2 / 4$

0.000001767145868

(14)

$M:=\operatorname{theta}[b]^{*}\left(h^{*} P^{*} k \_C u^{*} A c\right)^{\wedge}(1 / 2)$;

108.5946119

(15)

$m:=\left(h^{*} P /\left(k_{-} C u^{*} A c\right)\right)^{\wedge}(1 / 2) ;$

7681.497456

(16)

$k:=400$;

400

(17)

$m L:=m^{*} L$

78.35127405

$q f:=M^{*}\left(\sinh \left(m^{*} L\right)+\left(h /\left(m^{*} k_{-} C u\right)\right) * \cosh \left(m^{*} L\right)\right) /\left(\cosh \left(m^{*} L\right)+\left(h /\left(m^{*} k_{-} C u\right)\right)\right.$ $\left.* \sinh \left(m^{*} L\right)\right)$;

\section{Expression}

Calculus

$D$ Common Symbols

$\nabla$ Live Data Plots

108.5946119

(20)

$q:=h^{*}\left(w^{\wedge} 2-N^{*} A c\right) *$ theta $[b]+N^{*} q f$,

$2.78120463910^{5}$

(21)

$A s:=w^{\wedge} 2+N^{*} P^{*} L$

0.002801659190

(22)

$T s:=273+50$;

323.

(23) 


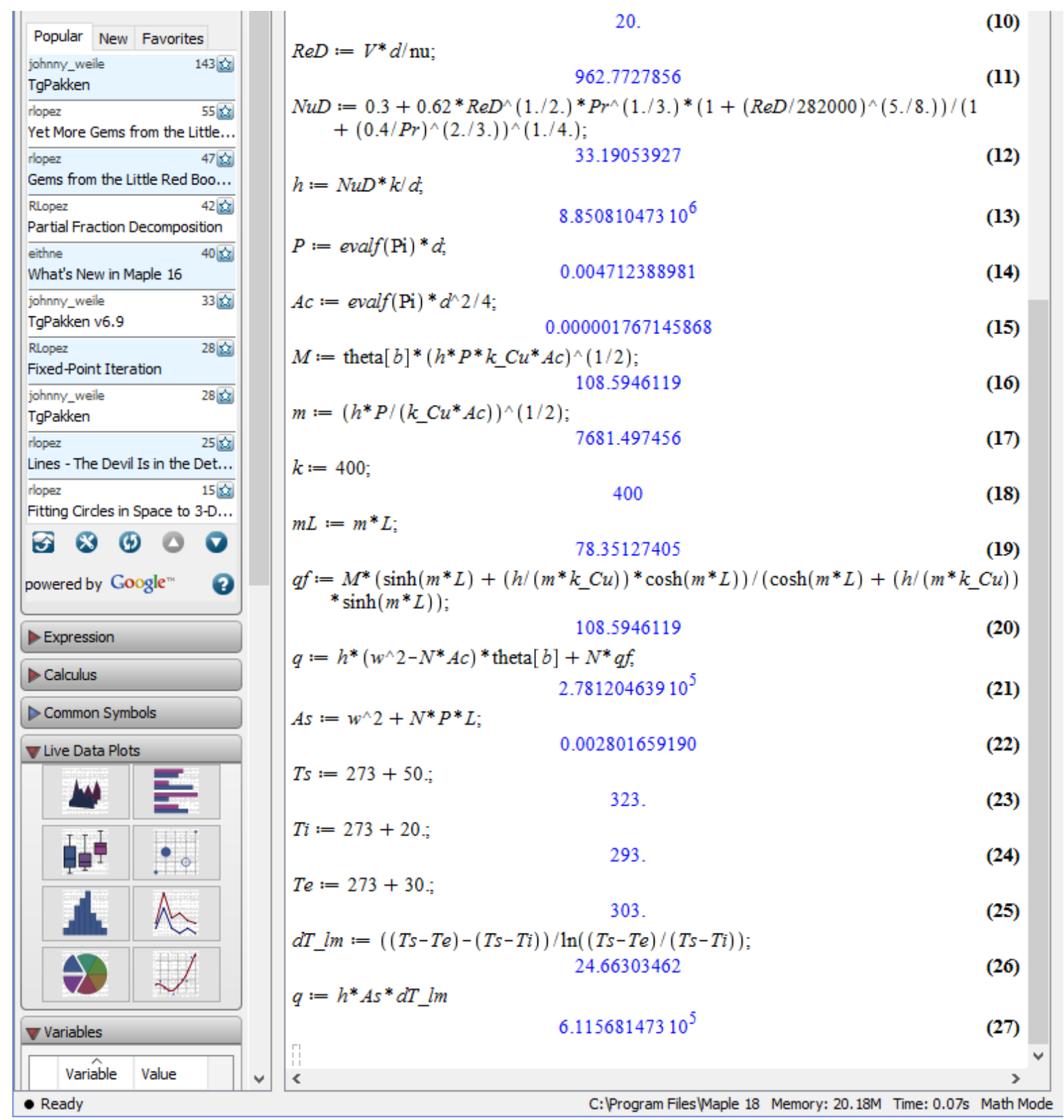




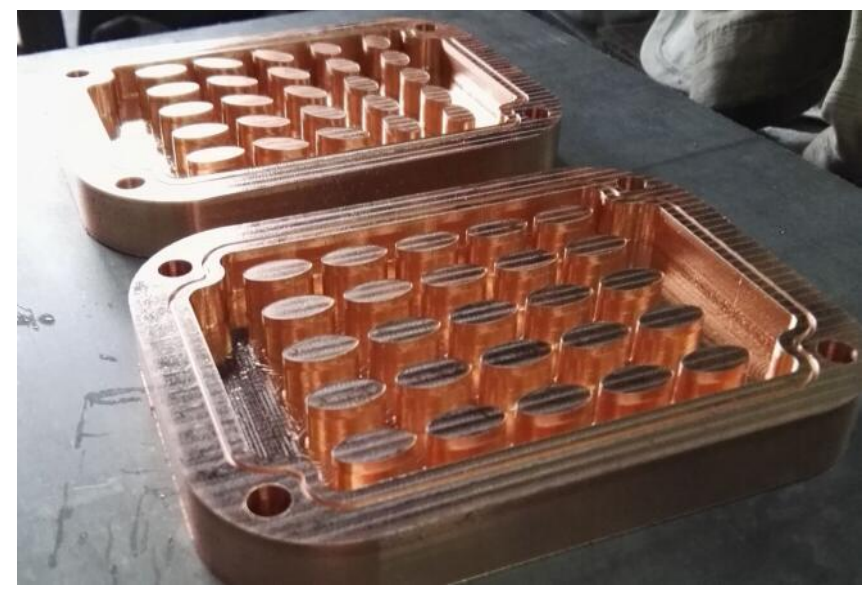

Figure C.5. Photograph of prototype II

\section{REFERENCES}

1. Ma, H.B., Wilson, C., Borgmeyer, B., Park, K., Yu, Q., Choi,S. U. S. and Tirumala, M., (2006) Effect of Nanofluid on The Heat Transport Capability in an Oscillating Heat Pipe, Applied Physical Letters 88143116.

2. Ma, H.B., Wilson, C., Yu, Q., Park, K., Choi,S. U. S. and Tirumala, M., (2006) An Experimental Investigation of Heat Transport Capability in a Nano-Fluid Oscillating Heat Pipe, ASME Journal of Heat Transfer, 128, pp. 1213-1216.

3. Wilson, C., Ma, H. B., Yu, Q. and Park, P., (2006) High Thermal Conductivity of Diamond Nanofluid and Its Effect on the Heat Transport Capability in an Oscillating Heat Pipe, Proc. of IMECE, ASME Intl. Mechanical Engineering Congress and Exposition, Chicago, IL, pp. 289-295.

4. Rajab, H., Yin, D., \& Ma, H. B. (2017). Numerical analysis of effects of nanofluid and angular orientation on heat transfer performance of an elliptical pin-fin heat sink. Heat Transfer Research, 48(2), 161-175. doi:10.1615/HeatTransRes.2016011084.

5. Rajab, H., Yin, D., \& Ma, H. (2014). Effects of Al2O3-water nanofluid and angular orientation on entropy generation and convective heat transfer of an elliptical micro-pin- 
fin heat sink. Paper presented at the ASME International Mechanical Engineering Congress and Exposition, Proceedings (IMECE), 8A, doi:10.1115/IMECE2014-40335.

6. Yu, W., France, D. M., Choi, S. U. S., and Routbort, J. L., (2007) Review and Assessment of Nanofluid Technology for Transportation and Other Applications, Argonne National Laboratory, Technical Report (78), Report No. ANL/ESD/07-9.

7. Godson, L., Raja, B., Lal, D. and Wongwises, S., (2010) Enhancement of Heat Transfer Using Nanofluids- An Overview, Renewable Sustainable Energy Rev., 14 pp. 629-641.

8. Anoop, K.B., Sundararajan, T. and Das, S.K., (2009) Effect of Particle Size on the Convective Heat Transfer in Nanofluid in the Developing Region, International Journal of Heat and Mass Transfer, 52 (9-10) pp. 2189-2195.

9. Wang, X.Q. and Mujumdar, A.S., (2007) Heat Transfer Characteristics of Nanofluids: A Review, International Journal of Thermal Science, 46 pp. 1-19.

10. Hamilton, R. L. and Crosser, O. K., (1962) Thermal Conductivity of Heterogeneous Two Component Systems, I \& EC Fundamentals I, pp. 187-191.

11. Maxwell, J.C., (1904) A Treatise on Electricity and Magnetism, second ed. Oxford University Press, Cambridge, UK.

12. Koo, J. and Kleinstreuer, C., (2004) A new thermal conductivity model for nanofluids, Journal of Nanoparticle Research, (6) pp. 577-588.

13. Prasher, R. S., Bhattacharya, P. and Phelan, P. E., (2005) Thermal Conductivity of Nanoscale Colloidal Solutions (Nanofluids), Physical Review Letters, (94) 025901.

14. Evans, W., Fish, J. and Keblinski, P., (2006) Role of Brownian Motion Hydrodynamics on Nanofluid Thermal Conductivity, Applied Physical Letters, 88093116. 
15. Namburu, P. K., Kulkarni, D. P., Dandekar, A. and Das, D. K., (2007) Experimental Investigation of Viscosity and Specific Heat of Silicon Dioxide Nanofluids, Micro \& Nano Letters, 2 (3) pp. 67-71.

16. Vajjha, R.S. and Das, D.K., (2009) Experimental Determination of Thermal Conductivity of Three Nanofluids and Development of New Correlations, International Journal of Heat and Mass Transfer, 52 pp. 4675-4682.

17. Wang, B. X., Zhou, L. P. and Peng, X. F., (2003) A Fractal Model for Predicting The Effective Thermal Conductivity of Liquid with Suspension of Nanoparticles, International Journal of Heat and Mass Transfer, 46 (14) pp. 2665-2672.

18. Khanafer, K. and Vafai, K., (2011) A Critical Synthesis of Thermophysical Characteristics of Nanofluids, Int. J. Heat and Mass Transfer (54) pp. 4410-4428.

19. Sparrow, E. M. and Vemuri, S. B., (1986) Orientation Effects on Natural Convection/Radiation Heat Transfer from Pin-Fin Arrays, International Journal of Heat Mass Transfer, 29(3) pp. 359-368.

20. Rubio-Jimenez, Carlos A., Kandlikar, Satish G. and Hernandez-Guerrero, Abel, (2012) Numerical Analysis of Novel Micro Pin Fin Heat Sink With Variable Fin Density, IEEE Transactions on Components, Packaging and Manufacturing Technology, 2 (5).

21. Huang, R.T. Sheu, W.J. and Wang, C.C., (2008) Orientation Effect on Natural Convective Performance of Square Pin Fin Heat Sinks, International Journal of Heat Mass Transfer, 51 (9-10) 2368-2376.

22. Zukauskas, A., (1987) Heat Transfer from Tubes in Crossflow, Advances in Heat Transfer, J. P. Hartnett and Th. F. Irvine, eds., Academic, New York, Vol.18. 
23. Xuan, Y. and Roetzel, W., (2000) Conceptions for Heat Transfer Correlations of Nanofluids, International Journal of Heat and Mass Transfer, 43 pp. 3701-3707.

24. Pak, B. C. and Cho, Y. I., (1998) Hydrodynamic and Heat Transfer Study of Dispersed Fluids With Submicron Metallic Oxide Particle, Exp. Heat Transfer, 11 pp. 151-170.

25. Koo, J. and Kleinstreuer, C. (2005) Laminar Nanofluid Flow in Microheat-Sinks, International Journal of Heat and Mass Transfer, 48 pp. 2652-2661.

26. Koo, J. and Kleinstreuer, C., (2004) A New Thermal Conductivity Model for Nanofluids, Journal of Nanoparticle Research, 6 pp. 577-588.

27. Li, J. and Kleinstreuer, C., (2008) Thermal Performance of Nanofluid Flow in Microchannels, International Journal of Heat Fluids Flow, 29 pp. 1221-1232.

28. Li, J., (2008) Computational Analysis of Nanofluid Flow in Microchannels with Applications to Micro-heat Sinks and Bio-MEMS, Ph.D. dissertation, MAE department, NCSU, Raleigh, NC.

29. Kwak, H. S., Kin, H., Jae, M. H. and Tae-Ho, S., (2009) Thermal Control of Electroosmotic Flow in a Microchannel through Temperature-dependent Properties, Journal of Colloid and Interface Science, 335 pp. 123-129.

30. Vandoormall, J. P. and Raithby, G. D., (1984) Enhancements of the Simple Method for Predicting Incompressible Fluid Flow, Numerical Heat Transfer, 7 pp. 147-163.

31. Leonard, B. P., (1995) Order of Accuracy of Quick and Related Convection-Diffusion Schemes, Appl. Math. Model., 19 p. 640.

32. Yang, K. S., Chu, W. H., Chen, I. Y. and Wang, C. C., (2007) A Comparative Study of the Airside Performance of Heat Sinks Having Pin Fin Configurations, International Journal of Heat and Mass Transfer, 50 pp. 4661-4667. 
33. Kosar, A., Peles, Y., (2006) Thermal-Hydraulic Performance of MEMS-based Pin-Fin Heat Sink, Journal of Heat Transfer,128 (2) pp. 121-131.

34. Ohmi, K., Coutanceau, M., Loc , T.P., Dulieu, A., (1990) Vortex formation around an oscillating and translating airfoil at large incidences, Journal of Fluid Mechanics, (211), $37-60$.

35. Argade, R., Ghosh, S., De, S., DasGupta, S.,(2007) Experimental investigation of evaporation and condensation in the contact line region of a thin liquid film experiencing small thermal perturbations, Langmuir, (23) 1234-1241.

36. Pratt, D.M., Hallinan, K.P., (1997) An Investigation of Thermocapillary Effects on the Wetting Characteristics of a Heated, Capillary Re-supplied, Curved Meniscus within a Capillary Pore, National Heat Transfer Conference, HTD-vol. 327, vol. 5, pp. 97-104.

37. Chan,S.H., Zhang, W., (1994) Rewetting Theory and the Dryout Heat Flux of Smooth and Grooved Plates with a Uniform Heating, ASME J. Heat Transfer, vol. 116, no. 1, pp. 173-179.

38. Stephan P. C., Busse, C. A.,(1993) Analysis of the Heat Transfer Coefficient of Grooved Heat Pipe EvaporatorWalls, International Journal of Heat and Mass Transfer, vol. 35, no. 2 , pp.383- 391.

39. Ma, H.B., Peterson, G. P., (1997) Temperature Variation and Heat Transfer in Triangular Grooveswith an Evaporating Film, AIAA Journal of Thermophysics and Heat Transfer, vol. 11, no. 1,pp. 90-97, 1997.

40. Ma, H.B., Peterson, G.P., (1998) Disjoining Pressure Effect on the Wetting Characteristics in aCapillary Tube, Microscale Thermophysical Engineering, vol. 2, no. 4, pp. 283-297. 
41. Wayner Jr., P.C., Kao, Y.K., LaCroix, L.V., (1976) The interline heat transfer coefficient ofan evaporating wetting film, Int. J. Heat Mass Transfer (19) 487-492.

42. Wayner Jr., P.C., (1999) Intermolecular forces in phase-change heat transfer :1998Kern award review, AICHE J. 45 (10) 2055-2068.

43. Park, K.,Noh, K.J., Lee, K.S., (2003) Transport phenomenon in the thin-film region of amicro-channel, Int. J. Heat Mass Transfer (46) 2381-2388.

44. Park, K., Lee, K.S., (2003) Flow and heat transfer characteristics of the evaporatingextended meniscus in a micro-capillary channel, Int. J. Heat Mass Transfer (46)4587-4594.

45. Udell, K.S., (1983) Heat transfer in porous media heated from above with evaporation, condensation and capillary effects, Journal of Heat Transfer(105) 485-492.

46. Figus, C., Le Bray, Y., Bories, S., Part, M., (1999) Heat and mass transfer with phase change in a porous structure partially heated: continuum model and pore network simulations, International Journal of Heat and Mass Transfer(42)2557-2569.

47. Cao, Y., Faghri, A., (1994) Conjugate analysis of a flat-plate type evaporator for capillary pumped loops with three-dimensional vapor flow in the groove, International Journal of Heat and Mass Transfer (37)401-409.

48. Cao, Y., Faghri, A., (1994) Analytical solutions of flow and heat transfer in a porous structure with partial heating and evaporation on the upper surface, International Journal of Heat and Mass Transfer(37)1525-1533.

49. Zhao, T.S. Liao, Q., (2000) On Capillary-driven flow and phase-change heat transfer in a porous structure heated by a finned surface: measurements and modeling, International Journal of Heat and Mass Transfer (43)1141-1155. 
50. Liao, Q., Zhao, T.S., (1999) Evaporative heat transfer in a capillary structure heated by a grooved block, Journal of Thermophysics and Heat Transfer (13)126-133.

51. LaClair, T. J.,Mudawar, I., (2000) Thermal transients in a capillary evaporator prior to the initiation of boiling, International Journal of Heat and Mass Transfer (43)3937-3952.

52. Demidov, A.S., Yatsenko, E.S., (1994) Investigation of heat and mass transfer in the evaporation zone of a heat pipe operating by the 'inverted meniscus' principle, International Journal of Heat and Mass Transfer(37)2155-2163.

53. Khrustalev, D., Faghri, A., (1995) Heat transfer in the inverted meniscus type evaporator at high heat fluxes, International Journal of Heat and Mass Transfer(38)3091-3101.

54. Muraoka, I., Ramos, F.M., Vlassov, V.V., (1998) Experimental and theoretical investigation of a capillary pumped loop with a porous element in the condenser, International Communications in Heat and Mass Transfer(25)1085-1094.

55. Muraoka, I., Ramos, F.M., Vlassov, V.V., (2001) Analysis of the operational characteristics and limits of a loop heat pipe with porous element in the condenser, International Journal of Heat and Mass Transfer(44)2287-2297.

56. Kaya, T., Hoang, T.,(1999) Mathematical modeling of loop heat pipes and experimental validation, Journal of Thermo physics and Heat Transfer(13)314-320.

57. Li, H., Liu, Z., Chen, B., Liu, W., Li, C., Yang, J., (2012) Development of biporous wicks for flat-plate loop heat pipe, Experimental Thermal and Fluid Science (37)91-97.

58. Zhang, X., Huo, J., Wang, S., (2012) Experimental investigation on temperature oscillation in a miniature loop heat pipe with flat evaporator, Experimental Thermal and Fluid Science (37)29-36. 
59. Kiseev, V.M., Vlassov, V.V., Muraoka, I., (2010) Experimental optimization of capillary structures for loop heat pipes and heat switches, Applied Thermal Engineering (30)13121319.

60. Berti, L.F., Santos, P.H.D., Bazzo, E., Janssen, R., Hotza, D., Rambo, C.R., (2011)Evaluation of permeability of ceramic wick structures for two phase heat transfer devices, Applied Thermal Engineering (31)1076-1081.

61. Luikov, A. V.,(1975) Systems of differential equations of heat and mass transfer in capillary-porous bodies, International Journal of Heat and Mass Transfer, 18, 1-14.

62. Ogniewicz, Y., Tien, C.L., (1981) Analysis of condensation in porous insulation, International Journal of Heat and Mass Transfer (24)421-429.

63. Tang, Y., Deng, D., Lu, L., Pan,M., Wang, Q., (2010) Experimental investigation on capillary force of composite wick structure by IR thermal imaging camera, Experimental Thermal and Fluid Science (34)190-196.

64. Deng,D., Tang, Y., Huang, G., Lu, L., Yuan, D.,(2013) Characterization of capillary performance of composite wicks for two-phase heat transfer devices, International Journal of Heat and Mass Transfer (56)283-293.

65. Crone, S.,Bergins, C., Strauss, K., (2002) Multiphase flow in homogeneous porous media with phase change. Part I: numerical modeling, Transport in Porous Media (49)291-312.

66. Bergins, C., Crone, S., Strauss, K., (2005)Multiphase flow in porous media with phase change. Part II: analytical solutions and experimental verification for constant pressure steam injection, Transport in Porous Media (60) 275-300. 
67. Brouwers, H.J.H., (1996) An Experimental study of constant-pressure steam injection and transient condensing flow in an air-saturated porous medium, Journal of Heat Transfer (118)449-453.

68. Wyllie, M.R.J., (1962) Relative permeability, chapter 25, Petroleum production handbook, McGraw-Hill, New York 2.

69. Holley, B., Faghri, A.,(2006) Permeability and effective pore radius measurements for heat pipe and fuel cell applications, Applied Thermal Engineering (26) 448-462.

70. Lin, F.C., Liu, B.H., Juan, C.C., Chen, Y.M., (2011) Effect of pore size distribution in bidisperse wick on heat transfer in a loop heat pipe, Heat Mass Transfer (47) 933-940. 


\section{VITA}

Husam finished high school in 2004 with honorable academic and athletic achievements. He joined the University of Missouri-Rolla and was among a group of successful candidates were funded to study bachelors, masters and ph.d in aerospace engineering.

Rajab earned his English diploma in 2006 from Missouri University of Science and Technology. He received his Bachelors (BSc, BSME) and Masters (MI mathematics, MSc) in Mechanical Engineering in 2010 and 2012 respectively. He then started his doctorate studies focusing on nanofluids and phase change materials and their applications.

He worked in various areas including heat sink and heat exchangers, two phase flow, condensation and evaporation, and fabrication and testing of pin fin heat sinks. Husam hopes to receive his $\mathrm{Ph} . \mathrm{D}$ in aerospace and mechanical engineering from the University of MissouriColumbia in the spring of 2017.

Husam has over five years of experience as a design and manufacturing engineer in Industrial and Technological Development Center, ITECH D\&M \& AAF. He joined Center for Nanotechnology and Thermal Management at MU College of Engineering. He has four years of experience in 3D Computer Aided Design, Steam Power, and Fabrication and Testing of Power Plants Systems for Electricity Generation.

Rajab is innovative, motivated and has extensive experience in $3 \mathrm{D}$ plastic \& metal printing, CNC machining, and metal injection molding. He serves as a member of many associations; American Society of Mechanical Engineers, Society of Manufacturing Engineers, and American Society of Thermal \& Fluids Engineers.

Husam participated in the International Mechanical Engineering Congress and Exposition (IMECE) in Montreal, Canada and the Early Career Technical Conference (ECTC) at Georgia Institute of Technology, both as a speaker and workshop presenter. 

EXCHANGER

\author{
MU College of Engineering \\ Candidate for the Degree of Doctor of Philosophy
}

Major Field: Mechanical and Aerospace Engineering

Scope and Method of Study: Vapor condensation condenser consists of two major heat transfer processes, i.e., vapor condensation and forced convection. In order to enhance heat transfer, the condensation heat transfer utilizing the porous medium is investigated, and at the same time, the elliptical pin fin effect on the forced convection of nanofluid studied. The forced convective heat transfer on nanofluids in an elliptical pin-fin heat sink of two different pin orientations is numerically studied by using a finite volume method. With increasing Reynolds number, the recirculation zones behind the pins increases. There are more recirculation zones for the pins with different angular orientations than for pins with the same angular orientation. It is observed that the Nusselt number for the pins with different angular orientations is higher than that for pins with the same angular orientation. With increasing volume fraction of nanoparticles and angular orientation of pins for a given Reynolds number, Euler and Nusselt numbers as well as overall heat transfer efficiency increase.

Findings and Conclusions: In this work, the non dimensional mass, momentum and energy equations based on non dimensional pressure, temperature, heat capacity, capillary, and bond numbers are developed for the vapor condensation occurring in the porous medium. The volumetric viscous force for the flow is described by Darcy's law. For the microscopic interfacial shear stress, a permeability term that relates flow rate and fluid physical properties (i.e. viscosity) to pressure gradient is implemented. The effects of permeability, porosity, and effective pore radius parameters on porous medium performance are introduced. The occurrence of thin liquid film inside pores is addressed and the Laplace-Young equation is depicted. In calculations the Darcy-Ergun momentum relation is implemented which provide accurate means to determine the capillary performance parameters of porous medium. The dimensionless thicknesses of the twophase and liquid regions and embodiment of unique characteristics based on the total thermal diffusivity and absolute permeability are depicted. Therefore, analyses of the phase change and two-phase flow are made by defining regions, over which appropriate approximations are made.

Acknowledgement: The support of the MU Innovations Fund Center (IF) \& the support of the MU College of engineering cnc milling machine center are gratefully acknowledged. 